\title{
Chronic depression : an integrative approach to the study of underlying vulnerability factors and psychological treatment
}

\author{
Citation for published version (APA): \\ Renner, F. (2014). Chronic depression : an integrative approach to the study of underlying vulnerability \\ factors and psychological treatment. [Doctoral Thesis, Maastricht University]. Datawyse / Universitaire \\ Pers Maastricht. https://doi.org/10.26481/dis.20141024fr
}

Document status and date:

Published: 01/01/2014

DOI:

10.26481/dis.20141024fr

Document Version:

Publisher's PDF, also known as Version of record

\section{Please check the document version of this publication:}

- A submitted manuscript is the version of the article upon submission and before peer-review. There can be important differences between the submitted version and the official published version of record. People interested in the research are advised to contact the author for the final version of the publication, or visit the DOI to the publisher's website.

- The final author version and the galley proof are versions of the publication after peer review.

- The final published version features the final layout of the paper including the volume, issue and page numbers.

Link to publication

\footnotetext{
General rights rights.

- You may freely distribute the URL identifying the publication in the public portal. please follow below link for the End User Agreement:

www.umlib.nl/taverne-license

Take down policy

If you believe that this document breaches copyright please contact us at:

repository@maastrichtuniversity.nl

providing details and we will investigate your claim.
}

Copyright and moral rights for the publications made accessible in the public portal are retained by the authors and/or other copyright owners and it is a condition of accessing publications that users recognise and abide by the legal requirements associated with these

- Users may download and print one copy of any publication from the public portal for the purpose of private study or research.

- You may not further distribute the material or use it for any profit-making activity or commercial gain

If the publication is distributed under the terms of Article $25 \mathrm{fa}$ of the Dutch Copyright Act, indicated by the "Taverne" license above, 
ด Q ด

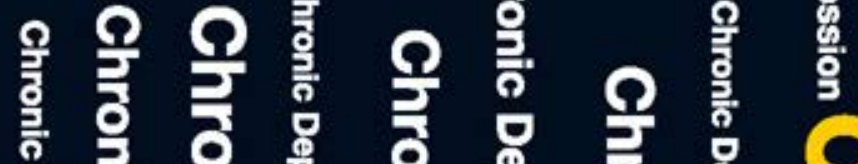

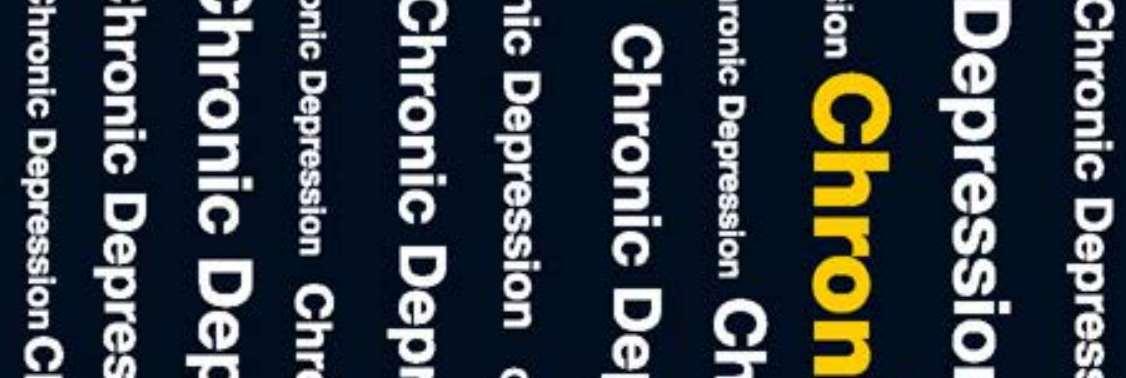

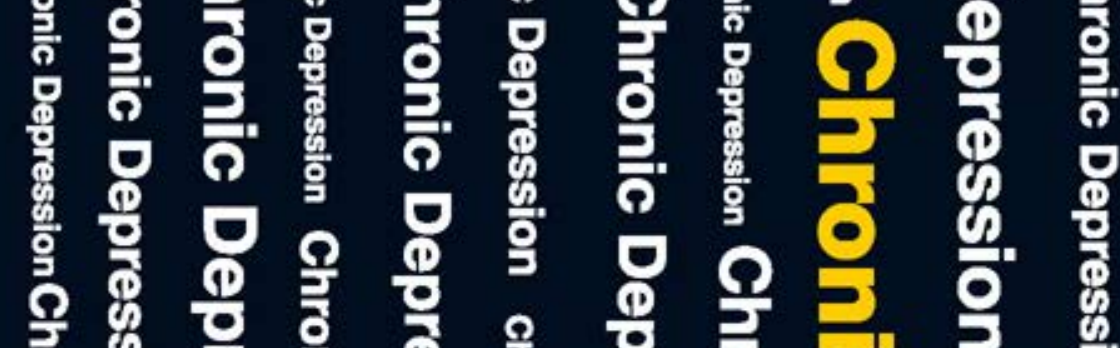

产 参 웅

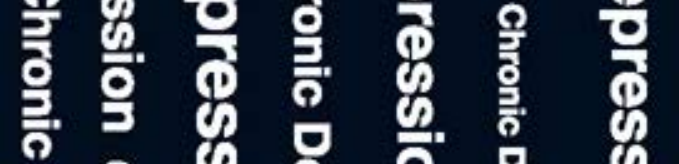

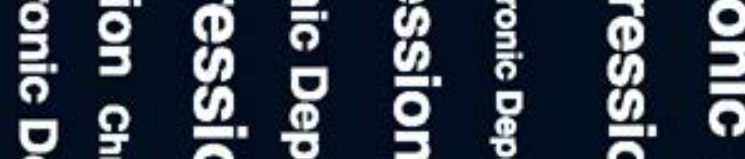

。ㅇㅎㅇ

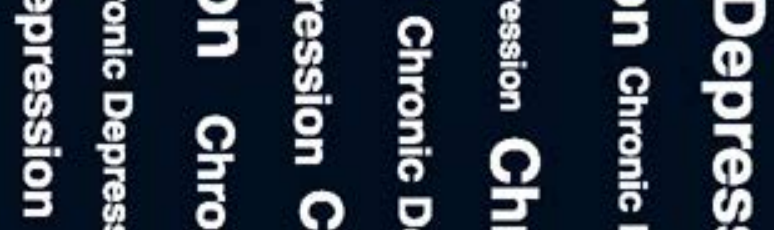

응 응 용

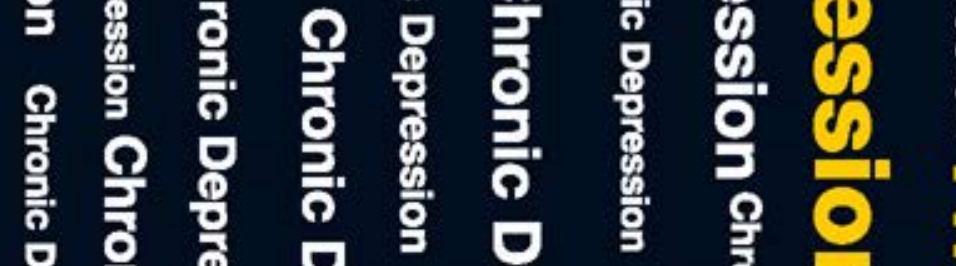

它产

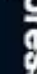

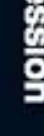

응

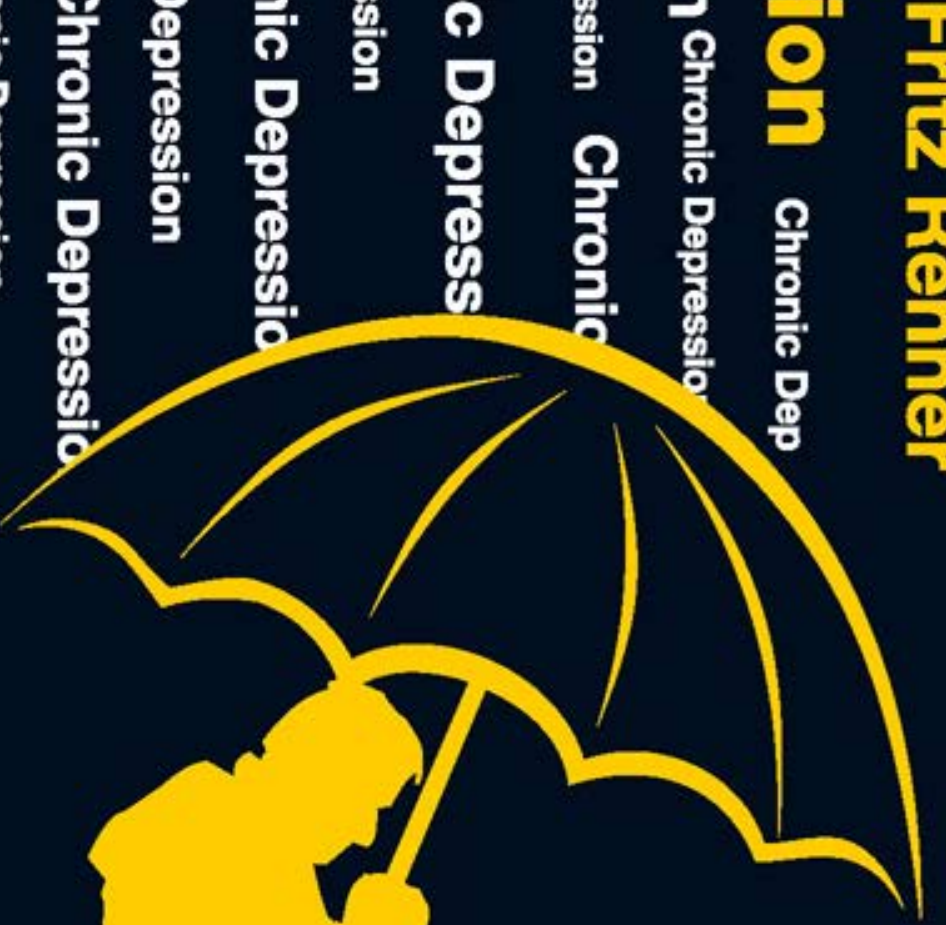

용

용 올 올 올

要

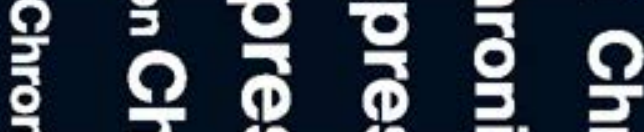

द \& के

음 둥 웅

융 응 웅 क्षे

○

.

है क

() है

व

$\Rightarrow$ म

ज क क क

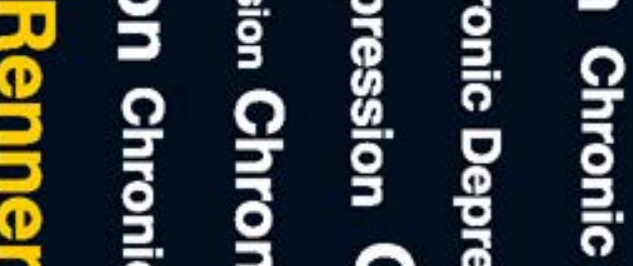

คำ ค \&

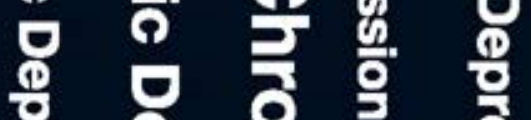

- 1105

8 ? 0 ?

क क

g

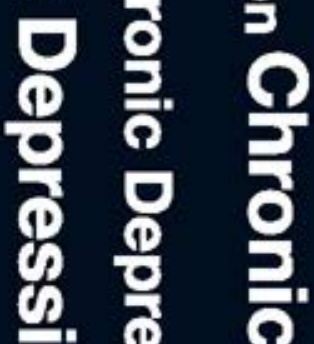

An integrative approach

용

to the study of underlying

vulnerability factors and

6. 0

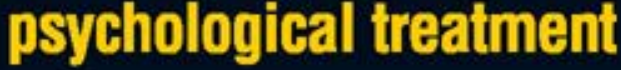

- 10 
(C) Fritz Renner, Maastricht 2014

\section{ISBN 9789461593696}

Production: Datawyse | Universitaire Pers Maastricht Cover design: Desry Turnip 


\title{
Chronic Depression
}

\section{An integrative approach to the study of underlying vulnerability factors and psychological treatment}

\author{
Proefschrift \\ ter verkrijging van de graad van doctor aan de Universiteit Maastricht, \\ op gezag van de Rector Magnificus, Prof. dr. L.L.G. Soete, \\ volgens het besluit van het College van Decanen, in het openbaar te verdedigen \\ op vrijdag 24 oktober 2014 om 16.00 uur door
}

Fritz Renner

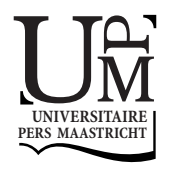




\section{Promotoren}

Prof. dr. M.J.H. Huibers (Vrije Universiteit Amsterdam)

Prof. dr. A. Arntz (Universiteit van Amsterdam)

\section{Copromotor}

Prof. dr. F.P.M.L. Peeters

\section{Beoordelingscommissie}

Prof. dr. P. Muris (voorzitter)

Prof. dr. D.P. Bernstein

Prof. dr. C.L.H. Bockting (Rijksuniversiteit Groningen)

Dr. J. Roelofs

Prof. dr. J. Spijker (Radboud Universiteit Nijmegen) 


\section{Contents}

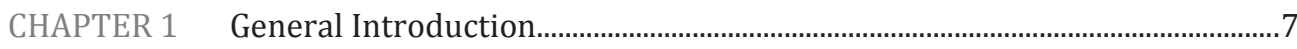

PART I NEUROIMAGING STUDIES IN CHRONIC DEPRESSION ....... 25

CHAPTER 2 Neural correlates of self-referential processing in chronic

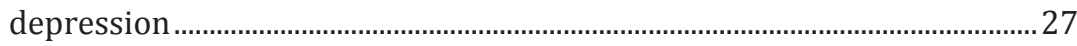

CHAPTER 3 Sad mood induction modulates default mode network connectivity in chronic depression ......................................................................................... 51

PART II STABILITY OF UNDERLYING RISK-FACTORS FOR

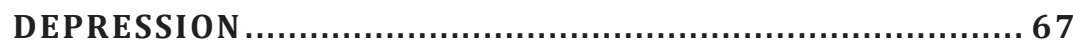

CHAPTER 4 Stability of schemas during treatment for depression ................................69

CHAPTER 5 Stability of personality traits in treated and untreated patients with

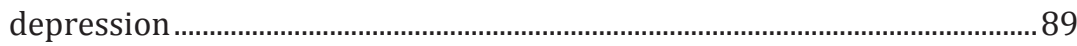

CHAPTER 6 Stability of interpersonal problems during cognitive therapy for

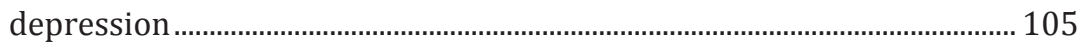

CHAPTER 7 Effects of psychotherapy for depression on social functioning ................ 125

PART III SCHEMA THERAPY FOR CHRONIC DEPRESSION ............ 149

CHAPTER 8 Schema therapy for chronic depression: Rationale ..................................... 151

CHAPTER 9 Effects of schema therapy for chronic depression ..................................... 173

CHAPTER 10 Exploring mechanisms of change in schema therapy for chronic

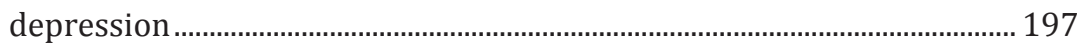

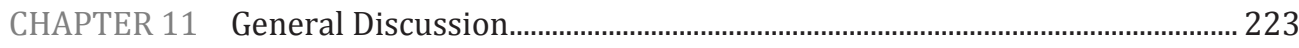

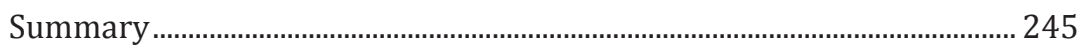

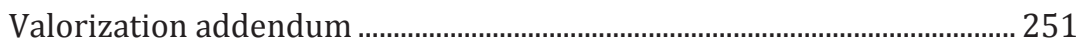

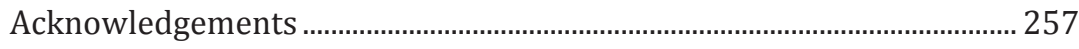

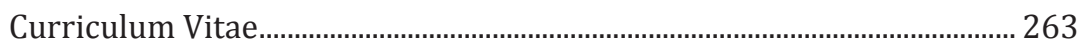





\section{CHAPTER 1 \\ General Introduction}


Feeling depressed, down or blue... People use various words to describe negative mood states and probably everyone has experienced moments of sad mood in their lives. For most people sad mood comes and goes but for some people depressed mood persists over several days, weeks, months or years. But depression is more than feeling sad: People with depression often have lost interest or pleasure in almost all activities; they feel worthless and guilty; have trouble concentrating and taking decisions and often have sleeping problems. These and other symptoms of depression cause many problems in everyday live, such that people with depression have difficulties in engaging in social activities, functioning at work and maintaining healthy relationships. In the worst case depression can lead to suicide. Due to these negative consequences, depression is one of the leading causes of disability worldwide.

This dissertation describes a number of studies that my co-authors and I have conducted on underlying neurological and psychological vulnerability factors in (chronic) depression and on the stability of psychological vulnerability factors during treatment for depression. The theoretical application of a new form of psychotherapy, schema therapy, for patients with chronic depression is presented as well as the results of a single-case series testing the effects of schema therapy for patients with chronic major depressive disorder. Finally, the results of a study on potential mechanisms of change in schema therapy for chronic depression are presented. The introduction to this dissertation provides a general overview on the classification of major depressive disorder and chronic forms of depression, a brief review on the prevalence and negative consequences of chronic depression, an overview of current treatment options for chronic depression, a summary on the underlying developmental, psychological and neurological disturbances underlying chronic depression, and a short overview on the key concepts of schema therapy as well as a review of the current evidence base of schema therapy. At the end of the introduction the aims and outline of this dissertation are summarized.

\section{Major Depressive Disorder}

Major depressive disorder is a common mood disorder characterized by low mood, a loss of interest and a number of other emotional, cognitive and physical disturbances such as feelings of worthlessness or a loss of appetite (American Psychiatric Association, 2013). Table 1 provides an overview of the full Diagnostic and Statistical Manual of Mental Disorders fifth edition (DSM-V) diagnostic criteria of major depressive disorder. The criteria of a major depressive episode are met if five or more of these criteria are present, including either the depressed mood or the loss of interest criteria, during a period of two weeks nearly every day most of the day.

Recent epidemiological studies estimate that the lifetime prevalence for a major depressive episode varies between $11.1 \%$ in low income countries and $14.6 \%$ in high income countries (Bromet et al., 2011). Since 1990 depression moved from place four of the leading causes of disease burden worldwide to place two, highlighting that depression is a major global health problem (Ferrari et al., 2013). Findings from the 
2010 global burden of disease study have shown that among mental disorders, depression, including dysthymia, account for the greatest burden of disease worldwide (Whiteford et al., 2013), emphasizing the importance of effective treatments for depression.

Table 1. DSM-5 diagnostic criteria of Major Depressive Disorder (see print version).

Among others, common treatment options for major depressive disorder include antidepressant medication, psychotherapy, or the combination of antidepressant medication and psychotherapy. A recent meta-analysis found that $62 \%$ of patients with depression do not meet the criteria for depression anymore after psychotherapy (Cuijpers et al., 2014) and comparative outcome studies between different types of psychotherapy suggest that there are no differences between different types of psychotherapy in treatment effects (Cuijpers, van Straten, Andersson, \& van Oppen, 2008). Comparative outcome studies between psychotherapy and antidepressants suggest that antidepressants and psychotherapy are approximately equally effective in reducing depressive symptoms following treatment (for a meta-analysis see: Cuijpers et al., 2013) but psychotherapy seems to have more positive long-term effects than antidepressants (for a meta-analysis see: Imel, Malterer, McKay, \& Wampold, 2008). Combined treatments are slightly more effective than either intervention alone (for meta-analyses see: Cuijpers, Dekker, Hollon, \& Andersson, 2009; Cuijpers, van Straten, Warmerdam, \& Andersson, 2009; Khan, Faucett, Lichtenberg, Kirsch, \& Brown, 2012). Although the majority of patients improve during treatment some patients with depression do not get better in treatment. If symptoms of depression do not improve, either with treatment or without treatment, depression can become a chronic condition (for a review see: Richards, 2011). 


\section{A brief history of the classification of chronic depression}

The concept of chronic depression as an axis-I disorder was first introduced in the third edition of the DSM classification system (American Psychiatric Association, 1980). Before that, long lasting forms of depression were seen as characterological disorders (for a historical review see: Akiskal, 2001). In general, a depression is considered to be chronic when the symptoms of depression are present for two years or longer. The DSMIII included a separate diagnostic category for patients with long lasting depressive symptoms that did not meet all criteria of a depressive disorder, i.e. dysthymic disorder. In addition to dysthymic disorder as a separate diagnostic category, the revised DSM-III and the DSM-IV included specifiers for a more severe form of chronic depression (chronic major depressive disorder) (American Psychiatric Association, 1987, 2005). In addition, a recurrent major depressive disorder without full interepisode recovery and a major depressive episode superimposed on dysthymic disorder (double depression; Keller \& Shapiro, 1982) are considered chronic forms of depression and are represented in the DSM-IV by course specifiers. Figure 1 provides a graphical overview of these four different types of chronic depression.
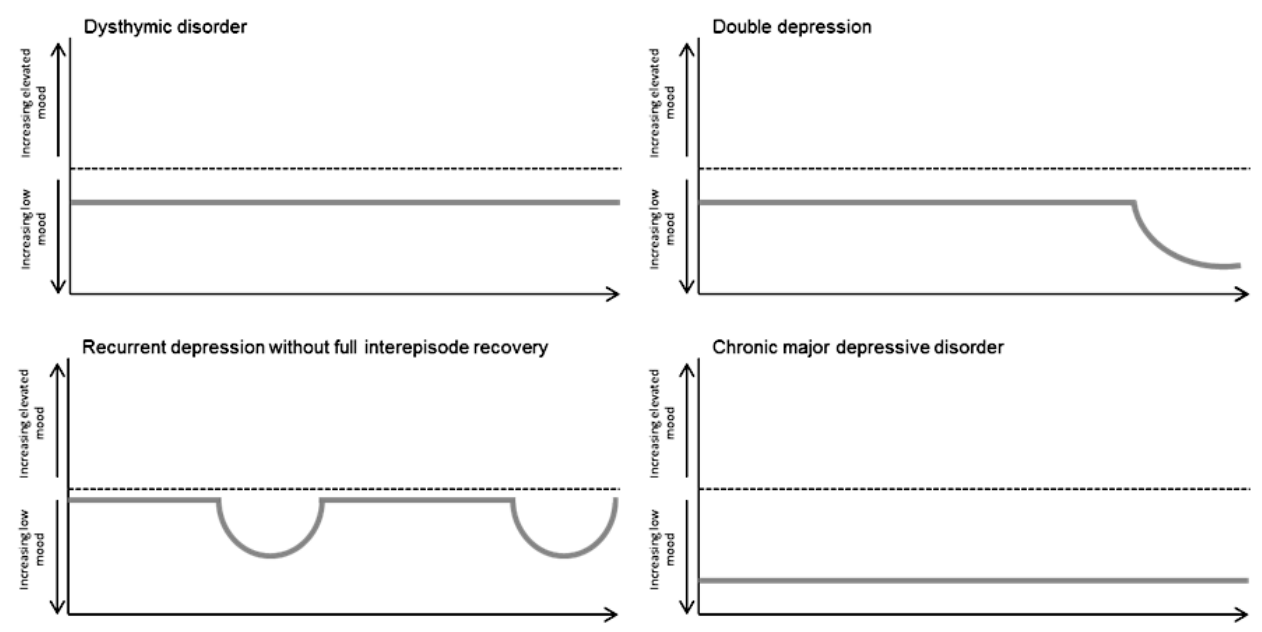

Figure 1. Graphical representation of different types of chronic depression. The dashed line represents an euthymic state (i.e. normal mood). Depressed mood is indicated by the area under the dashed line whereas elevated mood is indicated by the area above the dashed line. The $x$-axis represents passing of time over 2 years. 
These different forms of chronic depression appear not to differ from each other with respect to a number of demographic, clinical and psychosocial variables and it has therefore been argued that chronic depression should be conceptualized as a single disorder (McCullough et al., 2003; McCullough et al., 2000). In line with this, in the most recent DSM-5 classification, the different subtypes of chronic depression have been consolidated into one single category: persistent depressive disorder. The criteria for a persistent depressive disorder are met if a person experiences depressed mood most of the day nearly every day for two years in addition to at least two out of six of the following criteria: poor appetite or overeating; insomnia or hypersomnia; low energy or fatigue; low self-esteem; poor concentration or difficulty making decisions; feelings of hopelessness (American Psychiatric Association, 2013). All research that is cited and all research that was conducted in this dissertation is based on previous diagnostic classification systems and therefore, throughout this dissertation, the more common term chronic depression will be used to refer to a depression that is present for two years or longer.

\section{Prevalence and consequences of chronic depression}

A relatively large proportion of patients with depression develop a chronic course: Findings from the Sequenced Treatment Alternatives to Relieve Depression trial (Rush et al., 2004) have shown that among 2876 patients with major depressive disorder $25.3 \%$ had a current episode that lasted 2 years or longer (Trivedi et al., 2006). Comparable prevalence rates of chronic depression among patients with major depression have been reported in epidemiological studies: The Netherlands Mental Health Survey and Incidence Study found that $20 \%$ of the patients with a major depressive episode had not recovered within two years (Spijker et al., 2002).

Given that, by definition, chronic forms of depression are characterized by longer depressive episodes, it is reasonable to assume that chronic forms of depression contribute more to the burden of disease than non-chronic forms of depression. In line with this argument, it has been shown previously, that economic costs of depression are explained by the duration of symptoms, rather than by the severity of symptoms, indicating that chronic forms of depression are associated with higher economic burden (Fostick, Silberman, Beckman, Spivak, \& Amital, 2010). A number of studies have compared the negative consequences of depression in patients with and without chronic depression. Compared to episodic forms of depression, chronic depression is characterized by increased impairments in psychosocial functioning (e.g. Wells, Burnam, Rogers, \& Hays, 1992), is associated with more suicide attempts and hospitalizations, higher service use and increased economic costs (for a review see: Arnow \& Constantino, 2003). Findings from an epidemiological survey study (Netherlands Mental Health Survey and Incidence Study) have shown that people with chronic forms of depression have lower recovery rates and are more likely to have any depressive disorder after three year follow-up compared to people with an non-chronic depression (Rhebergen et al., 2009). In the light of the relatively high prevalence of chronic depression and the 
negative consequences of chronic depression it is critical to obtain a better understanding of the underlying vulnerability factors of chronic depression and to explore new treatment options.

\section{Current treatment of chronic depression}

When discussing the effects of treatments for depression it is crucial to distinguish between different terms that can be used to describe treatment effects. Table 2 provides common definitions of terms that are often used in depression treatment outcome studies and meta-analyses to describe treatment effects. One useful conceptualization in this context has been the introduction of the terms "response" "remission" and "recovery" to describe treatment effects (Frank et al., 1991). Moreover, in meta-analyses treatment effects are often described in terms of effect sizes representing standardized improvement on depression severity scales. Given that patients who have reached a response or only partial remission are usually still symptomatic and therefore at an increased risk of relapse (Paykel, 2008), it has been argued that full remission should be the desired treatment outcome in depression (Thase, 2003).

Table 2. Terms commonly used to define treatment effects in treatment outcome studies or metaanalyses
Term
Definition
Response A percentage change from the initial baseline score (often about 50\%) on a depression symptom severity measure (Riedel et al., 2010).
Remission Reaching an absolute cutoff score (usually corresponding to a minimum or total absence of symptoms) on a depression symptom severity measure (Riedel et al., 2010) or diagnostic interview. In the DSM 5 classification system, partial remission refers to a state in which some symptoms of depression are present but the criteria for a depressive episode are not met or a state in which no symptoms of depression are present for less than two months. Full remission refers to a state in which no significant symptoms of depression are present for a period of two months or longer (American Psychiatric Association, 2013).

Recovery Not meeting diagnostic criteria for depression on a diagnostic interview.

Effect sizes Standardized improvement on depression severity scales.

Currents treatments for chronic depression include psychotherapy, antidepressant medication or the combination of antidepressant medication and psychotherapy. Psychotherapy has been shown to be effective in reducing depressive symptoms in patients with chronic depression but the effect sizes are small (for a meta analysis see: Cuijpers et al., 2010). More promising results have been reported for the cognitive behavioral analysis system of psychotherapy (CBASP), a relatively new type of psychotherapy that was specifically developed for chronic depression. In a large randomized controlled trial comparing the efficacy of CBASP, antidepressants and their combination for patients with chronic depression, it has been shown that up to $48 \%$ of the patients randomized to CBASP showed a response and $33 \%$ of the patients in the 
CBASP condition reached remission (Keller et al., 2000). In the same trial, remission rates following antidepressant medication was 29\% (Keller et al., 2000). The most positive results have been reported for the combination of psychotherapy and medications (for a review see: Pampallona, Bollini, Tibaldi, Kupelnick, \& Munizza, 2004; Spijker, van Straten, Bockting, Meeuwissen, \& van Balkom, 2013). In the previously mentioned CBASP trial it has been shown, for example, that up to $73 \%$ of patients with chronic depression responded to combined treatment (48\% remitted), compared to a $48 \%$ response rate for patients receiving either antidepressants or psychotherapy alone (Keller et al., 2000).

Although such findings show that current treatments for chronic depression can be effective, about half of the patients do not reach remission in treatment, even with combined treatments, and are therefore at an elevated risk of subsequent relapses and recurrences, illustrating that there is still a lot of room for improvement. Another problem with current treatment approaches for chronic depression is that the effects are often not enduring. Even if patients with chronic depression reach full remission in treatment they might still be at an elevated risk for relapse: Findings from the Sequenced Treatment Alternatives to Relieve Depression study have shown that relapse among patients who have reached remission was more likely in those with a prior chronic course of depression (Rush et al., 2012). Such findings indicate that extensive continuation and maintenance treatment for chronic depression is required in order to prevent relapses and recurrences. It has been shown, for example, that patients with chronic depression who initially responded to treatment experience less recurrence one year after treatment if they continued medication compared to pill placebo (Gelenberg et al., 2003).

Taken together, while current treatment options for chronic depression are effective, these effects are rather small and often not maintained when treatment is terminated. Moreover, even if patients with chronic depression reach remission they are still at an elevated risk for relapse. Therefore, it is important to take a closer look at the underlying vulnerability factors that might maintain chronic depression when exploring novel treatment options in order to get a better understanding of the requirements of potentially effective treatments.

\section{Developmental and psychological vulnerability factors underlying chronic depression}

Are there developmental and psychological factors that distinguish episodic forms of depression from chronic depression? In recent years, a number of studies have been conducted to determine the underlying risk-factors of chronic depression. One developmental risk-factor that has often been associated with chronic depression is childhood abuse (Brown, Craig, Harris, Handley, \& Harvey, 2007; Wiersma et al., 2009). Childhood abuse has been shown to moderate treatment response in chronic depression: Patients with chronic depression and a history of childhood abuse are less responsive to antidepressants, compared to patients with chronic depression and no history of 
childhood abuse (Klein et al., 2009). Furthermore, another study found that patients with chronic depression and a history of childhood trauma benefit more from psychotherapy than pharmacotherapy (Nemeroff et al., 2003).

Chronic depression has also been associated with dysfunctional cognitive factors. It has been shown, for example, that patients with chronic depression score higher on measures of dysfunctional cognitions compared to non-chronically depressed patients (Riso et al., 2003).

Patients with chronic depression also have an increased comorbidity of axis-I and axis-II disorders (for a review see: Riso, Miyatake, \& Thase, 2002). There is mixed evidence as to whether co-morbid axis-II disorders have a negative impact on treatment outcomes in depression. While some studies found that axis-II comorbidity in patients with depression has a negative impact on treatment outcome (e.g. Newton-Howes, Tyrer, \& Johnson, 2006; Newton-Howes et al., 2013), other studies did not find evidence for this association (e.g. Maddux et al., 2009).

Finally, it has been shown that interpersonal problems predict a chronic course of depression (Brown \& Moran, 1994) and that patients with chronic depression show increased social avoidance compared to patients with episodic depression (Ley et al., 2011).

A more detailed description of the underlying developmental and psychological risk-factors to chronic depression is provided in chapter 8.

\section{Neurological vulnerability factors underlying depression}

In addition to the developmental and psychological risk-factors, it is important to also identify specific brain regions and neural networks that are associated with abnormal cognitive processing in chronic depression. Towards this end a number of structural and functional neuroimaging studies, primarily in patients with episodic major depressive disorder, have been conducted in recent years. These studies have made use of modern neuroimaging techniques such as Magnetic Resonance Imaging (MRI) or functional (f)MRI to measure differences in brain structures between depressed and nondepressed individuals as well as metabolic changes as an index of neural activity in these brain structures, respectively. (f)MRI is a non-invasive technique that makes use of strong magnetic fields to measure brain tissue and the amount of deoxygenated hemoglobin in the blood as an indicator of brain activity. Most functional neuroimaging studies in depression have focused on cognitive and emotional functions that are distorted in depression such as processing of emotional stimuli, reward seeking and emotion regulation (for a review see: Kupfer, Frank, \& Phillips, 2012). Studies that have focused on disturbed emotional processing have shown that compared to healthy controls, patients with depression show increased neural activity in subcortical systems (e.g. amygdala, insula) and lower responses in prefrontal cortical areas (e.g. dorsolateral prefrontal cortex) during processing of negative information (for a meta-analysis see: Hamilton et al., 2012). Other studies have focused on identifying the neural correlates of distorted cognitive processing in depression, such as an increased processing of 
negative information with regard to the self (i.e. self-referential processing; Northoff, 2007). Studies in this area have emphasized the role of cortical midline structures, including the medial prefrontal cortex, anterior cingulate cortex, as well as the posterior medial cortices during self-referential processing in patients with depression (for reviews see: Lemogne, Delaveau, Freton, Guionnet, \& Fossati, 2012; Nejad, Fossati, \& Lemogne, 2013).

In most functional neuroimaging studies participants are typically presented with emotional stimuli such as fearful faces or emotional words and have to engage in a cognitive task as part of the experimental paradigm that is used. Another line of research has focused on so called resting state networks to determine the connectivity within distinct brain networks in depression (Wang, Hermens, Hickie, \& Lagopoulos, 2012). In these studies participants are typically instructed to rest and relax while no further task instructions are provided. Resting state studies have identified a set of distinct neural networks in patients with depression as well as in other mental disorders (for a review see: Broyd et al., 2009). Although these studies have led to an increased understanding of the underlying neurological dysfunctions in depression, most studies in this area were performed in patients with episodic forms of depression and it therefore remains unclear if the findings in patients with episodic depression generalize to those with chronic depression.

Given that patients with chronic depression by definition suffer more long-lasting depressive episodes, it is possible that they are also characterized by differential neurological processing during task-performance as well as during rest. Greicius and colleagues (2007), for example, identified a depressed neural network and found that connectivity within this network varied as a function of the length of the current depressive episode (i.e. chronicity). In the current dissertation the functional connectivity within depressogenic brain networks during rest in patients with chronic depression will further be investigated in chapter 3.

\section{Implications of underlying vulnerability factors for treatment of chronic depression}

As has been summarized in the previous paragraphs, studies on the underlying riskfactors of chronic depression have shown that chronic depression is characterized by a broad range of underlying psychological and developmental risk-factors. In addition, a number of abnormalities in neural processing during performance in cognitive tasks as well as distinct brain networks during rest have been identified but most studies in this area have been conducted in patients with episodic depression. It is therefore important to further study underlining neurological, developmental and psychological risk-factors to chronic depression. The identification of developmental and psychological riskfactors has important implications for the treatment of chronic depression. Treatment for chronic depression should take the developmental origins of depression into account (adverse childhood events), current problems that might maintain the depression (interpersonal factors and dysfunctional cognitions) as well as possible personality 
pathology that might interfere with the psychotherapeutic process. Given the wide range of underlying risk-factors, it seems unlikely that any form of psychotherapy with a primary focus on only one of these aspects can achieve positive and enduring effects in chronic depression. Instead, more positive results might be achieved with an integrative treatment approach to chronic depression that takes all of the underlying risk-factors into account.

\section{Schema Therapy - an integrative treatment approach to chronic depression}

Schema therapy is such an integrative treatment approach combining cognitivebehavioral, interpersonal, experiential and psychodynamic techniques (Young, Klosko, \& Weishaar, 2003). Young et al. developed schema therapy as a novel treatment for complex and difficult to treat patients, particularly those with chronic depression and personality pathology (Young et al., 2003). The underlying model of schema therapy assumes that patients with psychological problems are characterized by a distinct set of negative core beliefs, so called early maladaptive schemas (EMS). EMS determine the way in which people perceive the world, themselves and others and can have a powerful impact on sensations, emotions and behavior. According to the schema model, EMS are formed early in life and remain stable throughout life if no attempts are made to change them. Individuals who experience negative traumatic events early during childhood might form a distinct set of strong negative EMS. Later in life these EMS might be activated by current stressors or environmental events. For example, a student with a strong failure schema might activate the schema in situations in which academic performance is assessed. Activation of the failure schema might then go along with negative emotions and dysfunctional thoughts about the self which contributes to depression.

Another key concept in schema theory are schema modes. Schema modes describe a set of concurrently activated EMS and coping responses (Young et al., 2003). Unlike EMS, schema modes describe the moment-to-moment emotional states of a patient. Different psychological disorders are characterized by different set of predominant schema modes and patients might switch between different sets of schema modes. While a number of studies have assessed EMS in patients with depression (e.g., Riso et al., 2006) there is a lack of research on schema modes in depression. In chapter 8 hypothetical examples of schema modes in chronically depressed patients are discussed and it is explained how therapists can deal with schema modes in schema therapy.

\section{Schema Therapy - Evidence Base}

In recent years a number of treatment studies have been conducted to test the effects of schema therapy for various axis-I and axis-II disorders (for a review see: Bamelis, Bloo, Bernstein, \& Arntz, 2012). Schema therapy has been shown to be an effective treatment for patients with borderline personality disorder (Dickhaut \& Arntz, 2014; Farrell, Shaw, \& Webber, 2009; Giesen-Bloo et al., 2006; Nadort et al., 2009; Nordahl \& Nysaeter, 2005) 
and cluster-c paranoid, histrionic and narcissistic personality disorders (Bamelis, Evers, Spinhoven, \& Arntz, 2013). Moreover, schema therapy in a group setting, combined with treatment as usual, has been shown to be more effective than treatment as usual alone for patients with borderline personality disorder (Farrell et al., 2009). Finally, a shortened version of group schema therapy with a stronger focus on cognitive techniques and EMS (van Vreeswijk \& Broersen, 2006) has been shown to have positive effects on general symptomatic distress in mixed clinical groups (van Vreeswijk, Spinhoven, Eurelings-Bontekoe, \& Broersen, 2012) and in young adults with personality disorders and personality disorder features (Renner et al., 2013). Given that patients with chronic depression often report a history of childhood abuse and co-morbid axis-II disorders, schema therapy might be a valuable treatment option for these patients.

Only a few studies to date have tested the effects of schema therapy in patients with (chronic) depression. In a randomized clinical trial comparing schema therapy, client centered therapy and treatment as usual for patients with predominantly cluster-c personality disorders the effects of these treatments on co-morbid depression have been investigated as secondary outcomes (Bamelis et al., 2013). The authors found that patients randomized to schema therapy had a lower risk of meeting the diagnostic criteria for a depressive disorder at the end of the three-year follow up phase compared to patients who were randomized to the treatment as usual condition (Bamelis et al., 2013). Brewin et al. (2009) found large treatment effects of imagery rescripting, a core technique in schema therapy, for treating patients with chronic depression. A recent randomized clinical trial examined the effects of a relatively short protocol of schema therapy vs. cognitive behavioral therapy for patients with (chronic) depression (Carter et al., 2013). The authors found that at the end of the 12 months study period $50 \%$ of the patients in the schema therapy condition and $40 \%$ of patients in the cognitive therapy condition were in remission. These remission rates did not differ statistically significantly, indicating that schema therapy is at least as effective as cognitive therapy for patients with depression (Carter et al., 2013), though it should be noted that the study was probably underpowered and was terminated before the planned sample sizes were reached. Chronicity did not differentially impact treatment outcome, suggesting that a relatively short protocol of schema therapy is suitable for patients with episodic as well as for patients with chronic depression (Carter et al., 2013). Finally, a single-case series of schema therapy in 12 patients with chronic depression found large effects of schema therapy on improvements in depressive symptoms and approximately $60 \%$ remission rates (Malogiannis et al., 2014). Although these initial findings are promising, there is a need to further investigate the effects of schema therapy for patients with chronic depression: The average number of weekly sessions in the schema therapy condition in the study by Carter et al. (2013) was relatively small (18 weekly sessions). Given that the effects of psychotherapy for chronic depression increase with the number of therapy sessions (Cuijpers et al., 2010), it is important to investigate the effects of a more intensive course of schema therapy for patients with chronic depression. 


\section{Aims of the current dissertation}

Chronic depression is a complex disorder and current treatments fall short, possibly because the underlying developmental and psychological vulnerability remains untouched. Therefore, there is a need to further investigate the underlying vulnerability to (chronic) depression, the stability of vulnerability factors during treatment and to explore novel treatment options for patients with chronic depression. In recent years there has been an increased interest in schema therapy as a novel treatment option for patients with difficult to treat psychiatric disorders. Most studies so far have focused on the effects of schema therapy in patients with personality disorders. More recently studies emerged that investigated schema therapy-related concepts in patients with depression and initial studies have emerged testing the effects of schema therapy for chronic depression. This dissertation builds upon this work and aims to further extend the knowledge of underlying risk-factors in (chronic) depression and to explore new treatment options for chronic depression.

The primary aim of this dissertation was to test the effects of schema therapy for chronic depression in a single-case series and to explore underlying mechanisms of change in schema therapy for chronic depression. The secondary aim was to examine underlying neurological and psychological vulnerability factors to (chronic) depression, as well as the stability of psychological vulnerability factors throughout treatment. Towards this end, my co-authors and I have conducted a number of studies in patients with episodic forms of depression and in patients with chronic depression. The results of these studies are presented in this dissertation in three parts:

Part I of this dissertation describes the results of two neuroimaging studies in patients with chronic major depressive disorder and healthy controls. The overall aim of part I was to explore the underlying neuropathology of chronic major depressive disorder using fMRI. Specifically, in chapter 2 the neural correlates of self-referential processing and its associations with explicit and implicit measures of self-associations were explored in patients with chronic major depressive disorder and non-patient controls. It was hypothesized that patients with chronic depression would show a differential activation pattern in areas of the brain that have previously been associated with self-referential processing during the presentation of schema-congruent (negative and depression-related negative trait words) vs. schema incongruent (neutral or positive trait words) compared to non-patient controls. The study described in chapter 3 is a neuroimaging study, conducted in the same sample as chapter 2, comparing resting state functional connectivity of the default mode network of patients with chronic major depressive disorder and healthy controls before and after a sad mood induction. It was hypothesized that patients with chronic depression would show increased default mode network connectivity following a sad mood challenge.

Part II of this dissertation focusses on the stability of underlying vulnerability factors of depression during treatment for depression. The overall aim of part II was to test if putative underlying vulnerability factors of depression remain stable or improve during treatment for depression. More specifically, the study described in chapter 4 
explored the relation between early maladaptive schemas and depressive symptoms and the stability of schemas over a course of outpatient treatment for depression in a naturalistic treatment setting. It was hypothesized that schemas describing core themes of depression would be related to depressive symptoms and that schemas would remain stable over the course of treatment. The aim of chapter $\mathbf{5}$ was to explore the stability of the personality traits extraversion and neuroticism in patients with depression who sought treatment and in patients with depression who did not seek treatment during a two-year period. In the study described in chapter 6 the stability of interpersonal problems in patients with depression during a course of cognitive therapy was tested. It was hypothesized that patients would experience less interpersonal distress following cognitive therapy for depression but that the interpersonal style of patients would remain stable. The main question that was addressed in chapter 7 was whether psychotherapy for depression has positive effects on psychosocial functioning. To explore this question, a meta-analysis of randomized clinical trials testing the effects of psychotherapy on psychosocial adjustments was conducted. It was hypothesized that psychotherapy for depression would have positive effects on psychosocial functioning and that these effects would be positively correlated with the effects of psychotherapy on depressive symptom severity.

Part III of this dissertation focusses on the application of schema therapy as a novel treatment option for patients with chronic depression. The overall aim of part III was to describe the rationale of applying schema therapy to chronic depression, to test if schema therapy is a potentially effective treatment for chronic depression and to explore underlying mechanisms of change in schema therapy for chronic depression. More specifically, in chapter $\mathbf{8}$ the theoretical application and the underlying rationale of applying schema therapy to chronic depression is described and a general treatment protocol of schema therapy for chronic depression is provided. In the study described in chapter 9, the results of a single case-series study testing the effects of schema therapy for chronic major depressive disorder are described. It was hypothesized that, compared to a non-treatment baseline phase, schema therapy would result in improvements in depressive symptoms, global symptomatic distress and improvements in overall functioning. In the study described in chapter $\mathbf{1 0}$ the temporal relation between depressive symptoms and two potential underlying mechanisms of change in schema therapy for chronic depression (the therapeutic alliance and negative core beliefs) were explored. The main hypotheses were that change in negative core-beliefs would predict subsequent change in depressive symptoms and that session to session change in depressive symptoms would be positively related to the therapeutic alliance rating of the session.

In chapter 11 the results of the current dissertation are integrated and discussed. Methodological shortcomings and recommendations for future studies are provided. In the valorization addendum the societal relevance, target groups and the innovative aspects of this dissertation are described. 


\section{References}

Akiskal, H. S. (2001). Dysthymia and cyclothymia in psychiatric practice a century after Kraepelin. Journal of Affective Disorders, 62(1-2), 17-31.

American Psychiatric Association. (1980). Diagnostic and statistical manual of mental disorders (3rd ed). Washington, DC: American Psychiatric Association.

American Psychiatric Association. (1987). Diagnostic and Statistical Manual of Mental Disorders. Revised. Washington DC: American Psychological Association.

American Psychiatric Association. (2005). Diagnostic and Statistical Manual of Mental Disorders Fourth edition, text revision. Washington, DC: American Psychiatric Association.

American Psychiatric Association. (2013). Diagnostic and statistical manual of mental disorders (5th ed.). Arlington, VA: American Psychiatric Publishing.

Arnow, B. A., \& Constantino, M. J. (2003). Effectiveness of psychotherapy and combination treatment for chronic depression. Journal of Clinical Psychology, 59(8), 893-905. doi: 10.1002/jclp.10181

Bamelis, L. L. M., Bloo, J., Bernstein, D., \& Arntz, A. (2012). Effectivness studies. In M. F. Van Vreeswijk, J. Broersen \& M. Nadort (Eds.), The Wiley-Blackwell handbook of schema therapy: theory, research and practice (pp. 495-510). Chichester: Wiley-Blackwell.

Bamelis, L. L. M., Evers, S. M. A. A., Spinhoven, P., \& Arntz, A. (2013). Results of a multicentered randomized controlled trial on the clinical effectiveness of schema therapy for personality disorders. American Journal of Psychiatry. doi: doi:10.1176/appi.ajp.2013.12040518

Brewin, C. R., Wheatley, J., Patel, T., Fearon, P., Hackmann, A., Wells, A., . . Myers, S. (2009). Imagery rescripting as a brief stand-alone treatment for depressed patients with intrusive memories. Behaviour Research and Therapy, 47(7), 569-576. doi: 10.1016/j.brat.2009.03.008

Bromet, E., Andrade, L., Hwang, I., Sampson, N., Alonso, J., de Girolamo, G., . . Kessler, R. (2011). Crossnational epidemiology of DSM-IV major depressive episode. BMC Medicine, 9(1), 90.

Brown, G. W., Craig, T. K. J., Harris, T. O., Handley, R. V., \& Harvey, A. L. (2007). Development of a retrospective interview measure of parental maltreatment using the Childhood Experience of Care and Abuse (CECA) instrument -- A life-course study of adult chronic depression -- 1. Journal of Affective Disorders, 103(1-3), 205-215. doi: 10.1016/j.jad.2007.05.022

Brown, G. W., \& Moran, P. (1994). Clinical and psychosocial origins of chronic depressive episodes: I. A community survey. British Journal of Psychiatry, 165(4), 447-456. doi: 10.1192/bjp.165.4.447

Broyd, S. J., Demanuele, C., Debener, S., Helps, S. K., James, C. J., \& Sonuga-Barke, E. J. (2009). Default-mode brain dysfunction in mental disorders: a systematic review. Neuroscience and Biobehavioral Reviews, 33(3), 279-296. doi: 10.1016/j.neubiorev.2008.09.002

Carter, J. D., McIntosh, V. V., Jordan, J., Porter, R. J., Frampton, C. M., \& Joyce, P. R. (2013). Psychotherapy for depression: a randomized clinical trial comparing schema therapy and cognitive behavior therapy. Journal of Affective Disorders, 151(2), 500-505. doi: 10.1016/j.jad.2013.06.034

Cuijpers, P., Dekker, J., Hollon, S. D., \& Andersson, G. (2009). Adding psychotherapy to pharmacotherapy in the treatment of depressive disorders in adults: a meta-analysis. J Clin Psychiatry, 70(9), 1219-1229. doi: 10.4088/JCP.09r05021

Cuijpers, P., Karyotaki, E., Weitz, E., Andersson, G., Hollon, S. D., \& van Straten, A. (2014). The effects of psychotherapies for major depression in adults on remission, recovery and improvement: A metaanalysis. Journal of Affective Disorders, 159, 118-126. doi: http://dx.doi.org/10.1016/j.jad.2014. 02.026

Cuijpers, P., Sijbrandij, M., Koole, S. L., Andersson, G., Beekman, A. T., \& Reynolds, C. F., 3rd. (2013). The efficacy of psychotherapy and pharmacotherapy in treating depressive and anxiety disorders: a meta-analysis of direct comparisons. World Psychiatry, 12(2), 137-148. doi: 10.1002/wps.20038

Cuijpers, P., van Straten, A., Andersson, G., \& van Oppen, P. (2008). Psychotherapy for depression in adults: A meta-analysis of comparative outcome studies. Journal of Consulting and Clinical Psychology, 76(6), 909-922. doi: 10.1037/a0013075 10.1037/a0013075. 
Cuijpers, P., van Straten, A., Schuurmans, J., van Oppen, P., Hollon, S. D., \& Andersson, G. (2010). Psychotherapy for chronic major depression and dysthymia: A meta-analysis. Clinical Psychology Review, 30(1), 51-62. doi: 10.1016/j.cpr.2009.09.003

Cuijpers, P., van Straten, A., Warmerdam, L., \& Andersson, G. (2009). Psychotherapy versus the combination of psychotherapy and pharmacotherapy in the treatment of depression: a metaanalysis. Depress Anxiety, 26(3), 279-288. doi: 10.1002/da.20519 [doi]

Dickhaut, V., \& Arntz, A. (2014). Combined group and individual schema therapy for borderline personality disorder: A pilot study. Journal of Behavior Therapy and Experimental Psychiatry, 45(2), 242-251. doi: http://dx.doi.org/10.1016/j.jbtep.2013.11.004

Farrell, J. M., Shaw, I. A., \& Webber, M. A. (2009). A schema-focused approach to group psychotherapy for outpatients with borderline personality disorder: A randomized controlled trial. Journal of Behavior Therapy and Experimental Psychiatry, 40(2), 317-328. doi: 10.1016/j.jbtep.2009.01.002

Ferrari, A. J., Charlson, F. J., Norman, R. E., Patten, S. B., Freedman, G., Murray, C. J. L., . . Whiteford, H. A. (2013). Burden of Depressive Disorders by Country, Sex, Age, and Year: Findings from the Global Burden of Disease Study 2010. PLoS Medicine, 10(11), e1001547. doi: 10.1371/journal.pmed.1001547

Fostick, L., Silberman, A., Beckman, M., Spivak, B., \& Amital, D. (2010). The economic impact of depression: Resistance or severity? European Neuropsychopharmacology, 20(10), 671-675. doi: http://dx.doi.org/10.1016/j.euroneuro.2010.06.001

Frank, E., Prien, R. F., Jarrett, R. B., Keller, M. B., Kupfer, D. J., Lavori, P. W., . . Weissman, M. M. (1991). Conceptualization and rationale for consensus definitions of terms in major depressive disorder. Remission, recovery, relapse, and recurrence. Archives of General Psychiatry, 48(9), 851-855.

Gelenberg, A. J., Trivedi, M. H., Rush, A. J., Thase, M. E., Howland, R., Klein, D. N., . . Keller, M. B. (2003). Randomized, Placebo-Controlled Trial of Nefazodone Maintenance Treatment in Preventing Recurrence in Chronic Depression. Biological Psychiatry, 54(8), 806-817. doi: 10.1016/S00063223(02)01971-6

Giesen-Bloo, J., van Dyck, R., Spinhoven, P., van Tilburg, W., Dirksen, C., van Asselt, T., ... Arntz, A. (2006). Outpatient Psychotherapy for Borderline Personality Disorder: Randomized Trial of SchemaFocused Therapy vs Transference-Focused Psychotherapy. Archives of General Psychiatry, 63(6), 649-658. doi: 10.1001/archpsyc.63.6.649

Greicius, M. D., Flores, B. H., Menon, V., Glover, G. H., Solvason, H. B., Kenna, H., . . Schatzberg, A. F. (2007). Resting-State Functional Connectivity in Major Depression: Abnormally Increased Contributions from Subgenual Cingulate Cortex and Thalamus. Biological Psychiatry, 62(5), 429-437. doi: http://dx.doi.org/10.1016/j.biopsych. 2006.09.020

Hamilton, J. P., Etkin, A., Furman, D. J., Lemus, M. G., Johnson, R. F., \& Gotlib, I. H. (2012). Functional Neuroimaging of Major Depressive Disorder: A Meta-Analysis and New Integration of Baseline Activation and Neural Response Data. American Journal of Psychiatry, 169(7), 693-703. doi: 10.1176/appi.ajp.2012.11071105

Imel, Z. E., Malterer, M. B., McKay, K. M., \& Wampold, B. E. (2008). A meta-analysis of psychotherapy and medication in unipolar depression and dysthymia. Journal of Affective Disorders, 110(3), 197-206. doi: 10.1016/j.jad.2008.03.018

Keller, M. B., McCullough, J. P., Klein, D. N., Arnow, B., Dunner, D. L., Gelenberg, A. J., . . Zajecka, J. (2000). A comparison of nefazodone, the cognitive behavioral-analysis system of psychotherapy, and their combination for the treatment of chronic depression. The New England Journal of Medicine, 342(20), 1462-1470. doi: 10.1056/NEJM200005183422001

Keller, M. B., \& Shapiro, R. W. (1982). 'Double depression': Superimposition of acute depressive episodes on chronic depressive disorders. American Journal of Psychiatry, 139(4), 438-442.

Khan, A., Faucett, J., Lichtenberg, P., Kirsch, I., \& Brown, W. A. (2012). A Systematic Review of Comparative Efficacy of Treatments and Controls for Depression. PLoS ONE, 7(7), e41778. doi: 10.1371/journal.pone.0041778 
Klein, D. N., Arnow, B. A., Barkin, J. L., Dowling, F., Kocsis, J. H., Leon, A. C., . . Wisniewski, S. R. (2009). Early adversity in chronic depression: clinical correlates and response to pharmacotherapy. Depression \& Anxiety (1091-4269), 26(8), 701-710. doi: 10.1002/da.20577

Kupfer, D. J., Frank, E., \& Phillips, M. L. (2012). Major depressive disorder: new clinical, neurobiological, and treatment perspectives. Lancet, 379(9820), 1045-1055. doi: 10.1016/s0140-6736(11)60602-8

Lemogne, C., Delaveau, P., Freton, M., Guionnet, S., \& Fossati, P. (2012). Medial prefrontal cortex and the self in major depression. Journal of Affective Disorders, 136(1-2), e1-e11. doi: 10.1016/j.jad.2010.11.034

Ley, P., Helbig-Lang, S., Czilwik, S., Lang, T., Worlitz, A., Brücher, K., \& Petermann, F. (2011). Phenomenological differences between acute and chronic forms of major depression in inpatients. Nordic Journal of Psychiatry, 65(5), 330-337. doi: doi:10.3109/08039488.2011.552121

Maddux, R. E., Riso, L. P., Klein, D. N., Markowitz, J. C., Rothbaum, B. O., Arnow, B. A., ... Thase, M. E. (2009). Select comorbid personality disorders and the treatment of chronic depression with nefazodone, targeted psychotherapy, or their combination. Journal of Affective Disorders, 117(3), 174-179. doi: 10.1016/j.jad.2009.01.010

Malogiannis, I. A., Arntz, A., Spiropoulou, A., Tsartsara, E., Aggeli, A., Karveli, S., . . Zervas, I. (2014). Schema Therapy for patients with chronic depression: A single case series study. Journal of Behavior Therapy and Experimental Psychiatry, 45(3), 319-329. doi: http://dx.doi.org/10.1016/j.jbtep.2014.02.003

McCullough, J. P., Jr., Klein, D. N., Borian, F. E., Howland, R. H., Riso, L. P., Keller, M. B., \& Banks, P. L. C. (2003). Group Comparisons of DSM-IV Subtypes of Chronic Depression: Validity of the Distinctions, Part 2. Journal of Abnormal Psychology, 112(4), 614-622. doi: 10.1037/0021-843X.112.4.614

McCullough, J. P., Jr., Klein, D. N., Keller, M. B., Holzer, C. E., 3rd, Davis, S. M., Kornstein, S. G., ... Harrison, W. M. (2000). Comparison of DSM-III-R chronic major depression and major depression superimposed on dysthymia (double depression): validity of the distinction. Journal of Abnormal Psychology, 109(3), 419-427.

Nadort, M., Arntz, A., Smit, J. H., Giesen-Bloo, J., Eikelenboom, M., Spinhoven, P., ... van Dyck, R. (2009). Implementation of outpatient schema therapy for borderline personality disorder with versus without crisis support by the therapist outside office hours: A randomized trial. Behaviour Research and Therapy, 47(11), 961-973. doi: 10.1016/j.brat.2009.07.013

Nejad, A. B., Fossati, P., \& Lemogne, C. (2013). Self-Referential Processing, Rumination, and Cortical Midline Structures in Major Depression. Front Hum Neurosci, 7, 666. doi: 10.3389/fnhum.2013.00666

Nemeroff, C. B., Heim, C. M., Thase, M. E., Klein, D. N., Rush, A. J., Schatzberg, A. F., ... Keller, M. B. (2003). Differential responses to psychotherapy versus pharmacotherapy in patients with chronic forms of major depression and childhood trauma. Proceedings of the National Academy of Sciences of the United States of America, 100(24), 14293-14296. doi: 10.1073/pnas.2336126100

Newton-Howes, G., Tyrer, P., \& Johnson, T. (2006). Personality disorder and the outcome of depression: Meta-analysis of published studies. British Journal of Psychiatry, 188(1), 13-20. doi: 10.1192/bjp.188.1.13

Newton-Howes, G., Tyrer, P., Johnson, T., Mulder, R., Kool, S., Dekker, J., \& Schoevers, R. (2013). Influence of Personality on the Outcome of Treatment in Depression: Systematic Review and Meta-Analysis. Journal of Personality Disorders, 1-17.doi: 10.1521/pedi_2013_27_070

Nordahl, H. M., \& Nysaeter, T. E. (2005). Schema therapy for patients with borderline personality disorder: a single case series. Journal of Behavior Therapy and Experimental Psychiatry, 36(3), 254-264.

Northoff, G. (2007). Psychopathology and pathophysiology of the self in depression -- Neuropsychiatric hypothesis. Journal of Affective Disorders, 104(1-3), 1-14.

Pampallona, S., Bollini, P., Tibaldi, G., Kupelnick, B., \& Munizza, C. (2004). Combined Pharmacotherapy and Psychological Treatment for Depression: A Systematic Review. Archives of General Psychiatry, 61(7), 714-719. doi: 10.1001/archpsyc.61.7.714

Paykel, E. S. (2008). Partial remission, residual symptoms, and relapse in depression. Dialogues in Clinical Neuroscience, 10(4), 431-437. 
Renner, F., van Goor, M., Huibers, M., Arntz, A., Butz, B., \& Bernstein, D. (2013). Short-term group schema cognitive-behavioral therapy for young adults with personality disorders and personality disorder features: Associations with changes in symptomatic distress, schemas, schema modes and coping styles. Behaviour Research and Therapy, 51(8), 487-492. doi: http://dx.doi.org/10.1016/j.brat.2013.05.011

Rhebergen, D., Beekman, A. T., Graaf, R., Nolen, W. A., Spijker, J., Hoogendijk, W. J., \& Penninx, B. W. (2009). The three-year naturalistic course of major depressive disorder, dysthymic disorder and double depression. Journal of Affective Disorders, 115(3), 450-459. doi: 10.1016/j.jad.2008.10.018

Richards, D. (2011). Prevalence and clinical course of depression: a review. Clinical Psychology Review, 31(7), 1117-1125. doi: 10.1016/j.cpr.2011.07.004

Riedel, M., Möller, H.-J., Obermeier, M., Schennach-Wolff, R., Bauer, M., Adli, M., . . . Seemüller, F. (2010). Response and remission criteria in major depression - a validation of current practice. Journal of Psychiatric Research, 44(15), 1063-1068. doi: 10.1016/j.jpsychires.2010.03.006

Riso, L. P., du Toit, P. L., Blandino, J. A., Penna, S., Dacey, S., Duin, J. S., . . Ulmer, C. S. (2003). Cognitive aspects of chronic depression. Journal of Abnormal Psychology, 112(1), 72-80. doi: 10.1037/0021843X.112.1.72

Riso, L. P., Froman, S. E., Raouf, M., Gable, P., Maddux, R. E., Turini-Santorelli, N., . . Cherry, M. (2006). The Long-Term Stability of Early Maladaptive Schemas. Cognitive Therapy and Research, 30(4), 515-529.

Riso, L. P., Miyatake, R. K., \& Thase, M. E. (2002). The search for determinants of chronic depression: A review of six factors. Journal of Affective Disorders, 70(2), 103-116.

Rush, A. J., Fava, M., Wisniewski, S. R., Lavori, P. W., Trivedi, M. H., Sackeim, H. A., . . Niederehe, G. (2004). Sequenced treatment alternatives to relieve depression (STAR*D): rationale and design. Controlled Clinical Trials, 25(1), 119-142.

Rush, A. J., Wisniewski, S. R., Zisook, S., Fava, M., Sung, S. C., Haley, C. L., ... Hollon, S. D. (2012). Is prior course of illness relevant to acute or longer-term outcomes in depressed out-patients? A STAR*D report. Psychological Medicine, 42(06), 1131-1149. doi: doi:10.1017/S0033291711002170

Spijker, J., de Graaf, R., Bijl, R. V., Beekman, A. T., Ormel, J., \& Nolen, W. A. (2002). Duration of major depressive episodes in the general population: results from The Netherlands Mental Health Survey and Incidence Study (NEMESIS). British Journal of Psychiatry, 181, 208-213.

Spijker, J., van Straten, A., Bockting, C. L. H., Meeuwissen, J. A. C., \& van Balkom, A. J. L. M. (2013). Psychotherapy, antidepressants, and their combination for chronic major depressive disorder: A systematic review. The Canadian Journal of Psychiatry / La Revue canadienne de psychiatrie, 58(7), 386-392.

Thase, M. E. (2003). Achieving remission and managing relapse in depression. Journal of Clinical Psychiatry, 64 Suppl 18, 3-7.

Trivedi, M. H., Rush, A. J., Wisniewski, S. R., Nierenberg, A. A., Warden, D., Ritz, L., . . Fava, M. (2006). Evaluation of outcomes with citalopram for depression using measurement-based care in STAR*D: implications for clinical practice. American Journal of Psychiatry, 163(1), 28-40. doi: 10.1176/appi.ajp.163.1.28

van Vreeswijk, M. F., \& Broersen, J. (2006). Schemagerichte Therapie in Groepen. Cognitieve Groepspsychotherapie bij Persoonlijkheidsproblematiek. Handleiding voor Therapeuten. Houten: Bohn Stafleu van Loghum.

van Vreeswijk, M. F., Spinhoven, P., Eurelings-Bontekoe, E. H. M., \& Broersen, J. (2012). Changes in Symptom Severity, Schemas and Modes in Heterogeneous Psychiatric Patient Groups Following Short-term Schema Cognitive-Behavioural Group Therapy: A Naturalistic Pre-treatment and Posttreatment Design in an Outpatient Clinic. Clinical Psychology \& Psychotherapy. doi: 10.1002/cpp.1813

Wang, L., Hermens, D. F., Hickie, I. B., \& Lagopoulos, J. (2012). A systematic review of resting-state functional-MRI studies in major depression. Journal of Affective Disorders, 142(1-3), 6-12. doi: 10.1016/j.jad.2012.04.013 
Wells, K. B., Burnam, M. A., Rogers, W., \& Hays, R. (1992). The course of depression in adult outpatients: Results from the Medical Outcomes Study. Archives of General Psychiatry, 49(10), 788-794. doi: 10.1001/archpsyc.1992.01820100032007

Whiteford, H. A., Degenhardt, L., Rehm, J., Baxter, A. J., Ferrari, A. J., Erskine, H. E., . . Vos, T. (2013). Global burden of disease attributable to mental and substance use disorders: findings from the Global Burden of Disease Study 2010. The Lancet, 382(9904), 1575-1586.

Wiersma, J. E., Hovens, J. G., van Oppen, P., Giltay, E. J., van Schaik, D. J., Beekman, A. T., \& Penninx, B. W. (2009). The importance of childhood trauma and childhood life events for chronicity of depression in adults. Journal of Clinical Psychiatry, 70(7), 983-989.

Young, J. E., Klosko, J., \& Weishaar, M. E. (2003). Schema therapy: A practitioner's guide. New York: Guilford Press. 


\section{PART I}

\section{NEUROIMAGING STUDIES IN CHRONIC DEPRESSION}





\section{CHAPTER 2 \\ Neural correlates of self-referential processing in chronic depression}

Submitted as:

Renner, F., Siep, N., Lobbestael, J., Arntz, A., Peeters, F., \& Huibers, M. (2014). Neural correlates of self-referential processing and implicit self-associations in chronic depression. 


\begin{abstract}
Patients with depression tend to process negative information with regard to the self (i.e. self-referential processing). This fMRI study used a passive self-referential processing paradigm to study schema (in)congruent information processing in patients with chronic major depressive disorder (cMDD) $(n=17)$ and non-patient controls $(n=18)$. Participants were instructed to decide whether a presented word was an existing word or a non-word. Stimuli consisted of positive, negative, negative depression related and neutral personality trait words and non-words. Participants also completed an explicit and an implicit measure of positive and negative self-associations. There was a significant interaction between group and word category in the left medial prefrontal cortex (mPFC) and in the left dorsolateral prefrontal cortex (dlPFC). Non-patient controls had relatively increased activity in the mPFC during processing of negative depression related vs. neutral words whereas patients with cMDD had relatively decreased activity. Non-patient controls had relatively increased dIPFC activity during processing of positive vs. neutral words whereas patients with cMDD had relatively decreased activity. Explicit self-descriptiveness ratings of negative depression related words were negatively correlated with signal change in the $\mathrm{MPFC}$ during processing of negative vs. neutral stimuli and with signal change in the dlPFC during processing of positive vs. neutral stimuli for all participants. Implicit self-associations with negative depression vs. positive stimuli did not differ between the groups and were unrelated to BOLD signal change. Our results suggest that CMDD is characterized by distinct neural processing of emotional self-relevant stimuli in the MPFC and the dIPFC.
\end{abstract}

Keywords: chronic major depressive disorder; neuroimaging; self-referential processing; implicit self-associations 


\section{Introduction}

Major depressive disorder (MDD) is a common mood disorder characterized by sad mood, a loss of interest and other emotional, cognitive and physical disturbances (American Psychiatric Association, 2013). It is estimated that about 20\% of depressed patients do not recover within 2 years and thus develop a chronic depression (for a review see: Hölzel, Härter, Reese, \& Kriston, 2011). Compared to episodic depression, chronic depression is associated with more negative health outcomes, increased service use and higher economic costs (Arnow \& Constantino, 2003; Howland, 1993; Smit et al., 2006; Torpey \& Klein, 2008; Wells, Burnam, Rogers, \& Hays, 1992). Given the negative consequences that are associated with chronic depression it is important to understand the underlying neurological disturbances in chronic depression.

The aim of the current study was to explore the neural correlates of self-referential processing in patients with chronic major depressive disorder (cMDD) and to determine its association with explicit and implicit measures of self-associations. Self-referential processing has been defined as the experience of stimuli "as strongly related to one's own person" (Northoff, 2007, p.3). According to cognitive models of depression, negative beliefs about the self are organized in cognitive schemas (Beck, Rush, Shaw, \& Emery, 1979). When confronted with schema congruent (i.e. negative) information, people with depression tend to process this information with regard to the self (i.e. selfreferential processing). This schema-congruent information processing is believed to operate on an automatic level of information processing (Segal, 1988).

Functional neuroimaging studies in non-patients have shown that self-referential processing relies primarily on the so called cortical midline structures (CMS; for a review see: Northoff et al., 2006). The CMS include the medial prefrontal cortex (mPFC), medial orbitofrontal cortex (MOFC), anterior cingulate cortex (ACC), and the posterior cingulate cortex (PCC). Functional neuroimaging studies in patients with depression have emphasized the important role of CMS abnormalities during self-referential processing (for reviews see: Lemogne, Delaveau, Freton, Guionnet, \& Fossati, 2012; Nejad, Fossati, \& Lemogne, 2013). Lemogne, et al. (2009) found increased activity in the dorsal part of the medial frontal gyrus during self-referential processing (vs. general processing) of both positive and negative words in patients with MDD but not in nonpatient controls (Lemogne et al., 2009). In a similar study, Yoshimura et al. (2010) found increased activity in patients with MDD, when compared to non-patient controls, in the mPFC during self-referential processing of negative words. In another study, Grimm et al. (2009) found increased activity in CMS in non-patient controls during self-referential processing vs. passive viewing of positive and negative pictures whereas patients showed generally lower signal intensities. Thus, whereas some studies found increased activity in mPFC in depressed patients vs. non-patient controls during self-referential processing (Lemogne et al., 2009; Yoshimura et al., 2010) other studies found evidence for an opposite pattern (Grimm et al., 2009). Moreover, results of previous studies were mixed with regard to stimulus type (pictures or words) and stimulus valence (positive, negative). 
The present fMRI study adds three new elements to the existing research of abnormalities in self-referential processing during depression. First, previous neuroimaging studies on self-referential processing in depressed patients have focused on patients with non-chronic MDD. To the best of our knowledge no study to date has determined the neural correlates of self-referential processing in patients with cMDD.

Second, previous neuroimaging studies on self-referential processing in depressed patients primarily relied on explicit processing paradigms. This means that participants in these studies were explicitly instructed to make a judgment regarding the selfrelatedness of a stimulus. One limitation of explicit processing paradigms is that instructing participants to engage in self-referential processing elicits controlled rather than more automatic self-referential processing (Lemogne et al., 2011). However, according to theory, patients with depression tend to automatically process schema congruent information (Segal, 1988). Passive processing paradigms might therefore more closely resemble the way in which depressed patients process positive and negative information. In a passive processing paradigm depressed participants are presented with either schema congruent (negative stimuli) or schema incongruent (neutral or positive stimuli) information. It has been shown that passive processing paradigms are a valid alternative to explicit self-referential processing paradigms (Rameson, Satpute, \& Lieberman, 2010).

Third, in the current study, changes in neural activity during processing of emotional personality traits were related to measures of explicit and implicit selfassociations. The self-relatedness of personality traits can be assessed explicitly by asking participants to evaluate the degree to which a certain personality trait describes the self. Such an approach requires the participant to reflect upon the own self and to make an explicit judgment about the self in relation to the personality trait. Yet, schematic information processing is thought to operate on an automatic, implicit level (Segal, 1988). Compared to explicit self-associations, implicit self-associations might therefore more closely be related to neural correlates of self-referential processing of schema congruent information. Implicit self-associations can be assessed using a single category (self) implicit association test (SC-IAT) (Karpinski \& Steinman, 2006). Recently, a depression specific SC-IAT has been proposed to measure implicit self-associations with negative depression related stimuli (Lemmens et al., 2014). No differences with regard to implicit self-associations between patients with episodic depression and nonpatient controls were found (Lemmens et al., 2014). In another study using an IAT, it has been shown that the duration of a depressive episode (i.e. chronicity) was associated with stronger implicit self-depressed associations in patients with depression (Elgersma, Glashouwer, Bockting, Penninx, \& de Jong, 2013). Thus although one previous study found no differences on the depression specific SC-IAT (Lemmens et al., 2014) we expect that patients with a longer duration of depressive episodes (i.e. chronic depression) have stronger depression specific self-associations on the SC-IAT compared to nonpatient controls, and that this association correlates with the brain responses associated with self-referential processing. 
In sum, this study investigates the neural correlates of self-referential processing in patients diagnosed with cMDD using a passive processing paradigm when compared to matched non-patient controls. In addition, we examined explicit and implicit selfassociations with positive and negative personality traits in the same group of participants. It is hypothesized that 1) patients with cMDD show a differential activation pattern in areas of the brain that have previously been associated with self-referential processing (i.e. CMS) during presentation of schema-congruent (negative and depression-related negative trait words) vs. schema incongruent (neutral or positive trait words) compared to non-patient controls; 2) increased neural activity in brain areas of self-referential processing is positively correlated with explicit and implicit selfassociations of personality traits.

\section{Methods}

\section{Participants}

Participants in this study were initially 19 patients with cMDD, of which one was excluded because of language difficulties. For the remaining 18 cMDD patients 18 nonpatient controls were recruited who were matched on age, gender, education level and left/right-handedness. One left handed patient could not be matched to a left handed non-patient control and was therefore matched to a right handed non-patient control participant. After data acquisition, one cMDD patient had to be excluded because of technical problems with the MRI scans. Thus, 17 cMDD and 18 non-patient controls constituted the final sample. Patients in this study were recruited from a specialized secondary mental health facility in the Netherlands (Riagg Maastricht) as part of a single-case series study testing the effects of schema therapy for chronic depression (Clinicaltrials.gov identifier: NCT01153867). Scanning sessions took place before the intervention phase of the study. Diagnostic status of patients was assessed with the Structured Clinical Interview for DSM-IV axis I disorders (SCID-I) by trained clinicians. Patients were included if they met the DSM-IV criteria for a cMDD and if they had a score of 20 or higher on the Beck Depression Inventory second edition (BDI-II). Patients were excluded if they had a bipolar disorder, a psychotic disorder, alcohol or substance dependence, autism spectrum disorder or if they met criteria for a DSM-IV cluster-A or cluster-B personality disorder as assessed with the SCID-II for DSM-IV personality disorders. One patient with co-morbid borderline personality disorder was included in error but is analyzed here. Patients with acute suicide risk were also excluded. Patients who took antidepressant medications were excluded, unless they were stable on medication for 3 months or longer prior to study intake. Nine (53\%) of the patients with chronic depression took antidepressant medication at the time of the study and were 
stable on medication (at least 3 months prior to the start of the study) ${ }^{1}$. Eligible participants with MRI contra-indications were excluded during recruitment.

Non-patient control participants were recruited via posters and via a participant database. Diagnostic status of non-patient controls was assessed with the SCID-I interview by a trained interviewer (FR). Non-patient controls were excluded if they met the DSM-IV criteria for a current axis-I disorder or any lifetime mood disorder. All participants received verbal and written information about the study and signed written informed consent. The study protocol was approved by the medical ethical committee of the University Hospital Maastricht, the Netherlands.

Table 1 provides an overview of the demographic and clinical characteristics of the sample. There were no statistically significant differences on any of the demographic variables between the two groups. The average BDI-II score of patients was 30.47 (SD = 9.68), indicating severe depression. The average BDI-II score of non-patient controls was 2.67 (SD = 2.77) (Table 1).

Table 1. Demographic and clinical characteristics of the sample.

\begin{tabular}{lllll}
\hline Measure & $\begin{array}{l}\text { Patients } \\
(N=17)\end{array}$ & $\begin{array}{l}\text { Controls } \\
(N=18)\end{array}$ & $\begin{array}{l}t \text {-test } \\
(p \text {-value })\end{array}$ & $\begin{array}{l}\chi 2 \\
(p \text {-value })\end{array}$ \\
\hline $\begin{array}{l}\text { Age, } \mathrm{M}(\mathrm{SD}) \\
\begin{array}{l}\text { Gender, } \mathrm{n} \\
\text { female } \\
\text { male }\end{array}\end{array}$ & $40.41(17.77)$ & $42.67(18.73)$ & .72 & - \\
$\begin{array}{l}\text { Education level, n (\%) } \\
\quad \text { low } \\
\text { medium }\end{array}$ & 12 & 13 & - & .92 \\
$\quad$ high & 5 & 5 & & \\
BDI-II score & 5 & 2 & - & .80 \\
\hline
\end{tabular}

Note. BDI-II = Beck Depression Inventory second edition.

\section{Materials and Procedure}

\section{Word stimuli}

The word stimuli for the self-referential processing task were selected from Andersons's list of personality trait words (Anderson, 1968) and from words that have previously been used in a SC-IAT for depression, designed to measure implicit depressive selfassociations (Lemmens et al., 2014). All selected words were rated during a pilot test in an independent sample $(N=13)$ on valence and arousal on a scale ranging from 1 to 9 , with higher scores representing more negative valence and less arousal, respectively (see Table 2). Based on the mean valence and arousal ratings during the pilot test, 80

\footnotetext{
${ }^{1}$ Medication dosage of one patient taking venlafaxine was increased from $75 \mathrm{mg}$. to $150 \mathrm{mg}$. two month prior to start of the study.
} 
personality trait words were selected and categorized into four equal unambiguous categories: 1) positive words (e.g. happy; pleasant; brilliant); 2) general negative words (e.g. arrogant; inattentive; dishonest); 3) depression related negative words (e.g. worthless; guilty; indecisive); 4) neutral words (e.g. moderate; casual; objective); ${ }^{2}$ During pilot testing, general negative and negative depression related word stimuli were rated as more negative compared to neutral and positive words (all $p$-values < .05). Valence ratings of negative and negative depression related words did not differ significantly $(p=.27)$. Selected words from the different categories were matched on the average word length. Although care was taken to also match word stimuli from the different categories on arousal levels as closely as possible, the selected neutral words were rated as less arousing in the pilot test compared to the positive and negative words $(p<.01$ and $p<.001$, respectively). Negative words were rated as slightly more arousing compared to negative depression related words $(p<.01)$. In addition to the personality trait words, participants were presented with 20 non-words which consisted of a random composition of letters. Non-words were included to keep the participant focused on the task. The participant was instructed to press a button when a non-word was presented.

Table 2. Valence and Arousal ratings of selected word stimuli during pilot testing.

\begin{tabular}{lll}
\hline Word category & Valence M (SD) & Arousal M (SD) \\
\hline Neutral & $4.67(0.92)$ & $5.52(0.94)$ \\
Positive & $2.21(0.42)$ & $4.49(0.63)$ \\
Negative & $7.68(0.58)$ & $4.14(0.72)$ \\
Negative depression related & $7.81(0.49)$ & $5.03(1.00)$ \\
\hline
\end{tabular}

Note. $N=13$; Higher valence scores represent more negative valence ratings; Higher arousal scores represent less arousal.

\section{Questionnaires}

Beck depression inventory second edition - The Beck Depression Inventory second edition (BDI-II) is a 21-item self-report instrument to asses depressive symptom severity during the past two weeks (Beck, Steer, \& Brown, 1996). In the present study the Dutch version was used and depressive symptom severity during the past week was assessed. The reason why symptom severity during the past week instead of the past two weeks was assessed was that patients in this study completed the BDI every week as part of a waiting-list phase of a treatment study that followed the fMRI experiment. The Dutch BDI-II has been shown to have good psychometric properties (van der Does, 2002).

Implicit task - Single-category implicit associations test - A depression specific singlecategory implicit association test SC-IAT (Lemmens et al., 2014) was used in order to

\footnotetext{
${ }^{2} \mathrm{~A}$ list of all 80 personality trait words with valence and arousal ratings can be requested from the corresponding author.
} 
assess implicit negative self-associations. The task started with a practice block in which 12 positive and 12 negative words (attributes) were presented, followed by two test blocks. The test blocks included a third word category (self-stimuli). During the test blocks the self-stimuli shared a response key with either the positive or the negative attributes (counterbalanced). Each test block started with 12 practice trials ( 7 attributes and 5 self-stimuli). The practice trial was followed by the presentation of 72 critical trials consisting of 30 -self-stimuli and 43 attributes. Six negative stimuli were selected to reflect main themes in depression (rejected, harmful, worthless, redundant, helpless, and vulnerable) and positive stimuli represented their counterparts (beloved, pleasant, powerful, successful, perfect, and valuable). In addition six self-stimuli were selected: I, me, self, my, own, mine. A more detailed description of the task design is provided elsewhere (Lemmens et al., 2014).

\section{fMRI passive self-referential processing task design and stimulation protocol}

This study used a 2 (group: non-patient controls, cMDD) X 5 (word type: positive, negative general, negative depression related, neutral, non-word) between-subject design. Personality trait words (positive, negative general, negative depression related or neutral) and non-words were presented in a slow-event related design with a presentation duration of $4000 \mathrm{~ms}$. Word categories were presented in a fixed order, which differed per run, with personality trait words within categories and non-words presented randomly. Stimuli presentation was separated by a fixation cross with a random presentation duration of 2000, 3000, 4000 or $5000 \mathrm{~ms}$. The participant was instructed to press a button whenever a non-word appeared. The task consisted of two runs with a duration of 12 min per run. The order of runs was counterbalanced between participants. In each run 100 words were presented (20 from each category). In the second run the same stimuli as in the first run were presented but in a different random order. Thus in total 200 word stimuli were presented during the session. Task instructions and stimuli were presented using E-Prime and projected on a screen behind the scanner that participants viewed through an angled mirror.

\section{Procedure}

Before the first run, participants rated their current mood state along four $100 \mathrm{~mm}$ VAS. Prior to each run, participants were instructed to read each word and to press a button with the left index finger when they detect a non-word. The purpose of this task instruction was to keep participants focused. As part of the experimental procedure, participants also underwent two resting state scans and a diffusion tensor imaging scan (results reported separately). Overall, the scanning session lasted about 60 min. Immediately after the scanning session non-patient control participants completed the SC-IAT. Patients completed the SC-IAT on a different day prior to the scanning session as part of a larger test battery. Following the scanning session, participants were asked to indicate how nervous they felt prior to the scanning session and how good they were able to concentrate during the scanning sessions on two $100 \mathrm{~mm}$ VAS. Participants were 
also asked to rate the valence of each personality trait word and to indicate how much each personality trait word described themselves on a scale from 1 to 5 .

\section{fMRI Data acquisition}

Anatomical images were acquired using a Magnetom Allegra 3T scanner (Siemens Healthcare, Netherlands) located at the Faculty of Psychology and Neuroscience, Maastricht University with a T1-weighted gradient echo (196 slices, TR $=2250 \mathrm{~ms}$, TE = $26 \mathrm{~ms}$, flip angle $=90^{\circ}$, field of view $=256 \mathrm{~mm}$ and voxel dimensions $1 \times 1 \times 1 \mathrm{~mm} 3$ ). Two functional runs were obtained with a T2*-weighted echo planar imaging sequence consisting of 32 slices with a $3 \mathrm{~mm}$ slice thickness. TR was set at $2000 \mathrm{~ms}$, with an TE of $30 \mathrm{~ms}$ and a flip angle of $90^{\circ}$. The field of view was $192 \mathrm{~mm}$ with a voxel size of $3 \times 3 \mathrm{~mm}$ isotropic resolution. During each run 380 volumes were acquired. A slice tilt correction of -30 was used to minimize inhomogeneity artefacts (Weiskopf, Hutton, Josephs, \& Deichmann, 2006).

\section{fMRI data preprocessing}

Data pre-processing and analyses were conducted with Brainvoyager QX v2.4 (Brain Innovations, Maastricht, The Netherlands). The first two volumes of the functional images were omitted due to an unstable signal. For the functional data a slice scan timing correction was performed by sinc interpolation and the data was motion corrected using a 3D motion correction with sinc interpolation and high-pass temporal filtering. The data was smoothed using a $4 \mathrm{~mm}$ full-width-at-half-maximum Gaussian filter. The skull was removed by an automated brain peeling procedure. Functional images were then co-registered to structural images and subsequently normalized using the Talairach and Tournoux transformation procedure.

\section{fMRI analyses}

Data analyses were conducted in three steps. First, individual participants' BOLD signals were modeled in an event-related design using a general linear model (GLM) with 5 predictors, denoting the personality trait words in either positive, negative general, negative depression related, neutral or non-words. Conditions in the GLM were modeled as boxcar functions and convolved with a 2-gamma hemodynamic response function. First-level GLM analyses conducted on individual participants' fMRI signal were submitted to a second-level random effects analysis, treating participants as a random factor in order to identify a network of brain regions involved in processing of personality trait words in general. In these analyses all personality trait words were contrasted with the non-words. A resulting map of brain regions indicating positive activity was selected at a conservative False Discovery Rate (FDR) of 0.005. Based on the resulting map a VTC mask was created and subsequent analyses focused on group X word type interactions within this mask. Volumes-of-interest (VOIs) resulting from the 
group X word type interaction analysis were identified and defined within this map, using a cluster size threshold of 3 and a significance level of $p<.025$. Using this approach a total of 2 VOIs were identified (see Table 3). Within each VOI the beta weights were averaged across participants for each category of word-stimuli (positive, negative general, negative depression related, neutral). To further explore the group X word type interactions in order to investigate our hypothesis, VOI beta weights were exported to the statistical package SPSS version 21. In these simple effects analyses a Bonferroni adjusted significance level alpha (.05/3) was used to avoid inflation of the Type-I error rate. To visualize group $\mathrm{X}$ word type interactions bar-plots were created for each VOI displaying the mean signal change of critical word stimuli vs. neutral words for each group.

Table 3. Areas showing significant personality group X word type interactions

\begin{tabular}{lllll}
\hline Brain region & L/R & $\begin{array}{l}\text { Talairach } \\
\text { coordinates }(\mathrm{x}, \mathrm{y}, \mathrm{z})\end{array}$ & BA & $\begin{array}{l}\text { Cluster size } \\
\text { (voxels) }\end{array}$ \\
\hline Medial prefrontal cortex & $\mathrm{L}$ & $-8,60,10$ & 10 & 111 \\
Premotor cortex / dorsolateral prefrontal cortex & $\mathrm{L}$ & $-25,17,39$ & 8 & 139 \\
\hline
\end{tabular}

Note. $\mathrm{L} / \mathrm{R}=$ Left $/$ Right; $\mathrm{BA}=$ Brodmann area; Talairach coordinates refer to the center of gravity of each VOI.

Analyses of implicit and explicit self-associations

The SC-IAT effect was analyzed using the improved d-score algorithm (Greenwald, Nosek, \& Banaji, 2003). Higher scores on the SC-IAT represent stronger self-associations with positive words than with negative words. To test for differences in explicit and implicit self-associations between the two groups, independent sample $t$-tests were used.

\section{Results}

\section{Subjective measures}

\section{Post session questionnaire}

After the scanning sessions participants were asked to answer questions regarding their experiences during scanning. In this questionnaire participants were asked to rate on $100 \mathrm{~mm}$ VAS how nervous they were prior to the experiment $(0=$ very nervous $-100=$ not nervous at all) and their concentration level during scanning $(0=$ very concentrated - $100=$ not concentrated at all). On average, participants were relatively calm during scanning $(M=71.86, S D=28.13)$ and were able to concentrate on the task $(M=25.43$, $S D=22.72$ ). Depressed patients were significantly more nervous prior to the experiment $(M=58.76, S D=30.13)$ compared to non-patient controls $(M=84.22, S D=19.88 ; t(33)=$ 297, $p=.006$. Participants from the two groups did not differ in their ability to concentrate during the experiment, $t(33)=-1.59, p=.12$. 


\section{Explicit self-associations and valence ratings}

Immediately after the scanning session participants were asked to rate each word that was presented during the experiment on valence and self-relatedness (Figure 1). Patients rated general negative and negative depression related words as significantly more self-descriptive than non-patient controls, $t(33)=-3.48, p=.001$, and $t(33)=-9.09$, $p<.001$, respectively. A significant group X negative word type interaction indicated that patients rated the negative depression related words as more self-descriptive than general negative words, compared to non-patients, $t(33)=6.73, p<.001$. Non-patient controls rated positive trait words significantly more self-descriptive compared to patients, $t(33)=6.22, p<.001$. There were no statistically significant differences in the self-descriptiveness ratings for neutral words, $t(33)=-0.12, p=.91$. The two groups did not differ with respect to the valence ratings. Both patients and non-patient controls rated the general negative and the negative depression related words as more negative than the neutral words, $t(34)=-15.94, p<.001$ and $t(34)=-16.11, p<.001$, respectively. On average, the positive trait words were rated as more positive than the neutral words, $t(34)=13.96, p<.001$.
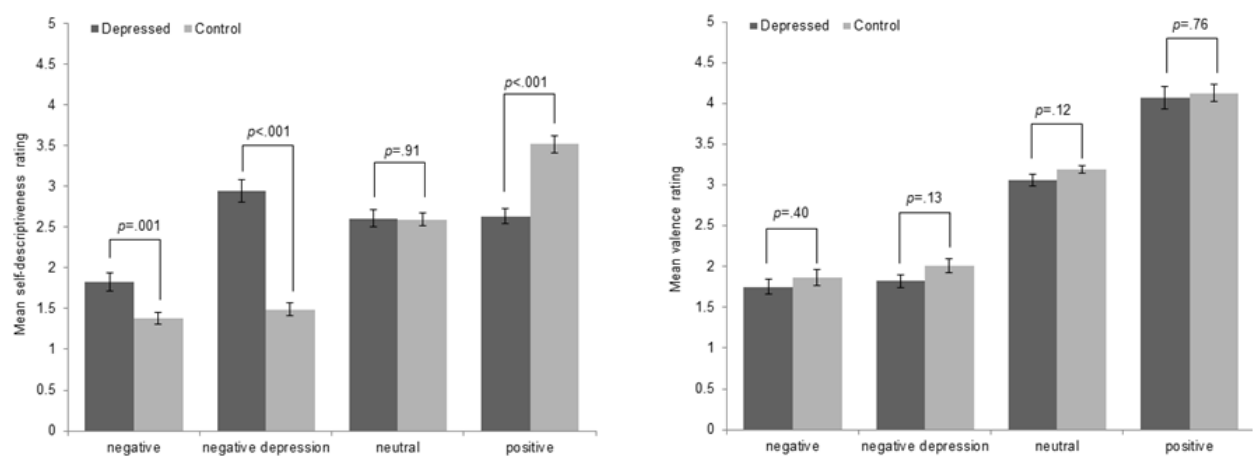

Figure 1. Mean self-descriptiveness (left side) and valence (right side) ratings of general negative, negative depression related, neutral and positive words.

\section{Implicit self-associations}

There were no statistically significant differences between the mean SC-IAT scores of patients $(M=-0.08, S D=0.37)$ and non-patient controls $(M=0.09, S D=0.35 ; t(33)=-$ $1.32, p=.20$ ), indicating that implicit self-associations did not differ between the two groups.

\section{fMRI results}

The whole brain analyses, contrasting personality-trait words with non-words across all participants, revealed a functional network of brain areas as depicted in Figure 2. A VTC mask was created based on this network and subsequent analyses focused on group $\mathrm{X}$ word type interactions within this network. Most brain areas within this network belong 
to the so called cortical midline structures (mPFC, MOFC, PCC) that have previously been associated with self-referential processing. In addition to the CMS the network included parts of the left dorsolateral prefrontal cortex, pre-motor cortex and the left temporal lobe (see Table 4). Within each of these regions the group (non-patient control vs. cMDD) $\mathrm{X}$ word type interactions were subsequently tested. Two regions that showed significant group X word type interactions were identified: mPFC and Premotor cortex/ dlPFC (Table 3).

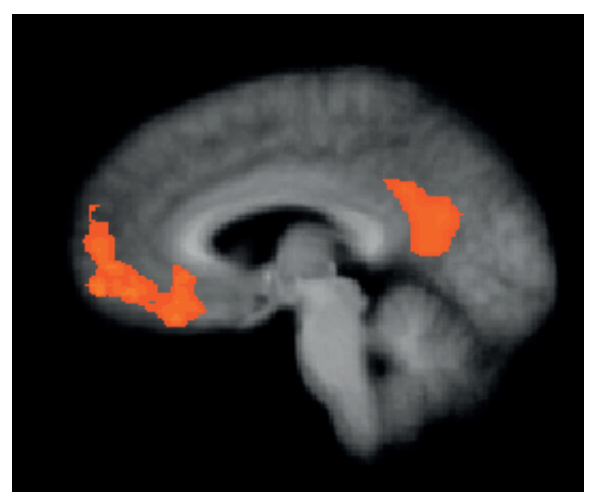

Figure 2. Functional network of brain areas in the cortical midline structures active during processing of personality-trait words vs. nonwords.

Table 4. Areas selected for mask showing significant word X non-word interactions

\begin{tabular}{lllll}
\hline Brain region & L/R & Talairach coordinates $(\mathrm{x}, \mathrm{y}, \mathrm{z})$ & $\mathrm{BA}$ & Cluster size (voxels) \\
\hline Bilateral posterior cingulate cortex & $\mathrm{L} / \mathrm{R}$ & $-4,-55,16$ & 23 & 7152 \\
Ventromedial prefrontal cortex & $\mathrm{L} / \mathrm{R}$ & $-5,44,-1$ & 32 & 6431 \\
Anterior prefrontal cortex & $\mathrm{L}$ & $-11,48,11$ & 10 & 91 \\
Dorsolateral prefrontal cortex & $\mathrm{L}$ & $-24,24,43$ & 8 & 451 \\
Fusiform gyrus & $\mathrm{L}$ & $-32,-36,-10$ & 37 & 95 \\
Middle temporal gyrus & $\mathrm{L}$ & $-44,-72,24$ & 39 & 406 \\
Superior temporal gyrus & $\mathrm{L}$ & $-47,14,-26$ & 38 & 252 \\
Inferior temporal gyrus & $\mathrm{L}$ & $-62,-5,-17$ & 21 & 818 \\
\hline
\end{tabular}

\section{Medial prefrontal cortex}

Within the left medial prefrontal cortex (mPFC), there was a significant interaction between group and word type, $F(3,31)=3.38, p=.031$. Subsequent tests contrasting each category of emotional personality trait words with neutral words, between and within groups, were conducted at a Bonferroni adjusted significance level alpha of .016 $(.05 / 3)$. Thus only $p$-values $<.016$ were considered statistically significant. Non-patient controls had a greater BOLD signal in the left mPFC during processing of negative depression related vs. neutral word stimuli whereas patients with cMDD had a decreased BOLD response during processing of negative depression related vs. neutral word stimuli, $F(1,33)=10.38, p=.003$. This group difference was also reflected in a 
significant negative correlation between BOLD signal changes in negative depression related vs. neutral words and the BDI-II total score $(r=-.42, p=.011)$, showing that higher depressive symptom severity, across all participants, was associated with decreased BOLD signal for this contrast. The BOLD signal of the two groups did not differ significantly during processing of positive vs. neutral stimuli, $F(1,33)=3.65, p$ $=.064$ or during processing of general negative vs. neutral word stimuli, $F(1,33)=5.78$, $p=.022$ (see Figure 3). Within group comparisons of emotional personality trait words vs. neutral words revealed that non-patient controls showed an increased BOLD signal in the left $\mathrm{mPFC}$ during processing of negative depression related vs. neutral personality trait words, $t(18)=-2.93, p=.009$. The other within group contrasts for non-patient control participants were not significant (all $p$-values $>.016$ ). Within the group of depressed patients none of the within group contrasts were significant (all $p$ values $>.016$ ). Moreover, post-hoc tests revealed that the group $\mathrm{x}$ negative word type (general negative vs. depression related words) contrast failed to reach significance, $F(1$, $33)=0.02, p=.876$, suggesting that the evidence for the specificity of the effect for negative depression words is mixed. Similarly, the group $\mathrm{x}$ positive vs. negative depression words contrast failed to reach significance, $F(1,33)=0.79, p=.381$.

\section{Premotor cortex / dorsolateral prefrontal cortex}

In the following the premotor cortex/dorsolateral prefrontal cortex VOI will be referred to as dIPFC. The interaction between word stimuli and group within the left dlPFC was significant, $F(3,31)=3.19, p=.037$. Subsequent tests contrasting each category of emotional personality trait words with neutral words, between and within groups, were conducted at a Bonferroni adjusted significance level alpha of .016 (.05/3). Thus only $p$ values $<.016$ were considered statistically significant. Non-patient controls showed a greater BOLD signal in the left dIPFC during processing of positive vs. neutral word stimuli whereas patients with cMDD had a decrease in BOLD signal during processing of positive vs. neutral word stimuli, $F(1,33)=6.72, p=.014$. This group difference was also reflected in a significant correlation between BOLD signal changes in positive vs. neutral words and the BDI-II total score $(r=-.40, p=.016)$, showing that higher BDI-II scores were associated with decreased BOLD signal for this contrast. The BOLD signal of the two groups did not differ statistically significantly during processing of general negative vs. neutral stimuli, $F(1,33)=0.79, p=.380$ or during processing of negative depression vs. neutral word stimuli, $F(1,33)=3.20, p=.083$ (see Figure 3 ). Within group comparisons of emotional personality trait words vs. neutral words for the nonpatient control group were not significant (all $p$-values $>.016$ ). Within the group of depressed participants none of the within group contrasts were significant (all $p$ values $>$.016). Although the groups differed significantly only for the positive vs. neutral words, the evidence for the specificity of the effect for positive words was limited as the groups did not differ significantly on the positive vs. negative depression word categories, $F(1,33)=0.89, p=.887$.

Parameter estimates for BOLD signal changes in emotional words vs. neutral word contrasts within the $\mathrm{mPFC}$ and the dIPFC were correlated with the concentration ability 
ratings, nervousness ratings, the mean mood ratings prior to the task and the word valence and self-descriptiveness ratings. In order to avoid an inflation of the Type-I error rate in these analyses only correlations within contrasts that were significant in the initial analyses were investigated. Thus, correlations between BOLD signal change during processing of negative depression related words vs. neutral words and these variables were evaluated within the MPFC and correlations between BOLD signal change during processing of positive vs. neutral words and these variables were evaluated within the dlPFC.

Within the mPFC the self-descriptiveness ratings of negative depression related words were negatively correlated with BOLD signal changes during processing of negative depression related vs. neutral words $(r=-.34, p=.045)$. This shows that explicit ratings of negative depression words as self-descriptive, across all subjects, were associated with decreased BOLD signal during processing of negative depression related words vs. neutral words. All other variables were unrelated to BOLD signal changes (all $p$-values $>.05)$.

\section{Correlations between subjective measures and BOLD signal change}

Within the dlPFC there was a significant negative correlation between selfdescriptiveness ratings of negative and negative depression related words and BOLD signal change during processing of positive vs. neutral word stimuli $(r=-.40, p=.018$ and $r=-.46, p=.005$ ). This shows that explicit ratings of negative (depression related) words as self-descriptive, across all subjects, were associated with decreased BOLD signal during processing of positive vs. neutral words. All other variables were unrelated to BOLD signal changes (all $p$-values $>.05$ ).

Results of additional correlational analyses, including associations between explicit and implicit self-associations as well as correlations between BOLD signal change in the MPFC and the DLPFC are provided in the online supplemental material.

\section{Correlations between implicit self-associations and BOLD signal change}

Implicit self-associations did not correlate statistically significantly with parameter estimates for BOLD signal change in the mPFC or in the dlPFC during processing of emotional vs. neutral words (all $p$-values $>.05$ ).

\section{Correlations between use of antidepressants and BOLD signal change}

About half $(53 \%)$ of the patients in this study took antidepressant medication at the time of the study. None of the within group contrasts of BOLD signal change for emotional vs. neutral words in the MPFC and dPFC were correlated with antidepressant medication use (all $p$-values $>.05$ ). 

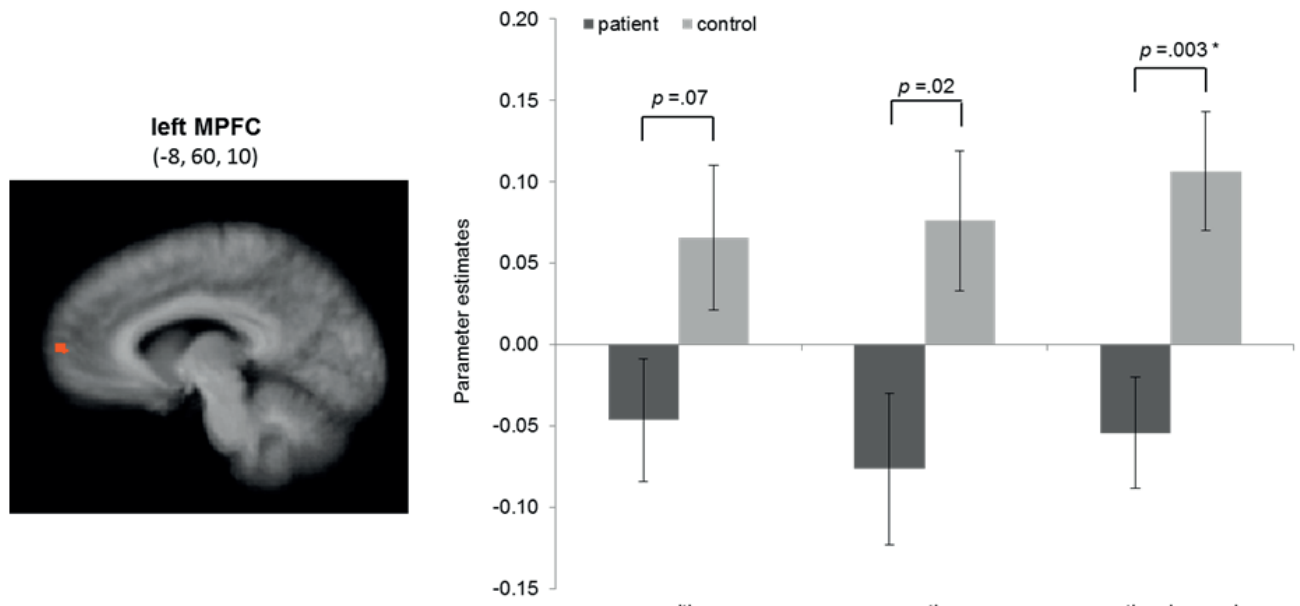

left premotor cortex / DLPFC
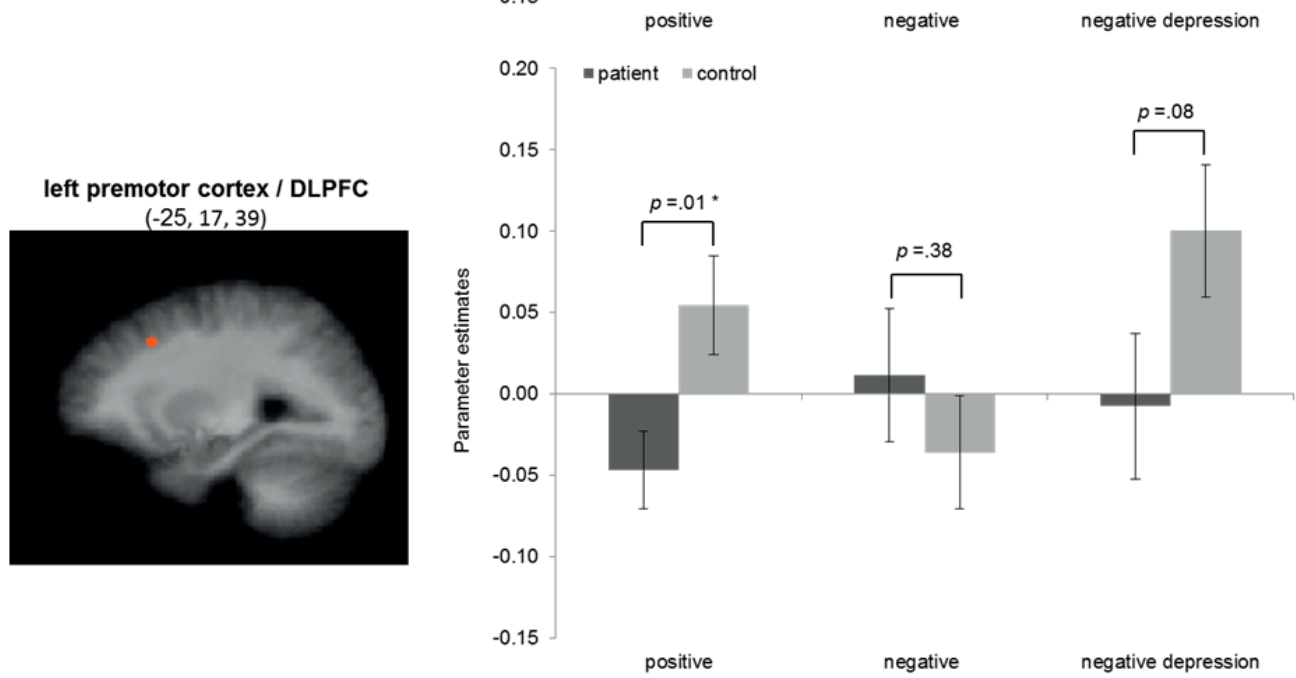

Figure 3. Sagittal view displaying the left mPFC (upper display) and the left premotor cortex / dlPFC (lower display). The bar chart on the right of the image displays parameter estimates between the two groups for each word category relative to neutral words. The error bars represent the standard error of the mean.

Note. ${ }^{*}=$ statistical significant at a Bonferroni adjusted significance level alpha of .016 .

\section{Discussion}

The current study is the first to examine the neural correlates of self-referential processing in patients with cMDD. We used functional Magnetic Resonance Imaging (fMRI) during the presentation of positive, general negative, negative depression related, and neutral personality trait words. Furthermore, we assessed explicit and implicit selfassociations with positive and negative depression related personality trait words in patients with cMDD and in non-patient controls. 
On the neural level, processing of negative depression related words vs. neutral words was associated with relatively more neural activity in the left medial prefrontal cortex (mPFC) in non-patient controls and with relatively lower neural activity in patients with cMDD. Moreover, explicit, but not implicit, self-associations with negative depression words were associated with decreased BOLD signal change in the mPFC during processing of negative depression related words. The mPFC belongs to the so called cortical midline structures and has previously been associated with selfreferential processing (Northoff, 2007; Northoff et al., 2006). Previous neuroimaging studies in depressed patients and non-patient controls have either reported decreased (Grimm et al., 2009) or increased activity in the mPFC during self-referential processing (Lemogne et al., 2009; Yoshimura et al., 2010). It has been argued that the decreased activity in the mPFC in depressed patients during self-referential processing, that was also found in the current study, might reflect increased resting state activity in depressed patients such that task stimuli do not induce strong signal changes in patients (Grimm et al., 2009). In line with this explanation, it has been shown, for example, that increased resting state activity in the ventromedial prefrontal cortex in patients with depression compared to non-patient controls reduces less while looking at negative pictures (Sheline et al., 2009).

The evidence for the stimulus-specificity of the decreased signal strength during processing of emotional words vs. neutral words in patients vs. non-patient controls in the $\mathrm{mPFC}$ for negative depression related personality words vs. neutral words was mixed. Only this word contrast reached Bonferroni-corrected significant difference between groups. However, groups did not differ significantly as to the negative depression vs. general negative words and negative depression vs. positive words contrasts. The negative depression related words that were used in the present study describe core themes in depression. Reduced stimuli induced activity by these words in depressed patients might reflect a heightened resting state activity in brain areas that are activated by these stimuli in non-patient subjects (Grimm et al., 2009). In line with this, it has been argued that control tasks that are not very demanding (e.g. passive viewing) are more likely to allow for spontaneous self-referential processing to occur (Lemogne et al., 2012). Given the non-demanding control task in the current study, it is possible that patients with cMDD in the current study engaged in spontaneous selfreferential processing during the control task such that negative depression related stimuli did not induce a signal change in the mPFC. The mixed evidence for word specificity might however also indicate that there was a more general blunting, a possibility that is discussed later, with blunting perhaps increased in the negative words specifically related to depression.

There was a significant interaction between word category and group in the left dorsolateral prefrontal cortex (dlPFC). Processing of positive personality trait words vs. neutral words was associated with a relative increased neural activity in the dlPFC in non-patient controls and with a relative decrease in neural activity in patients with cMDD. In line with these findings, one previous neuroimaging study in patients with a milder form of chronic depression (dysthymic disorder) found hypoactivity in the dlPFC 
compared to non-patient controls during processing of positive pictures (Ravindran et al., 2009). It has been suggested that reduced activity in the dlPFC during processing of positive vs. neutral stimuli might reflect decreased attention towards positive stimuli in patients with depression (Ravindran et al., 2009). In line with these findings, stimulation of the left dlPFC with repetitive Transcranial Magnetic Stimulation results in decreased depressive symptoms in patients with treatment-resistant depression (Avery et al., 2006; Fitzgerald et al., 2003; Pascual-Leone, Rubio, Pallardó, \& Catalá, 1996) and there is evidence that dIPFC activity in patients with depression increases during treatment with antidepressants (Fitzgerald, Laird, Maller, \& Daskalakis, 2008). It should be noted however that the evidence for the word category specificity of the dIPFC response was limited, as the response to positive words when compared to other emotional word categories did not differ significantly between groups. This might hint at a more general blunting of dIPFC responses during processing of emotional words in cMDD.

In the present study, depressive symptom severity was negatively associated with BOLD signal changes in the $\mathrm{mPFC}$ during processing of negative depression related personality words vs. neutral words. Moreover, there was a negative correlation between depressive symptom severity and BOLD signal changes in the dlPFC during processing of positive vs. neutral words. This finding is in line with one previous study demonstrating negative correlations between neural activity in the dorsomedial prefrontal cortex and depressive symptom severity during self-referential processing (Grimm et al., 2009). It should be noted, that these correlations do not necessarily imply that BOLD signal changes in these areas fluctuate on a continuum with depressive symptom severity. The correlations with depressive symptom severity should rather be seen as an additional test of the group differences in BOLD signal changes were one group had very low depressive symptom severity (non-patient controls) and the other group high depressive symptom severity (cMDD).

The finding of the current study that processing of emotional relative to neutral words was generally associated with decreased BOLD signal change in patients suggests that patients with chronic depression show a general blunting response when confronted with emotional stimuli. It has been argued previously that MDD is characterized by blunted emotional reactivity, not only to positive emotional stimuli but also towards negative emotional stimuli (Rottenberg, Gross, \& Gotlib, 2005). This is also referred to as the emotion-context insensitivity hypothesis (Rottenberg et al., 2005). This hypothesis has been supported in a recent meta-analysis (Bylsma, Morris, \& Rottenberg, 2008) in patients with depression for self-reported emotions, as well as for physiological emotional response indicators (Bylsma et al., 2008). Blunted emotional responses to everyday negative stressors have also been associated with lower recovery from depression at 18 month follow-up thus depression chronicity (Peeters, Berkhof, Rottenberg, \& Nicolson, 2010). Moreover, it has been shown that depressed patients show blunted response pattern towards positive and negative words during a lexical decision task in brain areas related to language processing (Canli et al., 2004). Although our findings of decreased signal strength in patients with cMDD in the left MPFC were only significant for negative depression related words, it should be noted that the 
direction of signal change between the two groups was similar for positive and negative words, and posthoc comparisons between the emotional word categories did not support word specificity (Figure 3). The fact that the pattern was similar for positive and general negative words further support the idea that patients with chronic depression show a blunted response to all emotional stimuli although this might be more pronounced for negative depression related stimuli. It has been argued previously that findings like this support the hypothesis that depressive mood has inhibitory effects on emotional processing (Rottenberg, 2007).

Different explanations might account for differences between previous studies assessing neural correlates of self-referential processing in depression and the results of the current study. Previous studies used explicit paradigms to study neural correlates of self-referential processing. In these studies, participants were explicitly instructed to process stimuli with regard to the self. In contrast, in the current study a passive processing paradigm was used to allow for a more "automatic" self-referential processing during the presentation of schema congruent personality trait words. The subjective word evaluation in the current study indicated that, on an explicit level, depressed patients indeed described negative depression related personality trait words as more self-descriptive (i.e. schema congruent), whereas non-patient controls described positive personality words as more self-descriptive. The current paradigm therefore seems to be well suited to study the more automatic aspects of self-referential processing in depressed patients. Two previous studies have shown that passive processing of self-relevant information relies on the same neural structures as selfreferential processing in explicit paradigms (Moran, Heatherton, \& Kelley, 2009; Rameson et al., 2010). It is therefore unlikely that differences in the experimental paradigm account for differences with findings of previous studies.

Another explanation for divergent findings might be that previous studies included patients with depression in general without a specific focus on patients with cMDD (for a review see: Lemogne et al., 2012). It is possible that due to the long duration of depression (i.e. more than 2 years), patients with cMDD display a more emotionally numbing response toward emotional stimuli.

On an explicit, or subjective level, we found that patients with cMDD rated negative depression related personality traits as most self-descriptive whereas non-patient controls rated positive personality traits as most self-descriptive. On an implicit level there were no differences between the two groups with regard to negative selfassociations. These latter findings are in line with one previous study that used the same depression specific SC-IAT that was used in the current study (Lemmens et al., 2014). Based on previous research showing that implicit depressive-self associations are associated with depressive episode duration (Elgersma et al., 2013), we expected that chronically depressed patients in the current study would show increased implicit negative self-associations compared to non-patient controls that would correlate with brain responses associated with self-referential processing.

Future studies should aim to directly compare implicit negative self-associations in patients with episodic depression and patients with chronic depression. The fact that we 
did not find a difference between the two groups could reflect a power problem since the current study relies on a relatively small sample. In line with this, patients in the current study indeed endorsed more implicit negative self-associations whereas nonpatient controls endorsed more positive implicit self-associations and this difference might have been statistically significant in a larger sample. An alternative explanation for the lack of differences in implicit self-associations is that the two groups completed the SC-IAT at different time points: Non-patient controls completed the SC-IAT after the scanning session thus shortly after having been exposed to the same stimuli in the scanner. Patients completed the SC-IAT on a different day, as part of a treatment study baseline assessment.

Several limitations should be considered when interpreting these results. First, the current study did not include a clinical control group and it therefore remains unclear how specific the findings of the current study are with respect to chronic depression. It would be interesting, for example, to compare patients with cMDD to patients with nonchronic MDD to determine the specificity of the results. Second, about half of the patients in the current study took antidepressant medication at the time when the study was conducted and it is unclear if this has influenced the results of the current study. However, results showed that antidepressant medication use was not associated with the main findings in the current study. Third, the sample size of the current study was relatively small and it was therefore not possible to investigate association between study variables and BOLD signal changes in multivariate analyses. Fourth, the initially intended matching did not succeed completely, as one cMDD participant had to be excluded due to technical problems and one other cMDD participant could not be matched on handiness. Lastly, one cMDD participant suffered from borderline personality disorder which might have influenced the individual's responses.

Clinically, our findings suggest that patients with chronic depression show a blunted response when confronted with emotional stimuli. Emotional processing is an important aspect of emotion focused treatments for depression (Greenberg \& Watson, 2006) and in treatments with a strong experiential component, such as schema therapy (Young, Klosko, \& Weishaar, 2003). We have argued previously, that treatment for chronic depression could benefit from a stronger focus on emotional processing (Renner, Arntz, Leeuw, \& Huibers, 2013). Future studies should investigate the impact of treatment that focusses on emotional processing (e.g. schema therapy) on the neural reactivity to emotional stimuli.

In conclusion, our findings suggest that chronic depression is characterized by a distinct neural processing of emotional self-relevant stimuli in the left mPFC and left dIPFC. The observed pattern might represent an emotional numbing response towards emotional stimuli in general. Future studies should determine the specificity of these findings with respect to chronic depression and the stability of these patterns of neural activity over a course of treatment for depression that has a strong focus on emotional processing techniques such as schema therapy or emotion focused therapy for depression. 


\section{Acknowledgements}

We are grateful to our colleagues at Maastricht University who supported this study: Annie Hendriks and Annie Raven helped with patient recruitment; Danielle Jongen assisted with recruitment of matched controls; Rosanne Janssen developed the infrastructure for online data collection; Anne Roefs programmed the original version of the single-category implicit associations test; Michiel Vestjens programmed an online version of the single-category implicit associations test that was used in the current study. Thanks are also due to our colleagues at the Riagg Maastricht who helped to recruit patients and conducted clinical interviews for this study. 


\section{References}

American Psychiatric Association. (2013). Diagnostic and statistical manual of mental disorders (5th ed.). Arlington, VA: American Psychiatric Publishing.

Anderson, N. H. (1968). Likableness ratings of 555 personality-trait words. Journal of Personality and Social Psychology, 9(3), 272-279.

Arnow, B. A., \& Constantino, M. J. (2003). Effectiveness of psychotherapy and combination treatment for chronic depression. Journal of Clinical Psychology, 59(8), 893-905. doi: 10.1002/jclp.10181

Avery, D. H., Holtzheimer, P. E., 3rd, Fawaz, W., Russo, J., Neumaier, J., Dunner, D. L., . . Roy-Byrne, P. (2006). A controlled study of repetitive transcranial magnetic stimulation in medication-resistant major depression. Biological Psychiatry, 59(2), 187-194. doi: 10.1016/j.biopsych.2005.07.003

Beck, A. T., Rush, A. J., Shaw, B. F., \& Emery, G. (1979). Cognitive therapy of depression. New York: Guilford.

Beck, A. T., Steer, R. A., \& Brown, G. K. (1996). Manual for the Beck Depression Inventory—II. San Antonio, TX.

Bylsma, L. M., Morris, B. H., \& Rottenberg, J. (2008). A meta-analysis of emotional reactivity in major depressive disorder. Clinical Psychology Review, 28(4), 676-691. doi: http://dx.doi.org/10.1016/j.cpr.2007.10.001

Canli, T., Sivers, H., Thomason, M. E., Whitfield-Gabrieli, S., Gabrieli, J. D., \& Gotlib, I. H. (2004). Brain activation to emotional words in depressed vs healthy subjects. Neuroreport, 15(17), 2585-2588.

Elgersma, H. J., Glashouwer, K. A., Bockting, C. L. H., Penninx, B. W. J. H., \& de Jong, P. J. (2013). Hidden scars in depression? Implicit and explicit self-associations following recurrent depressive episodes. Journal of Abnormal Psychology, 122(4), 951-960. doi: 10.1037/a0034933

Fitzgerald, P. B., Brown, T. L., Marston, N. U., Daskalakis, Z., de Castella, A., \& Kulkarni, J. (2003). Transcranial magnetic stimulation in the treatment of depression: A double-blind, placebo-controlled trial. Archives of General Psychiatry, 60(10), 1002-1008. doi: 10.1001/archpsyc.60.9.1002

Fitzgerald, P. B., Laird, A. R., Maller, J., \& Daskalakis, Z. J. (2008). A meta-analytic study of changes in brain activation in depression. Human Brain Mapping, 29(6), 683-695. doi: 10.1002/hbm.20426

Greenberg, L. S., \& Watson, J. C. (2006). Emotion-Focused Therapy for Depression. Washington: American Psychological Association.

Greenwald, A. G., Nosek, B. A., \& Banaji, M. R. (2003). Understanding and using the implicit association test: I. An improved scoring algorithm. Journal of Personality and Social Psychology, 85(2), 197-216.

Grimm, S., Ernst, J., Boesiger, P., Schuepbach, D., Hell, D., Boeker, H., \& Northoff, G. (2009). Increased selffocus in major depressive disorder is related to neural abnormalities in subcortical-cortical midline structures. Human Brain Mapping, 30(8), 2617-2627. doi: 10.1002/hbm.20693

Hölzel, L., Härter, M., Reese, C., \& Kriston, L. (2011). Risk factors for chronic depression -- A systematic review. Journal of Affective Disorders, 129(1-3), 1-13. doi: 10.1016/j.jad.2010.03.025

Howland, R. H. (1993). Chronic depression. Hospital and Community Psychiatry, 44(7), 633-639.

Karpinski, A., \& Steinman, R. B. (2006). The Single Category Implicit Association Test as a measure of implicit social cognition. Journal of Personality and Social Psychology, 91(1), 16-32.

Lemmens, L. H. J. M., Roefs, A., Arntz, A., van Teeseling, H. C., Peeters, F., \& Huibers, M. J. H. (2014). The value of an implicit self-associative measure specific to core beliefs of depression. Journal of Behavior Therapy and Experimental Psychiatry, 45(1), 196-202. doi: http://dx.doi.org/10.1016/j.jbtep.2013.10.006

Lemogne, C., Delaveau, P., Freton, M., Guionnet, S., \& Fossati, P. (2012). Medial prefrontal cortex and the self in major depression. Journal of Affective Disorders, 136(1-2), e1-e11. doi: 10.1016/j.jad.2010.11.034

Lemogne, C., Gorwood, P., Bergouignan, L., Pelissolo, A., Lehericy, S., \& Fossati, P. (2011). Negative affectivity, self-referential processing and the cortical midline structures. Social Cognitive and Affective Neuroscience, 6(4), 426-433. doi: 10.1093/scan/nsq049

Lemogne, C., le Bastard, G., Mayberg, H., Volle, E., Bergouignan, L., Lehéricy, S., . . Fossati, P. (2009). In search of the depressive self: Extended medial prefrontal network during self-referential processing in major depression. Social Cognitive and Affective Neuroscience, 4(3), 305-312. 
Moran, J. M., Heatherton, T. F., \& Kelley, W. M. (2009). Modulation of cortical midline structures by implicit and explicit self-relevance evaluation. Social Neuroscience, 4(3), 197-211. doi: $10.1080 / 17470910802250519$

Nejad, A. B., Fossati, P., \& Lemogne, C. (2013). Self-Referential Processing, Rumination, and Cortical Midline Structures in Major Depression. Front Hum Neurosci, 7, 666. doi: 10.3389/fnhum.2013.00666

Northoff, G. (2007). Psychopathology and pathophysiology of the self in depression -- Neuropsychiatric hypothesis. Journal of Affective Disorders, 104(1-3), 1-14.

Northoff, G., Heinzel, A., de Greck, M., Bermpohl, F., Dobrowolny, H., \& Panksepp, J. (2006). Self-referential processing in our brain--A meta-analysis of imaging studies on the self. Neuroimage, 31(1), 440-457.

Pascual-Leone, A., Rubio, B., Pallardó, F., \& Catalá, M. D. (1996). Rapid-rate transcranial magnetic stimulation of left dorsolateral prefrontal cortex in drug-resistant depression. The Lancet, 348(9022), 233-237. doi: http://dx.doi.org/10.1016/S0140-6736(96)01219-6

Peeters, F., Berkhof, J., Rottenberg, J., \& Nicolson, N. A. (2010). Ambulatory emotional reactivity to negative daily life events predicts remission from major depressive disorder. Behaviour Research and Therapy, 48(8), 754-760. doi: 10.1016/j.brat.2010.04.008

Rameson, L. T., Satpute, A. B., \& Lieberman, M. D. (2010). The neural correlates of implicit and explicit selfrelevant processing. Neuroimage, 50(2), 701-708. doi: http://dx.doi.org/10.1016/j.neuroimage.2009.12.098

Ravindran, A. V., Smith, A., Cameron, C., Bhatla, R., Cameron, I., Georgescu, T. M., \& Hogan, M. J. (2009). Toward a functional neuroanatomy of dysthymia: a functional magnetic resonance imaging study. Journal of Affective Disorders, 119(1-3), 9-15. doi: 10.1016/j.jad.2009.03.009

Renner, F., Arntz, A., Leeuw, I., \& Huibers, M. (2013). Treatment for chronic depression using schema therapy. Clinical Psychology Science and Practice, 20, 166-180.

Rottenberg, J. (2007). Major depressive disorder: Emerging evidence for emotion context insensitivity. (J. Rottenberg \& I. Johnson Eds.). Washington, DC: American Psychological Association.

Rottenberg, J., Gross, J. J., \& Gotlib, I. H. (2005). Emotion Context Insensitivity in Major Depressive Disorder. Journal of Abnormal Psychology, 114(4), 627-639. doi: 10.1037/0021-843X.114.4.627

Segal, Z. V. (1988). Appraisal of the self-schema construct in cognitive models of depression. Psychological Bulletin, 103(2), 147-162.

Sheline, Y. I., Barch, D. M., Price, J. L., Rundle, M. M., Vaishnavi, S. N., Snyder, A. Z., . . Raichle, M. E. (2009). The default mode network and self-referential processes in depression. Proceedings of the National Academy of Sciences, 106(6), 1942-1947. doi: 10.1073/pnas.0812686106

Smit, F., Cuijpers, P., Oostenbrink, J., Batelaan, N., de Graaf, R., \& Beekman, A. (2006). Costs of Nine Common Mental Disorders: Implications for Curative and Preventive Psychiatry. Journal of Mental Health Policy and Economics, 9(4), 193-200.

Torpey, D. C., \& Klein, D. N. (2008). Chronic depression: Update on classification and treatment. Current Psychiatry Reports, 10, 458-464.

van der Does, A. J. W. (2002). Handleiding: De Nederlandse versie van de Beck Depression Inventory, 2de Editie. [The Dutch version of the Beck Depression Inventory, 2nd ed.]. Lisse: Swets and Zeitlinger b.v.

Weiskopf, N., Hutton, C., Josephs, O., \& Deichmann, R. (2006). Optimal EPI parameters for reduction of susceptibility-induced BOLD sensitivity losses: A whole-brain analysis at $3 \mathrm{~T}$ and $1.5 \mathrm{~T}$. Neuroimage, 33(2), 493-504. doi: http://dx.doi.org/10.1016/j.neuroimage.2006.07.029

Wells, K. B., Burnam, M. A., Rogers, W., \& Hays, R. (1992). The course of depression in adult outpatients: Results from the Medical Outcomes Study. Archives of General Psychiatry, 49(10), 788-794. doi: 10.1001/archpsyc.1992.01820100032007

Yoshimura, S., Okamoto, Y., Onoda, K., Matsunaga, M., Ueda, K., Suzuki, S.-i., \& Yamawaki, S. (2010). Rostral anterior cingulate cortex activity mediates the relationship between the depressive symptoms and the medial prefrontal cortex activity. Journal of Affective Disorders, 122(1-2), 76-85.

Young, J. E., Klosko, J., \& Weishaar, M. E. (2003). Schema therapy: A practitioner's guide. New York: Guilford Press. 


\section{Online supplementary material}

\section{Associations between explicit and implicit self-associations}

In the overall sample, implicit self-associations did not correlate with explicit selfassociations of the same positive personality traits $(r=-.1, p=.57)$ or with explicit selfassociations of the same negative depression related personality traits $(r=-.17, p=.32$ ). Within the group of depressed patients there was a significant negative correlation between implicit self-associations and explicit self-associations of positive personality trait words $(r=-.70, p=.002$ ), showing that more positive implicit self-associations in depressed patients were related to less positive explicit self-associations.

\section{Correlations between BOLD signal change in MPFC and DLPFC}

To test whether signal changes between the different brain regions were correlated, the parameter estimates for BOLD signal changes during processing of emotional vs. neutral words in the MPFC and DLPFC were correlated (see Table1 appendix). Within the MPFC signal change during processing of the different word categories was highly positively intercorrelated ( $r$ s between .77-.86; all $p$-values < .001). Similarly, within the DLPFC signal change during processing of the different word categories was highly positively intercorrelated ( $r$ s between .71-.88; all $p$-values $<.001$ ). Bold signal during processing of neutral words in the MPFC was positively correlated with the bold signal in the DLPFC during processing of neutral $(r=.50, p=.002)$ positive $(r=.48, p=.004)$, negative $(r$ $=.37, p=.029)$ and negative depression related $(r=.40, p=.017)$ stimuli. BOLD signal in the MPFC during processing of positive words was significantly associated with BOLD signal in the DLPFC during processing of positive words $(r=.38, p=.026)$.

\section{Appendix Table 1}

Correlations between BOLD signal changes during word processing in the MPFC and DLPFC

\begin{tabular}{|c|c|c|c|c|c|c|c|}
\hline & 1 & 2 & 3 & 4 & 5 & 6 & 7 \\
\hline \multicolumn{8}{|l|}{ 1. MPFC neutral } \\
\hline 2. MPFC positive & $.83^{* * *}$ & & & & & & \\
\hline 3. MPFC negative & $.77^{* * *}$ & $.86^{* * *}$ & & & & & \\
\hline 4. MPFC negative depression & $.84^{* * *}$ & $.85^{* * *}$ & $.85^{* * *}$ & & & & \\
\hline 5. DLPFC neutral & $.50 * *$ & .33 & .32 & .21 & & & \\
\hline 6. DLPFC positive & $.48^{* *}$ & $.38^{*}$ & .31 & .25 & $.88^{* * *}$ & & \\
\hline 7. DLPFC negative & $.37^{*}$ & .19 & .27 & .14 & $.83^{* * *}$ & $.76^{* * *}$ & \\
\hline 8. DLPFC negative depression & $.40^{*}$ & .30 & .32 & .22 & $.75^{* * *}$ & $.84^{* * *}$ & $.71^{* * *}$ \\
\hline
\end{tabular}

Note. MPFC = medial prefrontal cortex DLPFC $=$ dorsolateral prefrontal cortex; ${ }^{* * *}<.001 ;^{* *}<.01 ;^{*}<.05$ 



\section{CHAPTER 3 \\ Sad mood induction modulates default mode network connectivity in chronic depression}

Submitted as:

Renner, F., Siep, N., Arntz, A., van de Ven, V., Peeters, F., Quaedflieg, C., \& Huibers, M. (2014). Sad mood induction modulates default mode network resting-state functional connectivity in chronic depression. 


\begin{abstract}
Background: Major depressive disorder (MDD) is associated with increased default mode network (DMN) resting-state connectivity. The aim of this study was to investigate the effects of a sad mood induction on the DMN resting state connectivity in patients with a current chronic major depressive disorder (cMDD) and matched non-patient controls.
\end{abstract}

Methods: Eighteen cMDD patients and 18 matched non-patient controls underwent a 6 min functional magnetic resonance imaging (fMRI) scan while resting quietly, before and after a sad mood induction consisting of mood suggestive music combined with autobiographical recall. A two-level seed-based functional connectivity analysis was used to assess posterior cingulate cortex (PCC) based DMN functional connectivity of the two resting-state measurements.

Results: The mood induction procedure had a significant effect on self-reported mood ratings in both cMDD and in non-patient controls. PCC connectivity with the parahippocampal gyrus, the superior temporal gyrus and the anterior inferior temporal cortex increased in cMDD patients following the sad mood induction, whereas it decreased in non-patient controls. PCC connectivity with the anterior prefrontal cortex and the precuneus decreased in cMDD patients following the sad mood induction, whereas it increased in non-patient controls.

Conclusions: Present findings are in line with neurobiological models of depression of increased connectivity of the DMN in cMDD patients. It is suggested that the changes in DMN connectivity following the sad mood induction reflect the activation of latent negative-schemas and/or ruminative processes and a failure to exert cognitive control over negative memory retrieval in patients with cMDD.

Keywords: chronic major depressive disorder, resting-state, functional connectivity, default mode network, posterior cingulate cortex 


\section{Introduction}

Major depressive disorder (MDD) is a relatively common, mental disorder that often takes a chronic course (1). The default mode network (DMN), a brain network which has well-documented associations with self-referential processing (2), rumination (3) and autobiographic memory (4), plays a key role in the neuropathology of MDD (5). Numerous studies have now demonstrated that patients with MDD show increased DMN resting-state functional connectivity, compared to non-patient controls (6).

Although the finding of increased connectivity in the DMN of MDD patients seems to be fairly consistent, connectivity within this network might not necessarily be stable. Indeed, studies now show that DMN connectivity is modulated by the mood-state of participants $(7,8)$. Harrison et al. used a mood induction procedure which required healthy participants to recall sad and neutral autobiographical memories during fMRI (7). They found a decrease in functional connectivity within the DMN between the neutral and the sad mood induction recall condition. Zamoscik et al. (2014) studied the effect of negative autobiographical life events recall on connectivity between the Posterior Cingulate Cortex (PCC) and the parahippocampal gryus (PHG), which are two DMN nodes implicated in autobiographical memory. Results showed that remitted depressed participants had stronger connectivity between the PCC and the PHG during the recollection of negative life events compared to non-patient controls (8). These findings support the idea that DMN connectivity in healthy and previously depressed participants is not stable, but rather dynamic, and that it is influenced by changes in emotional states such as sad mood.

The present resting state fMRI study advances previous research on modulation of DMN connectivity in three ways. First, whereas previous studies reveal important mechanisms as to how DMN connectivity can be modulated by a sad mood challenge in healthy or previously depressed individuals, it is unclear how patients with a current MDD modulate connectivity in the DMN following a sad mood challenge. To the best of our knowledge no study to date has tested the effects of a sad mood induction on resting state DMN connectivity in currently depressed individuals. Second, given that connectivity within the DMN is positively correlated with depressive chronicity (9), it is intriguing to study connectivity in patients with chronic major depressive disorder (cMDD). Third, in previous studies DMN connectivity was assessed during the mood induction but it is not clear how resting state DMN connectivity is influenced after sad mood has been triggered.

The aim of the present study was to investigate resting state connectivity of the DMN in patients with a current cMDD and matched non-patient controls before and after a sad mood induction. Based on previous findings it is hypothesized that compared to non-patient controls, cMDD patients would show increased connectivity in the DMN following a sad mood induction. Demonstrating modulation of connectivity in the DMN by a sad mood challenge in patients with current MDD is clinically important, because increased connectivity in the DMN following a mood challenge might reflect ruminative processes or activation of latent maladaptive schemas, defined as memory structures 
that influence cognitions, emotions and behavior, which are primary treatment targets in psychotherapy for depression.

\section{Methods and Materials}

\section{Participants}

Eighteen patients with a chronic major depressive disorder (cMDD) and 18 age, gender, education level, and left/right handedness matched non-patient control participants without any current axis-I disorder participated in this study. One left handed patient could not be matched with a left handed control participant and was therefore matched with a right handed control participant. Patients were recruited from a specialized secondary care outpatient unit in the Netherlands (Riagg Maastricht) as part of a treatment study testing the effects of long term psychotherapy (schema therapy) for cMDD (Clinicaltrials.gov identifier: NCT01153867). All patients were scanned before the start of the intervention phase of the treatment study.

Participants were excluded if they had MRI contraindications. Study inclusion criteria for patients were a current diagnosis of cMDD according to the diagnostic criteria of the Diagnostic and Statistical Manual of Mental Disorders fourth edition (DSM-IV), assessed with the structured clinical interview for axis-I disorders (SCID-I) by trained interviewers and a Beck Depression Inventory second edition (BDI-II) score of 20 or higher at study entry. Exclusion criteria for patients were the presence of bipolar disorder, psychotic disorders, alcohol or substance dependence, or autism spectrum disorders. Patients meeting criteria for a DSM-IV cluster-A or cluster-B personality disorder as assessed with the SCID-II interview were also excluded. One patient with comorbid borderline personality disorder was included in error but is analyzed here. Patients with acute suicide risk were excluded. Use of antidepressant medication at study entry was an exclusion criterion, unless patients were stable on medications for three month or longer prior to study entry. Ten patients $(55.6 \%)$ took antidepressant medication at least 3 month prior to study entry.

Participants in the non-patient control condition were recruited via poster advertisements and a database of volunteer study participants. Control participants were excluded if they met the DSM-IV criteria of any current axis-I disorder or if they met the criteria for a lifetime mood disorder, as assessed with the SCID-I interview by a trained interviewer.

The study protocol was approved by the medical ethical committee of the academic community hospital Maastricht. All participants enrolled in the study received verbal and written information about the purpose and procedure of the study and signed written informed consent.

Demographic and clinical characteristics of the sample are summarized in Table 1. The two groups did not differ statistically significantly on any of the demographic variables. The mean BDI-II score of patients was 30.50 (SD = 9.39), indicating severe depression. The average BDI-II score of non-patient controls was 2.67 (SD = 2.77). Seven 
(38.9\%) of the patients had a comorbid axis-I disorder and 14 (77.8\%) had a comorbid personality disorder. The mean number of previous depressive episodes was 3.38 (SD = 2.39; Median = 3.5).

Table 1. Demographic and clinical characteristics of the sample.

\begin{tabular}{lllll}
\hline & $\begin{array}{l}\text { cMDD } \\
(N=18)\end{array}$ & $\begin{array}{l}\text { Controls } \\
(N=18)\end{array}$ & $\begin{array}{l}t \text {-test } \\
(p \text {-value })\end{array}$ & $\begin{array}{l}\chi 2 \\
(p \text {-value })\end{array}$ \\
\hline $\begin{array}{l}\text { Age, M (SD) } \\
\text { Gender, n (\%) } \\
\quad \text { female } \\
\text { male }\end{array}$ & $41.17(17.53)$ & $42.67(18.73)$ & .81 & - \\
$\begin{array}{l}\text { Education level, n (\%) } \\
\quad \text { low }\end{array}$ & $13(72.2)$ & $13(72.2)$ & - & 1.00 \\
$\quad \begin{array}{l}\text { medium } \\
\text { high }\end{array}$ & $1(5.6)$ & $5(27.8)$ & & \\
BDI-II score & $6(33.3)$ & $2(11.1)$ & - & .68 \\
\hline
\end{tabular}

Note. BDI-II = Beck Depression Inventory second edition.

\section{Procedure}

This study used a mixed design with group (non-patient control, cMDD) as betweensubject factor and two fMRI runs (pre-mood induction, post-mood induction) as within subject factor. The procedure of this study is summarized in Figure 1. First, participants underwent a resting-state scan, which lasted $6 \mathrm{~min}$. Directly after the first resting-state scan, participants were asked to rate their current mood along four visual analogue scales (VAS) using an MR compatible joystick. The four VAS scales measured mood on a bipolar continuum between negative and positive, dull and glad, secure and anxious, and sad and happy. After the initial mood ratings, participants received the instructions for the mood induction and the volume of the music that was used in the mood induction protocol (see below) was adjusted to a level that participants could hear the music loud and clear.

The mood induction consisted of mood suggestive music in combination with autobiographical recall of a personal sad event (8 $\mathrm{min}$ ) in order to induce a sad moodstate. The participants were instructed to recall a personal sad event from their own life while listening to "Adagio for Strings" by Samuel Barber for 8 min via headphones. Mood suggestive music in combination with autobiographical recall reliably induces short lasting sad mood-states (10) and the specific piece of music has successfully been used in our previous studies $(11,12)$. After the music was started the anatomical scan was started and the music volume was increased by approximately $10 \%$ to account for the noise of the scanner. Directly after the mood induction, participants again indicated their current mood-state on the four VAS scales. Following this the second resting-state fMRI scan was started which again lasted 6 minutes. After the second resting-state scan participants again indicated their current mood-state on the four VAS scales. Following 
this part of the experiment, participants engaged in a self-referential processing task and received a diffusion tensor imaging Scan (results not reported here).

Directly after the scanning session participants were asked to indicate whether they had difficulties to immerse in a sad mood-state due to the noise of the scanner along a $100 \mathrm{~mm}$ VAS scale ranging from $0=$ not at all to $100=$ very much.

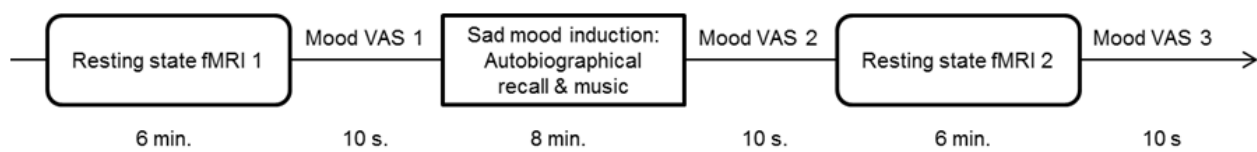

Figure 1. Overview of the design of the current study. The sad mood induction occurred between the two resting-state scans, during structural imaging.

\section{Measures}

\section{Beck Depression Inventory}

Depressive symptom severity was assessed with the Beck Depression Inventory second edition (BDI-II), a 21-item self-report instrument (13). In the present study the Dutch version of the BDI-II was used which has been shown to have high internal consistency in a Dutch sample of psychiatric patients (Cronbach's $\alpha=.92$ ) and adequate construct validity with other depression scales (rs between .79 and.85; 14).

\section{Image acquisition}

Anatomical images were acquired using a Magnetom Allegra 3T scanner (Siemens Healthcare, Netherlands) located at the Faculty of Psychology and Neuroscience, Maastricht University with a T1-weighted gradient echo (196 slices, TR $=2250 \mathrm{~ms}, \mathrm{TE}=$ $26 \mathrm{~ms}$, flip angle $=90^{\circ}$, field of view $=256 \mathrm{~mm}$ and voxel dimensions $1 \times 1 \times 1 \mathrm{~mm}^{3}$ ). A T2*-weighted functional measurement was acquired using a standard echo-planar imaging (EPI) sequence (TR $2000 \mathrm{~ms}$, TE $30 \mathrm{~ms}$, flip angle $=90^{\circ}$, 32 slices, 180 volumes, $3 \times 3 \times 3 \mathrm{~mm}^{3}$ ). A slice tilt correction of -30 was used to minimize inhomogeneity artefacts (15).

\section{fMRI data preprocessing}

Data pre-processing and analyses were conducted with Brainvoyager QX v2.4 (Brain Innovations, Maastricht, the Netherlands). The first two volumes of the functional images were omitted due to magnetization artifacts. Preprocessing of the remaining functional data included slice time correction using sinc interpolation, 3D motion correction using sinc interpolation, spatial smoothing (Gaussian kernel with full-widthat-half-maximum of $4 \mathrm{~mm}$ ) and linear trend removal. Individual functional datasets were then co-registered with structural images of the same participant and 
subsequently normalized using the Talairach and Tournoux transformation procedure (16). Talairach coordinates were subsequently transformed to Montreal Neurological Institute (MNI) coordinates based on (; all coordinates reported in this article are MNI coordinates17). Individual anatomical datasets were then averaged to create a groupbased anatomical brain mask to exclude voxels belonging to the outside of the brain.

\section{Resting-state functional connectivity analysis}

In order to determine change in DMN connectivity following the sad mood induction, we conducted a seed based connectivity analysis. The PCC was selected as seed because this region has been shown to reveal connectivity in the DMN most consistently (18). The anatomical location of the PCC seed was based on the results of a separate selfreferential processing task, performed in the same experimental session by the same participants, following the resting-state scans (19). In this task a whole-brain Random Effects (RFX) ANOVA analyses was used to contrast personality trait words with nonwords. This analysis resulted in the functional localization of the cortical midline structures involved in self-referential processing (20), including the PCC. The peak voxel of the left PCC (5 mm sphere around MNI: -9, -56, 25; Figure 2) was selected as seed, which is comparable in location to regions that previous studies have used to reveal the DMN (e.g. 8, 21).

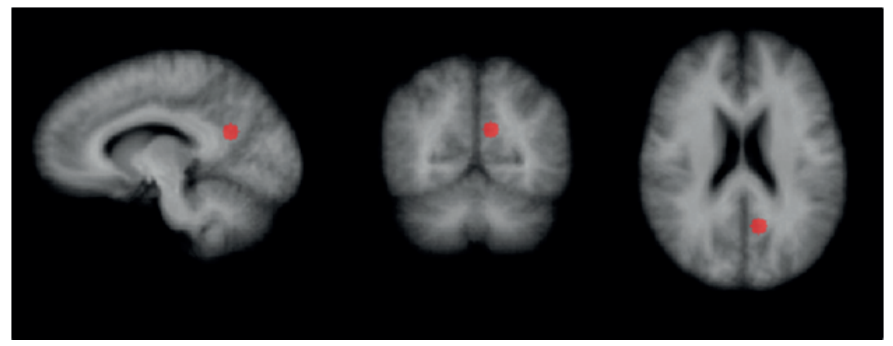

Figure 2. PCC seed selected for the current analyses $(5 \mathrm{~mm}$ sphere around MNI: $-9,-56,25)$ based on the peak voxel of a separate self-referential processing task performed by the same participants in the same session, after the resting-state scans.

To perform the group seed based analysis in Brainvoyager QX, single study design matrix (SDM) files were created for each run of each participant using the NeuroElf MATLAB (Mathworks, Inc.) toolbox (www.neuroelf.net) and a resting state analysis toolbox developed at Maastricht University (www.vincentvandeven.weebly.com/software.html). These SDM files included the BOLD time series of the PCC seed region and $122 \mathrm{z}$-normalized nuisance confound regressors: six 3D head motion parameters and their first derivatives, mean signal from the ventricles and white matter, global signal, and signal oscillations at a frequency above $0.1 \mathrm{~Hz}$ (sine-cosine pairs). The resulting SDM files were then used to run an RFX GLM to find voxels that showed correlation with the PCC seed for each run of each participant. The Pearson correlation coefficients $(r)$ maps were then transformed using Fisher's r-to-z transformation ${ }^{3}$, to yield variates that are approximately normally distributed. The resulting $F$-map showed

${ }^{3} \mathrm{z}=0.5 \operatorname{Ln}[(1+\mathrm{r}) /(1-\mathrm{r})]$ 
all voxels that had a significant correlation with the PCC seed at a conservative false discovery rate (FDR) of 0.001 for all participants over all runs. This map was transformed into a DMN mask (Figure 3), which was used to limit the final main effects and groups x mood induction analyses to the hypothesized DMN network.

In the final analysis, first, the main effect of group on the first resting-state scan (pre-mood induction) and the main effect of run (resting-state pre/post-mood induction) was computed to replicate earlier findings of increased DMN connectivity in depressed patients and to determine the effect of the mood induction on DMN connectivity in general, respectively. Second, a repeated measures ANOVA analysis with group (nonpatient control vs. cMDD) as between subject factor and mood induction (pre/post) as within-subject factor was conducted to test for the hypothesized difference in the effects of the mood induction between the two groups. The resulting three $F$-maps were thresholded at a $p$-value of 0.01 and a cluster size threshold of $135 \mathrm{~mm}^{3}$ (5 voxels) as determined by the cluster-level statistical threshold estimator plugin (BrainVoyager QX; 1000 Monte Carlo simulations).

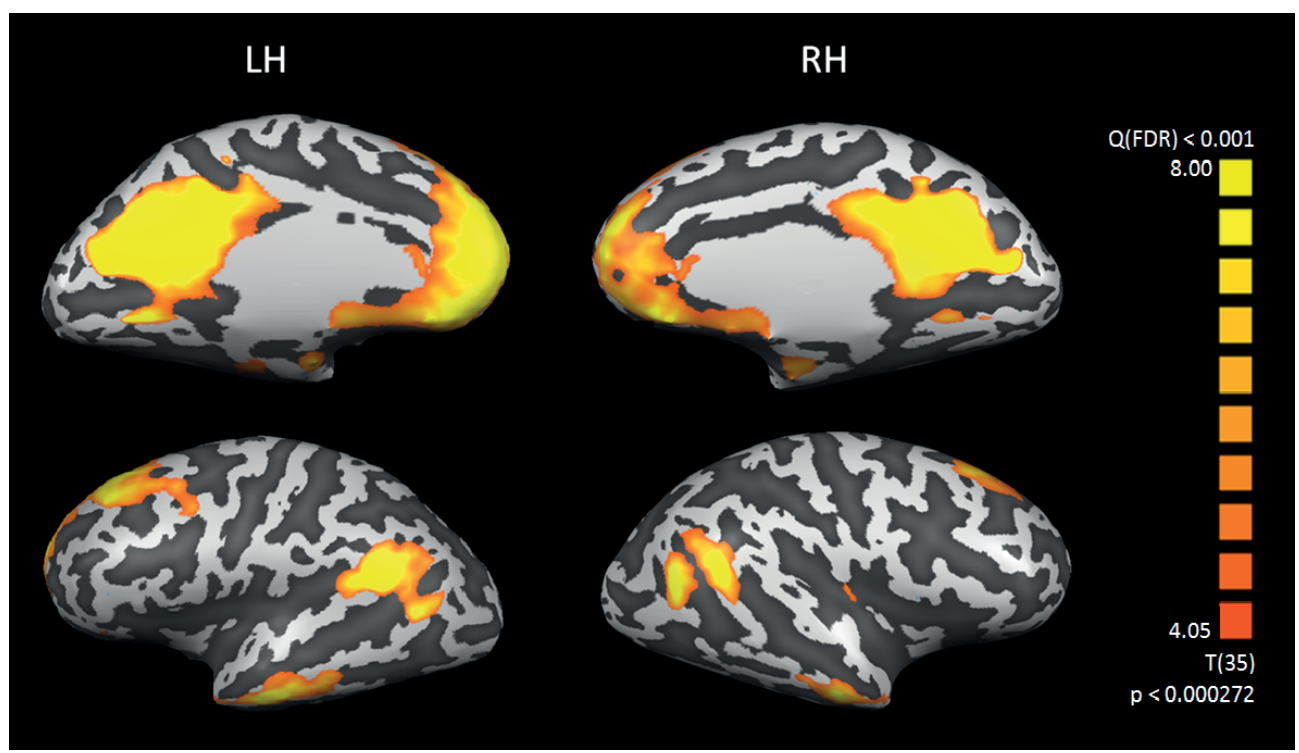

Figure 3. PCC based functional connectivity mask resembling the default mode network.

\section{Results}

\section{Behavioral Data}

\section{Post session questionnaire}

On average participants reported some difficulties to immerse in a sad mood-state due to the noise of the scanner $(M=44.44, S D=32.63)$ on a VAS scale ranging from 0 to 100 , with higher scores indicating more difficulties. Importantly, there were no differences in 
self-reported difficulties to immerse in a sad mood-state due to the noise of the scanner between the two groups, $t(34)=-0.57, p=.56$.

\section{Manipulation check}

Prior to and directly after the mood induction and following the second resting-state scan, participants indicated their current mood-state on four VAS scales ranging from 0 to 100 with higher scores indicating more positive mood-states. To reduce statistical testing, an average mood scale of all four VAS scales was constructed per mood assessment moment. These average mood VAS scales had a high internal consistency, Cronbach's alpha $=.91, .88$, and .85 . Figure 4 is an overview of the repeated mood ratings per group. First we tested whether the mood induction had an effect on the current mood-state of the overall sample. For the overall sample, mood ratings prior to the mood induction $(M=65.02, S D=21.97)$ were significantly higher than following the mood induction $(M=50.06, S D=19.46 ; t(35)=6.25, p<.001)$. Within group comparisons revealed a significant decrease in current mood-state for both the nonpatient control participants and cMDD patients, $(t(17)=6.04, p<.001$ and $t(17)=3.18$, $p=.005$, respectively). The decrease in current mood-state from pre-mood induction to post-mood induction was slightly stronger in non-patient control participants, but the interaction effect did not reach the conventional level of statistical significance $F(1,34)=$ $3.87, p=.057$. The mood-state at the end of the experiment remained at a lower level compared to pre-mood induction assessment for both patients $(t(17)=2.76, p=.01)$ and non-patient controls $(t(17)=3.46, p<.01)$. At each time point there was a significant main effect of group, showing that patients reported a lower mood-state at pre-mood induction, $t(34)=-7.58, p<.001$, at post-mood induction, $t(34)=-5.11, p<.001$, and after the second resting-state scan, $t(32)=-5.29, p<.001$.

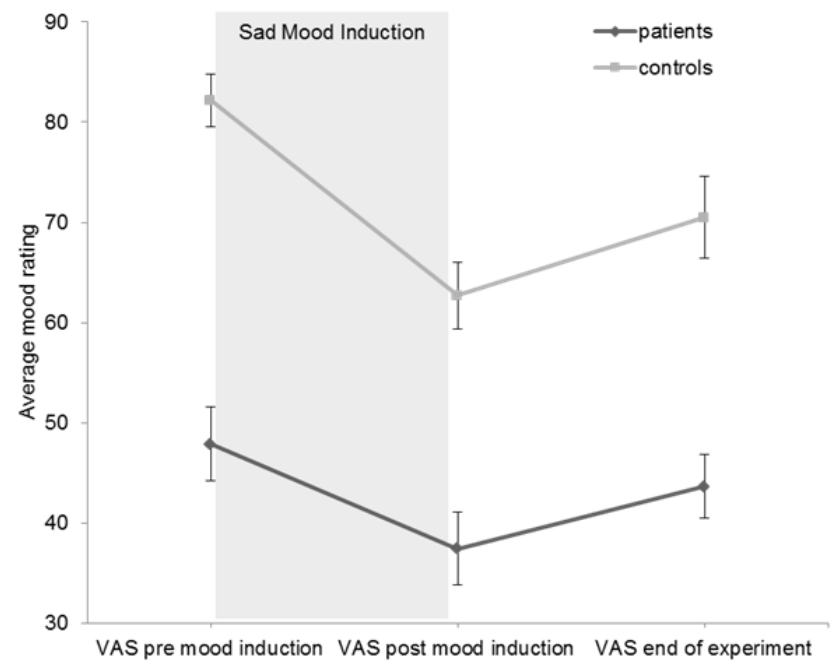

Figure 4. Mood ratings prior to the mood induction (T1), after the mood induction (T2) and at the end of the experiment (T3); higher scores represent more positive mood 
fMRI results resting-state functional connectivity

\section{Main effect group}

Prior to the mood induction and in line with previous findings, cMDD patients showed greater connectivity between the PCC and the medial temporal gyrus $(52,-68,28)$, the dorsolateral medial prefrontal cortex $(10,61,30)$, the precuneus $(-6,-65,29)$, the anterior prefrontal cortex $(-6,57,30)$, the PCC $(-6,56,10)$, the superior occipital gyrus $(-45,-78,34)$ and the medial temporal gyrus $(-58,-59,15)$ compared to the non-patient controls prior to the mood induction.

\section{Main effect pre- to post-mood induction}

Indicative of the success of the experimental manipulation, there was an overall significant decrease in connectivity from pre-mood induction to post-mood induction in the precuneus $(-6,-58,35)$, and in two separate clusters of the parahippocampal gyrus ($13,-33,-1$ and $-36,-25,-18)$.

\section{Group on mood induction interaction}

Five ROIs with significant group (cMDD/non-patient controls) $\times$ mood induction (pre/post) interactions were identified (Figure 5; Table 2), including the anterior inferior temporal cortex, the superior temporal gyrus, the uncus / anterior parahippocampal gyrus, the anterior prefrontal cortex, and the precuneus. In line with our hypothesis the anterior inferior temporal cortex, the superior temporal gyrus and the uncus / anterior parahippocampal gyrus showed increased connectivity after the mood induction in the cMDD patients compared to the non-patient controls. However, the precuneus and anterior prefrontal cortex showed the opposite pattern, with increased connectivity after the mood induction for the non-patient controls compared to the patients.

\section{Association of PCC connectivity with subjective measures}

Depressive symptom severity and mood reactivity was correlated with change in connectivity from pre to post-mood induction across groups (Table 3). Depressive symptom severity correlated negatively with change in connectivity between the PCC and the anterior prefrontal cortex and the precuneus and positively with the remaining areas. Mood reactivity correlated positively with change in connectivity between PCC and the anterior prefrontal cortex and the precuneus, showing that more negative mood ratings following the sad mood induction were associated with a stronger increase in connectivity in these areas. 


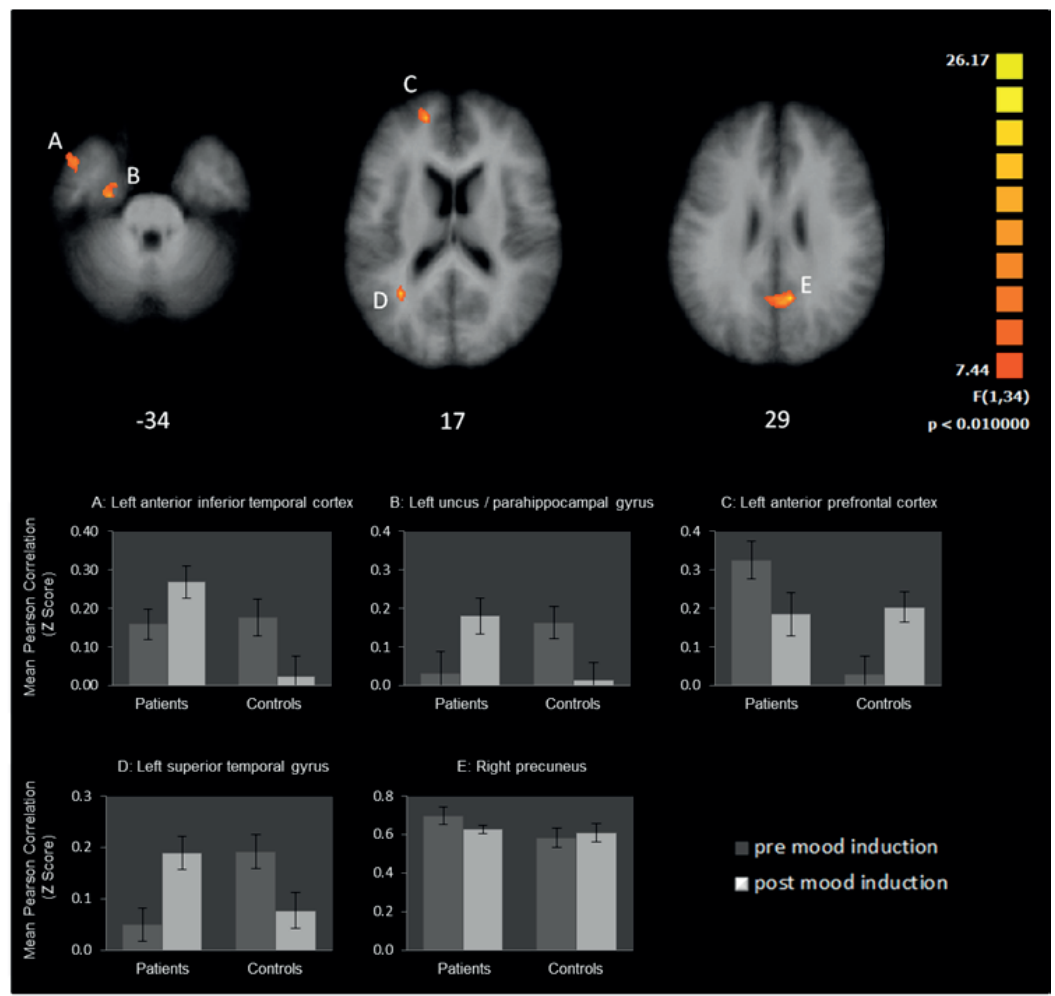

Figure 5. Significant group (cMDD/non-patient controls) $\times$ mood induction (pre/post) interactions. The $\mathrm{y}$-axis of the graphs depicts the mean $\mathrm{z}$ values of functional connectivity of the PCC with the DMN.

Table 2. Region of interest details identified for the group (cMDD/non-patient controls) $\times$ mood induction (pre/post) interactions.

\begin{tabular}{|c|c|c|c|c|c|c|c|c|c|}
\hline \multirow{2}{*}{\multicolumn{2}{|c|}{ Brain region }} & \multirow[b]{2}{*}{ L/R MNI $(\mathrm{x}, \mathrm{y}, \mathrm{z})$} & \multirow[b]{2}{*}{$\begin{array}{l}\text { Cluster } \\
\text { size } \\
\text { Voxels }\end{array}$} & \multirow[b]{2}{*}{$\mathrm{F}$} & \multirow[b]{2}{*}{$p$-value } & \multicolumn{2}{|c|}{$\begin{array}{l}\text { Average } \\
\text { connectivity } \\
\text { change Fisher's Z }\end{array}$} & \multicolumn{2}{|c|}{$\begin{array}{l}\text { Cohen's d } \\
\text { (change within } \\
\text { groups) }\end{array}$} \\
\hline & & & & & & cMDD & controls & cMDD & controls \\
\hline $\begin{array}{l}\text { Anterior inferior } \\
\text { temporal cortex }\end{array}$ & $\mathrm{L}$ & $-49,9,-34$ & 262 & 13.83 & $<.001$ & 0.11 & -0.15 & 0.37 & -0.46 \\
\hline $\begin{array}{l}\text { Uncus / parahippocampal } \\
\text { gyrus }\end{array}$ & & $-26,-11,-28$ & 178 & 18.96 & $<.001$ & 0.15 & -0.15 & 0.45 & -0.49 \\
\hline $\begin{array}{l}\text { Anterior prefrontal } \\
\text { cortex }\end{array}$ & $\mathrm{L}$ & $-16,52,18$ & 633 & 27.61 & $<.001$ & -0.14 & 0.18 & -0.42 & 0.57 \\
\hline Superior temporal gyrus & $\mathrm{L}$ & $-32,-53,21$ & 184 & 28.98 & $<.001$ & 0.14 & -0.11 & 0.53 & -0.43 \\
\hline Precuneus & $\mathrm{R}$ & $10,-52,31$ & 615 & 4.44 & .043 & -0.07 & 0.03 & -0.25 & 0.09 \\
\hline
\end{tabular}

Note. $\mathrm{L} / \mathrm{R}=$ Left $/$ Right; Cohen's d = Mean difference/pooled SD. 
Table 3. Correlations between change in PCC functional connectivity, depressive symptom severity, and mood reactivity

\begin{tabular}{lll}
\hline PCC connectivity with & BDI-II & Mood reactivity \\
\hline Anterior inferior temporal cortex & $.52^{* *}$ & -.01 \\
Uncus / parahippocampal gyrus & $.57^{* *}$ & -.12 \\
Anterior prefrontal cortex & $-.53^{* * *}$ & $.37^{*}$ \\
Superior temporal gyrus & $.63^{* * *}$ & -.07 \\
Precuneus & $-.36^{*}$ & $.48^{* *}$ \\
\hline
\end{tabular}

Note. ${ }^{*} p<.05 ;{ }^{* *} p<.01 ;{ }^{* * *} p<.01$; BDI-II = Beck Depression Inventory second edition; Mood reactivity = Average mood ratings following the mood induction - average mood ratings before the mood induction, with positive scores indicating stronger mood reactivity.

\section{Discussion}

The aim of the current fMRI study was to investigate resting state connectivity of the DMN in patients with a current cMDD and matched non-patient controls before and after a sad mood induction. The mood induction consisted of mood suggestive music in combination with autobiographical recall and resulted in increased negative mood ratings in both cMDD patients and non-patient controls. Using a PCC seed, main effect analyses of group prior to the mood induction replicated previous findings showing increased DMN connectivity in patients with depression (for a review see: 6). Importantly, main effect analyses of mood induction showed that DMN connectivity, particular in the precuneus and parahippocampal gyrus, is modulated by the current mood-state of participants.

The main focus of the present study was on the hypothesized increase in DMN connectivity following the sad mood induction in cMDD patients, possibly reflecting an increased ruminative self-focus $(3,22)$ or activation of latent dysfunctional schemas. In line with this hypothesis, results showed increased connectivity with the PCC following the sad mood induction in cMDD patients in the anterior inferior temporal cortex, the superior temporal gyrus and the parahippocampal gyrus. Following the sad mood induction, the precuneus showed a slight decrease in connectivity with the PCC in cMDD patients and the anterior prefrontal cortex showed increased connectivity with the PCC in non-patient controls. This suggests that specifically temporal areas and the parahippocampal gyrus are associated with increased PCC connectivity in cMDD patients, following the sad mood induction, whereas other DMN regions show an opposite pattern.

The increase in DMN connectivity in CMDD patients in the present and previous studies might be interpreted as activation of latent dysfunctional schemas. Dysfunctional schemas can be conceptualized as latent memory structures that have coded past experiences and when activated by a triggering event influence cognitions, emotions and 
behavior (e.g. 23). It has been shown previously, that negative memory retrieval differentially activates the temporal gyrus, when contrasted with positive memory retrieval (24). In line with these findings, it has been argued, that the ease with which dysfunctional schemas in depression are activated by external triggers is related to increased task negative connectivity with, among other brain regions, the (para)hippocampus (5). In general, the present finding of increased DMN connectivity, following the sad mood induction in cMDD patients supports neurobiological models of ruminative thoughts in depression, proposing that increased parahippocampal activity is involved in ruminative thoughts (25). Importantly, in the current study the mood induction procedure was not conducted during but before functional imaging of the second resting-state scan. The increased connectivity following the mood induction in patients vs. controls therefore likely reflects a relatively natural reaction of patients, after being challenged with sad mood, rather than compliance of the task instruction to recall sad autobiographical events or processing of external stimuli.

The finding of the current study of decreased DMN connectivity in non-patient controls, following the sad mood induction are in line with those of a previous fMRI study that reported decreased functional connectivity of the DMN during recall of sad autobiographical events combined with mood suggestive music in healthy participants (7). These findings suggest that a sad mood induction in healthy participants results in more negative mood as reflected in self-report data, but not in the activation of negative latent schemas or dysfunctional cognitive processing as indicated by the neuroimaging data. In line with this finding, a recent study found increased DMN connectivity in remitted depressed patients, compared to healthy controls, following a sad mood induction (8). Together, results from these two previous studies that used a mood challenge and the present study suggest that the DMN network can be modulated by subjective mood-states and that the increased connectivity in remitted (8) and currently depressed participants likely reflects activation of latent dysfunctional schemas and/or increased rumination about negative issues. Non-fMRI studies have shown that remitted depressed patients cannot be distinguished from healthy participants on measures of dysfunctional cognitions or schemas (for a review see: 26). However, when challenged with a mood induction, remitted depressed patients activate latent negative schemas (26) and the strength of this cognitive reactivity might predict subsequent relapse (27).

PCC - anterior prefrontal cortex connectivity was increased in non-patients controls and decreased in cMDD patients following the sad mood induction. Anterior prefrontal cortex subdivisions have previously been associated with cognitive control of memory retrieval (e.g. 28, 29). It is possible that the increased connectivity following the sad mood induction in non-patients controls in the current study reflects an attempt to control retrieval of negative memories. In contrast, patients with cMDD might fail to control retrieval of negative memories following the sad mood induction. This argumentation is in line with non-fMRI studies suggesting that depression is associated with impairments in cognitive control (for a review see: 30) and with theoretical models proposing that reduced prefrontal control in combination with activation of negative schemas in depression gives rise to a downward spiral of rumination and negative affect 
(31). Interestingly, in the current study PCC connectivity with the anterior prefrontal cortex positively correlated with mood reactivity across participants. Thus, the stronger the emotional trigger the more readily cognitive control over memory retrieval might be recruited. Clinically, these findings suggest that patients with cMDD fail to control or stop negative memory retrieval once they are in a sad mood-state. In contrast, nondepressed individuals control memory retrieval during sad mood possibly reflecting a coping strategy that prevents healthy individuals from entering a negative downward spiral of dysfunctional cognitive processing and negative affect.

A limitation of this study is that it did not include a clinical control group and therefore the specificity of the present findings with regard to chronic depression remains unclear. It is possible, for example, that patients with non-chronic depression show comparable connectivity following the sad mood induction. Moreover, about half of the patients participating in this study took antidepressant medication at the time when the study was conducted and it is unclear if this has influenced the results.

In conclusion, the present study suggests that DMN connectivity increases in cMDD patients but not in controls following a sad mood induction. This finding has important clinical implications, as the increased DMN connectivity triggered by sad mood might reflect the activation of latent negative schemas and/or ruminative processing in depression. Patients with cMDD might fail to exert cognitive control over memory retrieval processes once sad mood is triggered whereas non-patients controls seem to increase cognitive control processes possibly in an attempt to cope with the sad moodstate. This coping strategy prevents non-depressed individuals from entering a negative downward spiral of dysfunctional cognitive processing and negative affect. Increasing cognitive control in patients with depression either through neurostimulation (e.g. 32) or through behavioral interventions might be a fruitful avenue in the treatment of cMDD. Future research should determine if treatment for cMDD that specifically focusses on decreasing the impact of these negative schemas (e.g. schema therapy; 33) has an impact on DMN connectivity following a sad mood induction, in patients with cMDD.

\section{Acknowledgments}

We are grateful to our colleagues at Maastricht University who supported this study: Annie Hendriks and Annie Raven helped with patient recruitment; Danielle Jongen assisted with recruitment of matched controls. Thanks are also due to our colleagues at the Riagg Maastricht who helped to recruit patients and conducted clinical interviews for this study.

\section{Financial Disclosures}

None. 


\section{References}

1. Richards D (2011): Prevalence and clinical course of depression: a review. Clin Psychol Rev. 31:1117-1125.

2. Whitfield-Gabrieli S, Moran JM, Nieto-Castanon A, Triantafyllou C, Saxe R, Gabrieli JD (2011): Associations and dissociations between default and self-reference networks in the human brain. NeuroImage. 55:225-232.

3. Berman MG, Peltier S, Nee DE, Kross E, Deldin PJ, Jonides J (2011): Depression, rumination and the default network. Soc Cogn Affect Neurosci. 6:548-555.

4. Spreng RN, Mar RA, Kim AS (2009): The common neural basis of autobiographical memory, prospection, navigation, theory of mind, and the default mode: a quantitative meta-analysis. J Cogn Neurosci. 21:489-510.

5. Marchetti I, Koster EH, Sonuga-Barke EJ, De Raedt R (2012): The default mode network and recurrent depression: a neurobiological model of cognitive risk factors. Neuropsychol Rev. 22:229251.

6. Whitfield-Gabrieli S, Ford JM (2012): Default mode network activity and connectivity in psychopathology. Annu Rev Clin Psychol. 8:49-76.

7. Harrison BJ, Pujol J, Ortiz H, Fornito A, Pantelis C, Y $\tilde{A}^{1} / 4$ cel M (2008): Modulation of Brain RestingState Networks by Sad Mood Induction. PLoS ONE. 3:e1794.

8. Zamoscik V, Huffziger S, Ebner-Priemer U, Kuehner C, Kirsch P (2014): Increased involvement of the parahippocampal gyri in a sad mood predicts future depressive symptoms. Soc Cogn Affect Neurosci.

9. Greicius MD, Flores BH, Menon V, Glover GH, Solvason HB, Kenna H, et al. (2007): Resting-State Functional Connectivity in Major Depression: Abnormally Increased Contributions from Subgenual Cingulate Cortex and Thalamus. Biol Psychiatry. 62:429-437.

10. Martin M (1990): On the induction of mood. Clin Psychol Rev. 10:669-697.

11. Renner F, Schwarz P, Peters ML, Huibers MJH (2014): Effects of a best-possible-self mental imagery exercise on mood and dysfunctional attitudes. Psychiatry Res. 215:105-110.

12. Werthmann J, Renner F, Roefs A, Huibers MJ, Plumanns L, Krott N, et al. (2014): Looking at food in sad mood: do attention biases lead emotional eaters into overeating after a negative mood induction? Eat Behav. 15:230-236.

13. Beck AT, Steer RA, Brown GK (1996): Manual for the Beck Depression Inventory-II. San Antonio, TX.

14. van der Does AJW (2002): Handleiding: De Nederlandse versie van de Beck Depression Inventory, 2de Editie. [The Dutch version of the Beck Depression Inventory, 2nd ed.]. Lisse: Swets and Zeitlinger b.v.

15. Weiskopf N, Hutton C, Josephs O, Deichmann R (2006): Optimal EPI parameters for reduction of susceptibility-induced BOLD sensitivity losses: A whole-brain analysis at $3 \mathrm{~T}$ and $1.5 \mathrm{~T}$. Neuroimage. 33:493-504.

16. Talairach J, Tournoux P (1988): Co-planar stereotaxic atlas of the human brain. New York: Thieme.

17. Lancaster JL, Tordesillas-Gutiérrez D, Martinez M, Salinas F, Evans A, Zilles K, et al. (2007): Bias between MNI and Talairach coordinates analyzed using the ICBM-152 brain template. Hum Brain Mapp. 28:1194-1205.

18. Greicius MD, Krasnow B, Reiss AL, Menon V (2003): Functional connectivity in the resting brain: a network analysis of the default mode hypothesis. Proc Natl Acad Sci U S A. 100:253-258.

19. Renner F, Siep N, Lobbestael J, Arntz A, Peeters F, Huibers M (2014): Neural correlates of selfreferential processing and implicit self-associations in chronic depression. Submitted manuscript.

20. Northoff G, Heinzel A, de Greck M, Bermpohl F, Dobrowolny H, Panksepp J (2006): Self-referential processing in our brain--A meta-analysis of imaging studies on the self. Neuroimage. 31:440-457.

21. Pannekoek JN, van der Werff SJA, Meens PHF, van den Bulk BG, Jolles DD, Veer IM, et al. (2014): Aberrant resting-state functional connectivity in limbic and salience networks in treatment-naïve clinically depressed adolescents. Journal of Child Psychology and Psychiatry.n/a-n/a. 
22. Hamilton JP, Furman DJ, Chang C, Thomason ME, Dennis E, Gotlib IH (2011): Default-mode and task-positive network activity in major depressive disorder: implications for adaptive and maladaptive rumination. Biol Psychiatry. 70:327-333.

23. James IA, Reichelt FK, Freeston MH, Barton SB (2007): Schemas as memories: Implications for treatment. Journal of Cognitive Psychotherapy. 21:51-57.

24. Piefke M, Weiss PH, Zilles K, Markowitsch HJ, Fink GR (2003): Differential remoteness and emotional tone modulate the neural correlates of autobiographical memory. Brain. 126:650-668.

25. Disner SG, Beevers CG, Haigh EAP, Beck AT (2011): Neural mechanisms of the cognitive model of depression. Nat Rev Neurosci. advance online publication.

26. Scher CD, Ingram RE, Segal ZV (2005): Cognitive reactivity and vulnerability: Empirical evaluation of construct activation and cognitive diatheses in unipolar depression. Clin Psychol Rev. 25:487-510.

27. Segal ZV, Kennedy S, Gemar M, Hood K, Pedersen R, Buis T (2006): Cognitive Reactivity to Sad Mood Provocation and the Prediction of Depressive Relapse. Arch Gen Psychiatry. 63:749-755.

28. Barredo J, Öztekin I, Badre D (2013): Ventral Fronto-Temporal Pathway Supporting Cognitive Control of Episodic Memory Retrieval. Cereb Cortex.

29. Badre D, Wagner AD (2007): Left ventrolateral prefrontal cortex and the cognitive control of memory. Neuropsychologia. 45:2883-2901.

30. Gotlib IH, Joormann J (2010): Cognition and depression: current status and future directions. Annu Rev Clin Psychol. 6:285-312.

31. De Raedt R, Koster EW (2010): Understanding vulnerability for depression from a cognitive neuroscience perspective: A reappraisal of attentional factors and a new conceptual framework. Cognitive, Affective, \& Behavioral Neuroscience. 10:50-70.

32. Wolkenstein L, Plewnia C (2013): Amelioration of cognitive control in depression by transcranial direct current stimulation. Biol Psychiatry. 73:646-651.

33. Young JE, Klosko J, Weishaar ME (2003): Schema therapy: A practitioner's guide. New York: Guilford Press. 


\section{PART II}

\section{STABILITY OF UNDERLYING RISK-FACTORS FOR DEPRESSION}





\section{CHAPTER 4 \\ Stability of schemas during treatment for depression}

Published as:

Renner, F., Lobbestael, J., Peeters, F., Arntz, A., \& Huibers, M. (2012). Early maladaptive schemas in depressed patients: Stability and relation with depressive symptoms over the course of treatment. Journal of Affective Disorders, 136(3), 581-590. 


\begin{abstract}
Background: Early maladaptive schemas (EMSs) are hypothesized to be stable, trait-like, enduring beliefs underlying chronic and recurrent psychological disorders. We studied the relation of EMSs with depressive symptom severity and tested the stability of EMSs over a course of evidence-based outpatient treatment for depression in a naturalistic treatment setting.
\end{abstract}

Methods: The sample consisted of depressed outpatients $(N=132)$ treated at a specialized mood disorders treatment unit in the Netherlands. Participants completed measures of depressive symptom severity and maladaptive schemas before treatment and 16-weeks after starting with treatment.

Results: Specific maladaptive schemas (failure, emotional deprivation, abandonment/instability) were cross-sectionally related to depressive symptom severity. Moreover, the schema domain impaired autonomy \& performance at pre-treatment related positively to depression levels at the 16-week follow-up assessment, whereas the schema domain overvigilance \& inhibition at pre-treatment related negatively to depression levels at the follow-up assessment when controlling for pre-treatment depression severity. Finally, all EMSs demonstrated good relative stability over the course of treatment.

Conclusions: Our results suggest that specific EMSs are related to depressive symptom severity in clinically depressed patients, that specific schema domains predict treatment outcome, and that schemas are robust to change over time, even after evidence-based outpatient treatment for depression.

Keywords: Depression; early maladaptive schemas; core beliefs; cognitive vulnerability 


\section{Introduction}

According to the cognitive theory of depression (Beck, 1964) negative beliefs about the self, the world, and the future incorporated in stable cognitive schemas are the key vulnerability factor to depression. In most accounts of cognitive theory, dysfunctional cognitions can best be understood in terms of a hierarchical model of generality with automatic thoughts at the most superficial level, dysfunctional attitudes at an intermediate level, and cognitive schemas at the deepest level (Clark and Beck, 1999; Segal, 1988). While depressogenic cognition have usually been assessed at the level of automatic thoughts or dysfunctional attitudes (Segal and Swallow, 1994), studies assessing dysfunctional cognition at the schema level in depressed patients are sparse. One reason for this might be that schemas are usually considered as implicit cognitive structures that are not readily accessible (Segal, 1988).

Young recently revised the schema concept, emphasizing early maladaptive schemas (EMSs) as key structures in the development of psychopathology (Young, 1995). EMSs are defined as stable, trait-like, enduring beliefs about oneself and the world that are rooted in early childhood experiences (Young et al., 2003). EMSs are in many ways comparable to the cognitive theory concept of core beliefs, defined as the cognitive content of schemas (Clark and Beck, 1999) though there are also important differences between these two concepts (James et al., 2004). For example, core beliefs in depression were usually divided into three broad categories (helplessness, inadequacy, and unloveability), whereas EMSs are more specific. To date, 18 specific EMSs were identified and divided into five broader domains (see Table 1).

Although the concept of EMSs provides a valuable extension to the cognitive theory of depression concepts of automatic thoughts and dysfunctional attitudes, studies relating EMSs to depressive symptoms in depressed patients are relatively sparse. In non-clinically depressed samples it has been shown that the EMSs failure, defectiveness/shame, and self-sacrifice were associated with depressive symptom severity (Calvete et al., 2005). Another study found that the EMSs defectiveness/shame, insufficient self-control, vulnerability, and incompetence/inferiority were crosssectionally related to depressive symptom severity in undergraduate students (Harris and Curtin, 2002). In a mixed clinical sample with mainly depressed patients it was found that the total score on the schema questionnaire (SQ; Young and Brown, 1994) was related to depressive symptom severity, even after controlling for neuroticism and other personality dimensions, suggesting that EMSs explain variance in depressive symptom severity beyond other trait-like constructs that are known to be related to depressive symptoms, like neuroticism (Thimm, 2010). In another clinical sample with mainly depressive symptoms it has been shown that the EMSs abandonment/instability, defectiveness/shame, failure, subjugation, and vulnerability to harm were crosssectionally related to depression severity (Petrocelli et al., 2001). In depressed patients the schema domains undesirability, impaired autonomy \& performance, and impaired limits were cross-sectionally related to depression severity (Halvorsen et al., 2009). Finally, another study found that in depressed outpatients the EMSs 
defectiveness/shame, self-sacrifice, and insufficient self-control were related to depressive symptom severity (Shah and Waller, 2000).

Taken together, studies relating schema domains and specific EMSs to depressive symptom severity found that a wide range of EMSs are related to depressive symptom severity, especially those belonging to the impaired autonomy \& performance and to the disconnection \& rejection domains. While previous research has exclusively focused on the concurrent relation between EMSs and depressive symptoms, the relation of EMSs with symptom improvement during treatment for depression remains unclear.

When studying the relation between EMSs and depressive symptoms in depressed patients, it is important to also determine whether EMSs remain stable in the context of change in depressive symptoms (i.e., during treatment). A fundamental assumption in schema-theory is that EMSs are stable, trait-like constructs that are resistant to change (Young et al., 2003). Accordingly, one would not expect EMSs to change over the course of short-term outpatient treatment that is focused on reducing depressive symptomatology instead of decreasing EMSs. Riso and colleagues (2006) examined the long-term stability of EMSs in 55 depressed outpatients over a course of 2.5 to 5 years and found that EMSs exhibited good stability, comparable with that of personality disorder features (Riso et al., 2006). Similarly, Wang et al., (2010) found moderate stability for most EMSs in depressed patients after 9 years follow-up. While these studies suggest that EMSs exhibit good long term stability in depressed patients, the stability of EMSs over a course of outpatient treatment for depression remains unclear. To the best of the authors' knowledge, the present study is the first to investigate the stability of EMSs in depressed patients over a course of outpatient treatment for depression.

Previous work on the stability of EMSs has emphasized the importance of considering both absolute stability and relative stability when studying change in EMSs over time (Riso et al., 2006). Absolute stability refers to the stability in the mean level of the construct under study over time, whereas relative stability refers to the degree to which relative differences between individuals remain over time (Santor et al., 1997). It has been shown that, in the context of acute change in depressive symptoms (i.e., during depression treatment), dysfunctional cognitions can show large changes in mean level stability in the presence of strong relative stability (Beevers and Miller, 2004; Zuroff et al., 1999). Such findings suggest that measures of dysfunctional cognitions in depressed individuals tap both mood dependent, state-like properties, as evident in changes in mean level stability, as well as mood independent, trait-like properties, as evident in strong relative stability (Beevers and Miller, 2004; Zuroff et al., 1999). Given that EMSs are likely related to depression severity and given that depression severity scores are likely to decrease during treatment. both absolute and relative stability should be considered when studying the stability of EMSs in the context of symptom change. 
Table 1. - A brief description of the five schema domains and all 18 early maladaptive schemas (EMSs), based on Young et al. 2003.

\begin{tabular}{ll}
$\begin{array}{l}\text { Schema domains and early } \\
\text { maladaptive schemas }\end{array}$ & Description \\
\hline $\begin{array}{l}\text { Disconnection \& } \\
\text { Rejection }\end{array}$ & $\begin{array}{l}\text { Schemas that involve expectations that one's needs for security and stability } \\
\text { will not be met in a predictable manner. }\end{array}$ \\
$\begin{array}{l}\text { Mbandonment / Instability } \\
\text { The perceived instability or unreliability of those available for support. } \\
\text { The expectation that others will intentionally hurt, abuse, humiliate, cheat, } \\
\text { lie, manipulate or take advantage. } \\
\text { The expectation that one's desire for emotional support, nurturance, } \\
\text { empathy or protection by others will not be met. }\end{array}$ \\
$\begin{array}{l}\text { Defectiveness / Shame } \\
\text { Social Isolation }\end{array}$ & $\begin{array}{l}\text { The feeling that one is defective, bad, unwanted, inferior, or invalid. } \\
\text { part of a community }\end{array}$
\end{tabular}

Impaired Autonomy \&

Schemas that involve expectations about oneself and the environment that Performance

Dependence /

Incompetence

Vulnerability to Harm or

Illness

Enmeshment interfere with one's perceived ability to function independently and to perform successfully.

The belief that one is not able to handle everyday responsibilities without help from others.

Exaggerated fear that an unpreventable medical, emotional or external catastrophe will strike.

Excessive emotional involvement with significant others at the expense of individualization

Failure

The belief that one has failed or will fail in areas of achievement.

\section{Impaired Limits}

Entitlement

Insufficient Self-Control

\section{Other-Directedness}

Subjugation

Self-Sacrifice

Approval-Seeking

\section{Overvigilance \& Inhibition}

Negativity

Emotional Inhibition

Unrelenting Standards

Punitiveness
Schemas involving a deficiency in internal limits and responsibility to others. The belief that one is superior to others and entitled to special rights and privileges.

A pervasive difficulty or refusal to exercise sufficient self-control and frustration tolerance to achieve personal goals.

Schemas that involve an excessive focus of the desires and feelings of others Surrendering of control to others to avoid negative consequences.

The excessive focus of meting needs of others at the expense of one's own gratification.

An excessive focus on gaining approval, recognition, or attention from others.

Schemas that involve an overemphasis on suppressing one's spontaneous impulses and feelings

A lifelong focus on the negative aspects of life while minimizing the positive aspects.

Inhibiting spontaneous action, feelings, or communication to avoid disapproval by others or feelings of shame.

The belief that one must strive to meet very high standards to avoid criticism. The belief that people should be harshly punished for mistakes. 
In the present study, we sought to further examine the relation between EMSs and depressive symptoms and the stability of EMSs over a course of outpatient treatment for depression in patients diagnosed with Major Depressive Disorder (MDD). We hypothesized that (1) EMSs from the disconnection \& rejection and from the impaired autonomy \& performance domains are cross-sectionally related to depressive symptom severity; (2) high initial levels of EMSs domains are negatively related to the improvement of depressive symptoms over the course of treatment; (3) EMSs remain relatively stable over a course of outpatient treatment for depression.

\section{Method}

\section{Participants}

The present report is based on a sample of 132 depressed outpatients treated at the mood disorder treatment program of the Academic Community Mental Health Center Maastricht (RIAGG Maastricht, The Netherlands). In this secondary care facility, depressed individuals are preferably treated with cognitive therapy (CT), interpersonal therapy (IPT), antidepressant medication (ADM), or a combination of psychotherapy and medication. The inclusion criteria for participation in the present study was a Diagnostic and Statistical Manual of Mental Disorders (DSM-IV; American Psychiatric Association, 1994) diagnosis of MDD as assessed by the Structured Clinical Interview for DSM-IV (SCID-I; First et al., 1996). Participants were excluded if they had a primary diagnosis other than MDD, high acute suicide risk, or insufficient Dutch language skills. The mean age of the sample was 40.7 years ( $S D=12.06$ ); $58 \%$ were female; $33.3 \%$ were married, $25 \%$ were single, $17.4 \%$ cohabited with their partner, $17.4 \%$ were divorced, $3.8 \%$ were widowed, and $3.1 \%$ had a spouse but did not live together. At pre-treatment, the sample was characterized by moderate to severe levels of depressive symptom severity according to the Beck Depression Inventory second edition (BDI-II; Beck et al., 1996), $M=29.42, S D=10.33$. The mean number of previous depressive episodes was 1.82 ( $S D=3.17$ ). Of the overall sample, $42.2 \%$ also had a comorbid Axis-I diagnosis in addition to a primary diagnosis of MDD. Comorbid anxiety disorders were the most common $(31.8 \%)$ followed by substance-related disorders $(10.6 \%)$, eating disorders (7.6\%) and somatoform disorders (3\%). There were no statistically significant differences between patients with comorbid Axis-I diagnosis and patients without comorbid Axis-I diagnosis with respect to BDI-II pre-treatment levels (time 1), $t(130)=-$ $1.43, p=.16$, BDI-II levels at the 16-week follow-up assessment (time 2), $t(83)=-1.28, p$ $=.21$, total SQ score at time $1, t(130)=-1.76, p=.08$, or total SQ score at time $2, t(83)=-$ $0.64, p=.52$.

Of the overall sample of 132 patients that entered the study and completed questionnaires at time 1, 85 patients (64\%) also completed the questionnaires at time 2 . Patients who completed both assessments reported a higher mean age at pre-treatment $(M=43.04, S D=12.24)$, compared to patients who did not provide a time 2 assessment $(M=36.45, S D=10.59), t(130)=3.10, p=.002$. There were no statistical significant 
differences in gender distribution $\chi^{2}(1, N=132)=1.21, p=.27$. Moreover, the two samples did not differ statistically significantly with respect to pre-treatment depressive symptom severity, $t(130)=0.19, p=.85$ or pre-treatment EMSs (all $p$ values $>.003$ ). ${ }^{4}$ Therefore, we considered the sub-sample of completers as representative for the overall sample with respect to symptom severity, EMSs, and gender distribution.

Of the overall sample, 51 patients (38.6\%) received CT, 29 patients $(22 \%)$ received a combination of CT and ADM, 21 patients (15.9\%) received IPT, 12 patients (9.1\%) received a combination of IPT and ADM, 12 patients (9.1\%) received ADM, and seven patients $(5.3 \%)$ received other treatments.

\section{Measures}

\section{SCID-I}

As part of the routine diagnostic procedure at the clinical site, Axis-I diagnosis was assessed at an initial diagnostic assessment using the SCID-I (First et al., 1996). The interview was administered by trained master or doctoral-level psychologists, psychotherapists, psychiatrists, and senior psychiatric residents.

\section{$B D I-I I$}

Depression severity was assessed using the BDI-II (Beck et al., 1996), a 21-item selfreport instrument assessing depressive symptoms during the last two weeks. Each item is represented by four statements in terms of increasing severity. For each statement a score of $0-4$ is assigned resulting in a total score of 0 to 63 . In the present study the Dutch version of the BDI-II was used, which was shown to possess high internal consistency in a Dutch sample of psychiatric patients (Cronbach's $\alpha=.92$ ) as well as adequate construct validity with related depression rating scales ( $r$ s between .79 - .85; van der Does, 2002). In the present study, internal reliability coefficient alpha for the BDI-II total score was excellent both at time $1(\alpha=.87)$ and at time $2(\alpha=.94)$.

\section{Schema Questionnaire}

The Schema Questionnaire (SQ) is a 205-item self-report instrument, designed to assess 16 specific EMSs (Young and Brown, 1994). 5 These 16 EMSs are (1) abandonment/instability, (2) defectiveness/shame, (3) emotional deprivation, (4) mistrust/abuse, (5) social isolation, (6) dependence/incompetence, (7) vulnerability to harm and illness, (8) enmeshment, (9) failure to achieve, (10) social undesirability, (11) entitlement/grandiosity, (12) insufficient self-control/selfdiscipline, (13) self-sacrifice, (14) subjugation, (15) emotional inhibition, and (16) unrelenting standards. See Table 1 for a brief description of each EMS. The EMS social undesirability is no longer

\footnotetext{
${ }^{4}$ Bonferonni-corrected significance level $\alpha: .05 / 15$

${ }^{5}$ Although to date 18 EMSs were identified, the 205-item version of the SQ that was used in the current study does only measure 16 EMSs. This is because the listing of EMSs has been updated more recently than the SQ that was used in this study. The EMSs that are not covered by the 205-item SQ are approvalseeking/recognition-seeking, negativity/pessimism, and punitiveness.
} 
considered a separate schema in schema theory and is therefore omitted from the present study. Thus, the present report is based on 15 EMSs covered by the 205 -items version of the SQ. Each item is phrased as a negative core belief regarding oneself or ones relation to others. Items are rated along a 6 point scale ranging from 1 (Completely untrue of me) to 6 (Describes me perfectly). In the present study the Dutch version of the original 205-item version was used (Sterk and Rijkeboer, 1997). High internal reliability was reported in a Dutch sample of psychiatric patients for all SQ subscales (Cronbach's $\alpha$ $=.74$ - .92; Rijkeboer and van den Bergh, 2006). In the present study internal reliability coefficient alpha of the SQ was good to excellent for all subscales both at time 1 (median $\alpha=.88$; range $.78-.92$ ) and at time 2 (median $\alpha=.92$; range .78 - .94).

\section{Procedure}

Patients who were referred to the treatment program underwent an intake procedure consisting of an open interview as part of the general intake procedure at the clinic and a SCID I interview (First et al., 1996). Moreover, patients received verbal and written information about evidence-based treatment options at the clinic. To formulate treatment recommendations, clinical history and diagnoses were discussed in an interdisciplinary team meeting. The final choice of treatment modality was taken in agreement between patient and therapist. In some cases (e.g., chronic depression) the therapist actively advised ADM in combination with psychotherapy. ADM treatment consisted of selective serotonin reuptake inhibitors (SSRIs) according to national and international guidelines (American Psychiatric Association, 2000; National Institute of Clincial Evidence, 2004) or in case of previous SSRI non-response venlafaxine or a tricyclic agent. CT and IPT consisted of weekly therapy sessions (50 minutes each) by experienced therapists, with the possibility of biweekly booster sessions in later stages. CT was provided according to the treatment manual by Beck et al., (1979) and IPT was provided according to the treatment manual by Klerman et al., (1984). All therapists received appropriate training and had weekly meetings to discuss ongoing cases and difficulties. Patients who were willing to participate in the study provided written informed consent. Time 1 measures were obtained before the patient started with treatment and time 2 measures were obtained 16-weeks after the initial assessment.

\section{Statistical Analyses}

To test hypothesis 1, we first computed Pearson correlations between the total scores on the five schema domains and the BDI-II at time 1 . We then conducted a stepwise multivariate regression analysis with a backward deletion procedure. In all multivariate regression analyses with backward deletion procedures, a predictor was removed from the model when the partial $F$ value reached a significance level of $\alpha>.10$ and the model was rerun with the respective predictor omitted. In the first analysis, the five schema domains at time 1 were simultaneously entered as predictors and the BDI-II total score at time 1 was entered as dependent variable. We repeated this analysis entering four 
schema domains in the regression analysis because it has been suggested that four schema domains provide a better description of the underlying EMS (Hoffart et al., 2005). Following this analysis, a second multivariate regression analysis was conducted with specific EMSs at time 1 as predictors and BDI-II total scores at time 1 as dependent variable. Given the limited sample size in the current study and the relative large amount of predictors (15 EMSs) we decided to only enter those EMSs belonging to the schema domains that were significantly related to depressive symptom severity in the first regression analysis.

To test hypothesis 2, we conducted a hierarchical multivariate linear regression analysis with the BDI-II total score at time 2 as dependent variable. In the first step, the BDI-II total score at time 1 was entered in the model. In the second step, the mean scores on the 5 schema domains at time 1 were entered. Given the limited sample size for the time 2 assessments, we did not conduct a separate analysis for specific time 1 EMSs as predictors of the time 2 BDI-II total score.

To test hypothesis 3, we followed the analytic procedure emphasized by previous research on the stability of EMSs (e.g., Riso et al., 2006; Wang et al., 2010). First, Pearson correlations between EMSs at time 1 and EMSs at time 2 were calculated. Then, rank order stability was derived from standardized beta weights of multivariate regression models after controlling for depression severity at both time points. We used separate regression equations for each individual EMS with the time 2 EMS score as dependent variable, the time 1 EMS score as predictor and the BDI-II total score at both time points as covariates. High relative stability in these analyses is indicated by large test-retest correlations and large standardized beta coefficients (Santor et al., 1997). To determine the absolute stability of EMSs we conducted a series of paired-sample $t$-tests with a Bonferroni adjusted significance level alpha (.05 / 15) to control for multiple testing. We also computed effect sizes for all mean differences using Cohen's $d$. In these analyses, statistically significant mean differences in EMSs between the two time points are indicative for poor absolute stability. We also determined effects of the different treatment conditions on change in depressive symptom severity and change in EMSs by including dummy coded variables for the different treatment conditions in the regression models of change described above. In these models the CT condition was used as the reference condition.

\section{Results}

First, we examined diagnostic statistics to test for assumptions of linear regression analyses. Although all schema domains displayed statistically significant and moderate to strong intercorrelations ( $r$ s between $.51-.75$ ), the assumption of non-perfect collinearity was met (Tolerance was $>.2$ and VIF < 10) for all regression models; Visual inspection of normality plots suggested that error terms were normally distributed; The assumption of independent errors was met (Durbin Watson test between 1.81 and 2.01); All standardized residuals were in the range of -3 and 3 , suggesting that there were no outliers or influential cases. 
Concurrent relations between depressive symptom severity, schema domains and specific EMSs - Hypothesis 1

Table 2 shows means, standard deviations, and Pearson correlations between BDI-II total scores at time 1 and the five schema domains at time 1 . All schema domains correlated highly and statistically significantly with the BDI-II total score at time 1 . The mean endorsement of the five schema domains at time 1 ranged from 2.49 to 3.14 . To date there are no norm scores available for the SQ. The mean endorsement of schema domains in the present study is comparable to the mean endorsement of schema domains previously reported in depressed samples (e.g., Halvorsen et al., 2009).

Table 2. Means, standarddeviations, and Pearson correlations between BDI-II scores and the five schema domains at time 1 .

\begin{tabular}{|c|c|c|c|c|c|c|c|}
\hline Measure & 1 & 2 & 3 & 4 & 5 & 6 & 7 \\
\hline 1. T1 BDI-II & $\begin{array}{l}29.42 \\
(10.33)\end{array}$ & & & & & & \\
\hline 2. T1 Total schemas & .63 & $\begin{array}{l}2.71 \\
(0.73)\end{array}$ & & & & & \\
\hline 3. T1 Disconnection \& Rejection & .60 & .94 & $\begin{array}{l}2.72 \\
(0.93)\end{array}$ & & & & \\
\hline 4. T1 Impaired Autonomy \& performance & .59 & .89 & .73 & $\begin{array}{l}2.49 \\
(0.75)\end{array}$ & & & \\
\hline 5. T1 Impaired Limits & .41 & .80 & .71 & .68 & $\begin{array}{l}2.59 \\
(0.73)\end{array}$ & & \\
\hline 6. T1 Other-directedness & .54 & .83 & .71 & .73 & .51 & $\begin{array}{l}3.14 \\
(0.84)\end{array}$ & \\
\hline 7. T1 Overvigilance \& Inhibition & .50 & .86 & .75 & .70 & .70 & .71 & $\begin{array}{l}2.81 \\
(0.84)\end{array}$ \\
\hline
\end{tabular}

Note. Off-diagonal shows correlation coefficients; Diagonal shows means and standarddeviations; $N=132$; $\mathrm{T} 1$ = time 1; BDI-II = Beck Depression Inventory-II. All correlation coefficients are significant $(p<.001$; two-tailed).

Table 3 summarizes the results of the stepwise multivariate regression analysis. The BDI-II total score at time 1 was entered as dependent variable and the five schema domains at time 1 as independent variables. In the final model, the schema domains impaired autonomy \& performance and disconnection \& rejection remained as significant predictors of BDI-II total scores at time $1, \beta=.34, t(129)=3.48, p<.001$, and $\beta=.35, t(129)=3.53, p<.001$, respectively. Together these two schema domains explained $41 \%$ of the variance in BDI-II total scores at time 1 . We repeated the analysis with the four schema domains proposed by Hoffart et al. (2005) and again found that impaired autonomy \& performance and disconnection \& rejection remained as 
significant predictors of BDI-II total scores at time $1, \beta=.40, t(129)=3.86, p<.001$, and $\beta=.43, t(129)=3.92, p<.001$, respectively.

Table 3. Summary of multivariate linear regression analysis with backward deletion predicting time 1 depressive symptom severity with schema domains at time 1 .

\begin{tabular}{|c|c|c|c|c|c|c|c|c|}
\hline & Model 1 & & Model 2 & & Model 3 & & Model 4 & \\
\hline Predictor & $B(S E)$ & $\beta$ & $B(S E)$ & $\beta$ & $B(S E)$ & $\beta$ & $B(S E)$ & $\beta$ \\
\hline Constant & $7.72(3.09)^{*}$ & & 7.75 (3.07)* & & $8.97(2.71)^{* *}$ & & $7.12(2.49)^{* *}$ & \\
\hline $\begin{array}{l}\text { T1 Overvigilance \& } \\
\text { Inhibition }\end{array}$ & $0.38(1.46)$ & .03 & & & & & & \\
\hline $\begin{array}{l}\text { T1 Other } \\
\text { Directedness }\end{array}$ & $1.01(1.42)$ & .08 & $1.13(1.34)$ & .09 & & & & \\
\hline T1 Impaired Limits & $-2.34(1.56)$ & -.16 & $-2.21(1.48)$ & -.16 & $-2.42(1.45)$ & -.17 & & \\
\hline $\begin{array}{l}\text { T1 Impaired } \\
\text { Autonomy \& } \\
\text { Performance }\end{array}$ & $4.88(1.65)^{* *}$ & .35 & $4.91(1.64)^{* *}$ & .36 & $5.56(1.44)^{* *}$ & .40 & $4.74(1.36)^{* *}$ & .34 \\
\hline $\begin{array}{l}\text { T1 Disconnection \& } \\
\text { Rejection }\end{array}$ & $4.17(1.38)^{* *}$ & .38 & $4.26(1.33)^{* *}$ & .38 & $4.72(1.21)^{* *}$ & .43 & $3.85(1.09)^{* *}$ & .35 \\
\hline$R^{2}$ & .43 & & .43 & & .42 & & .41 & \\
\hline$\Delta R^{2}$ & .43 & & .00 & & -.01 & & -.01 & \\
\hline
\end{tabular}

Note. $N=132 ; \mathrm{T} 1=$ Time $1 ;{ }^{*} p<.05 ;{ }^{* *} p<.01$

To determine which specific EMSs from the impaired autonomy \& performance and disconnection \& rejection domains were related to depressive symptom severity, all EMSs from these two domains were included in another multivariate stepwise regression analysis. Table 4 summarizes the results of the initial model with all EMSs as predictors, the next to final model, and the final model. In the final model, three specific EMSs remained as significant predictors of depressive symptom severity: abandonment/instability, $\beta=.31, t(127)=2.99, p=.003$, failure, $\beta=.41, t(127)=5.11, p$ $<.001$, and emotional deprivation, $\beta=.19, t(127)=2.22, p=.028$. Moreover, the EMS enmeshment remained as a marginally significant predictor of depressive symptom severity, $\beta=-.14, t(127)=-1.83, p=.07$. The final model explained $48 \%$ of the variance in BDI-II total scores at time 1.

Relation between EMSs at time 1 and depressive symptom severity at time 2 - Hypothesis 2

Table 5 summarizes the results of the multivariate hierarchical regression model predicting reduction in depressive symptoms with the five schema domains at time 1. The BDI-II total score at time 2 was entered as dependent variable. The BDI-II total score at time 1 was entered as predictor at step 1 and the five schema domains at time 1 were simultaneously entered as predictors at step 2 . At step 1 , time 1 depression severity was 
a significant predictor of time 2 depression severity, $\beta=.75, t(83)=10.29, p<.001$, explaining $56 \%$ of the variance in the BDI-II total score at time 2 . Adding the five schema domains at step 2 significantly improved the hierarchical regression model, $\Delta R^{2}=.06, p$ $=.035$. At step 2 , time 1 scores on the overvigilance $\&$ inhibition domain were negatively related to the BDI-II total score at time $2, \beta=-.29, t(78)=-2.20, p=.031$. Moreover, there was a marginally significant positive relation between the impaired autonomy \& performance domain and time 2 depression severity, $\beta=.27, t(78)=1.97, p=.053$.

Table 4. Summary of multivariate linear regression analysis with backward deletion predicting time 1 depressive symptom severity with domain specific EMSs at time 1.

\begin{tabular}{|c|c|c|c|c|c|c|}
\hline \multirow[b]{2}{*}{ Predictor } & \multicolumn{2}{|l|}{ Model 1} & \multicolumn{2}{|l|}{ Model 5} & \multicolumn{2}{|l|}{ Model 6} \\
\hline & $B(S E)$ & $\beta$ & $B(S E)$ & $\beta$ & $B(S E)$ & $\beta$ \\
\hline Constant & $7.91(2.49)^{* *}$ & & $7.92(2.47)^{* *}$ & & $8.06(2.32)$ & \\
\hline T1 Dependence/Incompetence ${ }^{a}$ & $0.08(1.28)$ & .01 & & & & \\
\hline T1 Vulnerability to Harma & $0.41(1.18)$ & .03 & & & & \\
\hline T1 Defectiveness/Shame ${ }^{b}$ & $1.19(1.48)$ & .11 & & & & \\
\hline T1 Social Inhibition ${ }^{\mathrm{b}}$ & $-1.40(1.11)$ & -.16 & & & & \\
\hline T1 Mistrust/Abuse ${ }^{b}$ & $0.95(1.14)$ & .09 & $1.10(1.08)$ & .11 & & \\
\hline T1 Enmeshment ${ }^{\mathrm{a}}$ & $-1.78(0.95)$ & -.15 & $-1.66(0.92)$ & -.14 & $-1.68(0.92)$ & -.14 \\
\hline T1 Emotional Deprivation ${ }^{\mathrm{b}}$ & $1.50(0.93)$ & .17 & $1.22(0.84)$ & .14 & $1.63(0.73)^{*}$ & .19 \\
\hline T1 Abandonment/Instabilitya & $2.55(1.29)$ & .26 & $2.60(1.14)^{*}$ & .26 & $3.09(1.03)^{* *}$ & .31 \\
\hline T1 Failure ${ }^{\mathrm{a}}$ & $3.95(.98)^{* *}$ & .41 & $3.98(0.77)^{* *}$ & .42 & $3.96(0.77)^{* *}$ & .41 \\
\hline$R^{2}$ & .49 & & .48 & & .48 & \\
\hline$\Delta R^{2}$ & .49 & & -.01 & & .00 & \\
\hline
\end{tabular}

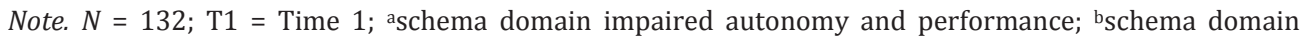
disconnection and rejection; ${ }^{*} p<.05 ;{ }^{* *} p<.01$.

Table 5. Summary of multivariate hierarchical regression analyses testing whether schema domains at time 1 predict depressive symptom severity at time 2 .

\begin{tabular}{|c|c|c|c|c|c|c|}
\hline Step & Predictor & $B(S E)$ & $\beta$ & $t$ & $R^{2}$ & $\Delta R^{2}$ \\
\hline Step 1 & T1 BDI-II & $0.95(0.09)$ & .75 & $10.29^{* *}$ & .56 & .56 \\
\hline \multirow[t]{6}{*}{ Step 2} & T1 BDI-II & $0.77(0.12)$ & .61 & $6.34^{* *}$ & .62 & .06 \\
\hline & T1 Impaired Limits & $-1.04(2.11)$ & -.06 & -.49 & & \\
\hline & T1 Other Directedness & $.01(2.02)$ & .00 & .01 & & \\
\hline & T1 Disconnection \& Rejection & $3.61(1.93)$ & .25 & 1.87 & & \\
\hline & T1 Impaired Autonomy \& Performance & $4.72(2.40)$ & .27 & 1.97 & & \\
\hline & T1 Overvigilance \& Inhibition & $-4.74(2.15)^{*}$ & -.29 & -2.20 & & \\
\hline
\end{tabular}

Note. $N=85 ; \mathrm{T} 1$ = Time 1; BDI-II = Beck Depression Inventory-II; ${ }^{*} p<.05 ;{ }^{* *} p<.01$. 
Stability of EMSs over the course of treatment - Hypothesis 3

\section{Absolute stability}

The absolute stability of EMSs over the course of treatment was determined by paired sample $t$-tests. The results of this analysis are summarized in Table 6. After controlling for multiple testing, there was a statistically significant but small decrease in the total SQ score_from time 1 to time $2, t(84)=4.50, p=.003, d=0.29$. Moreover, patients statistically significantly improved on 10 EMSs (Abandonment/instability, mistrust/abuse, emotional deprivation, dependence/incompetence, vulnerability to harm, insufficient self-control, subjugation, self-sacrifice, emotional inhibition, unrelenting standards) with small to medium effect sizes (Cohen's $d$ between $0.15-0.35$; Cohen, 1988). To compare stability of EMSs to symptom level stability, we also computed change in BDI-II total scores and found a statistically significant decrease in BDI-II total scores from time $1(M=29.55, S D=10.63)$ to time $2(M=21.24 ; S D=13.42)$, $t(84)=8.59, p<.001, d=0.69$.

\section{Relative stability}

The relative stability of EMSs over the course of treatment was determined by correlational and regression analyses. Table 6 shows stability correlations between EMSs at both time points. Time 1 EMSs subscales correlated highly and statistically significantly with time 2 EMSs subscales (Bivariate stability coefficients $r$ between 68 and .87, $p<.001$ ). After controlling for depression severity at both time points in regression analyses, the stability coefficient $(\beta)$ decreased slightly but remained statistically significant ( $\beta$ s between .58 and $.82, p<.001$ ) suggesting that EMSs exhibit high relative stability over the course of 16 -weeks outpatient treatment for depression.

\section{Differences in change of depressive symptoms and change in EMSs between treatment conditions}

Adding the different treatment conditions to the regression model of change in depressive symptom severity did not significantly improve the model $\left(\Delta R^{2}=.04, p=.59\right)$. Moreover, there was no differences in change of the total SQ score between treatment conditions (all $p$-values $>.05$ ). With respect to specific EMSs, there was less change in the EMS self-sacrifice in the IPT condition compared to the CT condition, $\beta=.14, t(84)=$ $1.96, p=.05$ and there was less change in the EMS unrelenting standards in the othertreatments condition compared to the CT condition, $\beta=.13, t(84)=2.03, p<.05$.

\section{Differences in change in EMSs between treatment responders and treatment non- responders}

We also determined the relative stability of EMSs between treatment responders (defined as a drop in BDI-II levels by more than 50\%) and treatment non-responders. Non-responders reported greater change on the EMS social inhibition compared to 
responders, $\beta=-.17, t(84)=-2.27, p<.05$. Change scores on the other EMSs did not differ statistically significantly between responders and non-responders (all $p$ values $>$.05).

Table 6. Relative and absolute stability of EMSs from time 1 to time 2.

\begin{tabular}{|c|c|c|c|c|c|c|c|}
\hline \multirow[b]{3}{*}{ Variable } & \multicolumn{2}{|c|}{ Relative stability } & \multicolumn{2}{|c|}{ Absolute stability } & \multirow[b]{3}{*}{$\begin{array}{l}\text { Paired } \\
t \text { test }\end{array}$} & \multirow[b]{3}{*}{$d f$} & \multirow[b]{3}{*}{$d$} \\
\hline & & & Time 1 & Time 2 & & & \\
\hline & Stability $^{6}$ & Stability $^{7}$ & $M(S D)$ & $M(S D)$ & & & \\
\hline SQ total & $.82^{* *}$ & $.62^{* *}$ & $2.62(0.76)$ & $2.39(0.80)$ & $4.50^{* *}$ & 84 & 0.29 \\
\hline Abandonment/Instability & $.80^{* *}$ & $.63^{* *}$ & $2.76(1.03)$ & $2.46(1.06)$ & $4.12^{* *}$ & 84 & 0.29 \\
\hline Mistrust/Abuse & $.85^{* *}$ & $.75^{* *}$ & $2.57(1.02)$ & $2.36(1.05)$ & $3.44^{*}$ & 84 & 0.20 \\
\hline Emotional Deprivation & $.87^{* *}$ & $.82^{* *}$ & $2.75(1.21)$ & $2.49(1.24)$ & $3.88^{* *}$ & 84 & 0.21 \\
\hline Defectiveness/Shame & $.78^{* *}$ & $.58^{* *}$ & $2.22(0.96)$ & $2.07(0.99)$ & 2.05 & 84 & 0.15 \\
\hline Social Isolation/Alienation & $.79^{* *}$ & $.66^{* *}$ & $2.85(1.20)$ & $2.60(1.23)$ & 2.85 & 84 & 0.21 \\
\hline Dependence/Incompetence & $.79^{* *}$ & $.59^{* *}$ & $2.51(0.98)$ & $2.29(0.96)$ & $3.15^{*}$ & 84 & 0.23 \\
\hline Vulnerability to Harm & $.81^{* *}$ & $.70^{* *}$ & $2.26(0.86)$ & $1.97(0.79)$ & $5.18^{* *}$ & 84 & 0.35 \\
\hline Enmeshment & $.74^{* *}$ & $.68^{* *}$ & $2.05(0.89)$ & $1.90(0.96)$ & 2.07 & 84 & 0.16 \\
\hline Failure & $.77^{* *}$ & $.63^{* *}$ & $2.78(1.09)$ & $2.59(1.22)$ & 2.22 & 84 & 0.16 \\
\hline Entitlement/Grandiosity & $.68^{* *}$ & $.62^{* *}$ & $2.18(0.69)$ & $2.01(0.68)$ & 2.96 & 84 & 0.25 \\
\hline Insufficient Self-Control & $.80^{* *}$ & $.70^{* *}$ & $2.74(0.89)$ & $2.47(0.94)$ & $4.22^{* *}$ & 84 & 0.29 \\
\hline Subjugation & $.80^{* *}$ & $.71^{* *}$ & $2.77(1.04)$ & $2.57(1.02)$ & $2.70^{*}$ & 84 & 0.19 \\
\hline Self-Sacrifice & $.79^{* *}$ & $.65^{* *}$ & $3.40(0.90)$ & $3.18(1.00)$ & $3.35^{*}$ & 84 & 0.23 \\
\hline Emotional Inhibition & $.81^{* *}$ & $.72^{* *}$ & $2.62(1.05)$ & $2.29(1.02)$ & $4.70^{* *}$ & 84 & 0.32 \\
\hline Unrelenting Standards & $.79^{* *}$ & $.77^{* *}$ & $2.80(0.90)$ & $2.52(0.94)$ & $4.22^{* *}$ & 84 & 0.30 \\
\hline
\end{tabular}

Note. $N=85 ; \mathrm{SQ}=$ Schema questionnaire; Effect sizes $(d s)$ were calculated by dividing the mean difference with the pooled standard deviation and may be interpreted as small to medium (Cohen, 1988). ${ }^{*} p<.05$ (Corrected for multiple testing); ${ }^{* *} p<.01$ (Corrected for multiple testing).

\section{Discussion}

The aims of this study were to relate EMSs to depressive symptom severity during a course of outpatient treatment for depression and to determine the relative and absolute stability of EMSs over the course of treatment. We found that, after controlling for overlap among schema domains, EMSs from the domains impaired autonomy \& performance and disconnection \& rejection were related to depressive symptom

\footnotetext{
${ }^{6}$ Bivariate stability correlations.

${ }^{7}$ Stability coefficient after controlling for depression severity at both time points in regression analyses.
} 
severity in a naturalistic sample of outpatients suffering from MDD. This finding is largely in line with previous findings relating EMSs to depressive symptom severity (e.g., Calvete et al., 2005; Harris and Curtin, 2002) and suggests that depressed patients are characterized by a specific set of EMSs. In particular, in cross-sectional analyses we found that the EMSs abandonment/instability, emotional deprivation, and failure were positively related to depressive symptom severity when controlling for overlap among EMSs. Moreover, the EMS enmeshment was marginally significantly negatively related to depressive symptom severity. These four EMSs accounted for $48 \%$ of the variance in depressive symptom severity with the failure schema accounting for the largest part. It should be noted, however, that there were weak to strong intercorrelations ( $r$ s between .27 - .64) among these four EMSs which makes it difficult to determine the individual importance of each predictors. These findings are consistent with the cognitive model of depression, placing schemas or core beliefs in the domains of failure, loss, and worthlessness at the core of depressive symptoms (Beck, 1964, 1987).

We also investigated the validity of schema domains at pre-treatment as predictors of improvement in depressive symptom severity over the course of treatment and found that the schema domain overvigilance \& inhibition was negatively related to depressive symptom severity whereas the schema domain impaired autonomy \& performance was positively related to depressive symptom severity assessed 16-weeks after the start of treatment. The relation between impaired autonomy \& performance and depressive symptom severity at time 2 was slightly below the threshold of statistical significance and should therefore be interpreted with caution. The finding that EMSs from the overvigilance \& inhibition domains at pre-treatment were negatively related to depressive symptom severity at the time 2 assessment was unexpected and is difficult to explain in the context of schema theory. Patients with high scores on this EMS domain are typically characterized by a suppression of spontaneous feelings and impulses or by a preoccupation of meeting high internalized standards of performance (Young et al., 2003). One possible, explanation of this finding is that patients who score high on this schema domain might tend to deny or inhibit their negative emotions in self-report of depressive symptoms. Hence, they might report less depressive symptoms at time 2 because of a high inhibition of emotions. An alternative explanation, suggested by one of the anonymous reviewers, is that patients with high scores on this schema domain work harder in therapy because of their high internalized standards of performance and therefore obtain better results. Both explanations are speculative and require further testing. The finding that impaired autonomy \& performance levels at pre-treatment were positively related to depressive symptoms at time 2 suggest that depressed patients with increased expectations about themselves and others that interfere with their perceived ability to function independently or perform successfully show less symptom improvement in treatment for depression. However, given the relatively low percentage of explained variance in BDI-II scores, the robustness of these findings is questionable and replication in more controlled studies is needed. Moreover, it should be noted that studies on the psychometric properties of the SQ have yielded mixed results regarding the higher-order factor structure (Hoffart et al., 2005; Lee et al., 1999; 
Schmidt et al., 1995; Soygüt et al., 2009). Therefore, our findings regarding the predictive validity of schema domains should be interpreted with caution.

Consistent with our third hypothesis, we found high relative stability of EMSs from time 1 to time 2 in the context of statistically significant and large decreases in depressive symptom severity. Moreover, the relative stability of EMSs remained high when controlling for depressive symptom severity at both time points. Given that none of the treatment protocols in the present study explicitly target EMSs this finding was expected. In terms of absolute stability, we found a statistically significant but small (Cohen's $d$ between 0.15 - 0.35) decrease in 10 EMSs (Abandonment/instability, mistrust/abuse, emotional deprivation, dependence/incompetence, vulnerability to harm, insufficient self-control, subjugation, self-sacrifice, emotional inhibition, unrelenting standards) whereas decrease in depressive symptom severity was statistically significant and moderate (Cohen's $d=0.69$ ). Given the high relative stability and the low effect sizes for the mean differences, our findings suggest that EMSs remain stable in depressed patients over a course of outpatient treatment. This finding is consistent with earlier research on the stability of EMSs in depressed patients (Riso et al., 2006; Wang et al., 2010) and the notion that EMSs represent stable, trait-like constructs (Young et al., 2003). Moreover, our results extend previous findings on the stability of EMSs in depression that have primarily focused on the long-term stability of EMSs (Riso et al., 2006; Wang et al., 2010) to the relative stability of EMSs in the context of symptom change.

We also determined the stability of EMSs in the different treatment conditions and found no substantial differences between treatment conditions, except for the EMS selfsacrifice which changed less in the IPT condition compared to the CT condition and for the EMS unrelenting standards which changed less in the other-treatments condition, compared to the CT condition. However, given the lack of random assignment to treatment conditions in the current study, these results should be interpreted with caution. Overall, our results indicate that there are no substantial differences in change in EMSs across treatment conditions. There is a need to replicate these findings in more controlled settings.

\section{Limitations}

First, we did not include a clinical control group with psychopathology other than depression in this study and therefore no conclusions regarding the specificity of our findings can be drawn. Second, we did not assess Axis-II psychopathology. Given that patients with Axis-II psychopathology generally exhibit high scores of EMSs (Nordahl et al., 2005), it is desirable to assess and control for Axis-II comorbidity when studying the relation between EMSs and depressive symptoms. Third, we determined cross-sectional relations between EMSs and depressive symptoms. While theory would suggest that EMSs drive depression, the nature of our research design and analyses does not allow for drawing causal conclusions from our findings. Fourths, in this study no other measures of dysfunctional cognitions was obtained. It would be interesting to 
investigate how EMSs relate to depressive symptoms over a course of treatment, relative to measures of dysfunctional surface cognitions, like automatic thoughts. Fifth, the sample in the current study was characterized by relatively severe levels of depression and hence the range of BDI-II scores was restricted, which might have led to an underestimation of correlation coefficients between BDI-II and EMSs scores. Finally, we did not assess the recently identified EMS approval-seeking/recognition-seeking, negativity/pessimism, and punitiveness that are covered by the latest version of the SQ (Young, 2006). Future research on the relation between EMS and depressive symptoms should also assess these additional EMS.

\section{Clinical Implications}

Despite these limitations the present study has important implications for clinical settings and future research. First, we found that specific EMSs were related to depressive symptom severity in depressed patients. This finding needs further replication in more controlled settings with clinical control groups in order to determine the specificity of these EMSs to depression. We also found that schema domains at pretreatment were related to change in depressive symptom severity over the course of treatment. Therefore, it might be valuable, for prognostic reasons, to assess EMSs prior to treatment. Future studies should also address the causal direction of the relation between EMSs and change in depressive symptoms. For example, it would be interesting to test whether change in EMSs is a possible mechanism that leads to improvement from depressive symptoms, as theory would suggest.

Our results suggest that EMSs in depressed patients remain relatively stable in the context of change in depressive symptoms during evidence-based short-term treatments for depression. One consequence might be that patients leave treatment with reduced symptomatology but with a largely unaltered underlying vulnerability for future depressive episodes. This might be especially problematic for depressed patients with high levels of EMSs and might lead to an increased risk for subsequent relapse. For example, it has been shown that chronically depressed patient exhibit statistically significantly higher scores on all schema domains as compared to non-chronically depressed patients (Riso et al., 2003). Given that EMSs are likely to represent a core vulnerability factor to psychopathology, depressed patients with highly dysfunctional EMSs (i.e., chronically depressed patients) might benefit from long-term treatments that are specifically designed to alter underlying EMSs, such as schema-focused therapy (Young et al., 2003).

In conclusion, the current study provides further empirical support that specific EMSs are related to depressive symptoms and that these dysfunctional schemas are robust to change, even in the context of evidence-based outpatient treatment for depression. Moreover, our findings suggest that specific schema domains assessed before treatment predict depressive symptom severity 16-weeks after the initial assessment. Replication of these findings in more controlled settings is needed. 


\section{References}

American Psychiatric Association, 1994. Diagnostic and Statistical manual of mental disorders (4th ed.). Washington, D.C.

American Psychiatric Association, 2000. Practice guideline for the treatment of patients with major depressive disorder (revision). Am. J. Psychiatry 157, 1-45.

Beck, A.T., 1964. Thinking and depression: II. Theory and therapy. Arch. Gen. Psychiatry 10, 561-571.

Beck, A.T., 1987. Cognitive models of depression. Journal of Cognitive Psychotherapy 1, 5-37.

Beck, A.T., Rush, A.J., Shaw, B.F., Emery, G., 1979. Cognitive therapy of depression. Guilford, New York.

Beck, A.T., Steer, R.A., Brown, G.K., 1996. Manual for the Beck Depression Inventory-II, San Antonio, TX.

Beevers, C.G., Miller, I.W., 2004. Depression-Related Negative Cognition: Mood-State and Trait Dependent Properties. Cognitive Therapy \& Research 28, 293-307.

Calvete, E., Estévez, A., López de Arroyabe, E., Ruiz, P., 2005. The Schema Questionnaire--Short Form: Structure and Relationship with Automatic Thoughts and Symptoms of Affective Disorders. European Journal of Psychological Assessment 21, 90-99.

Clark, D.M., Beck, A.T., 1999. Scientific foundations of cognitive theory and therapy for depression. Wiley., New York.

Cohen, J., 1988. Statistical power analysis for the behavioral sciences. Erlbaum, Hillsdale, NJ.

First, M.B., Spitzer, R.L., Gibbon, M., Williams, J.B., 1996. Structured Clinical Interview for DSM-IV Axis I Disorders-Patient Edition (SCID-I/P, Version 2.0). New York State Psychiatric Institute, Biometrics Research Department, New York.

Halvorsen, M., Wang, C., Eisemann, M., Waterloo, K., 2009. Dysfunctional Attitudes and Early Maladaptive Schemas as Predictors of Depression: A 9-Year Follow-Up Study. Cognitive Therapy and Research 16, 394-407.

Harris, A.E., Curtin, L., 2002. Parental perceptions, early maladaptive schemas, and depressive symptoms in young adults. Cognitive Therapy and Research 26, 405-416.

Hoffart, A., Sexton, H., Hedley, L.M., Wang, C.E., Holthe, H., Haugum, J.A., Nordahl, H.M., Hovland, O.J., Holte, A., 2005. The Structure of Maladaptive Schemas: A Confirmatory Factor Analysis and a Psychometric Evaluation of Factor-Derived Scales. Cognitive Therapy and Research 29, 627-644.

James, I.A., Southam, L., Blackburn, I.M., 2004. Schemas Revisited. Clinical Psychology \& Psychotherapy 11, 369-377.

Klerman, G., Weissman, M., Rounsaville, B., Chevron, E., 1984. Interpersonal psychotherapy of depression. Basic Books, New York.

Lee, C.W., Taylor, G., Dunn, J., 1999. Factor structure of the Schema Questionnaire in a large clinical sample. Cognitive Therapy and Research 23, 441-451.

National Institute of Clincial Evidence, 2004. Depression: Management of depression in primary and secondary care., London.

Nordahl, H.M., Holthe, H., Haugum, J.A., 2005. Early Maladaptive Schemas in Patients with or without Personality Disorders: Does Schema Modification Predict Symptomatic Relief? Clinical Psychology \& Psychotherapy 12, 142-149.

Petrocelli, J.V., Glaser, B.A., Calhoun, G.B., Campbell, L.F., 2001. Cognitive schemas as mediating variables of the relationship between the self-defeating personality and depression. J. Psychopathol. Behav. Assess. 23, 183-191.

Rijkeboer, M.M., van den Bergh, H., 2006. Multiple group confirmatory factor analysis of the Young Schema-Questionnaire in a Dutch clinical versus non-clinical population. Cognitive Therapy and Research 30, 263-278.

Riso, L.P., du Toit, P.L., Blandino, J.A., Penna, S., Dacey, S., Duin, J.S., Pacoe, E.M., Grant, M.M., Ulmer, C.S., 2003. Cognitive aspects of chronic depression. J. Abnorm. Psychol. 112, 72-80.

Riso, L.P., Froman, S.E., Raouf, M., Gable, P., Maddux, R.E., Turini-Santorelli, N., Penna, S., Blandino, J.A., Jacobs, C.H., Cherry, M., 2006. The Long-Term Stability of Early Maladaptive Schemas. Cognitive Therapy and Research 30, 515-529. 
Santor, D.A., Bagby, R.M., Joffe, R.T., 1997. Evaluating stability and change in personality and depression. J. Pers. Soc. Psychol. 73, 1354-1362.

Schmidt, N.B., Joiner, T.E., Young, J.E., Telch, M.J., 1995. The Schema Questionnaire: Investigation of psychometric properties and the hierarchical structure of a measure of maladaptive schemas. Cognitive Therapy and Research 19, 295-321.

Segal, Z.V., 1988. Appraisal of the self-schema construct in cognitive models of depression. Psychol. Bull. $103,147-162$.

Segal, Z.V., Swallow, S.R., 1994. Cognitive assessment of unipolar depression: Measuring products, processes and structures. Behav. Res. Ther. 32, 147-158.

Shah, R., Waller, G., 2000. Parental style and vulnerability to depression: The role of core beliefs. J. Nerv. Ment. Dis. 188, 19-25.

Soygüt, G., Karaosmanoğlu, A., Cakir, Z., 2009. Assessment of Early Maladapive Schemas: A Psychometric Study of the Turkish Young Schema Quesionnaire-Short Form-3. Turk Psikiyatri Dergisi XX, 1-10.

Sterk, F., Rijkeboer, M.M., 1997. Schema-Vragenlijst [Schema-Questionnaire]. Ambulatorium Utrecht University., Utrecht.

Thimm, J.C., 2010. Personality and early maladaptive schemas: A five-factor model perspective. J. Behav. Ther. Exp. Psychiatry 41, 373-380.

van der Does, A.J.W., 2002. Handleiding: De Nederlandse versie van de Beck Depression Inventory, 2de Editie. [The Dutch version of the Beck Depression Inventory, 2nd ed.]. Swets and Zeitlinger b.v., Lisse.

Wang, C.E.A., Halvorsen, M., Eisemann, M., Waterloo, K., 2010. Stability of dysfunctional attitudes and early maladaptive schemas: A 9-year follow-up study of clinically depressed subjects. J. Behav. Ther. Exp. Psychiatry 41, 389-396.

Young, J.E., 1995. Cognitive Therapy for Personality Disorders: A Schema-Focused Approach. Professional Resource Exchange., Sarasota, FL.

Young, J.E., 2006. Young Schema Questionnaire-3. Cognitive Therapy Center, New York.

Young, J.E., Brown, G.S., 1994. Young Schema Questionnaire. In: Young, J.E. (Ed.), Cognitive therapy for personality disorders: A schema-focused approach. Professional Research Exchange, Sarasota, FL.

Young, J.E., Klosko, J., Weishaar, M.E., 2003. Schema therapy: A practitioner's guide. Guilford Press, New York.

Zuroff, D.C., Blatt, S.J., Sanislow, C.A., Bondi, C.M., Pilkonis, P.A., 1999. Vulnerability to Depression: Reexamining State Dependence and Relative Stability. J. Abnorm. Psychol. 108, 76-89. 



\section{CHAPTER 5 \\ Stability of personality traits in treated and untreated patients with depression}

Published as:

Renner, F., Penninx, B. W. J. H., Peeters, F., Cuijpers, P., \& Huibers, M. J. H. (2013). Twoyear stability and change of neuroticism and extraversion in treated and untreated persons with depression: Findings from the Netherlands Study of Depression and Anxiety (NESDA). Journal of Affective Disorders, 150(2), 201-208. 


\begin{abstract}
Background: The personality dimensions neuroticism and extraversion likely represent part of the vulnerability to depression. The stability over longer time periods of these personality dimensions in depressed patients treated with psychological treatment or medication and in untreated persons with depression in the general population remains unclear. Stability of neuroticism and extraversion in treated and untreated depressed persons would suggest that part of the vulnerability to depression remains stable over time. The current study addressed the question whether treatment in depressed patients is related to changes in neuroticism and extraversion.
\end{abstract}

Methods: Data are from 709 patients with major depressive disorder participating in a cohort study (Netherlands Study of Depression and Anxiety; NESDA). We determined the two-year stability of extraversion and neuroticism in treated and untreated persons and related change in depression severity to change in personality over time.

Results: Neuroticism decreased from baseline to two-year follow-up $(d=0.73)$ in both treated and untreated persons. Extraversion did not change significantly after controlling for neuroticism and depression severity at baseline and follow-up. Decreased depressive symptoms over time were related to decreased neuroticism $(d=1.91)$ whereas increased depressive symptoms over time were unrelated to neuroticism $(d=$ 0.06). Limitations: Patients were not randomized to treatment conditions and the groups are therefore not directly comparable.

Conclusions: Treated patients with depression in the general population improve just as much on depression severity and neuroticism as untreated persons with depression. This suggests that changes in neuroticism in the context of treatment likely represent mood-state effects rather than direct effects of treatment.

Keywords: neuroticism; extraversion; major depression; treatment seeking. 


\section{Introduction}

The relation between Major Depressive Disorder (MDD) and the personality dimensions of neuroticism and extraversion has been studied in clinical settings and in population based studies (for an overview: Kotov et al., 2010). These studies have shown that MDD is positively associated with neuroticism and negatively with extraversion. Moreover, clinical and population based studies have identified neuroticism as an important vulnerability factor for MDD (Boyce et al., 1991; Fanous et al., 2007; Hettema et al., 2006; Hirschfeld et al., 1989; Kendler et al., 2006; Kendler et al., 2004; Ormel et al., 2004), whereas low extraversion appears to be associated only weakly to MDD (Kendler et al., 2006).

While neuroticism and extraversion likely represent part of the vulnerability to MDD, they are also known to change with the current mood state. For example, Karsten et al. (2012) used data from the Netherlands Study of Depression and Anxiety (NESDA; Penninx et al., 2008) and found that the occurrence of a depressive disorder was associated with an increase in neuroticism and a decrease in extraversion (Karsten et al., 2012). Similarly recovery from a depressive disorder was associated with decreased neuroticism and increased extraversion (Karsten et al., 2012), suggesting that neuroticism and extraversion are mood state dependent. In this study treatment status was not taken into account so it remains unclear whether treatment is related to the observed changes in neuroticism and extraversion.

The stability of neuroticism and extraversion in patients with MDD has also been studied in the context of randomized clinical trials (RCTs) and in naturalistic treatment studies (Berlim et al., 2013; Ekselius and von Knorring, 1999; Hellerstein et al., 2000; Tang et al., 2009). Studying the stability of neuroticism and extraversion in the context of RCTs is important to determine which aspects of treatment are related to change in personality. Tang et al. (2009), for example, showed that neuroticism decreased and extraversion increased in patients with MDD taking selective serotonin reuptake inhibitors (SSRIs) compared to patients taking a pill placebo. Interestingly, change in personality did not depend on improvement from depressive symptoms suggesting that change in personality is not a mere epiphenomenon of improved depressive state (Tang et al., 2009). In a naturalistic treatment study it has been shown that a 4-week trial of high frequency repetitive transcranial magnetic stimulation was related to decreased levels of neuroticism at post-treatment and the authors suggested that these improvements in neuroticism were likely independent from improvements in depressive state (Berlim et al., 2013). These findings suggest that treatment for MDD may have an impact on the personality of patients with MDD independent of the impact of treatment on depression itself.

In RCTs comparing the impact of depression treatment on change in personality the control condition usually consists of a placebo group (e.g., Ekselius and von Knorring, 1999; Hellerstein et al., 2000; Tang et al., 2009) but patients assigned to placebo usually also experience substantial symptom reduction. To more directly test whether treatment for depression is related to change in personality it is necessary to compare 
treated and untreated persons with depression. This can be done in population based studies because not all depressed persons in the general population seek treatment.

Another issue that has not been addressed in RCTs is the long-term stability of personality in treated and untreated persons with depression. Assessing the long term stability of personality traits in depressed patients is important because stability of personality traits in the context of change in depressive state would suggest that part of the vulnerability to depression remains intact, increasing the risk for subsequent relapse. For example, in a recent meta-analysis of longitudinal studies it has been shown that personality and personality disorders remain relatively stable over time and that patients under treatment for various mental disorders do not report more change in personality than non-patients (Ferguson, 2010). Such findings suggest that part of the vulnerability, in terms of the underlying personality, remains stable over longer periods of time in depressed patients in the general population, regardless of treatment status.

When studying the stability of personality in treated and untreated persons with depression in the general population some differences between RCTs and population based studies should be taken into account. In RCTs patients usually receive highly effective treatments under optimal conditions, whereas at the population level many depressed patients do not always receive adequate health care (Kessler et al., 2003; Young et al., 2001). Moreover, patients with MDD in RCTs might differ from patients with MDD in the general population on a number of clinically relevant variables such as comorbidity.

The present study draws data from the Netherlands Study of Depression and Anxiety (NESDA), to determine the two-year stability of neuroticism and extraversion in patients treated with psychological treatment and/or antidepressant medication and in untreated persons with depression. Moreover, we sought to relate in both untreated and treated persons the change in depressive symptoms over time to change in neuroticism and extraversion over time. The following questions were addressed in this study: 1) Is treatment in patients with MDD at the population level related to change in neuroticism and extraversion? 2) Are changes in depressive symptoms over time in untreated and treated persons with depression related to changes in neuroticism and extraversion?

\section{Methods}

\section{Sample}

The present report is based on data from an ongoing longitudinal cohort study, the Netherlands Study of Depression and Anxiety (NESDA; $N=2981$ ). This ongoing multisite cohort study determines predictors, course and consequences of depression and/or anxiety disorders in the Netherlands. Participants were healthy controls, persons with remitted depressive and/or anxiety disorders and persons with a current depressive and/or anxiety disorder. Participants were recruited from primary care $(n=1610)$, secondary care $(n=807)$ and from the general population $(n=564)$. General exclusion criteria of NESDA were a primary diagnosis of psychotic, obsessive compulsive, bipolar 
or severe addiction disorder. Participants who were not fluent in Dutch were also excluded. The NESDA study protocol was centrally approved by an Institutional Review Board and locally by the review boards of all participating sites and all participants signed written informed consent. A more detailed description of the NESDA study is available elsewhere (Penninx et al., 2008).

The current study used data from the baseline assessment, the one year follow-up assessment (FU1) and the two-year follow-up assessment (FU2). Psychopharmaca use was assessed at baseline at FU1 and at FU2 and coded according to the Anatomical Therapeutic Chemical (ATC) classification system (WHO, 2007). Patients who took any of the following medications when entering the study or during the study period were excluded from the analyses: antipsychotics (ATC code: N05A), anxiolytics (ATC code: N05B), hypnotics or sedatives (ATC code: N05C), psychostimulants (ATC code: N06B), or anti-dementia drugs (ATC code: N06D) Patients taking any of these drugs during the study period were excluded from the analyses because we aimed to study change in personality in untreated and treated persons receiving treatment for depression and not for anxiety or other disorders. Of the overall NESDA sample of 2981 participants, 1115 (37.4\%) had a diagnosis of current MDD (6-month recency) at baseline. Of these 1115 patients, 393 (35\%) were excluded from the analyses because they reported to have used one of the drugs above in the time period under study. Therefore, 722 patients with MDD were further considered for this study, of whom 13 persons had missing follow-up data, leading to an ultimate 709 persons with MDD as the final study sample.

\section{Treatment status}

Treatment status was defined by the use of antidepressant medication (ADM) and by self-reported contact with a psychologist or psychiatrist at any time between baseline and FU2. Patients who received treatment (psychological or medication) at baseline were also classified as treated. ADM use was assessed by self-report questionnaires and by inspection of drug containers that participants brought to the baseline and 2-year FU interview and classified according to the World Health Organization ATC classification system (WHO, 2007). Psychological treatment was assessed by the Trimbos/iMTA questionnaire for costs associated with psychiatric illness (TIC-P; Hakkaart-van Roijen, 2002) and defined as self-reported contact with a psychologist, psychiatrist or contact with a secondary care facility. To identify participants as treated no restrictions were placed on the number of contacts with a psychological health care provider. Based on these criteria, 520 (73.3\%) people were classified as treated and 189 (26.7\%) were classified as untreated.

\section{Measures}

CIDI. The Composite International Diagnostic Interview (CIDI) is a fully structured interview based on the Diagnostic and Statistical Manual of Mental Disorders (4th ed; DSM-IV; American Psychiatric Association, 1994) criteria (World Health Organization, 
1997). Excellent inter-rater reliability and validity has been reported for the CIDI (Andrews and Peters, 1998; Wittchen, 1994). In the NESDA study, the CIDI was used to determine the presence of current and lifetime mood disorders, anxiety disorders and alcohol abuse or dependence. The CIDI was conducted at baseline and at FU2.

IDS-SR. The Inventory of Depressive Symptoms Self-Report version (IDS-SR; Rush et al., 1996) is a 30-item self-report questionnaire measuring depressive symptom severity over the past seven days. Excellent internal consistency (Cronbach's alpha .92) and high correlations with the Hamilton Rating Scale for Depression (Hamilton, 1960) have been reported (Rush et al., 2003). In the current study the IDS-SR was obtained at baseline, FU1, and at FU2.

NEO-FFI. The revised NEO Five-Factor Inventory (NEO-FFI) is a widely used self-report instrument designed to measure the following higher order personality traits: neuroticism, extraversion, openness to experience, agreeableness, and conscientiousness (Costa and McCrae, 1995). Good internal consistency (Cronbach's alpha range .87 - .92) has been reported for the domain scales (Costa and McCrae, 1992). Although in the NESDA study all five subscales of the NEO-FFI have been assessed, the present report focuses on the neuroticism and extraversion scales because these two scales have most consistently been associated with depression. In the current study, the NEO-FFI was obtained at baseline and at FU2.

\section{Statistical analyses}

SPSS version 19 for Windows was used for statistical analyses. First, we computed Pearson correlations between depressive symptom severity and personality dimensions at baseline to determine concurrent relations. Pearson correlations between neuroticism at baseline and neuroticism at FU2 and between extraversion at baseline and extraversion at FU2 in treated and untreated persons were computed as an index of stability of these personality traits over time.

Multilevel Modeling (MLM) was used to predict change in depressive symptom severity and change in neuroticism and extraversion over time as a function of treatment status. MLM of longitudinal data has several advantages above ordinary least square regression analyses because it can deal with missing data and include time varying covariates in the analyses. Model building was guided by the following analytic procedure: First we added time as a predictor to the model (Model 1). In this model a significant main effect of time indicates that the dependent variables changes across the various assessment moments. Next we added treatment status and the interaction between treatment status and time as predictors (Model 2). In this model a significant time $\mathrm{x}$ treatment status interaction indicates that change in the dependent variable over time is different for treated and untreated persons. Following this we added personality scores as predictors to the model of change in depression severity and we added depression severity scores as predictors to the models of change in neuroticism and 
extraversion (Model 3). Finally we added the potential confounding variables gender, age, level of education and recruitment setting (Final Model). To compare the fit between the various models we compared change in deviance using a $\mathrm{X}^{2}$ test with 1 degree of freedom.

We also determined the relation between change in personality and change in depression severity in treated and untreated persons. To test whether change in personality predicts change in depressive symptom severity we added the neuroticism (at baseline and FU2) x time interaction and the extraversion (at baseline and FU2) $\mathrm{x}$ time interaction as predictors to the model of change in depressive symptom severity. To test whether the relation between change in personality and change in depressive symptom severity depends on treatment status, we also added the neuroticism (at baseline and FU2) $\mathrm{x}$ time $\mathrm{x}$ treatment status and the extraversion $\mathrm{x}$ time $\mathrm{x}$ treatment status three-way interactions to the model predicting change in depressive symptom severity. In these analyses neuroticism and extraversion were included as time varying covariates.

To test whether change in depressive symptom severity predicts change in personality, we conducted two separate MLM analyses, one with change in neuroticism as dependent variable and one with change in extraversion as dependent variable. To determine whether change in depressive symptoms predicts change in personality we entered the depressive symptoms (at baseline and FU2) x time interaction and the depressive symptoms (at baseline and FU2) $\mathrm{x}$ time $\mathrm{x}$ treatment status three-way interaction as predictor variables in both analyses. In these models, depressive symptoms severity was entered as time varying covariate.

In all models, continuous independent predictors were standardized by subtracting the sample mean from the individual score and dividing the result by the standard deviation of the mean to facilitate the interpretability of various predictors that are measured on different scales. In all MLMs we used an unstructured covariance structure for repeated measures over time and a Maximum Likelihood estimation method in order to be able to compare model fit. We computed effects sizes (Cohens'd) for all models based on estimates from the multi-level analyses (Feingold, 2009). Time was coded as follows: $0=$ baseline, $1=$ FU1, $2=$ FU2. Treatment seeking was centered and coded $-0.5=$ untreated and $0.5=$ treated.

\section{Results}

\section{Sample characteristics}

Demographic and clinical characteristics of the treated and the untreated group are shown in Table 1. Depressive symptom severity at baseline was significantly higher in the treated group $(M=31.46, S D=11.92)$ compared to the untreated group $(M=28.91$, $\mathrm{SD}=11.47)$. Baseline levels of extraversion were significantly higher in the untreated group ( $\mathrm{M}=36.87, \mathrm{SD}=7.16)$ compared to the treated group $(\mathrm{M}=34.88, \mathrm{SD}=6.99)$. It should be noted that these statistical differences are not necessarily clinically 
meaningful given that in a large sample also small mean differences can reach statistical significance. To estimate the clinical meaningfulness of these differences we also compute the effect size (Cohens' $d$ ) of the differences between the groups. The effect size of the difference between the groups was 0.22 for depression severity and 0.28 for extraversion, reflecting small effects (Cohen, 1988). The two groups also differed with respect to source of recruitment. The majority of people in the treated group were recruited from secondary care facilities (60.4\%), followed by recruitment from primary care facilities (33.3\%) and by recruitment from the general population (6.3\%). Most people from the untreated group were recruited from primary care facilities $(58.2 \%)$ followed by recruitment from secondary care facilities (26.5\%) and by recruitment from the general population (15.3\%). Gender was equally distributed among the two groups.

Table 1. Demographic and clinical characteristics at baseline for treated and untreated persons.

\begin{tabular}{|c|c|c|c|c|c|c|}
\hline \multirow[t]{2}{*}{ Characteristic } & \multicolumn{2}{|c|}{ Treated $(n=520)$} & \multicolumn{2}{|c|}{ Untreated ( $n=189)$} & \multirow[b]{2}{*}{$t$} & \multirow[b]{2}{*}{$\begin{array}{l}\text { Chi- } \\
\text { Square }\end{array}$} \\
\hline & Mean $(S D)$ & $N(\%)$ & Mean $(S D)$ & $N(\%)$ & & \\
\hline Age (years) & $39.95(11.69)$ & - & $38.80(12.77)$ & - & -1.16 & - \\
\hline Female & - & 337 (64.1) & - & $138(70.4)$ & - & 2.56 \\
\hline Education level (years) & $11.86(3.27)$ & - & $11.70(3.16)$ & - & -0.57 & - \\
\hline $\begin{array}{l}\text { Number of previous } \\
\text { episodes }^{8}\end{array}$ & $5.21(10.13)$ & - & $6.16(11.63)$ & - & 1.04 & - \\
\hline Recurrent MDD & - & $280(53.2)$ & - & $113(57.7)$ & - & 1.13 \\
\hline Anxiety disorder & - & $330(62.7)$ & - & $114(58.2)$ & - & 1.26 \\
\hline $\begin{array}{l}\text { Depressive symptom } \\
\text { severity }\end{array}$ & $31.46(11.92)$ & - & $28.91(11.47)$ & - & $-2.53^{*}$ & - \\
\hline Neuroticism & $42.08(6.59)$ & - & $41.05(6.84)$ & - & -1.81 & - \\
\hline Extraversion & 34.88 (6.99) & - & $36.87(7.16)$ & - & $2.72^{* *}$ & - \\
\hline
\end{tabular}

Note. ${ }^{*}=p<.05 ;^{* *}=p<.01$.

Concurrent relations between depressive symptom severity and the personality dimensions neuroticism and extraversion

At baseline, neuroticism correlated positively with depressive symptom severity $(r=.56$; $p<.001)$ and negatively with extraversion $(r=-.50 ; p<.001)$; extraversion correlated negatively with depressive symptom severity $(r=-.45 ; p<.001)$.

\footnotetext{
${ }^{8}$ Median number of previous episodes was two in the overall sample, in the treatment seeking group and in the non treatment seeking group.
} 


\section{Change in depressive symptom severity}

First, we modeled change in depressive symptom severity over time by treatment status using MLM. Table 2 summarizes the results of the four most informative models. In all models there was a significant main effect of time $(p<.01)$ indicating that depressive symptoms decreased over time $(d=0.89)$. In the final model, there was a significant main effect of time $(F(1,547.75)=216.83, p<.001)$, after adjusting for neuroticism and extraversion levels and other potential confounding variables. The interaction between time and treatment status was not significant $(F(1,547.74)=3.39, p=.07)$, suggesting that decrease in depressive symptoms did not depend on treatment status (Figure $1 \mathrm{~A}$ ).

Table 2. Predicting change in depressive symptom severity over time.

\begin{tabular}{lllll}
\hline Parameter & Model 1 & Model 2 & Model 39 & Final Model ${ }^{10}$ \\
\hline Intercept & $28.74(0.49)^{* *}$ & $27.58(0.59)^{* *}$ & $28.45(0.50)^{* *}$ & $29.32(1.08)^{* *}$ \\
Time & $-4.35(0.23)^{* *}$ & $-4.06(0.28)^{* *}$ & $-4.06(0.28)^{* *}$ & $-4.06(0.28)^{* *}$ \\
Treatment status & - & $3.92(1.18)^{* *}$ & $1.71(0.99)$ & $2.06(0.98)^{*}$ \\
Time*Treatment & - & $-1.00(0.55)$ & $-1.01(0.55)$ & $-1.02(0.55)$ \\
status & & & & 11299.817 \\
$-2 * \log$ likelihood & 11693.205 & 11682.059 & 11335.160 & $35.34^{* *}$ \\
$\Delta$ deviance & - & $11.14^{* *}$ & $346.90^{* *}$ & \\
\hline
\end{tabular}

Note. ${ }^{* *} p<.01 ; * p<.05$; All continuous predictor variables were standardized before entered into the model.

\section{Stability of neuroticism and extraversion}

To determine the stability of the personality dimensions neuroticism and extraversion over time in the two groups, we first determined Pearson correlations between neuroticism and extraversion at baseline and neuroticism and extraversion at FU2. In both groups, neuroticism at baseline correlated moderately and statistically significant with neuroticism levels at FU 2 (untreated: $r=.64, p<.001$; treated $r=.59, p<.001$ ) and the correlation coefficients of the two groups did not differ statistically significantly, $z=$ $0.78, p=.43$. Extraversion at baseline also correlated moderately and statistically significant with extraversion at FU2 in both groups (untreated: $r=.77, p<.001$; treated: $r=.72, p<.001$ ) and the correlation coefficients did not differ between groups, $z=1.10$, $p=.27$. The overall stability coefficient between extraversion at baseline and extraversion at FU2 $(r=.74)$ was stronger than the stability coefficient between neuroticism at baseline and neuroticism at FU2 $(r=.60 ; z=3.99, p<.001)$.

\footnotetext{
${ }^{9}$ Adjusted for neuroticism and extraversion at baseline and at FU2

${ }^{10}$ Adjusted for potential confounding variables: gender, age, level of education and recruitment setting
} 


\section{Mean level stability of neuroticism and extraversion}

Both groups experienced a statistically significant decrease in mean neuroticism levels from baseline to FU2 (mean decrease untreated: $-4.23, p<.001$; mean decrease treated: -3.68, $p<.001$ ) and a statistically significant increase in the mean level of extraversion (mean increase untreated: $1.67 ; p<.001$; mean increase treated: $1.68 ; p<.001$ ). To compare the mean level change in neuroticism and extraversion in the current sample with a non-depressed control group, we also calculated the mean level stability of persons in the same study (Penninx et al., 2008) without any depressive or anxiety disorders $(\mathrm{N}=652)$. Mean neuroticism levels in the non-depressed group also decreased statistically significantly from baseline to FU2 (mean decrease: $-1.44, p<.001$ ) as did the mean level of extraversion (mean decrease: -1.32; $p<.001$ ). The mean decrease in neuroticism in the non-depressed group statistically significantly differed from the mean decreased in the untreated $(t(714)=3.50, p<.001)$ and the treated group $(t(1032)$ $=4.74, p<.001$ ). The mean difference in extraversion in the non-depressed group also statistically significantly differed from the mean difference in extraversion in the untreated $(t(714)=3.18, p<.01)$ and treated group $(t(1032)=5.79, p<.001)$.

Next we modeled change in neuroticism and extraversion over time as a function of treatment status using MLM (Table 3). In all models, predicting change in neuroticism over time there was a significant main effect of time $(p<.01)$, indicating that neuroticism decreased over time $(d=0.73)$. In the final model the significant main effect of time remained after controlling for depressive symptom severity and extraversion levels and for potential confounding variables $F(1,474.05)=92.45, p<.001$. The interaction between time and treatment status was not significant $(F(1,464.61)=0.40, p$ $=.53$ ), indicating that decrease in neuroticism did not differ between treated and untreated persons (Figure $1 \mathrm{~B}$ ).

In the initial model predicting change in extraversion over time there was a significant main effect of time $(p<.01)$ indicating that extraversion increased over time, however, after controlling for neuroticism and depression severity at baseline and follow-up change in extraversion over time was not significant anymore $(F(1,512.51)=0.01$, $p=.91)$. The interaction between time and treatment status was also not significant $(F(1$, $478.23)=2.13, p=.15)$, indicating that extraversion remained stable over time in both treated and untreated persons (Figure $1 \mathrm{C}$ ).

\section{Differences in change in depression severity and personality between different types of treatment}

To test whether change in depressive symptom severity, neuroticism or extraversion differed between different types of treatment (medication, psychotherapy, or both medication and psychotherapy) we added dummy coded variables for the different types of treatment to the final multilevel models. In these analyses the untreated group was used as the reference condition. There were no differences between type of treatment and change in depressive symptom severity, change in neuroticism or change in extraversion (all $p$-values $>.05$ ). 
Relation between change in personality and change in depression severity

In the model predicting change in depressive symptom severity over time, the neuroticism $\mathrm{x}$ time $\mathrm{x}$ treatment status and the extraversion $\mathrm{x}$ time $\mathrm{x}$ treatment status three-way interactions were not significant $(F(1,550.42)=0.48, p=.49$ and $F(1,549.68)$ $=1.67, p=.20$, respectively). Therefore, we removed the non-significant three-way interactions from the model and the model was rerun. The interactions between time and neuroticism and the interaction between time and extraversion were not significant $(F(1,734.65)=0.22, p=.64$ and $F(1,667.54)=0.15, p=.70$, respectively $)$, indicating that change in personality over time did not predict change in depressive symptom severity over time.

In the model predicting change in neuroticism over time the depressive symptom severity $\mathrm{x}$ time $\mathrm{x}$ treatment status interaction was not significant $(F(1,549.64)=0.20, p$ $=.66$ ) and was therefore removed from the model. There was a significant interaction between depressive symptom severity and time, $F(1,683.96)=25.89, p<.001$. To interpret the interaction term, we plotted the neuroticism total scores at baseline and at FU2 separately for the low- and high change in depressive symptom severity groups (Figure 2), defined as one $S D$ below and above the mean change in depressive symptom severity. Patients in the high change group $(n=82)$ reported a mean decrease of 27.70 $(S D=6.04)$ on the IDS whereas patients in the low change group $(n=76)$ reported a mean increase of $5.93(S D=3.63)$ on the IDS. As can be seen in Figure 2, persons in the high decrease in depressive symptoms group significantly decreased on neuroticism from baseline to FU2 whereas increased depressive symptoms were unrelated to changes in neuroticism from baseline to FU2 (ES within decrease in depressive symptoms group $d=1.91$; ES within increased depressive symptoms group: $d=0.06$ ). In the model predicting change in extraversion over time the depressive symptom severity $\mathrm{x}$ time $\mathrm{x}$ treatment status interaction was not significant $(F(1,546.24)=0.99, p=.32)$ and was therefore removed from the model. After removing the non-significant threeway interaction from the model, the interaction between depressive symptom severity and time was not significant, $F(1,650.31)=1.69, p=.19$, indicating that change in depressive symptoms did not predict change in extraversion. 
Table 3. Predicting change in the personality dimensions neuroticism and extraversion over time.

\begin{tabular}{|c|c|c|c|c|}
\hline \multirow[b]{2}{*}{ Parameter } & \multicolumn{4}{|l|}{ Neuroticism } \\
\hline & Model 1 & Model 2 & Model $3^{11}$ & Final Model ${ }^{12}$ \\
\hline Intercept & $41.27(0.30)^{* *}$ & $40.82(0.38)^{* *}$ & $41.24(0.30)^{* *}$ & $41.06(0.68)^{* *}$ \\
\hline Time & $-3.65(0.30)^{* *}$ & $-3.71(0.37)^{* *}$ & $-3.21(0.33)^{* *}$ & $-3.18(0.33)^{* *}$ \\
\hline Treatment status & - & $1.51(0.75)^{*}$ & $-0.03(0.60)$ & $-0.08(0.58)$ \\
\hline Time*Treatment status & - & $0.20(0.73)$ & $0.40(0.66)$ & $0.42(0.66)$ \\
\hline$-2 * \log$ likelihood & 6250.052 & 6245.145 & 5854.975 & 5795.704 \\
\hline \multirow[t]{2}{*}{$\Delta$ deviance } & - & $4.907^{*}$ & $390.17^{* *}$ & $59.271^{* *}$ \\
\hline & \multicolumn{4}{|l|}{ Extraversion } \\
\hline Parameter & Model 1 & Model 2 & Model $3^{13}$ & Final Model ${ }^{14}$ \\
\hline Intercept & $33.80(0.32)^{* *}$ & $34.43(0.40)^{* *}$ & $34.44(0.34)^{* *}$ & $34.87(0.79)^{* *}$ \\
\hline Time & $1.53(0.23)^{* *}$ & $1.36(0.29)^{* *}$ & $0.03(0.27)$ & $-0.05(0.27)$ \\
\hline Treatment status & - & $-2.13(0.79)^{* *}$ & $-0.98(0.67)$ & $-0.98(0.66)$ \\
\hline Time*Treatment status & - & $0.57(0.57)$ & $0.94(0.50)$ & $0.64(0.50)$ \\
\hline$-2 * \log$ likelihood & 6054.320 & 6047.172 & 5735.104 & 5693.581 \\
\hline$\Delta$ deviance & - & $7.15^{* *}$ & $312.07 * *$ & $41.25^{* *}$ \\
\hline
\end{tabular}

Note. ${ }^{* *}=p<.01,{ }^{*} p<.05$; All continuous predictor variables were standardized before entered into the model.

\section{Discussion}

The aim of this study was to determine the two-year stability of neuroticism and extraversion in treated and untreated persons with major depressive disorder (MDD) in the general population. We determined the stability of depressive symptom severity, neuroticism and extraversion over a period of two years in depressed patients who sought treatment for mental health problems during the study period and in depressed persons who did not. Depressive symptom severity and neuroticism scores significantly decreased statistically during the study period regardless of treatment status. These findings are in line with previous findings from the NESDA study showing that depressive symptoms decrease over time regardless of treatment status (Penninx et al., 2011) and with findings from another cohort study in the Netherlands showing that treated depressed patients in primary care have similar outcomes than unrecognized depressed patients not receiving treatment (Kamphuis et al., 2011). Contrary to our findings an earlier cohort study found that treated depressed patients have better outcomes than untreated persons with depression (Angst, 1998) whereas in another

\footnotetext{
11 Adjusted for depressive symptom severity at all time points and for extraversion at baseline and FU2

12 Adjusted for potential confounding variables: gender, age, level of education, recruitment setting

${ }^{13}$ Adjusted for depressive symptom severity at all time points and for neuroticism at baseline and FU2

${ }^{14}$ Adjusted for potential confounding variables: gender, age, level of education, recruitment setting
} 
population based study it has been shown that treated depressed patients had worse outcome than untreated persons with depression (Wang, 2004). It should be noted that our finding that treatment status was not related to change in depression, neuroticism and extraversion over time does not suggest that receiving treatment does not impact these variables. Treated and untreated persons in this study were not randomized and the effect of a given treatment on change in depression severity and personality traits can only be determined in randomized trials because observational studies suffer from confounding by indication when comparing treated and non-treated patients.

It has been shown previously, in the context of RCTs, that depression treatment is related to decreases in neuroticism (Ekselius and von Knorring, 1999; Hellerstein et al., 2000; Tang et al., 2009). However, previous studies determined the short-term stability of neuroticism over a course of depression treatment with SSRIs whereas the current study determined the long term (two-year) stability of neuroticism and extraversion in treated and untreated persons with depression in the general population. Another explanation for the discrepant findings, as suggested by one of the anonymous reviewers, is that the current study did not differentiate between specific types of antidepressants whereas previous RCTs focused specifically on SSRIs. It is possible that SSRIs have stronger effects on changes in neuroticism than other types of antidepressants. Determining the specific impact of different types of antidepressants on changes in neuroticism and other personality traits requires randomization to different types of antidepressants and is therefore an issue for future RCT based studies.

We also determined relations between change in depressive symptom severity and change in personality in treated and untreated persons with depression and found that change in depression was associated with change in neuroticism independently of treatment status. Patients experiencing strong symptom reduction reported more decrease in neuroticism over time compared to patients experiencing an increase in depressive symptoms over time (Figure 2). Moreover, changes in neuroticism and extraversion were not associated with changes in depressive symptoms. This finding suggests that changes in depressive state might drive changes in the underlying personality rather than the other way around, which is in line with findings showing that neuroticism and extraversion in depressed patients are mood-state dependent (Karsten et al., 2012). It should be noted, however, that the current research design and analytic approach does not allow drawing any causal conclusions. Identifying the causal directions of the relation between changes in personality and depression is thus an issue for future research.

\section{Limitations}

The findings of this study should be interpreted in the light of several limitations. First, patients in this study were not randomized to treatment conditions and might therefore not be directly comparable. Persons in the treated and in the untreated group differed with respect to severity of depressive symptoms and extraversion scores at baseline. Therefore any differences between the two groups cannot be solely attributed to 
treatment status. Second we did not assess treatment adequacy. It should be noted however that a previous NESDA study found that treatment adequacy was unrelated to improvement in depressive symptoms (Prins et al., 2011). Third, we homogenized the sample by excluding patients who took medication not directly related to depression (including anxiolytics) during the study period. This might limit the generalizability of our findings to depressed patients with more diverse symptoms. Fourth, we determined cross-sectional relations between change in depression levels and change in personality. Given that in the present analysis personality was only assessed at two time points we could not determine any temporal relations between change in depression severity and change in personality. Finally, we did not determine the stability of other personality dimensions in treated and untreated persons. It has been shown in a previous NESDA study that the personality dimension of conscientiousness also changes with depressive state (Karsten et al., 2012).

\section{Implications}

Despite these limitations the results of this study have several implications for clinical settings and future research. We found that in a large sample of patients with MDD, treatment status was unrelated to change in neuroticism and extraversion. We also found that change in depression predicted change in neuroticism rather than the other way around. These findings suggest that changes in neuroticism might be driven by improved depressive state (the mood-state effect) rather than by receiving treatment for depression. From a clinical point of view this suggests that treatment for depression should focus on depressive symptoms rather than on the underlying personality structure because treating the underlying personality structure of depressed patients might not improve depressive symptom severity. Future research studying the stability of personality in the context of treatment should also take into account untreated persons with MDD who improve in depression severity in order to be able to distinguish mood-state effects from more direct effects of treatment on personality. Given that in our study the treated group differed from the untreated group with respect to several clinical and demographic variables it is not possible to draw any conclusions regarding the causal effect of treatment status on change in neuroticism and extraversion. Future research should determine characteristics of depressed persons in the general population who profit from seeking treatment for depression with respect to changes in neuroticism and extraversion. Finally, in line with what has previously been reported in the NESDA sample (Karsten et al., 2012) our finding that neuroticism changes with depressive state suggest that personality assessment is partly clouded by the current mood state. 


\section{References}

American Psychiatric Association, 1994. Diagnostic and Statistical manual of mental disorders (4th ed.). American Psychiatric Press, Washington, D.C.

Andrews, G., Peters, L., 1998. The psychometric properties of the Composite International Diagnostic Interview. Soc. Psychiatry Psychiatr. Epidemiol. 33, 80-88.

Angst, J., 1998. Treated versus untreated major depressive episodes. Psychopathology 31, 37-44.

Berlim, M.T., McGirr, A., Beaulieu, M.-M., Van den Eynde, F., Turecki, G., 2013. Are neuroticism and extraversion associated with the antidepressant effects of repetitive transcranial magnetic stimulation (rTMS)? An exploratory 4-week trial. Neurosci. Lett. 534, 306-310.

Boyce, P., Parker, G., Barnett, B., Cooney, M., 1991. Personality as a vulnerability factor to depression. Br. J. Psychiatry 159, 106-114.

Cohen, J., 1988. Statistical power analysis for the behavioral sciences. Erlbaum, Hillsdale, NJ.

Costa, P.T., McCrae, R.R., 1992. Revised NEO Personality Inventory and NEO Five-Factor Inventory: Professional Manual. Psychological Assessment Resources, Inc, Lutz, FL.

Costa, P.T., McCrae, R.R., 1995. Domains and Facets: Hierarchical Personality Assessment Using the Revised NEO Personality Inventory. J. Pers. Assess. 64, 21-50.

Ekselius, L., von Knorring, L., 1999. Changes in personality traits during treatment with sertraline or citalopram. Br. J. Psychiatry 174, 444-448.

Fanous, A.H., Neale, M.C., Aggen, S.H., Kendler, K.S., 2007. A longitudinal study of personality and major depression in a population-based sample of male twins. Psychol. Med. 37, 1163-1172.

Feingold, A., 2009. Effect sizes for growth-modeling analysis for controlled clinical trials in the same metric as for classical analysis. Psychol. Methods 14, 43-53.

Ferguson, C.J., 2010. A meta-analysis of normal and disordered personality across the life span. J. Pers. Soc. Psychol. 98, 659-667.

Hakkaart-van Roijen, L., 2002. Trimbos/iMTA questionnaire for costs associated with psychiatric illness (TIC-P). Institute for Medical Technology Assessment., Rotterdam.

Hamilton, M., 1960. A rating scale for depression. J. Neurol. Neurosurg. Psychiatry 23, 56-61.

Hellerstein, D.J., Kocsis, J.H., Chapman, D., Stewart, J.W., Harrison, W., 2000. Double-blind comparison of sertaline, imipramine, and placebo in the treatment of dysthymia: Effects on personality. The American Journal of Psychiatry 157, 1436-1444.

Hettema, J.M., Neale, M.C., Myers, J.M., Prescott, C.A., Kendler, K.S., 2006. A Population-Based Twin Study of the Relationship Between Neuroticism and Internalizing Disorders. Am. J. Psychiatry 163, 857-864.

Hirschfeld, R.M., Klerman, G.L., Lavori, P., Keller, M.B., 1989. Premorbid personality assessments of first onset of major depression. Arch. Gen. Psychiatry 46, 345-350.

Kamphuis, M.H., Stegenga, B.T., Zuithoff, N.P.A., King, M., Nazareth, I., de Wit, N.J., Geerlings, M.I., 2011. Recognition of depression in primary care: does it affect outcome? The PREDICT-NL study. Fam. Pract.

Karsten, J., Penninx, B.W.J.H., Riese, H., Ormel, J., Nolen, W.A., Hartman, C.A., 2012. The state effect of depressive and anxiety disorders on big five personality traits. J. Psychiatr. Res.

Kendler, K.S., Gatz, M., Gardner, C.O., Pedersen, N.L., 2006. Personality and Major Depression: A Swedish Longitudinal, Population-Based Twin Study. Arch. Gen. Psychiatry 63, 1113-1120.

Kendler, K.S., Kuhn, J., Prescott, C.A., 2004. The Interrelationship of Neuroticism, Sex, and Stressful Life Events in the Prediction of Episodes of Major Depression. Am. J. Psychiatry 161, 631-636.

Kessler, R.C., Berglund, P., Demler, O., Jin, R., Koretz, D., Merikangas, K.R., Rush, A.J., Walters, E.E., Wang, P.S., 2003. The Epidemiology of Major Depressive Disorder. JAMA: The Journal of the American Medical Association 289, 3095-3105.

Kotov, R., Gamez, W., Schmidt, F., Watson, D., 2010. Linking "big" personality traits to anxiety, depressive, and substance use disorders: A meta-analysis. Psychol. Bull. 136, 768-821.

Ormel, J., Oldehinkel, A.J., Vollebergh, W., 2004. Vulnerability Before, During, and After a Major Depressive Episode: A 3-Wave Population-Based Study. Arch. Gen. Psychiatry 61, 990-996. 
Penninx, B.W.J.H., Beekman, A.T.F., Johannes, H.S., Zitman, F.G., Nolen, W.A., Spinhoven, P., Cuijpers, P., de Jong, P.J., van Maruiijk, H.W.J., Assendelft, W.J.J., van der Meer, K., Verhaak, P., Wensing, M., de Graaf, R., Hoogendijk, W.J., Ormel, J., van Dyck, R., 2008. The Netherlands Study of Depression and Anxiety (NESDA): Rationale, objectives and methods. International Journal of Methods in Psychiatric Research 17, 121-140.

Penninx, B.W.J.H., Nolen, W.A., Lamers, F., Zitman, F.G., Smit, J.H., Spinhoven, P., Cuijpers, P., de Jong, P.J., van Marwijk, H.W.J., van der Meer, K., Verhaak, P., Laurant, M.G.H., de Graaf, R., Hoogendijk, W.J., van der Wee, N., Ormel, J., van Dyck, R., Beekman, A.T.F., 2011. Two-year course of depressive and anxiety disorders: Results from the Netherlands Study of Depression and Anxiety (NESDA). J. Affect. Disord. 133, 76-85.

Prins, M., Verhaak, P., Hilbink-Smolders, M., Spreeuwenberg, P., Laurant, M., van der Meer, K., van Marwijk, H., Penninx, B., Bensing, J., 2011. Outcomes for depression and anxiety in primary care and details of treatment: a naturalistic longitudinal study. BMC Psychiatry 11.

Rush, A.J., Gullion, C.M., Basco, M.R., Jarrett, R.B., Trivedi, M.H., 1996. The Inventory of Depressive Symptomatology (IDS): psychometric properties. Psychol. Med. 26, 477-486.

Rush, A.J., Trivedi, M.H., Ibrahim, H.M., Carmody, T.J., Arnow, B., Klein, D.N., Markowitz, J.C., Ninan, P.T., Kornstein, S., Manber, R., Thase, M.E., Kocsis, J.H., Keller, M.B., 2003. The 16-item Quick Inventory of Depressive Symptomatology (QIDS), clinician rating (QIDS-C), and self-report (QIDS-SR): A psychometric evaluation in patients with chronic major depression. Biol. Psychiatry 54, 573-583.

Tang, T.Z., DeRubeis, R.J., Hollon, S.D., Amsterdam, J., Shelton, R., Schalet, B., 2009. Personality change during depression treatment: A placebo-controlled trial. Arch. Gen. Psychiatry 66, 1322-1330.

Wang, J., 2004. A Longitudinal Population-Based Study of Treated and Untreated Major Depression. Med. Care 42, 543-550.

WHO, 2007. Anatomical Therapeutic Chemical (ATC) Classification. World Helath Organization, Geneva, Switzerland.

Wittchen, H.-U., 1994. Reliability and validity studies of the WHO-Composite International Diagnostic Interview (CIDI): A critical review. J. Psychiatr. Res. 28, 57-84.

World Health Organization, 1997. Composite International Diagnostic Interview (CIDI). World Health Organization, Geneva.

Young, A.S., Klap, R., Sherbourne, C.D., Wells, K.B., 2001. The Quality of Care for Depressive and Anxiety Disorders in the United States. Arch. Gen. Psychiatry 58, 55-61. 


\section{CHAPTER 6 \\ Stability of interpersonal problems during cognitive therapy for depression}

Published as:

Renner, F., Jarrett, R. B., Vittengl, J. R., Barrett, M. S., Clark, L. A., \& Thase, M. E. (2012). Interpersonal problems as predictors of therapeutic alliance and symptom improvement in cognitive therapy for depression. Journal of Affective Disorders, 138(3), 458-467. 


\section{Abstract}

Background: The degree to which interpersonal problems of depressed patients improve over the course of cognitive therapy (CT) and relate to the quality of the therapeutic alliance and to symptom improvement, remain unclear.

Methods: We analyzed data of adult outpatients $(N=523)$ with major depressive disorder participating in a clinical trial to determine the factor structure of the Inventory of Interpersonal Problems-Circumplex (IIP-C) and to relate the observed factor scores to the quality of the therapeutic alliance and symptom improvement over the course of CT. Patients received 16-20 sessions protocol (50-60 minutes each) of individual CT according to the treatment manual by Beck et al. (1979).

Results: We found a three-factor structure (interpersonal distress, agency, and communion) of interpersonal problems. Interpersonal distress decreased $(d=.90)$, but interpersonal style did not change substantively during CT (communion $d=.03$; agency $d=.14)$. High initial agency scores related negatively to the therapeutic alliance $(\beta=$ .12), whereas high initial communion scores related positively to the therapeutic alliance $(\beta=.15)$. Elevated pre-treatment interpersonal distress scores were related to both weaker therapeutic alliances $(\beta=.13)$ and higher symptom levels throughout treatment $(\beta=.10)$. Limitations: All patients in this study had recurrent MDD and it is therefore uncertain whether the results would generalize to patients with other psychiatric disorders.

Conclusions: This study supports the use of the IIP-C as a comprehensive measure of patients' interpersonal style and interpersonal distress. The IIP-C measured before CT showed some predictive validity with respect to therapeutic alliance measured at the midpoint and therapy outcome. The clinical importance of these findings is discussed.

Keywords: Recurrent Depression, Cognitive Therapy, Personality, Interpersonal Style, Therapeutic Alliance 


\section{Introduction}

Interpersonal problems refer to persistent difficulties that individuals experience in their social relationships (Horowitz et al., 1988; Horowitz et al., 1993). Patients seeking psychotherapy usually report some degree of such problems in addition to their primary symptoms (Horowitz et al., 1988). Moreover, patients with major depressive disorder (MDD) are significantly more distressed by their interpersonal problems than nondepressed individuals (Barrett and Barber, 2007). The broad aim of the present study was to document further the range of interpersonal problems in depressed patients, to determine the extent to which these problems change over a 12-14 week protocol of cognitive therapy (CT; Beck et al., 1979), and to examine relations among interpersonal problems, the therapeutic alliance, and therapy outcome.

Recent efforts to conceptualize interpersonal problems in patients with various clinical problems have relied primarily on the interpersonal circumplex, in which stable interpersonal dispositions are mapped along a two-dimensional space ranging from agency to submission on one axis and from communion to coldness on the other (Horowitz et al., 2006; Wiggins, 1982) ${ }^{15}$. Agency refers to persons' influence over others and includes traits such as autonomy, independence, and dominance, whereas communion describes involvement with others and includes traits such as cooperation and caring. One of the most frequently used assessment tools in this context is the Inventory of Interpersonal Problems-Circumplex version (IIP-C; Alden et al., 1990; Horowitz et al., 1988).

Previous research has investigated the stability of the IIP-C. Vittengl et al. (2003), for example, assessed interpersonal problems before and after CT in outpatients with recurrent MDD, and found that general distress decreased substantially following 20 sessions of CT, whereas agency and communion scores remained unchanged. From these findings, the authors concluded that the agency and communion dimensions of the IIP-C represent stable interpersonal styles, whereas general distress represents a statelike aspect of interpersonal functioning. In an attempt to replicate these findings, Holtforth et al. (2006) administered the IIP-C to a group of psychiatric outpatients with primarily anxiety and affective disorders, before and after an average of 29 sessions of eclectic psychotherapy. In contrast to the findings by Vittengl et al. (2003), Holtforth and colleagues found that both general distress and interpersonal style (agency and communion) changed during therapy, although distress changed more. Given the inconsistent findings, there is a need to investigate further the (in)stability of general distress and interpersonal style over the course of different types of psychotherapy.

Another relevant question is to what extent interpersonal style and distress relate to the therapeutic alliance and therapy outcome. Interpersonal theories of psychotherapy suggest that patients' friendly-submissive behaviors are complementary

\footnotetext{
${ }^{15}$ In the circumplex literature, the $\mathrm{x}$-axis of the circumplex usually was referred to as love, warmth or nurturance and the $y$-axis as dominance, power, or status. More recently, however, the superordinate terms communion and agency have been used for the $\mathrm{x}$-axis and $\mathrm{y}$-axis, respectively.
} 
to therapists' friendly-dominant behaviors (Kiesler, 1983; Kiesler and Watkins, 1989; Tracey, 1993). Accordingly, patients' having a friendly-submissive interpersonal style should facilitate the therapeutic relationship and positively affect therapy outcome. In contrast, hostile-dominant behaviors likely would conflict with therapy goals, thus contributing to a poor therapeutic alliance and treatment outcome (Tracey, 1993). In terms of IIP-C structure, theory suggests that high communion and low agency are associated with better therapeutic relationships and outcomes. Consistent with this, most research to date indicates that in naturalistic settings, outpatients scoring high on the communion dimension show more symptom improvement over the course of shortterm psychodynamic psychotherapy (Filak et al., 1986; Gurtman, 1996; Schauenburg et al., 2000).

Findings regarding the role of agency in predicting therapy outcome and symptom reduction over time are mixed. Some (Borkovec et al., 2002; Gurtman, 1996), but not all (Filak et al., 1986; Schauenburg et al., 2000) studies report an association between high agency scores and poor treatment outcome. Moreover, Ruiz et al. (2004) found that neither communion nor agency was associated with treatment outcome after controlling for general distress. Thus, although most studies support the influence of interpersonal style on therapy outcome, there are also contradictory findings and indications that outcomes are better accounted for by general distress.

Studies relating interpersonal style and distress to the quality of the therapeutic alliance found that interpersonal problems in the hostile-dominant domain (i.e., high agency, low communion) prior to treatment predicted a poor working alliance (Connolly Gibbons et al., 2003; Muran et al., 1994; Puschner et al., 2005). Moreover, IIP-C general distress factor has been associated with poor working alliance (Constantino and SmithHansen, 2008), whereas interpersonal problems in the friendly-submissive domain (i.e., low agency, high communion) have been associated with better alliance (Constantino and Smith-Hansen, 2008; Muran et al., 1994; Puschner et al., 2005). In contrast, Paivio \& Bahr (1998) found that the IIP-C octant scales social avoidance and non-assertiveness, reflecting low agency and high communion, related negatively to working alliance.

Previous research relating the IIP-C to therapy outcome and the therapeutic alliance either categorized patients' reports into interpersonal problem areas based on the predominant interpersonal problem theme, or used IIP-C parameters to predict outcome or therapeutic alliance. The computation of IIP-C parameters is based on octant scores, which all mark a general distress factor that correlates with anxiety and depression symptoms (Horowitz et al., 2000). Accordingly, a clear distinction between interpersonal style and general distress cannot be made, and some have argued that to identify unique effects of interpersonal style on outcome, the general distress factor should be controlled before examining the octant scales (Vittengl et al., 2003).

One possibility for controlling general distress is to obtain orthogonal factor scores from IIP-C scales that converge highly with the underlying IIP-C structure (Vittengl et al., 2003). This can be done by factor analyzing the IIP-C scales, thereby creating independent factor scores representing both interpersonal style and general distress. Identification of orthogonal factor scores simplifies the interpretation of interpersonal 
distress distinct from interpersonal style, as captured by the communion and affiliation factors. Vittengl et al. (2003), for example, applied principal components analysis to IIP octant scales and found a three-factor structure resembling the three IIP-C dimensions general distress, agency, and communion. Accordingly, to address the clinically important issues of stability and change of interpersonal style and distress, as well as the relations of these constructs with therapy outcome and the therapeutic alliance, factor scores extracted from the IIP-C scales are preferred over octant scales. Yet, to the best of our knowledge, no published study has addressed the relation of patient's initial interpersonal style and distress with therapy outcome and the therapeutic alliance using orthogonal factor scores as predictors.

Therefore, we sought first to replicate previous findings demonstrating a stable IIP-C circumplex structure (Alden et al., 1990; Horowitz et al., 2000; Vittengl et al., 2003). Although the IIP-C circumplex structure has been demonstrated in depressed patients (Vittengl et al., 2003), the number of outpatients in the present study is about four times larger, thus providing a more reliable estimate of the underlying factor structure. We hypothesized that (1) the IIP-C would demonstrate a three-factor structure, consisting of general distress, agency, and communion factors, both pre- and post-CT. Second, we examined interpersonal problems in depressed patients before and after CT in terms of general distress and angular placement in the circumplex, and hypothesized that (2) the interpersonal style of depressed patients would fall between non-assertiveness and social-avoidance. Third, we sought to determine whether the dimensions of communion and agency represent trait-like interpersonal styles, and hypothesized that (3) the distress factor would decrease from pre- to post-CT, whereas interpersonal style would remain stable.

Following this analysis, we assessed the clinical validity of the IIP-C in predicting therapeutic alliance and therapy outcome and hypothesized that (4) the distress and agency factors would relate negatively to improvement of depressive symptoms, whereas the communion factor would relate positively to symptom improvement. Finally, with respect to therapeutic alliance, we hypothesized that (5) distress and agency would relate negatively, and communion positively, to the quality of the therapeutic alliance.

\section{Methods}

\section{Sample}

The present report is based on patients participating in the acute phase of the 'Continuation Phase Cognitive Therapy Relapse Prevention (C-CT-RP) Trial' (ClinicalTrials.gov Identifiers: NCT00118404, NCT00183664 and NCT00218764). A more detailed description of this multi-stage study is available elsewhere (Jarrett and Thase, 2010). Study protocols were approved annually by the Institutional Review Boards at the two treatment sites (The University of Pittsburgh Medical Center and The University of Texas Southwestern Medical Center) and all 523 out-patients enrolled in 
the study provided written informed consent. The primary inclusion criteria for participation in the study were: a) a principal diagnosis of recurrent MDD according to the Diagnostic and Statistical Manual of Mental Disorders (4th ed; DSM-IV; American Psychiatric Association, 1994) criteria as assessed by the Structured Clinical Interview for DSM-IV (SCID-I; First et al., 1996) a history of inter-episode recovery with or without antecedent dysthymic disorder, c) a score of at least 14 on the 17-item version of the Hamilton Rating Scale for Depressive Symptoms (HRSD-17; Hamilton, 1960) and d) age between 18 and 70 years.

Patients were excluded if they had a poorly controlled medical disorder that might be correlated with depressed mood either directly or through medication intake; had a $D S M-I V$ diagnosis of any psychotic or organic mental disorder; were pregnant or planned to become pregnant within the next year; or had an active alcohol or drug dependence, bipolar disorder or predominant obsessive compulsive disorder, eating disorder or borderline personality disorder. Moreover, patients were excluded if they were unable to complete questionnaires in English, declined protocol requirements or previously had failed to respond to cognitive therapy or fluoxetine.

The average age of the sample entering the acute phase $(N=523)$ was 42.4 years $(S D=12.1) ; 67.5 \%$ were female; mean education level was 15.1 years $(S D=2.93) ; 32.5 \%$ were married, $30.2 \%$ single, $19.9 \%$ divorced, $5.7 \%$ separated, $2.3 \%$ widowed and $9.4 \%$ lived together with their partner. Patients' mean age of MDD onset was 21.2 years ( $S D=$ 10.8); median length of current episode was 10 months, mean 25.0 months $(S D=45.1)$. Mean pretreatment HRSD was 20.4 (range = 13-31; Two patients entered the protocol in error, with HRSD-17 scores of 13 at one of the two diagnostic visits; during cognitive therapy one responded and one dropped out.

\section{Procedure}

Acute-phase cognitive therapy. The acute-phase protocol consisted of 16 to 20 sessions, each lasting between 50 and $60 \mathrm{~min}$, across 12 to 14 weeks. Therapy was conducted by experienced, research-certified therapists according to the methods of Beck et al. (1979). The first eight sessions occurred twice weekly. Thereafter, patients who experienced at least a $40 \%$ reduction of HRSD scores were seen weekly, whereas those with less improvement received four additional weeks of twice weekly sessions; 410 completed at least 14 of 16 protocol sessions (early responders) or 18 of 20 protocol sessions (late responders).

\section{Assessments}

To assess changes in symptom severity, therapists completed the HRSD-17 (Hamilton, 1960) weekly during cognitive therapy, as did an independent evaluator at patient exit or end of treatment. Adequate internal consistency ( $r s=.46-.97)$, interrater reliability $(r s=.82-.98)$ and retest reliability $(r s=.81-.98)$ have been reported for the HRSD 
(Bagby et al., 2004). In the current study we found good interrater reliability (ICC = .91) for the HRSD in a multilevel analysis based on 28 patients rated by 4-14 clinicians each.

Inventory of Interpersonal Problems. The Inventory of Interpersonal Problems (IIP; Horowitz et al., 1988) is a 127-item self-report questionnaire assessing interpersonal difficulties with eight subscales (overly nurturant, intrusive, domineering, vindictive, cold, socially avoidant, nonassertive, exploitable). Each item is phrased as a statement and respondents rate their agreement on a Likert scale ranging from 1 (not at all) to 5 (extremely). In this study, responses to the IIP were scored according to the circumplex system, which is based on 64 items assessing interpersonal problems within two dimensions: agency and communion (IIP-C; Alden et al., 1990). Acceptable internal consistency (median $\alpha=.81$, range $.76-.88, N=800$ ) and test-retest reliability (median $r$ $=.73$, range $.56-.83, N=60$ ) of the IIP-C have been reported (Horowitz et al., 2000). In the present study internal consistency (coefficient alpha) for the overall score was .92 pre-treatment and .95 post-treatment. The IIP-C subscales were also shown to demonstrate moderate relations with the Beck Depression Inventory (median $r=.38$, range $=.33-.43, N=495$ ) and Beck Anxiety Inventory (median $r=.36$, range $=.31-.39, N$ = 495). The IIP-C was collected at the beginning, middle and end of the acute phase.

Working Alliance Inventory. The Working Alliance Inventory (WAI; Horvath and Greenberg, 1989) is a 36-item self-report measure of the therapeutic alliance, with therapist and client versions. Only the client form (WAI-C) was analyzed here because it has been shown to predict outcome better than the therapist version (Horvath and Symonds, 1991). The WAI has three subscales: task agreement, goal agreement and bond development. Each item is rated on a 7-point Likert-type scale. High internal consistency has been reported for the three subscales (.89-.92) and the overall scale (.87-.93; Horvath and Greenberg, 1989). The WAI-C has been shown to converge moderately with the California Psychotherapy Alliance Scales ( $r=.74$; Gaston, 1991) and the Penn Helping Alliance Scales ( $r=.85$; Alexander et al., 1986; Hatcher and Barends, 1996). The WAI-C was obtained at the middle and at the end of the acute phase. In the present study, internal consistency (coefficient alpha) was .94 for the overall WAI-C score at the middle of the acute phase.

\section{Statistical analyses}

Unless stated otherwise, SPSS version 18 for Windows was used for statistical analyses. To investigate hypothesis (1), a principal components analysis was conducted with planned rotations (Vittengl et al., 2003). Principal components analysis was used because the circumplex scales of the IIP were also derived using principal components analysis (Alden et al., 1990). Factors were extracted based on observation of the scree plot and rotated orthogonally using the Procrustes procedure in Stata version 10 for Windows. Orthogonal rotations were used in order to match the underlying circumplex structure closely. Hypothesis (2) was investigated by computing the IIP-C structural summary (Gurtman and Balakrishnan, 1998) based on national gender norms (Horowitz et al., 2000). Angular scores were plotted in the circular space. To investigate hypothesis 
(3), correlations between pre- and post-treatment factor scores were evaluated. Because factor scores have means of zero, IIP-C parameters obtained from the structural summary were compared using paired-sample $t$-tests. Multilevel Modeling (MLM) was used to predict change in depressive symptom severity over the course of cognitive therapy as a function of pre-treatment interpersonal style and general distress (hypothesis 4). We applied intention-to-treat analysis, in which all available HRSD total scores were included (523 patients; 1-19 HRSD assessments). Time, interpersonal style, distress and the interactions among these factors were modeled as fixed effects and patients' intercepts as a random effect. Hypothesis (5) was investigated using multiple regression analysis. The WAI-C total score at Session 7 was entered as the dependent variable and the distress, agency and communion factors, as well as interactions among them, were entered as predictors. Non-significant interaction terms were removed from the model and the model was re-run.

\section{Results}

\section{Cross-sectional associations}

First we determined Pearson correlations among the study variables at the baseline assessment. The distress and agency factors related negatively to the WAI-C total score ( $r=-.13$ and $r=-.12$, respectively) whereas communion related positively to this variable $(r=.14)$. Moreover, Distress correlated negatively with symptom severity at baseline $(r=.23)$, whereas agency and communion were unrelated to baseline symptom severity ( $r=.04$ and $r=-.05$, respectively).

\section{IIP-C Circumplex Structure and Stability (Hypothesis 1)}

Separate principal components analysis of the pre- and post-treatment scales identified three orthogonal factors at each time point. The rotated factor loadings are summarized in Table 1. To visualize-and guide the interpretation of-the three factors and to demonstrate the circumplex structure of the IIP-C, factor loadings pre- and posttreatment were transformed into circular degree scores and plotted against the hypothetical circumplex structure (see Figure 1). Although the observed values did not converge perfectly with the expected circumplex structure, visual inspection of Figure 1 suggests that the circumplex space is adequately covered by the obtained factor loadings. To quantify the convergence between the theoretical circumplex structure and the obtained structure, correlations between the observed and expected angles were computed. The observed factor loadings both pre- and post-treatment correlated highly with the hypothesized circumplex structure (.99), strongly supporting our first hypothesis. 
Table 1. Rotated factor loadings of the Inventory of Interpersonal Problems - Circumplex Version

\begin{tabular}{|c|c|c|c|c|c|c|}
\hline \multirow[b]{2}{*}{ Scale } & \multicolumn{3}{|c|}{ Pre-treatment } & \multicolumn{3}{|c|}{ Post-treatment } \\
\hline & $\begin{array}{l}\text { Factor } 1 \\
\text { Distress }\end{array}$ & $\begin{array}{l}\text { Factor } 2 \\
\text { Agency }\end{array}$ & $\begin{array}{l}\text { Factor } 3 \\
\text { Communion }\end{array}$ & $\begin{array}{l}\text { Factor } 1 \\
\text { Distress }\end{array}$ & $\begin{array}{l}\text { Factor } 2 \\
\text { Agency }\end{array}$ & $\begin{array}{l}\text { Factor } 3 \\
\text { Communion }\end{array}$ \\
\hline Overly Nurturant & 0.75 & -0.20 & 0.63 & 0.64 & -0.10 & 0.56 \\
\hline Intrusive & 0.71 & 0.57 & 0.47 & 0.76 & 0.52 & 0.59 \\
\hline Domineering & 0.64 & 0.78 & -0.09 & 0.74 & 0.76 & -0.07 \\
\hline Vindictive & 0.75 & 0.47 & -0.48 & 0.68 & 0.44 & -0.52 \\
\hline Cold & 0.73 & 0.08 & -0.66 & 0.66 & 0.07 & -0.65 \\
\hline Socially Avoidant & 0.70 & -0.50 & -0.44 & 0.74 & -0.48 & -0.52 \\
\hline Nonassertive & 0.70 & -0.68 & 0.11 & 0.75 & -0.70 & 0.14 \\
\hline Exploitable & 0.72 & -0.52 & 0.46 & 0.69 & -0.50 & 0.47 \\
\hline
\end{tabular}

$N=510$ for pre-treatment factor scores and $N=356$ for post-treatment factor scores.

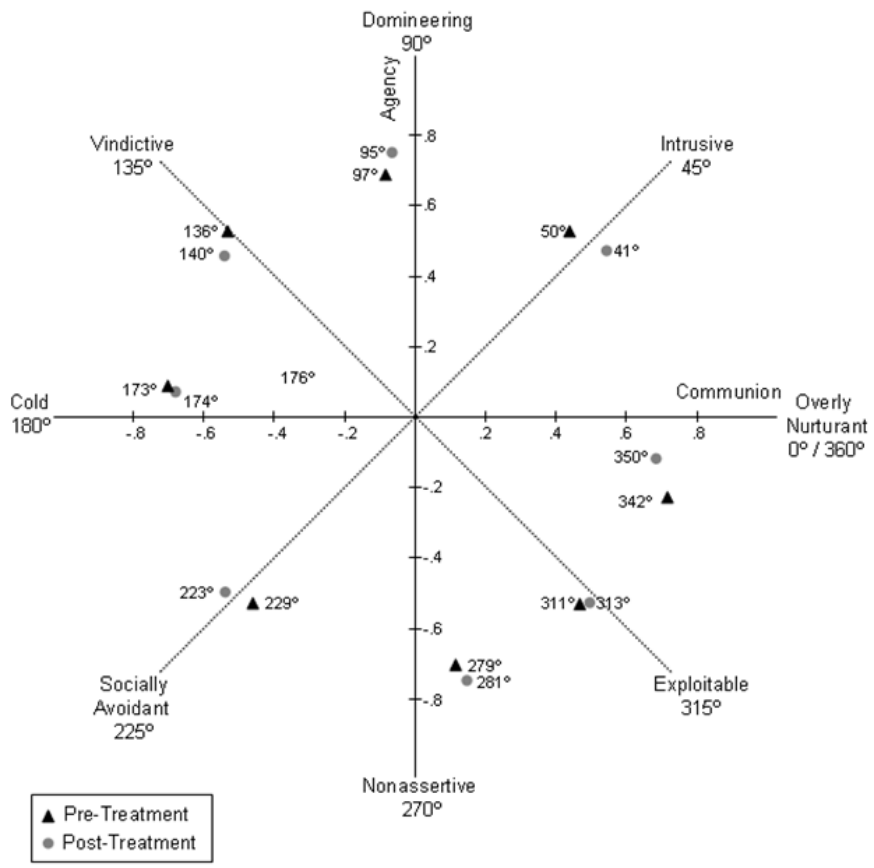

Figure 1. Angular placement of factor loadings for the Inventory of Interpersonal Problems - Circumplex Version pre- and post-treatment; Note. $N=510$; Numbers on the $\mathrm{x}$-axis and y-axis represent factor loadings for factor 1 (distress). 
Description of Interpersonal Problems Pre- and Post-Treatment (Hypothesis 2)

Descriptive and change statistics of the IIP-C octant scales using standardized z-scores are shown in Table 2. Socially avoidant, nonassertive and exploitable problems were somewhat more prominent both pre- and post-treatment, whereas intrusive, domineering and vindictive problems were less prominent both pre- and post-treatment. No octant scale means reached very high levels of distress (T-score $>70$ ) pre- or posttreatment. Patients in our sample improved statistically significantly on all octant scales, with medium effect sizes (Cohen, 1988), such that no octant scale means indicated clinically significant distress (T-score $>60$ ) post-treatment. To describe the sample's mean location in the circumplex space further, we computed the vector direction (angle) that reflected the most prominent form of interpersonal style. The results (see Figure 2) indicate that the predominant interpersonal style both pre- and post-treatment fell between social avoidance (225을 and non-assertiveness (270으.

Table 2. Descriptive and change statistics for the Inventory of Interpersonal Problems Circumplex Version pre- and post-treatment

\begin{tabular}{|c|c|c|c|c|c|c|c|}
\hline IIP-C Octant Scale & $\begin{array}{l}\text { Pre-treatment } \\
M(S D)\end{array}$ & $T$ score & $\begin{array}{l}\text { Post- treatm } \\
M(S D)\end{array}$ & $T$ score & $\begin{array}{l}\text { Paired } \\
t \text {-test }\end{array}$ & DF & $\operatorname{ES}(d)$ \\
\hline Overly Nurturant & $1.20(1.14)$ & 64 & $0.42(1.13)$ & 56 & 13.2 & 352 & 0.69 \\
\hline Intrusive & $0.67(1.16)$ & 57 & $0.11(1.06)$ & 51 & 11.1 & 352 & 0.58 \\
\hline Domineering & $0.73(1.25)$ & 59 & $0.13(1.13)$ & 50 & 10.4 & 352 & 0.50 \\
\hline Vindictive & $0.84(1.06)$ & 59 & $0.20(1.00)$ & 51 & 12.7 & 352 & 0.62 \\
\hline Cold & $1.14(1.06)$ & 61 & $0.42(1.05)$ & 54 & 13.7 & 353 & 0.68 \\
\hline Socially Avoidant & $1.62(1.24)$ & 67 & $0.77(1.25)$ & 58 & 14.1 & 352 & 0.68 \\
\hline Nonassertive & $1.62(1.20)$ & 66 & $0.89(1.17)$ & 59 & 12.7 & 354 & 0.62 \\
\hline Exploitable & $1.33(1.12)$ & 62 & $0.59(1.16)$ & 55 & 13.4 & 352 & 0.65 \\
\hline
\end{tabular}

$N=353-355$ (due to missing values on some subscales). All $t$-values are statistically significant at $p<0.01$ (corrected for multiple testing) and reflect decreased interpersonal problems post-treatment.

Effect sizes $(d s)$ were calculated by dividing the mean difference with the pooled standard deviation and may be interpreted as medium (Cohen, 1988). 


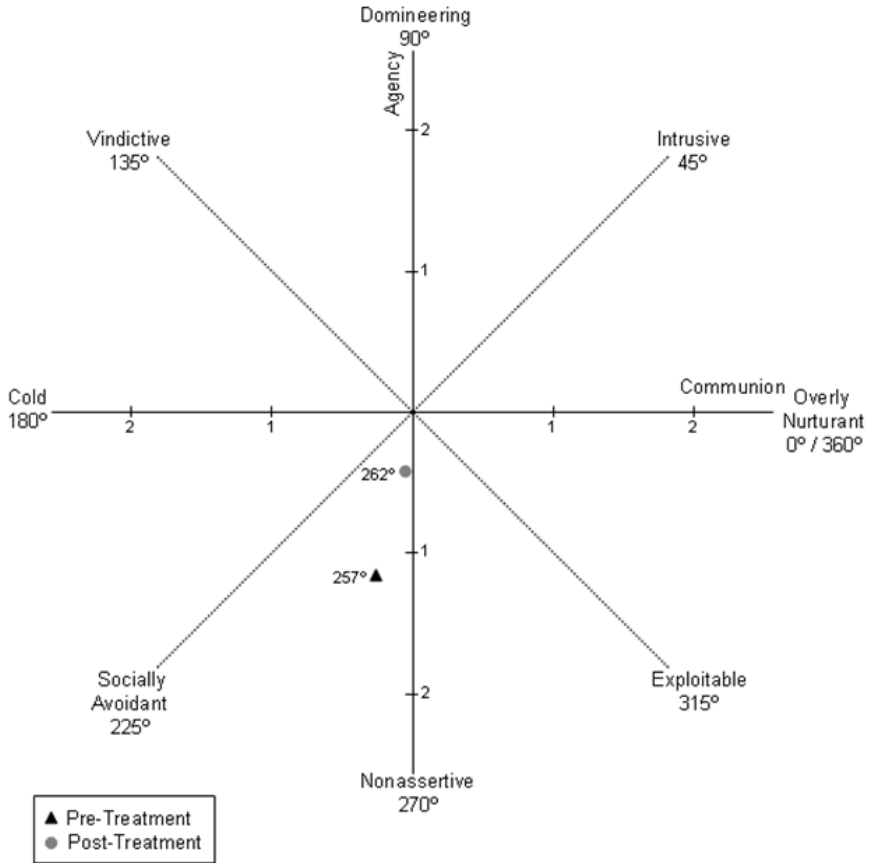

Figure 2. Predominant interpersonal style and mean general distress before and after acute-phase cognitive therapy;

Note. $N=356$; Numbers on the $\mathrm{x}$-axis and $\mathrm{y}$-axis represent standardized general distress scores, with scores above 1 representing clinical significant distress.

Stability and Change in General Distress and Interpersonal Style (Hypothesis 3)

Table 3 shows Pearson correlations between the pre- and post-treatment factor scores. Correlations between the pre- and post-treatment factors were moderate $(r=.54)$ for distress and somewhat stronger for agency and communion $(r=.68$ and $r=.74$, respectively). Moreover, both of the latter were statistically significantly stronger than the former, $z=-2.97, p<.01$; and $z=4.47, p<.01$, respectively. To investigate interpersonal changes further, mean changes of distress, agency and communion from pre-treatment to post-treatment were tested using closely related IIP-C parameters computed earlier. The general distress factor was approximated as the mean z-score of the IIP-C octant scales. The agency and communion factors were approximated by the normative agency and communion scale scores (Horowitz et al., 2000). The general distress, agency and communion factors converged strongly with these approximations both pre-treatment $(r \mathrm{~s}=1.00, .88, .85$, respectively) and post-treatment $(r \mathrm{~s}=$ $1.00, .82, .82$, respectively). 
Table 3. Correlations of the IIP-C factor scores pre- and post-treatment

\begin{tabular}{llll}
\hline & \multicolumn{2}{l}{ Post-treatment } \\
\cline { 2 - 4 } Pre-treatment & Distress & Agency & Communion \\
\hline Distress & $.54^{* *}$ & -.05 & .00 \\
Agency & .06 & $.68^{* *}$ & $.11^{*}$ \\
Communion & .02 & -.09 & $.74^{* *}$ \\
\hline
\end{tabular}

* Correlation is significant at $p<.05$ (two-tailed); ${ }^{* *}$ Correlations are significant at $p<.01$ (two-tailed); $N=$ 352 .

Table 4 summarizes the results of the paired samples $t$-tests between the pre- and posttreatment IIP-C parameters. General distress decreased significantly from pre-treatment $(M=1.15, S D=.75)$ to post-treatment $(M=.45, S D=.81 ; t(354)=17.60, p<.01, d=.90)$, whereas the communion dimension remained stable $(t(351)=-0.76 p>.05, d=.03)$. Scores on the agency dimension also increased significantly from pre-treatment $(M=$ $.47, S D=.79)$ to post-treatment $(M=-.37, S D=.67 ; t(352)=-3.28, p<.01)$, but the magnitude of this effect was small $(d=.15)$.

Table 4. Mean differences of IIP-C parameters distress, agency and communion as computed from the structural summary pre and post-treatment

\begin{tabular}{llllll}
\hline IIP-C Parameter & $\begin{array}{l}\text { Pre- treatment } \\
\text { Mean }(S D)\end{array}$ & $\begin{array}{l}\text { Post- treatment } \\
\text { Mean }(S D)\end{array}$ & Paired t-test & DF & Cohen's $d$ \\
\hline Distress & $1.15(.75)$ & $0.45(.81)$ & $17.60^{*}$ & 354 & .90 \\
Agency & $-.47(.79)$ & $-.37(.67)$ & $-3.28^{*}$ & 352 & .14 \\
Communion & $-.07(.65)$ & $-.05(.61)$ & -.76 & 351 & .03 \\
\hline
\end{tabular}

$*$ Mean difference is significant at $p<.01 ; N=352-355$.

Relations of Interpersonal Style and General Distress with Change in Depressive Symptoms (Hypothesis 4)

We used MLM to predict change in depressive symptoms over the course of cognitive therapy as a function of time, pre-treatment interpersonal style and general distress (Table 5). There were significant main effects for time, $F(18,6037.76)=461.12, p<.01$ and distress, $F(1,521.12)=24.82, p<.01$. To determine the relative importance of the effect of distress on symptom levels, we calculated standardized beta weights by standardizing all continuous variables before entering them into the model. The relation between general distress and symptom levels was relatively small $(\beta=.10)$. There was a significant interaction between distress and time, $F(18,6039.16)=2.06, p<.01$ and a marginally significant interaction between agency and time, $F(18,6038.06)=1.56, p$ $=.06$. To interpret these interaction terms, we plotted HRSD total scores over the course of cognitive therapy separately for the low- and high-distress groups and for the low- 
and high-agency groups (Figure 3), defined as one $S D$ below and above the respective IIP-C factor-score mean. Patients with higher baseline distress scores had significantly higher mean symptom scores over the course of treatment. Patients with high baseline agency had lower symptom scores in the middle of cognitive therapy and slightly lower symptom scores at the end of cognitive therapy.

Table 5. General distress, agency and communion as predictors of change in depressive symptom severity (HRSD) over the course of cognitive therapy

\begin{tabular}{lllll}
\hline Parameter & $\begin{array}{l}\text { Numerator } \\
\text { DF }\end{array}$ & $\begin{array}{l}\text { Denominator } \\
\text { DF }\end{array}$ & $F$ & $p$-value $(F)$ \\
\hline Intercept & 1 & 518.03 & 4003.54 & $<.01$ \\
Time & 18 & 6037.76 & 461.12 & $<.01$ \\
Distress & 1 & 521.12 & 24.82 & $<.01$ \\
Agency & 1 & 519.23 & 2.19 & $>.05$ \\
Communion & 1 & 513.81 & 0.30 & $>.05$ \\
Distress*Time & 18 & 6039.16 & 2.06 & $<.01$ \\
Agency*Time & 18 & 6038.06 & 1.56 & $>.05$ \\
Communion*Time & 18 & 6036.08 & 0.58 & $>.05$ \\
\hline
\end{tabular}

\section{Relations of Interpersonal Style and General Distress with Therapeutic Alliance} (Hypothesis 5)

The results of the multiple regression analysis are summarized in Table 6. High general distress and high agency were negatively related to WAI-C total scores $(\beta=-0.13, t(380)$ $=-2.64, p<0.01$ and $\beta=-0.12, t(380)=-2.46, p<0.05$, respectively), whereas high communion was positively related to WAI-C total scores $(\beta=.15, t(380)=2.91, p<0.01)$. This pattern of effects was found for all WAI-C subscales except between the general distress factor and task subscale. Whereas all variables in the model predicted therapeutic alliance in the expected direction, the total variance in WAI-C scores explained by these three factors was small $\left(R^{2}=.053\right)$. To determine whether the relations between interpersonal styles and distress with the therapeutic alliance remain once depressive symptom severity is controlled for, we added the HRSD total score at baseline to the regression model. All predictors remained significant and adding the HRSD baseline score did not improve the overall model $\left(\Delta R^{2}=0.0\right)$. Moreover, HRSD at baseline was unrelated to the WAI-C total score $(\beta=-0.03, t(380)=-0.54, p=.59)$. 
Estimated HRSD Scores by Baseline IIP Distress

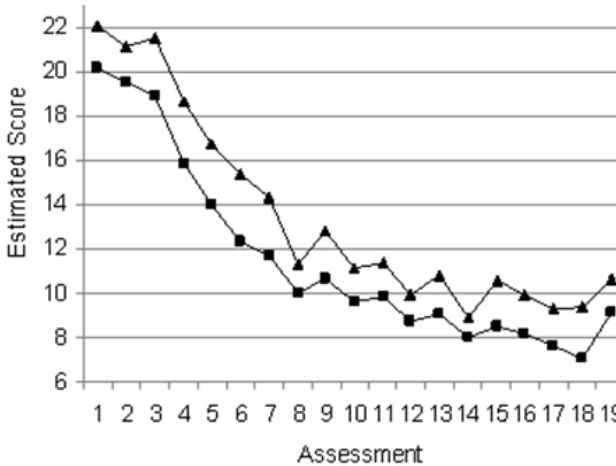

$\rightarrow$ Low Distress

$\rightarrow$ High Distress

Assessment

Estimated HRSD Scores by Baseline IIP Agency

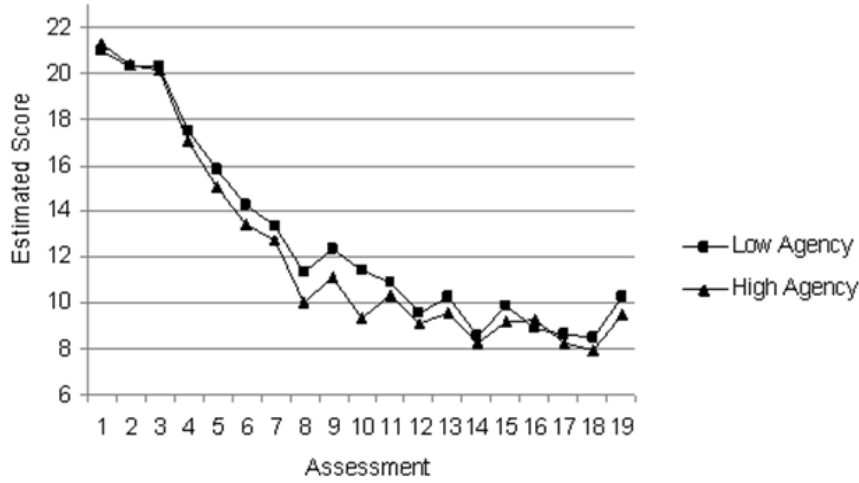

Figure 3. Change in depressive symptom severity over the course of CT predicted by IIP general distress and IIP agency. Note. Upper graph shows significant interaction between time moment and distress for the low and high distress group. Lower graph shows marginally significant interaction between time and agency for the low and high agency groups.

Table 6. General distress, agency, and communion as predictors of mid-treatment therapeutic alliance

\begin{tabular}{lllll}
\hline Factor & Total WAI-C & Task & Bond & Goal \\
$\beta(S E)$ & $\beta(S E)$ & $\beta(S E)$ & $\beta(S E)$ \\
\hline General Distress & $-0.13^{* *}(0.03)$ & $-0.09(0.04)$ & $-0.14^{* *}(0.04)$ & $-0.13^{*}(0.04)$ \\
Agency & $-0.12^{*}(0.03)$ & $-0.12^{*}(0.04)$ & $-0.12^{*}(0.04)$ & $-0.10^{*}(0.04)$ \\
Communion & $0.15^{* *}(0.03)$ & $0.14^{* *}(0.04)$ & $0.14^{* *}(0.04)$ & $0.12^{*}(0.04)$ \\
\hline
\end{tabular}

${ }^{*} p<0.05 ;{ }^{* *} p<0.01 ; N=384$. 


\section{Discussion}

We investigated the structure and stability of the IIP-C (Alden et al., 1990) over the course of a 16-to-20 session protocol of cognitive therapy in a large group of adult outpatients with recurrent MDD. Consistent with earlier studies, applying principal components analysis to IIP-C subscales (Holtforth et al., 2006; Horowitz et al., 1988; Vittengl et al., 2003), we found the hypothesized factor structure, consisting of general distress, agency and communion factors both before and after cognitive therapy. Moreover, when the agency and communion factor loadings were arranged in a circular space, they closely matched the corresponding circumplex octant scales' theoretical angular placements. This finding further supports the robustness of the IIP-C as a measure of interpersonal functioning.

In terms of angular placement in the circumplex, we found that the sample's predominant interpersonal style fell between social avoidance and non-assertiveness both before and after cognitive therapy. This is consistent with previous research investigating interpersonal profiles in patients with depression (Barrett and Barber, 2007). Moreover, although patients experienced significant distress in most areas of interpersonal functioning, on average, none of the eight interpersonal areas reached very high levels of interpersonal distress (i.e., means $>2 S D$ above the normative sample) either before or after cognitive therapy. However, $73 \%$ of individual patients reported very high levels of distress in at least one octant scale. Thus, patients with depression in this sample presented with a wide range of interpersonal problems with high levels of distress.

Following the description of interpersonal profiles, we investigated which aspects of interpersonal problems changed over the course of cognitive therapy. Consistent with previous research reporting improvement on most (Crits-Christoph et al., 2005) or all (Holtforth et al., 2006; Huber et al., 2007; Vittengl et al., 2003) IIP-C subscales, mean scores on all IIP-C subscales were reduced to non-clinical levels following therapy. Moreover, cross-correlations between pre- and post-treatment factor scores and meanscore comparisons of the corresponding IIP-C parameters revealed that this improvement was due largely to reduced distress, whereas interpersonal style remained largely stable, as hypothesized. Contrary to our expectations, agency also decreased at post-treatment, although the effect size was small and not likely to be clinically meaningful. As such, our findings are largely consistent with previous findings (CritsChristoph et al., 2005; Vittengl et al., 2003), suggesting that the IIP-C captures both statelike (general distress) and trait-like (interpersonal style) constructs.

Our findings differ from those of Holtforth et al. (2006) who reported change on all IIP-C dimensions after psychosocial treatment for depression. There are at least two possible explanations for the divergent findings in our study. First, patients in the Holtforth et al. (2006) study received a eclectic psychotherapy, with therapists free to draw upon process-experiential and interpersonal interventions in addition to cognitive-behavioral strategies. One prior study of 66 patients with MDD found that 
process-experiential therapy may be more effective in reducing interpersonal problems than cognitive therapy (Watson et al., 2003), but this hypothesis requires further testing. Perhaps more importantly, patients in the Holtforth et al. study received an average of 29 sessions (range $=5-127$ ) of eclectic psychotherapy, whereas out-patients in this study received a maximum of 20 cognitive therapy sessions. It has been shown previously that the number of therapy sessions is related, in a dose-response fashion, to improvement in interpersonal problems in patients with depression (Barkham et al., 2002).

The present study also addressed the predictive validity of the IIP-C for the therapeutic process and outcome. We tested interpersonal style and distress factor scores as predictors of change in depressive symptom severity over the course of cognitive therapy and hypothesized that the distress and agency factors would be related negatively to improvement of depressive symptoms, whereas the communion factor would be related positively to symptom improvement. In general, our findings that high levels of distress related negatively to symptom improvement whereas high agency levels related positively to symptom improvement are contrary to what we hypothesized based on theoretical grounds (Tracey, 1993) and prior research (Alden and Capreol, 1993; Gurtman, 1996; Muran et al., 1994). One reason for these divergent findings may be that previous studies categorized patients into quadrants of interpersonal problems based on their angular displacement score (Gurtman, 1996). Classification of patients into quadrants may oversimplify the diversity of the interpersonal problems experienced by patients with recurrent depression. Moreover, such a classification does not control for the general distress factor that should be detached from interpersonal style before investigating effects of interpersonal style on outcome. More valid results may be obtained by using dimensional scores, such as factor scores, that differentiate between interpersonal style and general distress.

Finally, we found support for our hypothesis that high scores on the communion factor at baseline would predict high alliance, whereas high scores on the agency and general distress factors would predict poor alliance. This finding is consistent with the idea that a friendly-submissive interpersonal style is complementary to the more active and directive style of cognitive therapists (Kiesler, 1983; Tracey, 1993). Although interpersonal style and distress at baseline consistently predicted therapeutic alliance in the expected direction, these effects were very small. In this context, it should be noted that the mean patients' alliance rating was very high, with very little variability $(M=6.1$, $S D=0.67$ ), probably reflecting a ceiling effect. Accordingly, interpersonal style and distress may explain a greater amount of total variance if there were more variation in the therapist-patient alliance.

\section{Limitations}

The study results should be interpreted in light of several limitations. First, given the study's inclusion/exclusion criteria, all patients had recurrent MDD. It is therefore uncertain whether or not these findings would generalize to the broader population of 
patients with other psychiatric disorders. Second, therapists' interpersonal style was not assessed. Because patients with an interpersonal style that is complementary to their therapists' may develop the best alliance, therapists' interpersonal style also should be accounted for rather than assuming that all therapists are friendly-dominant. Finally, the results of all analyses were based on a self-report measure of interpersonal problems. It has been shown that self-reported interpersonal problems on the IIP-C do not necessarily converge with observer-rated interpersonal problems (Leising et al., 2007).

\section{Implications}

Despite these limitations, the present study has important implications for clinical care and future research. First, the finding that the IIP-C showed a stable circumplex structure before and after cognitive therapy provides further evidence that the IIP-C can be used as a comprehensive measure of interpersonal constructs before and after treatment. Second, future research that uses the IIP-C should differentiate between interpersonal style and general distress, rather than treat interpersonal problems as a unidimensional construct. In the present study, interpersonal distress, but not interpersonal style, decreased during cognitive therapy. According to the cognitive theory of depression, dysfunctional thoughts are at the core of depression. Dysfunctional thoughts often contain interpersonal themes, such as the belief that one is inferior or will be rejected in interpersonal situations. Cognitive theory assumes that broad behavioral change across domains is possible, including reduction in interpersonal problem levels during cognitive therapy, as shown in the current analyses. At the same time, cognitive theory does not assume that the person's basic personality will change during short-term therapy. Therefore, our finding that interpersonal style remained stable during cognitive therapy for depression (i.e., although patients became less distressed about their interpersonal relations, their basic level of agency and communion did not change) is largely in line with what might be expected based on the cognitive theory of depression. Future research should investigate the stability of interpersonal distress and interpersonal style in other forms of psychotherapy that explicitly target personality or interpersonal functioning. Third, in the present study we determined the predominant interpersonal style of patients with MDD and related interpersonal style and distress to therapy outcome and the quality of the therapeutic alliance. Recent research in patients with anxiety disorders suggests that interpersonal subtypes exist within diagnostic homogeneous groups, and that these subtypes relate differently to treatment outcome (Cain et al., 2010; Salzer et al., 2011). Based on these studies, a next step might be to attempt to identify interpersonal subtypes in MDD. However, recent data in the personality trait domain indicates that subtyping is strongly sample-based and therefore should only be attempted on population representative samples (Eaton et al., 2010). Thus, identifying possible subtypes is an issue for future research. Whereas the present study further supported the use of the IIP-C as a comprehensive circumplex measure of interpersonal functioning, more research is needed investigating the predictive validity of the interpersonal style and distress 
components of the IIP-C in patients with different types of psychopathology and in different treatment settings. 


\section{References}

Alden, L.E., Capreol, M.J., 1993. Avoidant personality disorder: Interpersonal problems as predictors of treatment response. Behavior Therapy 24, 357-376.

Alden, L.E., Wiggins, J.S., Pincus, A.L., 1990. Construction of Circumplex Scales for the Inventory of Interpersonal Problems. J. Pers. Assess. 55, 521-536.

Alexander, L.B., Luborsky, L., Greenberg, L.S., Pinsof, W.M., 1986. The Penn Helping Alliance Scales. The psychotherapeutic process: A research handbook. Guilford Press, New York, NY US, pp. 325-366.

American Psychiatric Association, 1994. Diagnostic and Statistical manual of mental disorders (4th ed.). American Psychiatric Press, Washington, D.C.

Bagby, R.M., Ryder, A.G., Schuller, D.R., Marshall, M.B., 2004. The Hamilton Depression Rating Scale: Has the Gold Standard Become a Lead Weight? Am. J. Psychiatry 161, 2163-2177.

Barkham, M., Rees, A., Stiles, W.B., Hardy, G.E., Shapiro, D.A., 2002. Dose-effect relations for psychotherapy of mild depression: A Quasi-experimental comparison of effects of 2, 8, and 16 sessions. Psychotherapy Research 12, 463-474.

Barrett, M.S., Barber, J.P., 2007. Interpersonal profiles in major depressive disorder. J. Clin. Psychol. 63, 247-266.

Beck, A.T., Rush, A.J., Shaw, B.F., Emery, G., 1979. Cognitive therapy of depression. Guilford, New York.

Borkovec, T.D., Newman, M.G., Pincus, A.L., Lytle, R., 2002. A component analysis of cognitive-behavioral therapy for generalized anxiety disorder and the role of interpersonal problems. J. Consult. Clin. Psychol. 70, 288-298.

Cain, N.M., Pincus, A.L., Grosse Holtforth, M., 2010. Interpersonal Subtypes in Social Phobia: Diagnostic and Treatment Implications. J. Pers. Assess. 92, 514-527.

Cohen, J., 1988. Statistical power analysis for the behavioral sciences. Erlbaum, Hillsdale, NJ.

Connolly Gibbons, M.B., Crits-Christoph, P., de la Cruz, C., Barber, J.P., Siqueland, L., Gladis, M., 2003. Pretreatment expectations, interpersonal functioning, and symptoms in the prediction of the therapeutic alliance across supportive-expressive psychotherapy and cognitive therapy. Psychotherapy Research 13, 59-76.

Constantino, M., Smith-Hansen, L., 2008. Patient interpersonal factors and the therapeutic alliance in two treatments for bulimia nervosa. Psychotherapy Research 18, 683-698.

Crits-Christoph, P., Gibbons, M.B.C., Narducci, J., Schamberger, M., Gallop, R., 2005. Interpersonal Problems and the Outcome of Interpersonally Oriented Psychodynamic Treatment of GAD. Psychotherapy: Theory, Research, Practice, Training 42, 211-224.

Eaton, N.R., Krueger, R.F., South, S.C., Simms, L.J., Clark, L.A., 2010. Contrasting prototypes and dimensions in the classification of personality pathology: evidence that dimensions, but not prototypes, are robust. Psychol. Med. 41, 1151-1163.

Filak, J., Abeles, N., Norquist, S., 1986. Clients' pretherapy interpersonal attitudes and psychotherapy outcome. Professional Psychology: Research and Practice 17, 217-222.

First, M.B., Spitzer, R.L., Gibbon, M., Williams, J.B., 1996. Structured Clinical Interview for DSM-IV Axis I Disorders-Patient Edition (SCID-I/P, Version 2.0). New York State Psychiatric Institute, Biometrics Research Department, New York.

Gaston, L., 1991. Reliability and Criterion-Related Validity of the California Psychotherapy Alliance ScalesPatient Version. Psychological Assessment 3, 68-74.

Gurtman, M.B., 1996. Interpersonal problems and the psychotherapy context: The construct validity of the Inventory of Interpersonal Problems. Psychological Assessment 8, 241-255.

Gurtman, M.B., Balakrishnan, J.D., 1998. Circular measurement redux: The analysis and interpretation of interpersonal circle profiles. Clinical Psychology Science and Practice 5.

Hamilton, M., 1960. A rating scale for depression. J. Neurol. Neurosurg. Psychiatry 23, 56-61.

Hatcher, R.L., Barends, A.W., 1996. Patients' View of the Alliance in Psychotherapy: Exploratory Factor Analysis of Three Alliance Measures. J. Consult. Clin. Psychol. 64, 1326-1336. 
Holtforth, M.G., Lutz, W., Grawe, K., 2006. Structure and change of the IIP-D pre- and postpsychotherapy: A replication using a Swiss clinical sample. European Journal of Psychological Assessment 22, 98-103.

Horowitz, L.M., Alden, L.E., Wiggins, J.S., Pincus, A.L., 2000. Manual for the Inventory of Interpersonal Problems. Harcourt Assessment The Psychological Corporation, Oxford, UK.

Horowitz, L.M., Rosenberg, S.E., Baer, B.A., Ureno, G., Villasenor, V.S., 1988. Inventory of interpersonal problems: Psychometric properties and clinical applications. J. Consult. Clin. Psychol. 56, 885-892.

Horowitz, L.M., Rosenberg, S.E., Bartholomew, K., 1993. Interpersonal problems, attachment styles, and outcome in brief dynamic psychotherapy. J. Consult. Clin. Psychol. 61, 549-560.

Horowitz, L.M., Wilson, K.R., Turan, B., Zolotsev, P., Constantino, M.J., Henderson, L., 2006. How Interpersonal Motives Clarify the Meaning of Interpersonal Behavior: A Revised Circumplex Model. Personality \& Social Psychology Review 10, 67-86.

Horvath, A.O., Greenberg, L.S., 1989. Development and validation of the Working Alliance Inventory. Journal of Counseling Psychology 36, 223-233.

Horvath, A.O., Symonds, B.D., 1991. Relation between working alliance and outcome in psychotherapy: A meta-analysis. Journal of Counseling Psychology 38, 139-149.

Huber, D., Henrich, G., Klug, G., 2007. The Inventory of Interpersonal Problems (IIP): Sensitivity to change. Psychotherapy Research 17, 474-481.

Jarrett, R.B., Thase, M.E., 2010. Comparative efficacy and durability of continuation phase cognitive therapy for preventing recurrent depression: Design of a double-blinded, fluoxetine- and pill placebo-controlled, randomized trial with 2-year follow-up. Contemporary Clinical Trials 31, 355377.

Kiesler, D.J., 1983. The 1982 Interpersonal Circle: A taxonomy for complementarity in human transactions. Psychol. Rev. 90, 185-214.

Kiesler, D.J., Watkins, L.M., 1989. Interpersonal complementarity and the therapeutic alliance: A study of relationship in psychotherapy. Psychotherapy: Theory, Research, Practice, Training 26, 183-194.

Leising, D., Rehbein, D., Sporberg, D., 2007. Validity of the Inventory of Interpersonal Problems (IIP-64) for predicting assertiveness in role-play situations. J. Pers. Assess. 89, 116-125.

Muran, J.C., Segal, Z.V., Samstag, L.W., Crawford, C.E., 1994. Patient pretreatment interpersonal problems and therapeutic alliance in short-term cognitive therapy. J. Consult. Clin. Psychol. 62, 185-190.

Paivio, S.C., Bahr, L.M., 1998. Interpersonal problems, working alliance, and outcome in short-term experiential therapy. Psychotherapy Research 8, 392-407.

Puschner, B., Bauer, S., Horowitz, L.M., Kordy, H., 2005. The Relationship Between Interpersonal Problems and the Helping Alliance. J. Clin. Psychol. 61, 415-429.

Ruiz, M.A., Pincus, A.L., Borkovec, T.D., Echemendia, R.J., Castonguay, L.G., Ragusea, S.A., 2004. Validity of the Inventory of Interpersonal Problems for Predicting Treatment Outcome: An Investigation With The Pennsylvania Practice Research Network. J. Pers. Assess. 83, 213-222.

Salzer, S., Pincus, A.L., Winkelbach, C., Leichsenring, F., Leibing, E., 2011. Interpersonal subtypes and change of interpersonal problems in the treatment of patients with generalized anxiety disorder: A pilot study. Psychotherapy 48, 304-310.

Schauenburg, H., Kuda, M., Sammet, I., Strack, M., 2000. The influence of interpersonal problems and symptom severity on the duration and outcome of short-term psychodynamic psychotherapy. Psychotherapy Research 10, 133-146.

Tracey, T.J., 1993. An interpersonal stage model of the therapeutic process. Journal of Counseling Psychology 40, 396-409.

Vittengl, J.R., Clark, L.A., Jarrett, R.B., 2003. Interpersonal problems, personality pathology, and social adjustment after cognitive therapy for depression. Psychological Assessment 15, 29-40.

Watson, J.C., Gordon, L.B., Stermac, L., Kalogerakos, F., Steckley, P., 2003. Comparing the effectiveness of process-experiential with cognitive-behavioral psychotherapy in the treatment of depression. J. Consult. Clin. Psychol. 71, 773-781.

Wiggins, J.S., 1982. Circumplex models of interpersonal behavior in clinical psychology. In: Kendall, P.C. and Butcher, J.N. (Eds.), Handbook of research methods in clinical psychology. Wiley, New York, pp. 183-221. 


\section{CHAPTER 7 \\ Effects of psychotherapy for depression on social functioning}

Published as:

Renner, F., Cuijpers, P., \& Huibers, M. J. H. (2014). The effect of psychotherapy for depression on improvements in social functioning: a meta-analysis. Psychological Medicine, 44 (14), 2913-2926. 


\begin{abstract}
Background: Patients with depression often report impairments in social functioning. From a patient perspective, improvements in social functioning might be an important outcome in psychotherapy for depression. Therefore, it is important to examine the effects of psychotherapy on social functioning in patients with depression.
\end{abstract}

Method: We conducted a meta-analysis on studies of psychotherapy for depression that reported results for social functioning at post-treatment. Only studies that compared psychotherapy to a control condition were included (Thirty-one studies with 2956 patients).

Results: The effect size of psychotherapy on social functioning was small to moderate, before ( $\mathrm{g}=0.46$; 95\% CI:0.32;0.60) and after adjusting for publication bias ( $\mathrm{g}=0.40 ; 95 \%$ CI:0.25;0.55). Univariate moderator analyses revealed that studies using care-as-usual as a control group versus other control groups, studies conducted in the United States versus other countries and studies that used clinician-rated instruments versus selfreport yielded higher effect sizes. Higher quality studies yielded lower effect sizes whereas the number of treatment sessions and the effect size on depressive symptoms were positively related to the effect size of social functioning. When controlling for these and additional characteristics simultaneously in multivariate metaregression, the effect size of depressive symptoms, treatment format and number of sessions were significant predictors. The effect size of social functioning remained marginally significant, indicating that improvements in social functioning are not fully explained by improvements in depressive symptoms.

Conclusions: Psychotherapy for depression results in small to moderate improvements in social functioning. These improvements are strongly associated with but not fully explained by improvements in depressive symptoms.

Keywords: Depression, Social functioning, Psychotherapy, Meta-analysis 


\section{Introduction}

Social functioning can be defined as the degree to which a person is able to fulfil various roles in different social environments such as at work, at home, in relationships or during leisure activities (Bosc, 2000). Patients with depression usually report deficits in social functioning in addition to depressive symptoms (Hirschfeld et al., 2000). These impairments are often long-lasting and are equal to or exceed those of chronic medical illnesses such as myocardial infarction and diabetes (Hays et al., 1995). Impairments in social functioning in depression strongly contribute to the increased burden of depression (Eaton et al., 2008, Greenberg et al., 2003) and predict depressive relapse and recurrences (e.g. Judd et al., 2000, Leon et al., 1999, Solomon et al., 2004, Vittengl et al., 2009). Impairments in social functioning in depression are also related to high economic costs. The impact of depression on work functioning, which is one important aspect of the broader concept of social functioning, often exceeds those of other chronic illnesses. In 2011 about 13.3 million work days were lost due to depression and anxiety disorders in the UK (Office of National Statistics, 2012). Findings like this stress the importance of including social functioning as an outcome measure in treatment studies of depression.

Treatment outcome studies in depression usually focus on the effects of treatment in improving depressive symptom severity rather than social functioning. For example, several meta-analyses have shown that different types of psychotherapy for depression are moderately effective in reducing depressive symptoms (e.g., Barth et al., 2013, Cuijpers et al., 2008a) but no meta-analysis to date has quantified the effects of psychotherapy on social functioning in depression. Definitions of response and remission in depression outcome studies are exclusively based on relative and absolute improvements in symptom severity. Although measures of symptom severity usually also include, to some extent, aspects of social functioning, improvements in social functioning are usually not explicitly included in the definition of response, remission or recovery from depression. The World Health Organization conceptualizes health as "...a state of complete physical, mental and social well-being and not merely the absence of disease or infirmity" (World Health Organization, 1948, p. 2). Moreover, from a patient perspective improvements in social functioning might be a preferred treatment outcome. For example, Zimmerman et al. (2006) found that among 535 outpatients with MDD a return to a normal level of functioning at work, home or school was rated among the three most important factors in determining whether a depression was in remission. Similarly, in a qualitative study on treatment goals of depressed outpatients it was found that patients with MDD commonly articulate improving one's social and family relationships as desired treatment goals (Battle et al., 2010). Mintz and colleagues (1992) compiled data from 10 published treatment studies (psychotherapy and antidepressants) and found that one aspect of social functioning, work impairment, improved with symptom remission, although improvements in work functioning were slower than symptomatic improvements. 
Given that no meta-analysis to date has estimated the effects of psychotherapy for depression on social functioning, it is not clear how large the effects of psychotherapy for depression on social functioning are, whether these effects are smaller, equal or exceed the effects of psychotherapy on symptom severity and whether these effects differ between different types of psychotherapy. The aim of this meta-analysis was to summarize the effects of psychotherapy for depression on improvements in social functioning. We hypothesized that psychotherapy for depression has positive effects on social functioning; that there are no differences between different types of psychotherapy for depression on improvements in social functioning; and that the effects of psychotherapy for depression on social functioning are positively correlated with the effects of psychotherapy for depression on depressive symptom severity.

\section{Methods}

\section{Study selection}

For study selection we used a database of 1476 papers on the psychological treatment for depression. A more detailed description of the methods that were used to build the database is available elsewhere (Cuijpers et al., 2008c). This database is continuously updated, available to other researchers (www.evidencebasedpsychotherapies.com) and has been used in a series of meta-analyses previously.

For the current meta-analyses we included studies comparing the effects of psychological interventions for depression with a control condition (waiting-list, careas-usual, or placebo). We further included only studies that classified depression according to a diagnostic interview or elevated depression levels on validated selfreport questionnaires. Studies in children and adolescents (age below 18) were excluded, as were studies that did not provide post-treatment means and standard deviations (or other statistics from which these could be computed) of depression severity or social functioning. Studies on continuation or maintenance treatments were also excluded as were studies on unguided self-help treatments. We defined instruments that assess social functioning as any instruments that have the goal to assess functioning in any of the following social domains: interpersonal contacts, partner relation, work or study, leisure or recreation activities. Studies specifically assessing marital or dyadic adjustment or studies assessing global functioning were not included. We included studies that assessed social functioning based on self-report, observer or clinician ratings.

\section{Quality assessment}

We used four criteria from the Cochrane risk of bias assessment tool (Higgins et al., 2011) to assess the quality of the included studies: random sequence generation (rated positive if the randomization scheme was generated correctly); allocation concealment (rated positive if allocation to conditions was conducted by an independent party); 
blinding of outcome assessment (rated positive if assessors were blind to treatment condition); handling of incomplete outcome data (rated positive if intention-to-treat analyses were conducted).

\section{Statistical analyses}

For the univariate analyses we used the program Comprehensive Meta-Analysis. We calculated the effect size (Hedges $g$ ) for each comparison between psychotherapy and a control group as an indicator of the difference between the two groups at post-test. To calculate the effect sizes the average score of the experimental group at post-test was subtracted from the average score of the control group at post-test and the result was divided by the pooled standard deviation. To correct for small sample bias we followed the procedures suggested by Hedges and Olkin (1985). We calculated separate effect sizes for social functioning and depression. Mean effect sizes were calculated using a random effects model in order to account for the heterogeneity among studies. The random effects model assumes that all included studies are estimating different (underlying) effect sizes. To test the homogeneity of effect sizes we calculated the $I^{2}$ and the $Q$-statistic (Higgins et al., 2003). Publication bias was assessed by visual inspection of the funnel plot and by the trim and fill procedure which provides an adjusted estimate of the overall effect size, after adjusting for publication bias (Duval and Tweedie, 2000). A random effects model was used to look for missing studies.

We used meta-regression analyses to test whether there was a relationship between the effect sizes of social functioning and the effect sizes of depression severity. For categorical study characteristics a series of subgroup or moderator analyses was conducted using a mixed effect model. Tests for significance between the different subgroups were conducted using a fixed effects model. For continuous variables, metaregression analyses were used. Multivariate analyses were conducted using Stata/SE 12.0 for Windows. In these analyses the weighted effect size in social functioning at post-treatment was the dependent variable and the effect size of depressive symptom severity at post-treatment along with potential confounding variables were entered as predictors.

\section{Results}

\section{Selection and inclusion of studies}

Three hundred fifty two Randomized Clinical Trials (RCTs) were included in the database. Of these studies 204 included a control group. Of these studies, and the corresponding duplicate studies, the methods section and results sections were checked to determine whether a measure of social functioning was included. Thirty-one studies met our inclusion criteria, reported social functioning at post-test and were included in the meta-analyses comparing the effects of psychotherapy (versus control group) on social functioning. 


\section{Description of included studies}

Characteristics of the included studies are provided in Table 1. The total number of participants in the 31 included studies was 2,956 (1,655 in the treatment condition and 1,301 in the control conditions). In 11 of the 31 studies participants were recruited through the community, 13 used clinical referral and seven relied on other recruitment strategies. Seventeen studies focused on adults in general, eight studies only included women (two only women with postpartum depression, one only infertile women, one only women seeking care following miscarriage, one only women with a history of childhood trauma), five studies specifically focused on older adults, and one study included adults with a general medical condition. Some studies compared more than one type of psychotherapy to a control group resulting in a total number of 39 comparisons between psychotherapy and a control group. In 18 of the 39 comparisons CBT (including three studies on coping with depression course) was compared to a control group; six comparisons were between IPT and a control group; four comparisons were between problem solving and a control group and the remaining 11 comparisons were between a control group and acceptance and commitment therapy, behavioural activation, dialectic behavioural therapy, guided self-help, integrative and instrumental reminiscence therapies, interpersonal counselling, psychodynamic therapy or supportive therapy. The control group consisted of care-as-usual (19 studies), wait-list-control (eight studies) or other control conditions (four studies; active socialization, meditation, pill placebo). Various instruments were used to assess social functioning: the social adjustment scale (self-report or clinician rated or modified version; Weissman et al., 1978) was used by 14 studies; the social functioning subscale of the Medical Outcomes Study Short-Form Health Survey (Ware and Sherbourne, 1992) was used by five studies; the inventory of interpersonal problems (Horowitz et al., 2000) was used by two studies; two studies used the Sheehan disability scales (Sheehan, 1983) to assess social functioning; the remaining studies used other instruments to assess social functioning.

\section{Quality of included studies}

Sixteen studies reported to have used an adequate sequence generation (method to generate the random allocation sequence); in 18 studies random allocation to conditions was conducted by an independent researcher; in 24 studies assessors were blind to treatment condition; intention-to-treat analyses was conducted in 23 of the included studies. Eight studies met all four quality criteria. 


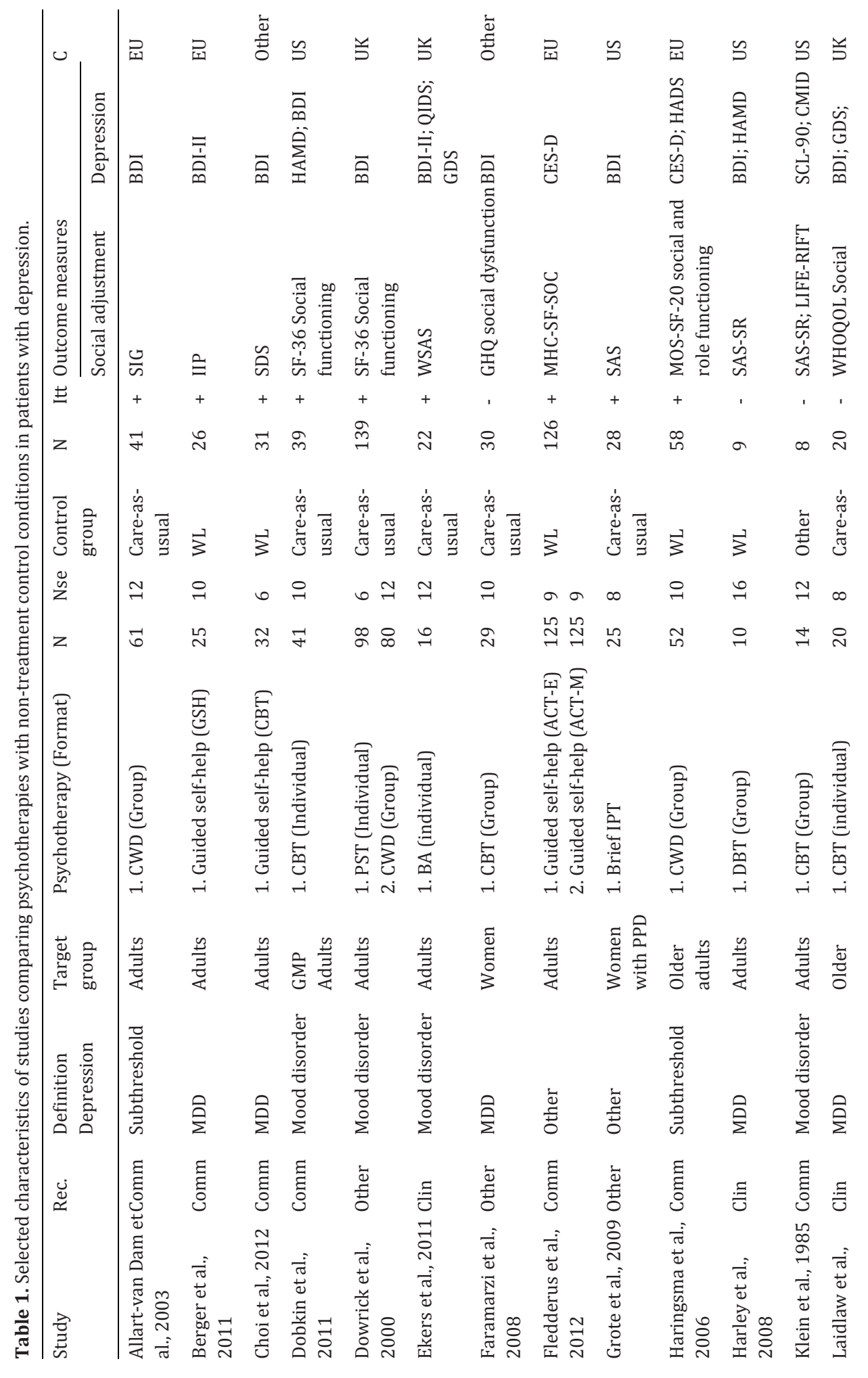




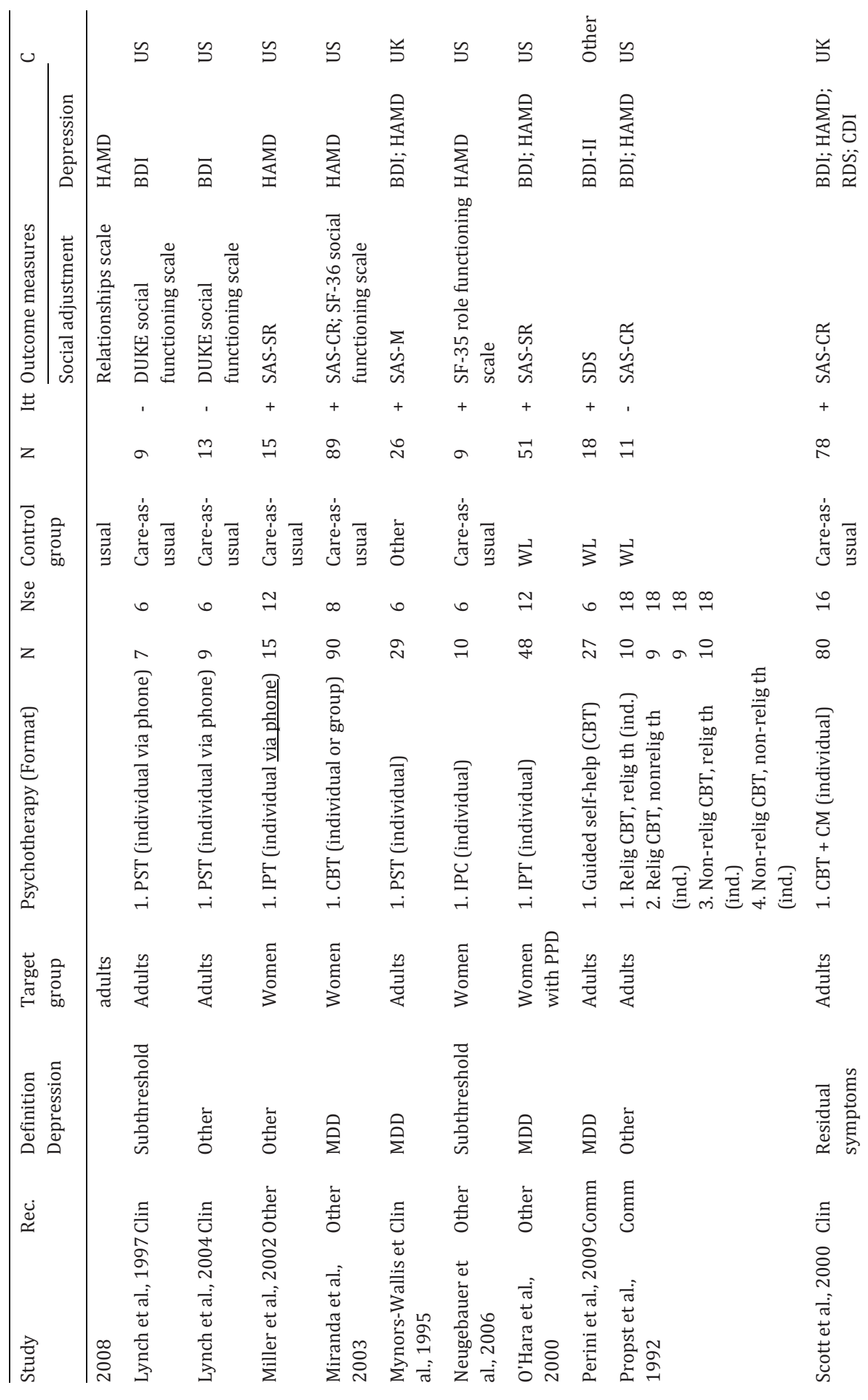




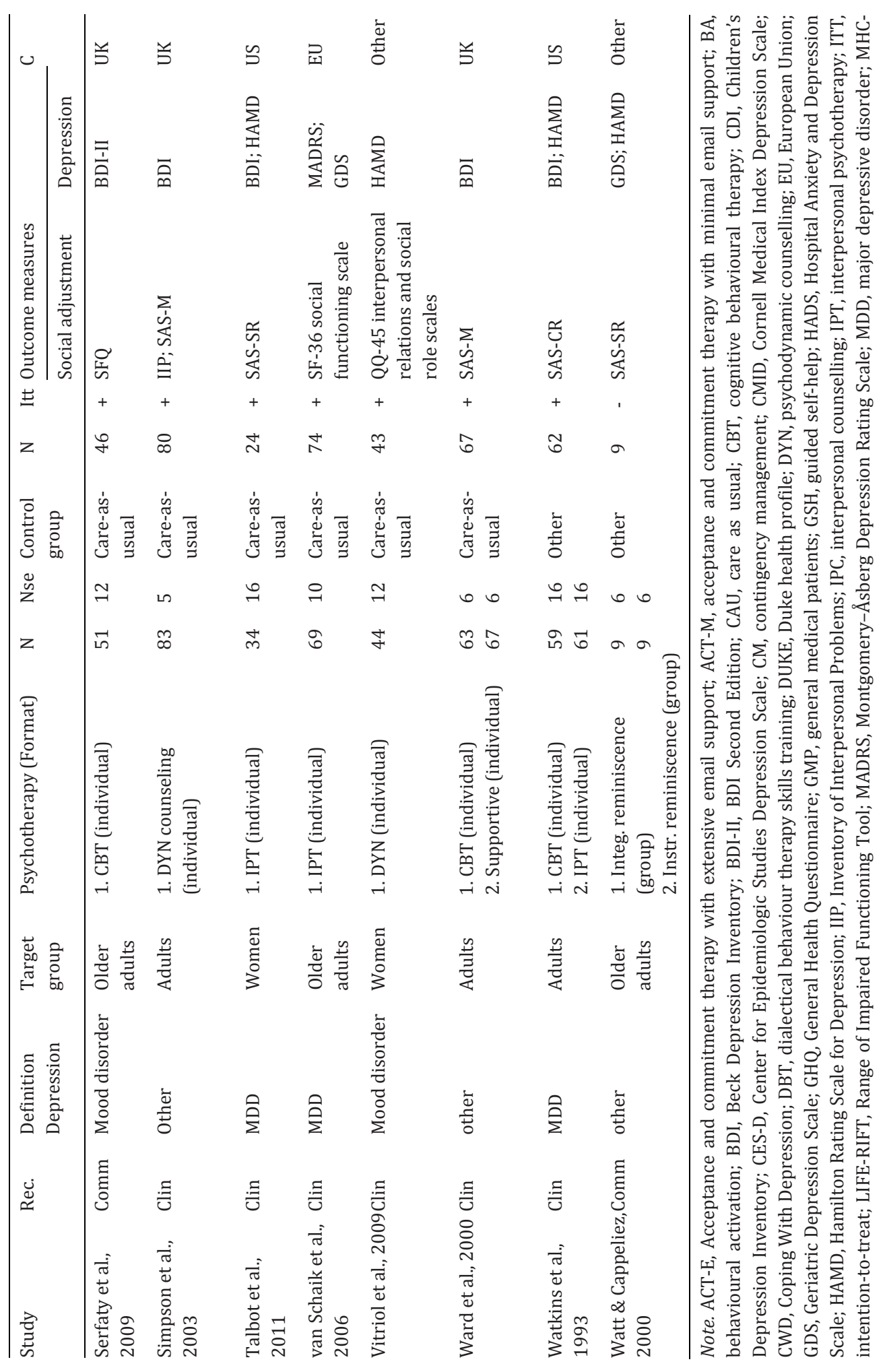




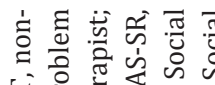

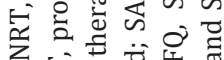

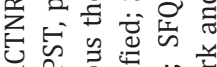

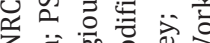
$z$. बे एक जि के 워뇨

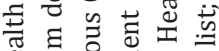

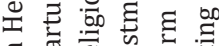

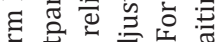

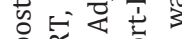
包 恶 $\frac{d}{3}$ के घี

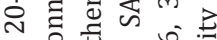

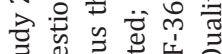
क必的。 \% है छั 氙苛引元 ㄴำ

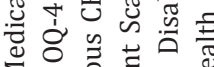
$\sum \ddot{0}$ षี 긍 푱 등 i 的安的家

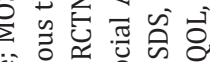
แ. 언 जि ○ 节产过 元

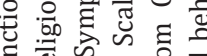
छ क

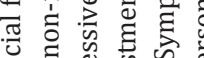

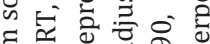
हा 원 क्षे के

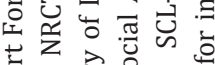

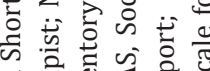

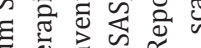
寻宁过

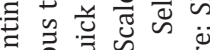
త 퓰

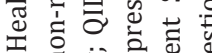

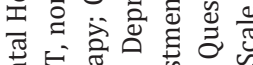
वี ن 을 요

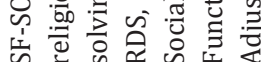




\section{Meta-Analyses}

\section{Effects of psychotherapy on social functioning}

Between study heterogeneity was large $\left(Q=129.22 ; p<.001 ; I^{2}=70.59\right)$ and therefore random effect models were used in subsequent analyses. Figure 1 provides an overview of the effect sizes of social functioning per study. The mean effect of psychotherapy on social functioning was small to moderate (Hedges' $g=0.46 ; 95 \% \mathrm{CI}: 0.32 ; 0.60$ ) and significantly different from zero $(z=6.42, p<.001)$.

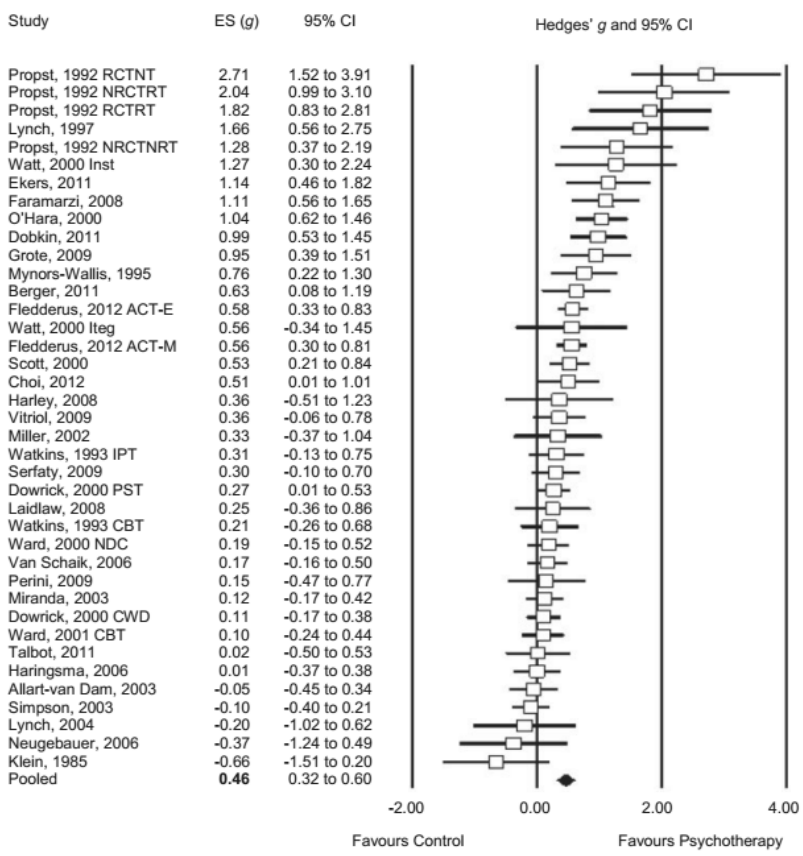

Figure 1. Standardized effect sizes (Hedges' g) of the effects of psychotherapy for depression on social functioning compared to control conditions. ACT-E, Acceptance and commitment therapy with extensive email support; ACT-M, acceptance and commitment therapy with minimal email support; CBT, cognitive behavioural therapy; CI, confidence interval; CWD, Coping With Depression course; Inst, instrumental reminiscence; Integ, integrative reminiscence therapy; IPT, interpersonal psychotherapy; NDC, nondirective counselling; NRCTNRT, non-religious CBT, non-religious therapist; NRCTRT, non-religious CBT, religious therapist; PST, problem solving therapy; RCTNT, religious CBT, non-religious therapist; RCTRT, religious CBT, religious therapist

The funnel plot and Duvall and Tweedie's Trim and Fill procedure suggested the presence of possible publication bias (Figure 2). After adjusting for publication bias using the Trim and Fill procedure, the overall effect size for the random effects model was $g=0.40$ (95\% CI:0.25;0.55). 


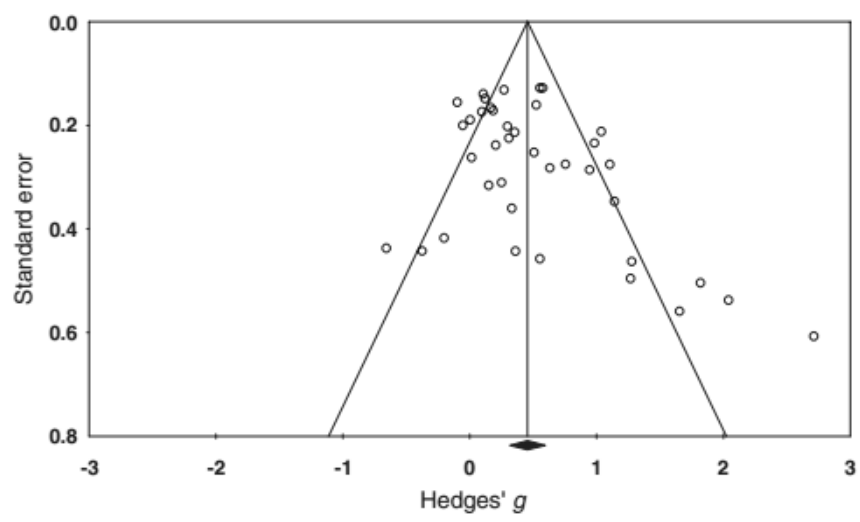

Figure 2. Funnel plot of standard error by Hedges's g.

We also computed the effect size of psychotherapy on depressive symptom severity in the included studies. The effect size of psychotherapy on depressive symptom severity was moderate (Hedges' $g=0.58 ; 95 \%$ CI:0.44;0.72) and significantly different from zero $(z=8.06, p<.001)$. After adjusting for publication bias using the Trim and Fill procedure, the overall effect size for the random effects model dropped to $g=0.43 ; 95 \%$ CI:0.28;0.58).

\section{Sensitivity analyses}

In six studies more than one type of psychotherapy was compared with the same control group and therefore the effect sizes from these comparisons were not independent. We conducted two sensitivity meta-analyses by first including only the largest effect size per study and then only including the smallest effect size per study. When only including the largest effect sizes, the overall effect size was (Hedges' $g=0.43 ; 95 \% \mathrm{CI}: 0.27 ; 0.58$ ) and significantly different from zero $(z=5.43, p<.001)$. These estimates did not change after adjusting for publication bias. When only including the smallest effect sizes, the overall effect size was (Hedges' $g=0.38 ; 95 \% \mathrm{CI}: 0.24 ; 0.53$ ) and significantly different from zero $(z=5.16, p<.001)$. These estimates did not change after adjusting for publication bias.

One study was based on data of patients with residual depressive symptoms (Scott et al., 2000). In a sensitivity analysis, excluding this study, the effect size remained the same ( $\mathrm{g}=0.46 ; 95 \% \mathrm{CI}: 0.31 ; 0.60$ ). To test if one single individual study had a strong impact on the overall effect size, we conducted a number of additional sensitivity analyses in which the effect size was computed after excluding one study. In these analyses the effect size estimates ranged from 0.43 to 0.48 , indicating that no individual study had a strong impact on the overall effect size.

\section{Subgroup analyses}

The results of the univariate subgroup analyses for studies comparing psychotherapies to a control group are shown in Table 2. Studies comparing psychotherapy to care-asusual resulted in smaller effect sizes ( $g=0.33,95 \% \mathrm{CI}: 0.16$; 0.50$)$, compared to studies 
comparing psychotherapy to other control groups ( $g=0.63,95 \% \mathrm{CI}: 0.42 ; 0.84, p<.001)$. Studies that used an individual treatment format resulted in larger effect sizes $(g=0.50$, $95 \% \mathrm{CI}: 0.33$; 0.67$)$ compared to studies that used a group format $(g=0.28,95 \% \mathrm{CI}:-0.05$; $0.60, p<.01)$. Moreover, studies that were conducted in the US resulted in larger effect sizes ( $g=0.55,95 \% \mathrm{CI}: 0.34 ; 0.75)$, compared to studies that were conducted outside the US ( $g=0.38,95 \% \mathrm{CI}: 0.19 ; 0.56, p<.01)$. Finally, studies that used clinical rating scales to assess social functioning reported larger effect sizes $(g=0.91,95 \%$ CI:0.54; 1.28) compared to studies that relied on self-reported social functioning $(g=0.39,95 \%$ CI:0.24; $0.55, p<.01)$.

We also tested whether studies that compared IPT or CBT respectively, to a control group resulted in differential effect sizes compared to studies comparing other types of treatment to a control group. No differences in effect size estimates emerged for studies comparing IPT to a control group versus studies comparing other types of treatment to a control group ( $p=.42$ ) or studies comparing CBT to a control group versus studies comparing other types of treatment to a control group ( $p=.57)$. To further investigate whether one broader cluster of psychotherapies resulted in differential effect sizes, we pooled the different types of psychotherapies into 2 broad clusters: The cognitivebehavioural cluster (acceptance and commitment therapy, behavioural activation, cognitive behavioural therapy, coping with depression course, dialect behavioural therapy, problem solving therapy) and a psychodynamic-interpersonal cluster (interpersonal psychotherapy, interpersonal counselling, psychodynamic counselling). Studies comparing treatments from the cognitive-behavioural cluster to control groups resulted in effect sizes $(g=0.50,95 \% \mathrm{CI}: 0.32 ; 0.68)$ comparable to those in studies comparing treatments from the psychodynamic-interpersonal cluster to a control group ( $g=0.32$, 95\% CI:0.31; $0.60, p=.14$ ). The other study characteristics were unrelated to the effect size of social functioning.

\section{Meta-Regression-Analysis}

We conducted a series of meta-regression analyses to test whether selected study characteristic were related to the effectsize of psychotherapy at post-treatment. There was a significant association between the effect size of social functioning and the effect size of depression (slope: $0.71 ; 95 \%$ CI:0.55;0.88; $p<.001$ ) suggesting that with each increase in the effect size of depressive symptom severity by one, the effect size of social functioning increased by 0.71 (see Figure 3). The number of treatment sessions was also statistically significantly related to the effect size (slope: $0.03 ; 95 \% \mathrm{CI}: 0.01 ; 0.05 ; p<.01$ ), indicating that studies with more treatment sessions resulted in higher effect sizes. The slope of 0.03 indicates that for each increase in 5 sessions, an increase in the effect size for social functioning of 0.15 was observed. 
Table 2. Meta-analyses of studies comparing psychotherapy vs. control groups: Subgroup analyses testing associations between effect sizes and study characteristics.

\begin{tabular}{|c|c|c|c|c|c|c|c|}
\hline Subgroup Analysis & Ncomp & ES (g) & $95 \% \mathrm{CI}$ & $\mathrm{Z}$ & $\mathrm{Q}$ & $\mathrm{I}^{2}$ & $\mathrm{p}$ \\
\hline \multicolumn{8}{|l|}{ Control Group } \\
\hline Care as Usual & 21 & 0.33 & $0.16 ; 0.50$ & 3.71 & 55.55 & 64.00 & \multirow[t]{2}{*}{$<.001$} \\
\hline Other & 18 & 0.63 & $0.42 ; 0.84$ & 5.90 & 58.07 & 70.73 & \\
\hline \multicolumn{8}{|l|}{ Format } \\
\hline Individual & 26 & 0.50 & $0.33 ; 0.67$ & 5.65 & 95.17 & 73.73 & \multirow[t]{2}{*}{$<.01$} \\
\hline Group & 8 & 0.28 & $-0.05 ; 0.60$ & 1.69 & 23.00 & 69.57 & \\
\hline \multicolumn{8}{|l|}{ Target Group } \\
\hline Adults & 33 & 0.48 & $0.33 ; 0.63$ & 6.22 & 119.96 & 73.32 & \multirow[t]{2}{*}{.10} \\
\hline Older Adults & 6 & 0.32 & $-0.05 ; 0.68$ & 1.71 & 6.56 & 23.75 & \\
\hline \multicolumn{8}{|c|}{ Definition Depression } \\
\hline MDD & 19 & 0.39 & $0.20 ; 0.59$ & 3.96 & 46.61 & 61.38 & \multirow[t]{2}{*}{.51} \\
\hline Other & 20 & 0.53 & $0.32 ; 0.74$ & 5.04 & 82.10 & 76.86 & \\
\hline \multicolumn{8}{|l|}{ Country } \\
\hline US & 20 & 0.55 & $0.34 ; 0.75$ & 5.27 & 73.87 & 74.28 & \multirow[t]{2}{*}{$<.01$} \\
\hline Other & 19 & 0.38 & $0.19 ; 0.56$ & 4.00 & 45.95 & 60.83 & \\
\hline \multicolumn{8}{|l|}{ Instrument used } \\
\hline Self-Report & 31 & 0.39 & $0.24 ; 0.55$ & 4.97 & 88.14 & 65.97 & \multirow[t]{2}{*}{$<.01$} \\
\hline Clinician Rated & 7 & 0.91 & $0.54 ; 1.28$ & 4.83 & 31.73 & 81.09 & \\
\hline \multicolumn{8}{|l|}{ Gender } \\
\hline Females only & 8 & 0.48 & $0.17 ; 0.79$ & 3.05 & 27.63 & 74.66 & \multirow[t]{2}{*}{.32} \\
\hline Mixed group & 31 & 0.45 & $0.29 ; 0.61$ & 5.59 & 100.59 & 70.18 & \\
\hline \multicolumn{8}{|l|}{ IPT vs. other } \\
\hline IPT & 6 & 0.47 & $0.12 ; 0.81$ & 2.65 & 16.78 & 70.20 & \multirow[t]{2}{*}{.42} \\
\hline Other & 33 & 0.46 & $0.30 ; 0.61$ & 5.78 & 111.79 & 71.37 & \\
\hline \multicolumn{8}{|l|}{ CBT vs. other } \\
\hline CBT & 18 & 0.51 & $0.30 ; 0.72$ & 4.83 & 70.58 & 75.91 & \multirow[t]{2}{*}{.57} \\
\hline Other & 21 & 0.41 & $0.22 ; 0.61$ & 4.16 & 58.31 & 65.70 & \\
\hline
\end{tabular}

Note. CAU, Care as usual; CBT, cognitive behavioural therapy; CI, confidence interval; ES, effect size (Hedges' g); IPT, interpersonal psychotherapy; MDD, major depressive disorder. Analyses were conducted according to the random effects model; $\mathrm{p}$ values of the differences between subgroups are based on fixed effects 


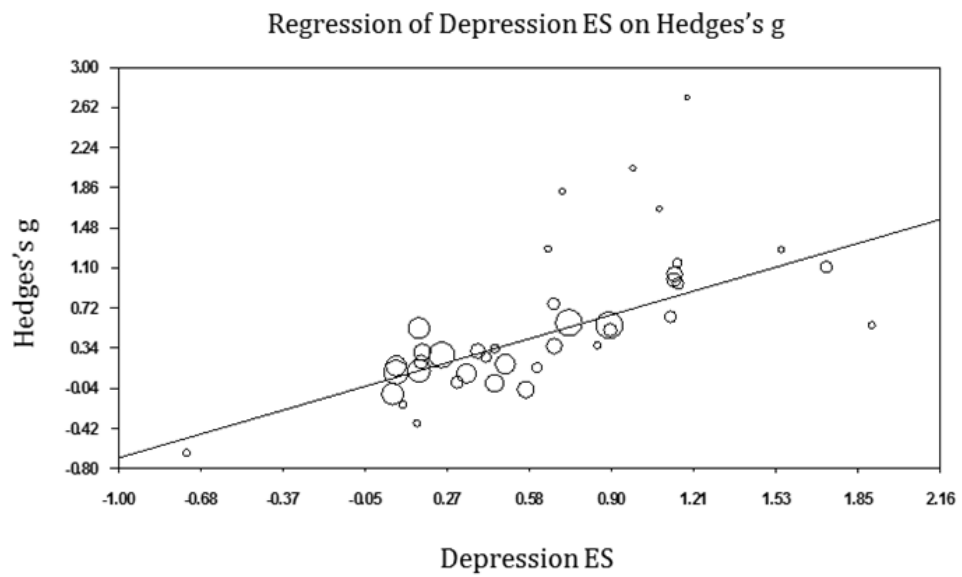

Figure 3. Relation between effect size for depressive symptom severity and effect size for social functioning. Meta-regression analysis.

\section{Multivariate Analyses}

We conducted a series of multivariate meta-analyses with the effect size of social functioning as dependent variable and the effect size of depression severity as predictor. Additionally, we entered the same variables that were used in the univariate subgroup analyses as predictors. First a full model, including all potential predictors was computed. We then removed the least significant predictor from the model until a model with only significant predictors remained (backward deletion). The results of these analyses are reported in Table 3.

In the full model, including all predictors, the effect size of depression severity was a significant predictor of the effect of psychotherapy on social functioning, $b=0.93 ; 95 \%$ CI:0.61; $1.25, p<.001$. The other predictors were not statistically significantly related to the effect size of psychotherapy for social functioning. In the model including all predictors, the effect of psychotherapy on social functioning (constant) was not significant $(\mathrm{p}=.49)$.

Next, non-significant predictors were removed step-for-step, until only significant predictors remained. After removing non-significant predictors step-for-step, the effect size of depressive symptom severity remained as a significant predictor of the effect size of social functioning ( $b=0.81 ; 95 \% \mathrm{CI}: 0.59 ; 1.03, p<.001)$, indicating that stronger effects of psychotherapy on depressive symptom severity were positively associated with stronger effects of psychotherapy on social functioning. Moreover, the treatment delivery format significantly predicted the effect size on social functioning $(b=-0.32 ; 95 \%$ CI:-0.56; $-0.09, p<.01$ ), indicating that studies that used individual treatment settings reported stronger effects than studies that used a group psychotherapy format. The number of treatment sessions was also statistically significantly associated with the effect size of social functioning ( $b=0.03 ; 95 \% \mathrm{CI}: 0.01 ; 0.06, p=.01)$, indicating that the effect size increased by 0.03 with each additional treatment session. In the final model, the effect of psychotherapy on social functioning (constant) remained marginally 
significant ( $b=-0.27 ; 95 \% \mathrm{CI}:-0.55 ; 0.01, p=.055)$, indicating that on the one hand improvements in social functioning in psychotherapy for depression are strongly related to improvements in depressive symptom severity while on the other hand social functioning improves at least in part independent from depressive symptoms.

Table 3. Regression coefficients of selected study characteristics predicting the effect size of social functioning: Multivariate meta-regression analyses.

\begin{tabular}{|c|c|c|c|c|c|c|c|c|}
\hline & \multicolumn{4}{|c|}{ Full Model } & \multicolumn{4}{|c|}{ Final Model } \\
\hline & $\mathrm{B}$ & $95 \% \mathrm{CI}$ & SE & $\mathrm{p}$ & B & $95 \% \mathrm{CI}$ & SE & $\mathrm{p}$ \\
\hline ES depression & 0.93 & $0.61 ; 1.25$ & 0.15 & $<.001$ & 0.81 & $0.59 ; 1.03$ & 0.11 & $<.001$ \\
\hline Format & -0.32 & $-0.77 ; 0.13$ & 0.22 & 0.15 & -0.32 & $-0.56 ; 0.09$ & 0.12 & $<.01$ \\
\hline \#Sessions & 0.04 & $-0.04 ; 0.11$ & 0.04 & 0.32 & 0.03 & $0.01 ; 0.06$ & 0.01 & 0.01 \\
\hline IPT vs. other & -0.19 & $-0.63 ; 0.25$ & 0.21 & 0.37 & - & - & - & - \\
\hline CBT vs. other & -0.17 & $-0.58 ; 0.24$ & 0.20 & 0.40 & - & - & - & - \\
\hline Type Instrument & 0.20 & $-0.58 ; 0.98$ & 0.37 & 0.60 & - & - & - & - \\
\hline Gender & -0.10 & $-0.55 ; 0.34$ & 0.21 & 0.64 & - & - & - & - \\
\hline Quality & 0.04 & $-0.16 ; 0.23$ & 0.09 & 0.70 & - & - & - & - \\
\hline Def. Depression & -0.06 & $-0.49 ; 0.38$ & 0.21 & 0.79 & - & - & - & - \\
\hline Target Group & -0.05 & $-0.49 ; 0.38$ & 0.21 & 0.80 & - & - & - & - \\
\hline Type Control & -0.01 & $-0.45 ; 0.43$ & 0.21 & 0.96 & - & - & - & - \\
\hline Country & -0.00 & $-0.48 ; 0.47$ & 0.23 & 0.99 & - & - & - & - \\
\hline Constant & -0.31 & $-1.24 ; 0.61$ & 0.44 & 0.49 & -0.27 & $-0.55 ; 0.01$ & 0.14 & .055 \\
\hline
\end{tabular}

Note. CBT, Cognitive behavioural therapy; CI, confidence interval; ES, effect size; IPT, interpersonal psychotherapy; S.E., standard error. To build the final model the least significant variable was dropped in a backwards regression analyses until only significant predictors remained. Additional analyses that were entered in the full model were: type of control group, format (group v. individual), country where the trial was conducted, type of instrument used (self-report v. clinician rated), number of treatment sessions, type of diagnosis [DSM-IV-based major depressive disorder (MDD)v. cut-off on depression questionnaire], target group (older adults v. adults), gender (females only v. mixed groups), type of treatment (CBT v. other and IPT v. other), quality of the trial. None of these characteristics were significantly related to the effect size of change in social-interpersonal functioning in the multivariate analyses (all $p$ values $>0.05$ ).

\section{Discussion}

The aim of this meta-analysis was to summarize the effect of psychotherapy for depression on improvements in social functioning. We found 31 studies with a total of 2,956 participants that compared psychotherapy for depression with a control group and assessed social functioning at post-treatment. Before and after adjusting for publication bias, the overall effect size was small to moderate. These findings suggest that depressed patients receiving psychotherapy for depression can benefit not only in 
terms of amelioration of depressive symptoms but also in terms of improved functioning in various social roles such as work, recreational and interpersonal relations. This is important since impairments in these areas strongly contribute to the burden of disease in depression (Eaton et al., 2008, Greenberg et al., 2003). After controlling for the effects of psychotherapy on depressive symptom severity and selective study characteristic in multivariate meta-analyses, the effects of psychotherapy on social functioning remained marginally significant, indicating that changes in social functioning are not fully explained by changes in depressive symptom severity. In other words, our results suggest that social functioning improves as depressive symptoms improve, although the direction or nature of the presumed causal relation remains unknown. These findings are in line with previous studies showing that changes in psychosocial functioning are intercorrelated with changes in depressive symptom severity (e.g. Dunn et al., 2012, Vittengl et al., 2004).

It should be noted that the current research design does not allow determining the temporal relation of this association. It is not clear whether improvements in depressive symptom severity drive improvements in social functioning or the other way around. To determine the temporal relation between these constructs it would be necessary to establish a timeline with repeated assessments of both constructs during treatment (Kazdin, 2007). One previous process-outcome study on the effects of cognitive therapy on change in psychosocial adjustment found that improvements in psychosocial adjustment, assessed four times during treatment, predicted subsequent reductions in depressive symptoms rather than the other way around (Dunn et al., 2012). Future RCT based studies with control groups and repeated assessments of functioning and depressive symptoms throughout treatment should aim to determine the causal relation between these variables.

Overall, the effect size of social functioning was smaller than the effect size of depressive symptom severity. This finding is in line with previous studies showing that impairments in social functioning often persist, even after symptomatic recovery (e.g. Coryell et al., 1993). Different explanations might account for this finding. For example, it is possible that social functioning takes more time to improve compared to depressive symptom severity in psychotherapy for depression. This explanation is in line with the findings of a previous meta-analysis showing that recovery from work impairments in depression takes longer than symptom remission (Mintz et al., 1992). On the other hand, impairments in social functioning might be more persistent even in the long run and additional treatment might be necessary to improve social functioning to a premorbid level. Our finding that social functioning improved less than depressive symptom severity might have important prognostic implications for the course of depression following treatment. For example, it has been shown that residual impairments in social functioning following treatment are related to relapse and recurrences (e.g. Leon et al., 1999, Vittengl et al., 2009). Such findings stress the importance of including social functioning as main outcome in treatment studies and in current definitions of response and remission. It should be noted that after adjusting for publication bias the effect size 
of social functioning ( $g=0.40$ ) was comparable to the adjusted effect size of depressive symptoms $(\mathrm{g}=0.43)$.

We conducted a number of subgroup analyses to test whether selective study characteristics were differentially related to the effects of psychotherapy on social functioning. In interpreting the results of these analyses, it should be noted that significant associations in these analyses should not be interpreted in terms of causal associations. Our univariate subgroup analyses indicated that studies that compared psychotherapy to care-as-usual resulted in smaller effect sizes compared to studies comparing psychotherapy to other control conditions. This finding is in line with previous meta-analyses summarizing the effects of psychotherapy on depressive symptom severity (e.g. Cuijpers et al., 2008d). In our subgroup analyses studies with a care-as-usual control condition were mainly compared to studies with a waiting list control condition and it is therefore not surprising that studies with care-as-usual as control condition resulted in smaller effect sizes. Our subgroup analyses further showed that studies that were conducted in the US and studies that used clinician-rated scales to assess social functioning yielded higher effect sizes compared to studies that were conducted in other countries or studies that relied on self-reported instruments of social functioning. Although speculative, it is possible, that US based studies have a higher treatment delivery quality and therefore achieve higher effect sizes. The finding that clinician-rated instruments yielded higher effect sizes than self-reported instruments is in line with the results of an earlier meta-analysis showing that clinician-rated instruments were associated with higher effect-sizes of depressive symptom severity (Cuijpers et al., 2010). In the current meta-analysis only three studies (seven comparisons) used a clinician-rated instrument of social functioning and hence this finding should be interpreted with caution. Based on our findings it is not clear whether one type of assessment of social functioning is preferable over the other and therefore it is probably best to include both in future studies and clinical practice when assessing social functioning.

There were no differences in improvements in social functioning between different types of psychotherapeutic interventions (CBT versus others; IPT versus others). When interpreting these results it is important to keep in mind that these findings are based on separate sets of comparisons and not on direct comparisons of different types of psychotherapies. Such indirect comparisons might be confounded by differences in patient populations and other study characteristics and the results should therefore be interpreted with caution. Our findings are in line with previous meta-analyses reporting that different types of psychotherapy are approximately equally efficacious in reducing depressive symptoms (Cuijpers et al., 2008a). IPT specifically focuses on social functioning (Weissman et al., 2000) and one might therefore expect that studies that included IPT would result in larger effect sizes of improvements in social functioning, compared to studies on other forms of psychotherapy. It is unclear how our finding that improvements in social functioning were not different between IPT studies and other studies relates to the literature on the mechanisms of change in depression. It is possible, for example, that IPT based studies in this meta-analyses resulted in initially stronger 
improvements in social functioning but that these differences were not present anymore at post-treatment. However, it is impossible to test this hypothesis in the current research design. Future RCT based studies with repeated assessments throughout treatment should aim to determine the temporal pattern of improvements in social functioning in different types of psychotherapy for depression.

We found that the number of therapy sessions was positively associates with the effect size of social functioning, even after controlling for the effects on depressive symptom severity in multivariate analyses. This finding suggests that patients with depression might benefit from longer term treatments or continuation treatments in terms of improvements in social functioning. The relation between the number of therapy sessions and the effect size of social functioning was rather small. Our analyses suggest that an increase of 5 therapy sessions is associated with an increase in the effect size of social functioning of 0.15 . This finding is in line with the findings of a recent metaanalysis showing that there is a small association between the number of therapy sessions and the effect size of depressive symptom severity (Cuijpers et al., 2013). In the current meta-analysis studies on continuation and maintenance treatments were excluded and it therefore remains unclear if continued treatment is related to further improvements in social functioning. In the univariate and multivariate meta-analyses we found that the treatment delivery format was significantly associated with improvements in social functioning. Studies that used an individual treatment format resulted in higher effect sizes compared to studies that used a group format. On theoretical grounds one might argue that group settings should result in stronger improvements in social functioning because group therapy by definition takes place in a social interpersonal context allowing, for example, to practice social and interpersonal conflicts in vivo in the group. Empirically, our finding that individual treatments resulted in higher effect sizes of social functioning is in line with the results of a previous metaanalysis showing that an individual treatment format for depression is associated with higher effect sizes of depressive symptom severity (Cuijpers et al., 2008b).

Several important limitations should be mentioned. First, the number of studies was relatively small and accordingly it was not possible to compare effect sizes between specific types of treatment other than CBT and IPT. For example, it would be interesting to test whether psychotherapies for depression that focus on engagement in psychosocial activities (for example behavioural activation) are related to stronger improvements in psychosocial adjustments compared to other forms of psychotherapies. A related limitation of this meta-analysis is that comparisons between different types of psychotherapies were based on separate sets of comparisons and not on direct comparisons of different types of psychotherapies. The number of comparative outcome studies in the current meta-analysis was too small to allow for such a direct comparison. Second, on average the quality of included studies was rather low. Since quality was negatively associated with the effect size, it might be that the effect sizes in the current meta-analysis were overestimated. Third, we compared the effects of psychotherapy versus control groups at one assessment moment and hence we could not draw any conclusions regarding the temporal relations between changes in social functioning and 
changes in depressive symptom severity. The current meta-analysis was based on study level data. Another approach that might also allow for analyses of temporal relations and a more fine-grained analysis of predictors would have been to conduct an individual patient-level meta-analysis based on the original datasets of included studies. Finally, we assessed overall improvements in social functioning rather than domain specific improvements. It is possible, for example, that some aspects of functioning show stronger improvements (e.g. recreational functioning) than others (e.g. work functioning).

Despite these limitations, our findings have important implications for clinical practice and future research. First, the results of this meta-analysis suggest that psychotherapy for depression is associated with improvements in social functioning. This is an important finding, since improvements in social functioning are probably a highly desirable outcome from a patient perspective. Second, future research should aim to determine the temporal relation between social functioning and depressive symptom severity by assessing both depressive symptom severity and social functioning repeatedly throughout treatment. Finally, compared to the total number of RCTs on different types of psychotherapies for depression, the number of studies that included measures of social functioning as treatment outcome was relatively small, which reflects the current focus of treatment outcome studies on depressive symptom severity rather than functioning. Including social functioning as main outcome, in addition to symptomatic improvements, in treatment studies for depression might add to a more complete definition of treatment effects.

In conclusion, psychotherapy for depression has small to moderate positive effects on the social functioning of depressed patients and no differences between different types of psychotherapies emerged.

\section{Declaration of Interest}

None. 


\section{References}

Allart-van Dam, E., Hosman, C. M. H., Hoogduin, C. A. L. \& Schaap, C. P. D. R. (2003). The Coping With Depression course: Short-term outcomes and mediating effects of a randomized controlled trial in the treatment of subclinical depression. Behavior Therapy 34, 381-396.

Barth, J., Munder, T., Gerger, H., Nüesch, E., Trelle, S., Znoj, H., Jüni, P. \& Cuijpers, P. (2013). Comparative Efficacy of Seven Psychotherapeutic Interventions for Patients with Depression: A Network MetaAnalysis. PLoS Med 10, e1001454.

Battle, C. L., Uebelacker, L., Friedman, M. A., Cardemil, E. V., Beevers, C. G. \& Miller, I. W. (2010). Treatment Goals of Depressed Outpatients: A Qualitative Investigation of Goals Identified by Participants in a Depression Treatment Trial. Journal of Psychiatric Practice ${ }^{\circledR}$ 16, 425-430 10.1097/01.pra.0000390763.57946.93.

Berger, T., Hämmerli, K., Gubser, N., Andersson, G. \& Caspar, F. (2011). Internet-based treatment of depression: A randomized controlled trial comparing guided with unguided self-help. Cognitive Behaviour Therapy 40, 251-266.

Bosc, M. (2000). Assessment of social functioning in depression. Comprehensive Psychiatry 41, 63-69.

Choi, I., Zou, J., Titov, N., Dear, B. F., Li, S., Johnston, L., Andrews, G. \& Hunt, C. (2012). Culturally attuned Internet treatment for depression amongst Chinese Australians: A randomised controlled trial. Journal of Affective Disorders 136, 459-468.

Coryell, W., Scheftner, W., Keller, M. \& Endicott, J. (1993). The enduring psychosocial consequences of mania and depression. The American Journal of Psychiatry 150, 720-727.

Cuijpers, P., Huibers, M., Daniel Ebert, D., Koole, S. L. \& Andersson, G. (2013). How much psychotherapy is needed to treat depression? A metaregression analysis. Journal of Affective Disorders 149, 1-13.

Cuijpers, P., Li, J., Hofmann, S. G. \& Andersson, G. (2010). Self-reported versus clinician-rated symptoms of depression as outcome measures in psychotherapy research on depression: A meta-analysis. Clinical Psychology Review 30, 768-778.

Cuijpers, P., van Straten, A., Andersson, G. \& van Oppen, P. (2008a). Psychotherapy for depression in adults: A meta-analysis of comparative outcome studies. Journal of Consulting and Clinical Psychology 76, 909-922.

Cuijpers, P., van Straten, A. \& Warmerdam, L. (2008b). Are individual and group treatments equally effective in the treatment of depression in adults? A meta-analysis. The European Journal of Psychiatry 22, 38-51.

Cuijpers, P., van Straten, A., Warmerdam, L. \& Andersson, G. (2008c). Psychological treatment of depression: A meta-analytic database of randomized studies. BMC Psychiatry 8.

Cuijpers, P., Van Straten, A., Warmerdam, L. \& Smits, N. (2008d). Characteristics of effective psychological treatments of depression: A metaregression analysis. Psychotherapy Research 18, 225-236.

Dobkin, R. D., Menza, M., Allen, L. A., Gara, M. A., Mark, M. H., Tiu, J., Bienfait, K. L. \& Friedman, J. (2011). Cognitive-behavioral therapy for depression in Parkinson's disease: A randomized, controlled trial. The American Journal of Psychiatry 168, 1066-1074.

Dowrick, C., Dunn, G., Ayuso-Mateos, J. L., Dalgard, O. S., Page, H., Lehtinen, V., Casey, P., Wilkinson, C., Vazquez-Barquero, J. L. \& Wilkinson, G. (2000). Problem solving treatment and group psychoeducation for depression: Multicentre randomised controlled trial. BMJ: British Medical Journal 321.

Dunn, T. W., Vittengl, J. R., Clark, L. A., Carmody, T., Thase, M. E. \& Jarrett, R. B. (2012). Change in psychosocial functioning and depressive symptoms during acute-phase cognitive therapy for depression. Psychological Medicine 42, 317-326.

Duval, S. \& Tweedie, R. (2000). Trim and Fill: A Simple Funnel-Plot-Based Method of Testing and Adjusting for Publication Bias in Meta-Analysis. Biometrics 56, 455-463.

Eaton, W. W., Martins, S. S., Nestadt, G., Bienvenu, O. J., Clarke, D. \& Alexandre, P. (2008). The Burden of Mental Disorders. Epidemiologic Reviews 30, 1-14. 
Ekers, D., Richards, D., McMillan, D., Bland, J. M. \& Gilbody, S. (2011). Behavioural activation delivered by the nonspecialist: Phase II randomised controlled trial. The British Journal of Psychiatry 198, 66-72.

Faramarzi, M., Kheirkhah, F., Esmaelzadeh, S., Alipour, A., Hjiahmadi, M. \& Rahnama, J. (2008). Is psychotherapy a reliable alternative to pharmacotherapy to promote the mental health of infertile women? A randomized clinical trial. European Journal of Obstetrics \&amp; Gynecology and Reproductive Biology 141, 49-53.

Fledderus, M., Bohlmeijer, E. T., Pieterse, M. E. \& Schreurs, K. M. G. (2012). Acceptance and commitment therapy as guided self-help for psychological distress and positive mental health: a randomized controlled trial. Psychological Medicine 42, 485-495.

Greenberg, P. E., Kessler, R. C., Birnbaum, H. G., Leong, S. A., Lowe, S. W., Berglund, P. A. \& Corey-Lisle, P. K. (2003). The economic burden of depression in the United States: How did it change between 1990 and 2000? Journal of Clinical Psychiatry 64, 1465-1475.

Grote, N. K., Swartz, H. A., Geibel, S. L., Zuckoff, A., Houck, P. R. \& Frank, E. (2009). A randomized controlled trial of culturally relevant, brief interpersonal psychotherapy for perinatal depression. Psychiatr Serv 60, 313-21.

Haringsma, R., Engels, G. I., Cuijpers, P. \& Spinhoven, P. (2006). Effectiveness of the Coping With Depression (CWD) course for older adults provided by the community-based mental health care system in the Netherlands: A randomized controlled field trial. International Psychogeriatrics 18, 307-325.

Harley, R., Sprich, S., Safren, S., Jacobo, M. \& Fava, M. (2008). Adaptation of dialectical behavior therapy skills training group for treatment-resistant depression. Journal of Nervous and Mental Disease 196, 136-143.

Hays, R. D., Wells, K. B., Sherbourne, C., Rogers, W. \& Spritzer, K. (1995). FUnctioning and well-being outcomes of patients with depression compared with chronic general medical illnesses. Archives of General Psychiatry 52, 11-19.

Hedges, L. V. \& Olkin, I. (1985). Statistical Methods for Meta-Analysis. Academic Press: San Diego, CA.

Higgins, J. P. T., Altman, D. G., Gøtzsche, P. C., Jüni, P., Moher, D., Oxman, A. D., Savović, J., Schulz, K. F., Weeks, L. \& Sterne, J. A. C. (2011). The Cochrane Collaboration's tool for assessing risk of bias in randomised trials. $B M J 343$.

Higgins, J. P. T., Thompson, S. G., Deeks, J. J. \& Altman, D. G. (2003). Measuring inconsistency in metaanalyses. BMJ: British Medical Journal 327, 557-560.

Hirschfeld, R. M. A., Montgomery, S. A., Keller, M. B., Kasper, S., Schatzberg, A. F., Möller, H.-J., Healy, D., Baldwin, D., Humble, M., Versiani, M., Montenegro, R. \& Bourgeois, M. (2000). Social functioning in depression: A review. Journal of Clinical Psychiatry 61, 268-275.

Horowitz, L. M., Alden, L. E., Wiggins, J. S. \& Pincus, A. L. (2000). Manual for the Inventory of Interpersonal Problems. Harcourt Assessment The Psychological Corporation: Oxford, UK.

Judd, L. L., Akiskal, H. S., Zeller, P. J., Paulus, M., Leon, A. C., Maser, J. D., Endicott, J., Coryell, W., Kunovac, J. L., Mueller, T. I., Rice, J. P. \& Keller, M. B. (2000). Psychosocial disability during the long-term course of unipolar major depressive disorder. Archives of General Psychiatry 57, 375-380.

Kazdin, A. E. (2007). Mediators and mechanisms of change in psychotherapy research. Annual Review of Clinical Psychology 3, 1-27.

Klein, M. H., Greist, J. H., Gurman, A. S., Neimeyer, R. A., Lesser, D. P., Bushnell, N., J. \& Smith, R. E. (1985). A comparative outcome study of group psychotherapy vs. exercise treatments for depression. International Journal of Mental Health 13, 148-177.

Laidlaw, K., Davidson, K., Toner, H., Jackson, G., Clark, S., Law, J., Howley, M., Bowie, G., Connery, H. \& Cross, S. (2008). A randomised controlled trial of cognitive behaviour therapy vs treatment as usual in the treatment of mild to moderate late life depression. International Journal of Geriatric Psychiatry 23, 843-850.

Leon, A. C., Solomon, D. A., Mueller, T. I., Turvey, C. L., Endicott, J. \& Keller, M. B. (1999). The Range of Impaired Functioning Tool (LIFE-RIFT): a brief measure of functional impairment. Psychological Medicine 29, 869-878. 
Lynch, D., Tamburrino, M., Nagel, R. \& Smith, M. K. (2004). Telephone-based Treatment For Family Practice Patients With Mild Depression. Psychological Reports 94, 785-792.

Lynch, D. J., Tamburrino, M. B. \& Nagel, R. (1997). Telephone counseling for patients with minor depression: Preliminary findings in a family practice setting. The Journal of Family Practice 44, 293298.

Miller, L. \& Weissman, M. (2002). Interpersonal psychotherapy delivered over the telephone to recurrent depressives : A pilot study. Depression and Anxiety 16, 114-117.

Mintz, J., Mintz, L. I., Arruda, M. J. \& Hwang, S. S. (1992). Treatments of depression and the functional capacity to work. Arch Gen Psychiatry 49, 761-8.

Miranda, J., Chung, J. Y., Green, B. L., Krupnick, J., Siddique, J., Revicki, D. A. \& Belin, T. (2003). Treating Depression in Predominantly Low-Income Young Minority Women: A Randomized Controlled Trial. JAMA: Journal of the American Medical Association 290, 57-65.

Mynors-Wallis, L. M., Gath, D. H., Lloyd-Thomas, A. R. \& Tomlinson, D. (1995). Randomised controlled trial comparing problem solving treatment with amitriptyline and placebo for major depression in primary care. $B M J 310,441-445$.

Neugebauer, R., Kline, J., Markowitz, J. C., Bleiberg, K. L., Baxi, L., Rosing, M. A., Levin, B. \& Keith, J. (2006). Pilot Randomized Controlled Trial of Interpersonal Counseling for Subsyndromal Depression Following Miscarriage. Journal of Clinical Psychiatry 67, 1299-1304.

O'Hara, M. W., Stuart, S., Gorman, L. L. \& Wenzel, A. (2000). Efficacy of interpersonal psychotherapy for postpartum depression. Archives of General Psychiatry 57, 1039-1045.

Office of National Statistics (2012). Sickness Absence in the Labour Market, April 2012.

Perini, S., Titov, N. \& Andrews, G. (2009). Clinician-Assisted Internet-Based Treatment is Effective for Depression: Randomized Controlled Trial. Australian and New Zealand Journal of Psychiatry 43, 571-578.

Propst, L. R., Ostrom, R., Watkins, P., Dean, T. \& Mashburn, D. (1992). Comparative efficacy of religious and nonreligious cognitive-behavioral therapy for the treatment of clinical depression in religious individuals. Journal of Consulting and Clinical Psychology 60, 94-103.

Scott, J., Teasdale, J. D., Paykel, E. S., Johnson, A. L., Abbott, R., Hayhurst, H., Moore, R. \& Garland, A. (2000). Effects of cognitive therapy on psychological symptoms and social functioning in residual depression. The British Journal of Psychiatry 177, 440-446.

Serfaty, M. A., Haworth, D., Blanchard, M., Buszewicz, M., Murad, S. \& King, M. (2009). Clinical effectiveness of individual cognitive behavioral therapy for depressed older people in primary care: A randomized controlled trial. Archives of General Psychiatry 66, 1332-1340.

Sheehan, D. (1983). The Anxiety Disease. Scribner's: New York.

Simpson, S., Corney, R., Fitzgerald, P. \& Beecham, J. (2003). A randomized controlled trial to evaluate the effectiveness and cost-effectiveness of psychodynamic counselling for general practice patients with chronic depression. Psychological Medicine 33, 229-239.

Solomon, D. A., Leon, A. C., Endicott, J., Mueller, T. I., Coryell, W., Shea, M. T. \& Keller, M. B. (2004). Psychosocial Impairment and Recurrence of Major Depression. Comprehensive Psychiatry 45, 423430.

Talbot, N. L., Chaudron, L. H., Ward, E. A., Duberstein, P. R., Conwell, Y., O'Hara, M. W., Tu, X., Lu, N., He, H. \& Stuart, S. (2011). A randomized effectiveness trial of interpersonal psychotherapy for depressed women with sexual abuse histories. Psychiatr Serv 62, 374-80.

van Schaik, A., van Marwijk, H., Adèr, H., van Dyck, R., de Haan, M., Penninx, B., van der Kooij, K., van Hout, H. \& Beekman, A. (2006). Interpersonal Psychotherapy for Elderly Patients in Primary Care. The American Journal of Geriatric Psychiatry 14, 777-786.

Vitriol, V. G., Ballesteros, S. T., Florenzano, R. U., Weil, K. P. \& Benadof, D. F. (2009). Evaluation of an outpatient intervention for women with severe depression and a history of childhood trauma. Psychiatric Services 60, 936-942.

Vittengl, J. R., Clark, L. A. \& Jarrett, R. B. (2004). Improvement in social-interpersonal functioning after cognitive therapy for recurrent depression. Psychological Medicine 34, 643-658. 
Vittengl, J. R., Clark, L. A. \& Jarrett, R. B. (2009). Deterioration in psychosocial functioning predicts relapse/recurrence after cognitive therapy for depression. Journal of Affective Disorders 112, 135143.

Ward, E., King, M., Lloyd, M., Bower, P., Sibbald, B., Farrelly, S., Gabbay, M., Tarrier, N. \& Addington-Hall, J. (2000). Randomised controlled trial of non-directive counselling, cognitive-behaviour therapy, and usual general practitioner care for patients with depression. I: Clinical effectiveness. BMJ: British Medical Journal 321, 1383-1388.

Ware, J. E., Jr. \& Sherbourne, C. D. (1992). The MOS 36-item short-form health survey (SF-36). I. Conceptual framework and item selection. Med Care 30, 473-83.

Watkins, J. T., Leber, W. R., Imber, S. D., Collins, J. F., Elkin, I., Pilkonis, P. A., Sotsky, S. M., Shea, M. T. \& Glass, D. R. (1993). Temporal course of change of depression. Journal of Consulting and Clinical Psychology 61, 858-864.

Watt, L. M. \& Cappeliez, P. (2000). Integrative and instrumental reminiscence therapies for depression in older adults: Intervention strategies and treatment effectiveness. Aging and Mental Health 4, 166177.

Weissman, M., Markowitz, J. \& Klerman, G. (2000). Comprehensive Guide to interpersonal Psychotherapy. Basic Books: New York.

Weissman, M. M., Prusoff, B. A., Thompson, W. D., Harding, P. S. \& Myers, J. K. (1978). Social adjustment by self-report in a community sample and in psychiatric outpatients. J Nerv Ment Dis 166, 317-26.

World Health Organization (1948). Preamble to the Constitution of the World Health Organization as adopted by the International Health Conference, New York, 19-22 June, 1946.

Zimmerman, M., McGlinchey, J. B., Posternak, M. A., Friedman, M., Attiullah, M. \& Boerescu, D. (2006). How Should Remission From Depression Be Defined? The Depressed Patient's Perspective. The American Journal of Psychiatry 163, 148-150. 


\section{PART III}

\section{SCHEMA THERAPY FOR CHRONIC DEPRESSION}





\section{CHAPTER 8 \\ Schema therapy for chronic depression: Rationale}

Published as:

Renner, F., Arntz, A., Leeuw, I., \& Huibers, M. (2013). Treatment for chronic depression using schema therapy. Clinical Psychology Science and Practice, 20, 166-180. 


\begin{abstract}
Schema Therapy (ST) is an integrative treatment approach to chronic lifelong problems with an established effectiveness for treating personality disorders. This article describes the adaptation of ST to chronic depression by reviewing the literature on the underlying risk factors to chronic depression. A model of chronic depression is presented, describing the interplay between empirically supported risk factors to chronic depression (early adversity, cognitive factors, personality pathology, interpersonal factors). We provide a treatment protocol of ST for chronic depression describing techniques that can be used in ST to target these underlying risk factors. Based on the current body of empirical evidence for the underlying risk factors to chronic depression, ST appears to be a promising new treatment approach to chronic depression as it directly targets these underlying risk factors.
\end{abstract}

Keywords: Chronic depression, Schema therapy, Early maladaptive schemas, Risk factors 


\section{Introduction}

Schema Therapy (ST) is an integrative treatment approach combining cognitive, behavioral, experiential and psychodynamic elements and techniques (Young, Klosko, \& Weishaar, 2003). Influenced by the cognitive theory of depression, Young elaborated on the schema concept and developed ST as a novel treatment for clients with chronic psychological disorders. Empirical research on the effectiveness of ST is in its initial stage. While there is evidence for the effectiveness of ST in treating clients with borderline personality disorder (Farrell, Shaw, \& Webber, 2009; Giesen-Bloo et al., 2006; Nadort et al., 2009; Nordahl, Holthe, \& Haugum, 2005) and clients with Cluster-C, paranoid, histrionic and narcissistic personality disorders (Bamelis, Evers, Spinhoven, \& Arntz, 2012) there is a lack of research on ST for chronic axis-I disorders. In addition to the effects of ST on personality pathology, a recent large randomized controlled trial found that ST also has positive effects on co-morbid depression in clients with primarily Cluster-C personality disorders (Bamelis, et al., 2012). Such findings raise the question whether ST is also a valuable treatment for clients with primary depressive symptoms.

Chronic depression is a relatively common (affecting 19\% of depressed clients; Keller, Hanks, Kocsis, \& Klein, 1995) and difficult to treat (Kocsis, 2003; Riso \& Newman, 2003) mental disorder. Four types of chronic depression are distinguished in the literature: chronic major depressive disorder, dysthymic disorder, double depression, and recurrent major depressive disorder (MDD) without full interepisode recovery (Klein, 2010). Whereas these subtypes appear to be rather homogenous with respect to etiology and clinical features, they can be clinically distinguished from episodic forms of depression (McCullough et al., 2003). Compared to episodic forms of depression, chronic depression has a stronger negative impact on the quality of life (Wells, Burnam, Rogers, \& Hays, 1992), results in more suicide attempts and hospitalizations (Arnow \& Constantino, 2003; Torpey \& Klein, 2008), is associated with greater service use (Howland, 1993), and higher economic costs (Smit et al., 2006), stressing the importance of effective treatment protocols for chronic forms of depression.

\section{Treatment of Chronic Depression}

Common treatment approaches to chronic depression include antidepressant medication (ADM; Kocsis, 2003), cognitive therapy (CT; Riso \& Newman, 2003), cognitive behavioral analysis system of psychotherapy (CBASP; McCullough, 2003), and combined psychotherapy and medication treatments (Thase, 1999). Although psychotherapy and ADM treatments have an established effectiveness in the treatment of chronic depression, the effect sizes of psychotherapy in reducing depressive symptoms are small (Cuijpers et al., 2010) and remission rates of ADM treatment for chronic depression usually fall well below 50\% (Kocsis, 2003), reflecting the need for more effective treatment approaches for chronic depression. Higher response and remission rates are obtained in ADM and psychotherapy combination treatments (Browne et al., 2002; Keller et al., 2000; Manber et al., 2008; Pampallona, Bollini, Tibaldi, 
Kupelnick, \& Munizza, 2004; Thase et al., 1997). For example, in a large randomized clinical trial (RCT) $\mathrm{N}=681$ outpatients with chronic depression were randomized to either ADM treatment (nefazodone), CBASP, or a combination treatment (Keller, et al., 2000). In line with earlier studies of psychotherapy or ADM treatment for chronic depression, about half (48\%) of the clients in the ADM and CBASP alone groups responded to treatment. In the combination group more than two thirds of the clients (73\%) responded to treatment (Keller, et al., 2000) which is in line with the notion that combined treatments for chronic depression results in more positive outcomes (Pampallona, et al., 2004).

Although these results seem promising, the initial effects of treatments for chronic depression are often not maintained and continuation phase treatment (4-9 months) and/or maintenance phase treatments ( 1 year or more) are necessary to reduce relapse and recurrences. For example, Gelenberg et al. (2003) randomized outpatients with chronic depression, who achieved and maintained a clinical response during acutephase and continuation phase treatment for chronic depression, to either 52 weeks of nefazodone (Selective Serotonin Reuptake Inhibitor; SSRI) or pill placebo. The authors found that the risk for recurrence at the end of the 1 year maintenance phase was statistically significantly lower in the nefazodone group (30.3\%) compared to the placebo group (47.5\%). Yet, clients who were initially treated to remission (in the acute phase of the same study) with CBASP did not show lower relapse rates compared to clients who were treated to remission with nefazodone. These findings suggest that CBASP does not add any prophylactic effects above ADM in the treatment of chronic depression (Gelenberg, et al., 2003). Moreover, augmentation with CBASP to continued ADM treatment in chronically depressed non-responders has been shown to have no beneficial effects above continued ADM treatment alone (Kocsis et al., 2009). Finally, combination treatment of CBASP and ADM in the acute phase seems to be superior to either treatment alone only for a subgroup of moderately depressed clients but not for chronically depressed clients with more severe depressions (Stulz, Thase, Klein, Manber, \& Crits-Christoph, 2010), stressing the need of exploring new treatment options for chronically depressed clients.

CBASP is the only type of psychotherapy that was specifically developed for the treatment of chronic depression and therefore might be superior to other types of psychotherapy used in chronic depression. In a recent study, clients with early-onset chronic depression were randomized to either CBASP or interpersonal psychotherapy (IPT) (Schramm et al., 2011). Although remission rates were remarkably higher in the CBASP group (57\%) compared to the IPT group $(20 \%)$ these effects could not be maintained at a one-year follow-up assessment, suggesting that the initial effects of CBASP are not enduring and that chronically depressed clients did not profit more from CBASP compared to IPT in the long run.

One important reason for the lack of enduring effects of current treatment approaches for chronic depression, including CBASP, might be the relatively small number of treatment sessions. For example, in the study by Keller et al. (2000), chronically depressed outpatients received 16 - 20 sessions of CBASP with a mean 
number of 16 sessions. In a recent meta-analysis of psychotherapy for chronic depression it has been shown that the effects of psychotherapy for chronic depression increase in a dose-response relationship with the number of therapy sessions (Cuijpers, et al., 2010). It is therefore likely that a longer course of psychotherapy is necessary to reach higher levels of remission at follow-up in the treatment of chronic depression.

Another treatment approach to depression that should be mentioned is emotion focused therapy (EFT; Greenberg \& Watson, 2006). EFT is an integrative treatment approach based on client centered, experiential, and cognitive techniques with established evidence as a treatment of acute depression (Ellison, Greenberg, Goldman, \& Angus, 2009; Watson, Gordon, Stermac, Kalogerakos, \& Steckley, 2003). EFT is concerned with identifying and changing emotional structures (emotional schemes) that consist of emotions, cognitions, sensations and behavioral tendencies. Results from process-outcome studies suggest that emotional processing is indeed an important predictor of therapy outcome in depression (Castonguay, Goldfried, Wiser, Raue, \& Hayes, 1996; Watson \& Bedard, 2006). This finding suggests that treatment for (chronic) depression might benefit from a focus on emotional processing in addition to interpersonal and cognitive interventions.

Taken together, while current treatment protocols for chronic depression are effective in treating clients to initial remission, these effects are only maintained with extensive continuation and maintenance treatments. Moreover, the response rates and effect sizes for current ADM and psychotherapeutic treatments for chronic depression, including CT and CBASP, are rather small, stressing the need to explore novel, long-term treatment options for this group of clients. Therefore, the aims of this article are to discuss the theoretical application of a promising new long-term treatment approach to chronic depression, schema therapy (ST), and to provide an overview of a treatment protocol of ST for chronic depression that is currently being tested in a single-case series.

\section{Basic Concepts of Schema Therapy}

\section{Early Maladaptive Schemas}

According to the original ST model, Early Maladaptive Schemas (EMS's) are at the core of psychopathology (Young, et al., 2003). EMS are stable, trait-like, enduring beliefs about oneself and the world that are rooted in early adverse childhood experiences (Young, et al., 2003). Adverse childhood experiences include failure to meet the basic needs of the child, traumatic events, over nurturance, or a strong internalization of parents dysfunctional thoughts, feelings and behaviors. For example, children of overly nurturant parents might develop a strong dependence schema. EMS are in many ways comparable to the concept of core beliefs in CT (Clark \& Beck, 1999). However, there are also important differences between these two concepts (James, Southam, \& Blackburn, 2004). Whereas core beliefs in depression are usually divided into three broad categories (helplessness, inadequacy, and unloveability) EMSs are more specific. To date, 18 specific EMSs were identified and divided into five broader categories (see Table 1). 
According to the ST model, EMS remain latent until triggered by life events and once triggered they can evoke powerful emotional reactions in the individual. For example, a student with a strong failure schema might experience strong feelings of sadness, worthlessness and guilt after failing an exam. Given that EMS are assumed to be at the core of psychopathology, change from maladaptive to more adaptive schemas and coping responses is the ultimate goal of ST.

Table 1. A brief description of all 18 early maladaptive schemas according to Young et al. (2003).

\begin{tabular}{|c|c|}
\hline Early Maladaptive Schema & Description \\
\hline Abandonment / Instability & The perceived instability or unreliability of those available for support. \\
\hline Mistrust / Abuse & $\begin{array}{l}\text { The expectation that others will intentionally hurt, abuse, humiliate, } \\
\text { cheat, lie, manipulate or take advantage. }\end{array}$ \\
\hline Emotional Deprivation & $\begin{array}{l}\text { The expectation that one's desire for emotional support, nurturance, } \\
\text { empathy or protection by others will not be met. }\end{array}$ \\
\hline Defectiveness / Shame & The feeling that one is defective, bad, unwanted, inferior, or invalid. \\
\hline Social Isolation & $\begin{array}{l}\text { The feeling that one is isolated from the world, different from others and } \\
\text { not part of a community. }\end{array}$ \\
\hline Dependence / Incompetence & $\begin{array}{l}\text { The belief that one is not able to handle everyday responsibilities } \\
\text { without help from others. }\end{array}$ \\
\hline Vulnerability to Harm or Illness & $\begin{array}{l}\text { Exaggerated fear that an unpreventable medical, emotional or external } \\
\text { catastrophe will strike. }\end{array}$ \\
\hline Enmeshment & $\begin{array}{l}\text { Excessive emotional involvement with significant others at the expense } \\
\text { of individualization }\end{array}$ \\
\hline Failure & The belief that one has failed or will fail in areas of achievement. \\
\hline Entitlement & $\begin{array}{l}\text { The belief that one is superior to others and entitled to special rights and } \\
\text { privileges. }\end{array}$ \\
\hline Insufficient Self-Control & $\begin{array}{l}\text { A pervasive difficulty or refusal to exercise sufficient self-control and } \\
\text { frustration tolerance to achieve personal goals. }\end{array}$ \\
\hline Subjugation & Surrendering of control to others to avoid negative consequences. \\
\hline Self-Sacrifice & $\begin{array}{l}\text { The excessive focus of meting needs of others at the expense of one's } \\
\text { own gratification. }\end{array}$ \\
\hline Approval-Seeking & $\begin{array}{l}\text { An excessive focus on gaining approval, recognition, or attention from } \\
\text { others. }\end{array}$ \\
\hline Negativity & $\begin{array}{l}\text { A lifelong focus on the negative aspects of life while minimizing the } \\
\text { positive aspects. }\end{array}$ \\
\hline Emotional Inhibition & $\begin{array}{l}\text { Inhibiting spontaneous action, feelings, or communication to avoid } \\
\text { disapproval by others or feelings of shame. }\end{array}$ \\
\hline Unrelenting Standards & $\begin{array}{l}\text { The belief that one must strive to meet very high standards to avoid } \\
\text { criticism. }\end{array}$ \\
\hline Punitiveness & The belief that people should be harshly punished for mistakes. \\
\hline
\end{tabular}




\section{Coping Strategies}

Clients with strong EMS develop dysfunctional coping strategies in order to cope with or compensate for the negative emotions that they might experience when schemas are activated (Young, et al., 2003). In the schema model three types of coping responses are described: (1) schema avoidance (behaviors aimed at avoiding activation of the schema), (2) schema surrender (behaviors that confirm the schema), and (3) schema overcompensation (behaviors that are opposite to the schema). These coping strategies are maladaptive because they maintain the underlying schema. For example, clients with a strong mistrust and abuse schema might engage in schema surrender coping by being drawn into abusive relationships, thereby confirming the validity of their schema.

\section{Adaptation of ST to chronic depression}

Like other mental disorders, chronic depression is unlikely to be caused and maintained by a single underlying factor, but likely to arise from a set of multiple interacting factors. Since the introduction of chronic depression as a separate category in the DSM-III a number of studies have been conducted to determine underlying risk factors to chronic depression by comparing clients with episodic forms of depression to chronically depressed clients. While there is now evidence for a number of developmental and cognitive risk factors, the empirical evidence for the causal relation of these factors to chronic depression is inconsistent, which might be largely due to an over reliance on correlational research designs (Hölzel, Härter, Reese, \& Kriston, 2011). Four risk factors to chronic depression that have received the most consistent empirical support in the literature are (1) early adversity, (2) personality (pathology), (3) cognitive factors, and (4) interpersonal factors. In order to describe the interplay between these factors we developed a cognitive schema model of chronic depression (Figure 1). In this model the effect of distal risk factors (early adversity) on chronic depression is mediated by proximate risk factors (early maladaptive schemas \& dysfunctional attitudes). Proximate risk factors are triggered by current life events, such as the experience of loss or failure, and they are maintained by avoidance related coping strategies (schema avoidance) and interpersonal behaviors that lead to schema maintenance. Interpersonal behaviors that are related to the avoidance of social situations or the avoidance of conflict (nonassertiveness) in turn contribute to the maintenance of depression through a lack of positive social reinforcement. In this model, distal risk-factors can best be understood as characteristics that increase the vulnerability of a person to experience proximate riskfactors. For example, experiencing childhood abuse might increase the vulnerability of developing a strong abandonment/instability schema. 


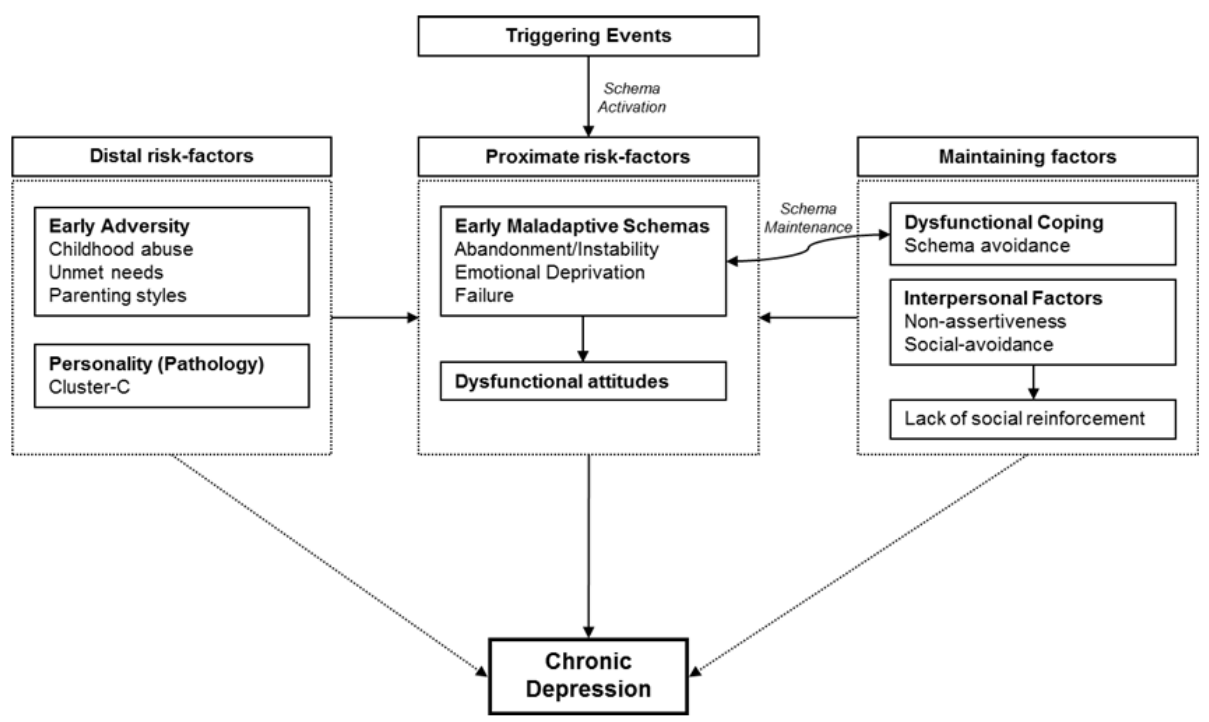

Figure 1. Cognitive schema model of chronic depression

Proximate risk-factors can best be understood as characteristics that more directly contribute to chronic depression in the context of recent triggering events. According to the model in Figure 1, a recent triggering event (for example the recent experience of abandonment or loss) can activate underlying EMS (for example abandonment/instability) which in turn directly contributes to chronic depression. Although three different types of dysfunctional coping mechanisms have been mentioned earlier, only schema avoidance was included in the model because this coping strategy is hypothesized to be the predominantly used coping strategy in clients with chronic depression. In the following section we review the empirical evidence for each of the four risk factors to chronic depression and describe how they can be targeted in ST.

\section{Early Adversity}

Early adversity is an umbrella term for childhood experiences such as, sexual, physical, and emotional abuse, physical and emotional neglect and parental behaviors such as rejection, indifference, or overcontrol. Compared to non-chronic forms of depression, chronic depression is more often characterized by early onset (Hölzel, et al., 2011) and hence the relation of developmental factors, especially early adversity, and chronic depression has been emphasized. Previous studies relating early adversity to chronic depression found that, compared to non-chronic forms of depression, clients with chronic depression more often report childhood adverse events (Brown, Craig, Harris, Handley, \& Harvey, 2007; Brown \& Moran, 1994; Hayden \& Klein, 2001; Lizardi \& Klein, 2000) and the experience of such events has been shown to be related to lower remission rates following a 12-week protocol of ADM (Klein et al., 2009). One large 
cohort study found that especial emotional abuse was related to risk for depression and that risk for depression (lifetime and current) increased gradually with the number of early adverse events in childhood (Chapman et al., 2004).

Childhood adversity usually precedes the onset of depression by several years and the relation between childhood adverse events and chronic depression is likely mediated by proximal risk factors (Klein \& Santiago, 2003). According to cognitive models of depression the way in which children cognitively organize adverse childhood experiences in cognitive schemas determines whether the child will develop depression at a later age (Ingram, 2003). In terms of the schema model, EMS are proximate risk factors that might mediate the relation between childhood adversity and psychopathology (Young, et al., 2003). In line with this, there is evidence that the relation between childhood adversity and depressive symptoms is mediated by specific sets of EMS in non-clinical (Harris \& Curtin, 2002) and in clinical samples (Cukor \& McGinn, 2006; Lumley \& Harkness, 2007; McGinn, Cukor, \& Sanderson, 2005). It has also been shown that the relation between childhood adversity and depression is mediated by a depressotypic schema organization in young adults (Lumley \& Harkness, 2009), a finding that has led to the conclusion that EMS in depression should be a focus of treatment in clients with a history of early adversity.

Childhood adversity in the treatment of chronic depression is associated with decreased response to ADM (Hayden \& Klein, 2001; Kaplan \& Klinetob, 2000) and higher response rates to psychotherapy (Nemeroff et al., 2003). Chronically depressed clients with a history of childhood adversity might therefore benefit more from forms of psychotherapy that specifically target childhood adversity. ST places special emphasis on childhood adversity. According to the schema model such experiences are the origin of EMS. In schema therapy experiential strategies, like imagery of traumatic events, are used to help the clients re-experience traumatic events in a safe therapeutic context, thereby decreasing the impact of the clients EMS.

\section{Cognitive Factors}

Dysfunctional cognitions in depression are usually conceptualized in terms of a hierarchical model of generality with automatic thoughts at the most superficial level, dysfunctional attitudes at an intermediate level, and cognitive schemas (or core beliefs) at the deepest level (Clark \& Beck, 1999; Segal, 1988). Most studies on cognitive factors in depression have focused on negative cognitions at the level of automatic thoughts or dysfunctional attitudes in relation to the onset and recurrences of depressive episodes and found that negative cognitive styles were related to increased likelihood of depression onset and recurrences (e.g., Alloy \& Abramson, 1999; Alloy et al., 2006; Burcusa \& Iacono, 2007; Lewinsohn, Joiner, \& Rohde, 2001; Mongrain \& Blackburn, 2005).

One previous study that has related cognitive factors to the course of depression focused on core beliefs at deeper levels of processing and found that avoidant and paranoid beliefs, as assessed with the Personality Beliefs Questionnaire (Beck \& Beck, 
1991), were related to poor outcome in cognitive therapy for depression (Kuyken, Kurzer, DeRubeis, Beck, \& Brown, 2001). In one prospective study, it has been shown that initially non-depressed individuals with high levels of negative cognitive styles and high levels of dysfunctional attitudes were more likely to experience a chronic course of depression at a 2.5 year follow-up assessment, compared to individuals with low levels of negative cognitive styles and dysfunctional attitudes (Iacoviello, Alloy, Abramson, Whitehouse, \& Hogan, 2006). In line with this, it has been shown in a prospective design that individuals with highly dysfunctional cognitions were lees likely to improve from a major depressive episode (Dent \& Teasdale, 1988; Lewinsohn, Steinmetz, Larson, \& Franklin, 1981).

Studies assessing dysfunctional cognitive processing at the schema level in chronically depressed clients are rare. In one study it was found that clients with chronic depression exhibit higher levels of EMS compared to non-chronically depressed clients, even after controlling for depressive symptom severity and personality disorders (Riso et al., 2003). Moreover, it has been shown that depressed clients are characterized by specific EMS (failure, emotional deprivation and abandonment/instability) and that these schemas remain relatively stable following outpatient treatment for depression (Renner, Lobbestael, Peeters, Arntz, \& Huibers, 2012). These findings suggest that current outpatient treatments for depression leave the underlying vulnerability to depression, in terms of EMS, largely untouched and that depressed clients with high levels of EMS might benefit from ST (Renner, Lobbestael, et al., 2012) .

ST builds on CT and hence draws from cognitive techniques that are also applied in CT for depression to decrease negative thinking patterns. The ultimate goal of ST is to decrease schematic processing that is dominated by EMS (Young, et al., 2003). Using cognitive techniques, clients learn to gather evidence that is disconfirming their schemas and by doing so to understand, on a rational level, that their schema is false. Moreover experiential techniques are used to help the client to feel on an emotional level that their schema is false. These different levels of schematic processing refer to the idea that there are different levels of meaning corresponding to rational propositional meanings (knowing cognitively) and holistic implicational meanings (knowing emotionally; Teasdale, 1999). CT and ST both target maladaptive schemas at the deepest (schema) level of cognition but ST also draws on experiential techniques to do so. By drawing from both cognitive and experiential techniques, ST appears to be better equipped to enable change in dysfunctional schematic processing at the schema level.

\section{Personality Pathology}

Several studies have demonstrated increased rates of axis-II psychopathology in clients with chronic forms of depression compared to clients with episodic forms of depression (Anderson et al., 1996; Fava et al., 1996; Hayden \& Klein, 2001; Holmstrand, Engström, \& Träskman-Bendz, 2008; Pepper et al., 1995). A number of theoretical models have been proposed to account for the relation between personality (disorders) and depression (Bagby, Quilty, \& Ryder, 2008; Farmer \& Nelson-Gray, 1990) but the large 
majority of studies relating personality disorders to chronic depression is based on cross-sectional data and hence the causal direction of this relation remains unclear.

Most studies on the relation between PD and depression focus on the impact of PD on treatment outcome. Recent meta-analyses suggest that comborbid PD doubles the risk of poor outcome in MDD treatment and hence it has been stressed that PD comorbidity should be addressed in depression treatment (Newton-Howes, Tyrer, \& Johnson, 2006). Relatively fewer studies investigated the relation between PD and treatment outcome in chronically depressed clients. One study found that comorbid PD had no negative impact on treatment outcome in chronic depression (Maddux et al., 2009) whereas in another study it was found that comorbid PD, especially BPD, predicted lower remission rates in depressed clients (Skodol et al., 2011). A number of possible explanations might account for these differential findings. The study of Maddux and colleagues (2009) drew data from a randomized controlled trial whereas the study of Skodol and colleagues (2011) related PDs to the course of depression in a prospective epidemiological study. Whereas the former study excluded clients with severe BPD, the latter study found that especially BPD clients had lower remission rates of depression. Whether or not co-morbid PDs have a negative impact of treatment outcome in depression might therefore depend in part on the types of PDs that are studied.

Although it remains to be investigated which specific PDs have a negative impact on treatment outcome in chronic depression, the findings that PD comorbidity is common in chronic depression, and has a negative impact on treatment outcome in episodic forms of depression suggests that PD should be addressed in the treatment of chronic depression.

ST was specifically developed for clients with chronic characterological problems who often present with axis-II disorders (Young, et al., 2003). With a special emphasis on characterological dysfunctional life patterns, ST has been shown to be an effective treatment for clients with BPD (Giesen-Bloo, et al., 2006) and clients with Cluster-C, paranoid, histrionic and narcissistic personality disorders (Bamelis, et al., 2012). Both BPD and Cluster-C personality disorders frequently co-occur with chronic depression (Farmer \& Nelson-Gray, 1990; Rothschild \& Zimmerman, 2002; Russell et al., 2003) and have been shown to be related to shorter survival times to depressive relapse (Ilardi, Craighead, \& Evans, 1997). Treating the underlying vulnerability to chronic depression likely also involves treating the underlying personality (features) that might drive depression and depressive relapse.

\section{Interpersonal Factors}

Depressed clients are characterized by an interpersonal style that can best be described as a blend between social-avoidance and non-assertiveness (Barrett \& Barber, 2007; Renner et al., 2012). This depressogenic interpersonal style remains stable after cognitive therapy (CT) for depression (Renner, Jarrett, et al., 2012). Studies relating interpersonal problems to depression chronicity are rare. In a cross-sectional design, it has been shown that chronically depressed inpatients report higher scores on the social 
avoidance subscale of the inventory of interpersonal problems, compared to nonchronically depressed inpatients (Ley et al., 2011). In a prospective study, it has been shown that interpersonal problems were related to depression chronicity at a follow up assessment and that interpersonal problems partially mediated the relation between childhood adversity and chronic depression (Brown \& Moran, 1994). Joiner (2000) developed a model of chronic depression with a strong emphasis on interpersonal factors. According to this model, chronic depression is characterized by increased negative-feedback seeking, reassurance seeking, and conflict avoidance, and accordingly these interpersonal difficulties should be addressed in the treatment of chronic depression (Joiner, 2000). Finally, it has been shown that the relation between a socially-avoidant interpersonal style and depression is likely mediated by reduced social positive reinforcement (Carvalho \& Hopko, 2011; Ferster, 1973).

One form of psychotherapy that specifically focusses on interpersonal stressors is Interpersonal Therapy (IPT) for depression (Klerman, Weissman, Rounsaville, \& Chevron, 1984). IPT focuses on a number of interpersonal sources of distress including interpersonal problems, role transitions, grief or interpersonal deficits that are assumed to give rise to depressed mood. IPT for depression is a time limited structured form of psychotherapy that focuses on a current acute episode of depression (Klerman, et al., 1984). For example if a client suffers from symptoms of depression following a job promotion, role transition might be the focus of treatment. In contrast to the focus on recent interpersonal sources of distress in_IPT, in the current theoretical framework interpersonal problems are conceptualized as rigid behavioral patterns in interpersonal situations. ST aims to break patterns such as a general avoidance of conflict (nonassertiveness) or avoidance of social-situations by drawing from behavioral pattern breaking techniques. Pattern breaking is the last step in schema therapy and crucial to avoid relapse and recurrences (Young, et al., 2003). In order to achieve pattern breaking, the client and therapist work out a list of targets and then start with the most problematic behaviors. Flash cards and homework assignments are used to practice healthy behaviors outside the therapy session whereas imagery and role-playing techniques of problematic interpersonal behaviors might be used during the therapy session. Additionally, ST draws from classical behavioral techniques such as assertiveness training in order to target interpersonal problems (Young, et al., 2003).

It should be noted that these techniques to target interpersonal problems are not unique to ST. These techniques might also be used in other therapy models such as CT, IPT, or in assertiveness training. The strength of ST in using these techniques is that the client and the therapist can identify and deal with EMS and schema modes that prevent the client from developing a more healthy interpersonal style. For example, clients with chronic depression might have a strong side to themselves that protects them from feelings of vulnerability (detached protector mode). In order to break through interpersonal patterns of behavior such as avoidance of conflict it might be necessary to bypass these protective sides to the self. 


\section{Similarities and differences with other treatment approaches}

Several of the ST techniques that can be used to address the risk-factors of chronic depression are not unique to ST but are also common to other forms of psychotherapy like CT or emotion focused therapy (EFT). It is therefore important to distinguish these approaches from ST and to highlight the distinctive features of ST that might make this approach superior to current approaches in the treatment of chronic depression. In this section we highlight some of the features that distinguish ST from CT because these two approaches are probably the most similar. For a detailed comparison of ST with other psychotherapy models, including emotion focused therapy, see (Young, et al., 2003).

Schema therapy is based on CT and therefore these two treatment approaches bear great similarity (Young, et al., 2003). For example, both treatments target maladaptive schemas by means of empirical verification (collaborative empiricism) to change these cognitive structures. In addition to these pure cognitive techniques ST also places great emphasis on experiential techniques to identify specific schemas and to change schemas (Young, et al., 2003). Previous research has shown that experiential techniques such as imagery rescripting have positive effects on depression in patients with primarily recurrent and chronic depression (Brewin et al., 2009). Moreover, in ST the therapeutic relationship is used as an active ingredient to change schemas whereas the therapeutic relationship in CT is seen as a basis for cognitive techniques to be effective rather than a therapeutic technique in itself (Young, et al., 2003). In this regard schema therapists are equipped with a richer toolbox of therapeutic techniques that can be used to target schemas from different perspectives. Another important difference between these two treatment approaches is that ST places a greater emphasis on early childhood experiences whereas traditional CT places more emphasis on the "here and now" (Beck, Rush, Shaw, \& Emery, 1979). Accordingly, in ST the techniques described above are also used to explore the origin of schemas in the past and to link past events to current problems.

As was argued, chronic depression is often rooted in adverse early childhood experiences that give rise to rigid, trait-like early maladaptive schemas and co-occurring personality pathology. It is therefore important that treatment of chronic depression takes into account the developmental aspects of this disorder and targets the various risk-factors via multiple channels. Traditional CT is primarily focused on the "here and now" and has a stronger emphasis on purely cognitive and behavioral techniques. The strength of ST as a treatment for chronic depression is that it draws from various therapeutic techniques, not only cognitive behavioral, and has a strong focus on the developmental context in which schemas develop.

\section{Treatment protocol}

The treatment protocol of ST for chronic depression is based on the basic protocol of ST developed by Young et al. (2003). The treatment protocol of ST for chronic depression can be divided into three phases: (1) Exploration, (2) Change, (3) Relapse prevention. In 
the first two phases sessions should be scheduled weekly, whereas in the last phase the frequency of sessions should be decreased to give the client more autonomy and responsibility.

\section{Phase I - Exploration (session 1-10)}

There are three main goals in the first phase of treatment. First, clients need to understand the concept of schemas and identify their predominant schemas. Second, relations between the clients' predominant schemas, their current problems and their history are established. Third, the therapist helps the client to experience the feelings that are associated with their schemas by drawing from experiential techniques. For example, imagery of childhood events can be used to identify predominant underlying schemas. In these exercises, the client is instructed to recall a distressing event from their childhood while the therapist observes the affective reactions of the client. Client and therapist then move from more general images to more specific images that include significant others (for example the parents) in order to cover the whole range of early maladaptive schemas experienced by the client (Young, et al., 2003). In contrast to the imagery techniques that are used in the change phase, imagery in the exploration phase does not include rescripting of the images.

Phase II - Change

In the change phase different therapeutic techniques and interventions are used to work on change in EMS, emotional experiences and dysfunctional behaviors. Four types of interventions are used in the change phase: (1) cognitive techniques, (2) experiential techniques, (3) the therapeutic alliance, (4) behavioral techniques.

\section{Cognitive techniques}

Cognitive techniques are used to help the client to develop a more rational and objective way of thinking. The goal is to develop a more realistic view of the self and the world. The specific techniques that are used here are largely drawn from techniques that are used in cognitive therapy for depression (Beck, et al., 1979).

\section{Experiential techniques}

Experiential techniques are used to work on emotional experiences within the session. Experiential techniques that can be used in ST for chronic depression are imagery (rescripting) and chair dialogues. Imagery (rescripting) of current problematic situations and past experiences is used to teach the client to take different positions in, for example, unsafe, negative childhood experiences. By using these techniques the client learns to deal with experiences of incapacity and despair, to express basic needs to others, to develop healthy defense mechanisms, to create a sense of safety in problematic situations, and to adequately express negative emotions such as anger. The therapist takes a limited reparenting stance in this phase by acting as a good parent to 
meet the needs that the clients' inner child has missed in the past within the appropriate boundaries of therapeutic context. The aim is to provide the client with the needs that were not met in the past in order to make the way for further development. For example, the therapist provides the client with solace and support while at the same time provides clear boundaries.

Another important experiential technique that can be used in ST for chronic depression is chair dialogue. Chair dialogues have their roots in Gestalt Therapy and are also used in other therapy models for depression such as emotion focused therapy (Greenberg \& Watson, 2006). In chair dialogues, every chair represents a different side of the self of the client. In schema therapy terms these different sides of clients are referred to as schema modes. For example, depressed clients might have a strong overly demanding side (demanding parent mode) to themselves while at the same time the healthy part of the self (healthy adult mode) might be weak. Although chair dialogues are usually conducted between two sides of the client it is also possible that more than two sides (modes) are involved (multiple chair dialogue). During chair dialogues the client is trained to set boundaries to the impact of his/her own punishing and overly demanding sides while at the same time training to further develop and strengthen the own healthy side (health adult mode) that can care for the inner vulnerable child side (vulnerable child mode) of the client. During chair work the therapist guides the different sides (modes) of the client through the dialogues.

\section{Therapeutic alliance}

The therapeutic alliance is an important instrument in ST. The therapist confronts the client with the client's in-session behaviors that are driven by the client's underlying EMSs by means of empathic confrontation. For example, a client who constantly inquires about the wellbeing of the therapist might repeat patterns of earlier learned parentification behaviors towards the parents. By means of empathic confrontation and exploration of these behaviors the client can learn to take a different stance. As was the case with the experiential techniques, the therapist takes the position of a good parent (limited reparenting) by exploring the needs of the client that were not met during childhood. The therapist tries to provide the client with these needs on the one hand while on the other hand provide the clients with the means to independently care for his/her basic needs.

\section{Behavioral techniques}

Important behavioral techniques that can be used in ST for chronic depression include role-playing, homework assignments, behavioral experiments and assertiveness training. By using these techniques, the client learns to put the cognitive and emotional insights learned in therapy into action. For example, a student who is afraid of rejection and negative feedback by his/her supervisor can be encouraged by the therapist to discuss these issues with the supervisor and making his/her needs known by asking for objective feedback. 


\section{Phase III - Relapse prevention}

During the last phase of therapy the client and therapist create a plan on how to prevent relapse. This includes an analysis of situations and events that might have the potential to trigger relapse and a plan for how to cope with these situations. During this last phase of therapy, sessions are scheduled with lower frequencies. The client receives more control over the sessions and the healthy adult side of the client that was developed during the previous phase is further coached and supported by the therapist in order to give way to the development of more confidence and autonomy.

\section{Concluding remarks}

ST has become a promising treatment approach for clients with difficult to treat personality disorders. While there is a need for further research on the effectiveness of ST for axis-II disorders, it is also important to consider new adaptations of ST to chronic axis-I disorders. We have argued that ST is a potentially effective treatment for chronic depression as it targets important underlying risk factors to chronic depression. It should be noted, however, that there is no empirical evidence to date that ST is an effective treatment for clients with chronic depression. In order to test whether ST is a potentially effective treatment for chronic depression, we are currently conducting a single-case series of ST for chronic depression in the Netherlands (ClinicalTrials.gov Identifier: omitted for blinded review purpose). In this study 20 clients with chronic MDD receive up to 75 individual sessions of ST. In addition to standardized assessment instruments for depressive symptom severity and potential mediators of treatment outcome we are using an interview technique to assess schemas and core beliefs on an idiosyncratic level. Moreover, we are using functional Magnetic Resonance Imaging (fMRI) to study the underlying neural mechanisms of change in ST. The results of this study will provide the first empirical evidence on the effectiveness of ST as a novel treatment approach to chronic MDD and first insights into the underlying (neurobiological) working mechanisms of ST for chronic depression.

Besides the need to test whether ST is a potentially effective treatment for chronic depression it is also important to further study basic concepts of schema theory in (chronically) depressed clients. There is now some evidence that depressed clients are characterized by a specific set of EMS, yet, research into schema modes in depression is absent. The working model of chronic depression that we presented is based on underlying factors that have received the most consistent empirical support to date. As new evidence emerges, the model can be adapted to describe the role of specific schema modes in chronic depression.

In conclusion, ST is a promising new treatment approach for clients with severe chronic depressions because it directly targets the underlying vulnerability to chronic depression, as described in our working model, and is therefore likely to lead to positive and enduring outcomes. Future research on the effectiveness of ST for chronic 
depression needs to be conducted comparing ST to other treatment options for chronic depression in larger samples in the context of randomized clinical trials. 


\section{References}

Alloy, L. B., \& Abramson, L. Y. (1999). The TempleWisconsin Cognitive Vulnerability to Depression Project: Conceptual Background, Design, and Methods. Journal of Cognitive Psychotherapy, 13, 227-262.

Alloy, L. B., Abramson, L. Y., Whitehouse, W. G., Hogan, M. E., Panzarella, C., \& Rose, D. T. (2006). Prospective incidence of first onsets and recurrences of depression in individuals at high and low cognitive risk for depression. Journal of Abnormal Psychology, 115(1), 145-156.

Anderson, R. L., Klein, D. N., Riso, L. P., Ouimette, P. C., Lizardi, H., \& Schwartz, J. E. (1996). The subaffectivecharacter spectrum subtyping distinction in primary early-onset dysthymia : a clinical and family study (Vol. 38). Oxford, ROYAUME-UNI: Elsevier.

Arnow, B. A., \& Constantino, M. J. (2003). Effectiveness of psychotherapy and combination treatment for chronic depression. Journal of Clinical Psychology, 59(8), 893-905.

Bagby, R., Quilty, L. C., \& Ryder, A. (2008). Personality and Depression. The Canadian Journal of Psychiatry, 53(1), 14-25.

Bamelis, L. L. M., Evers, S. M. A. A., Spinhoven, P., \& Arntz, A. (2014). Results of a multicentered randomized controlled trial on the clinical effectiveness of schema therapy for personality disorders. Am $J$ Psychiatry; 171:305-322

Barrett, M. S., \& Barber, J. P. (2007). Interpersonal profiles in major depressive disorder. Journal of Clinical Psychology, 63(3), 247-266.

Beck, A. T., \& Beck, J. S. (1991). The Personality Belief Questionnaire. Unpublished assessment instrument. Bala Cynwyd, PA: : The Beck Institute for Cognitive Therapy and Research.

Beck, A. T., Rush, A. J., Shaw, B. F., \& Emery, G. (1979). Cognitive therapy of depression. New York: Guilford.

Brewin, C. R., Wheatley, J., Patel, T., Fearon, P., Hackmann, A., Wells, A., . . Myers, S. (2009). Imagery rescripting as a brief stand-alone treatment for depressed patients with intrusive memories. Behaviour Research and Therapy, 47(7), 569-576. doi: 10.1016/j.brat.2009.03.008

Brown, G. W., Craig, T. K. J., Harris, T. O., Handley, R. V., \& Harvey, A. L. (2007). Development of a retrospective interview measure of parental maltreatment using the Childhood Experience of Care and Abuse (CECA) instrument -- A life-course study of adult chronic depression -- 1. Journal of Affective Disorders, 103(1-3), 205-215.

Brown, G. W., \& Moran, P. (1994). Clinical and psychosocial origins of chronic depressive episodes: I. A community survey. British Journal of Psychiatry, 165(4), 447-456.

Browne, G., Steiner, M., Roberts, J., Gafni, A., Byrne, C., Dunn, E., . . Wallik, D. (2002). Sertraline and/or interpersonal psychotherapy for patients with dysthymic disorder in primary care: 6-month comparison with longitudinal 2-year follow-up of effectiveness and costs. Journal of Affective Disorders, 68, 317 - 330.

Burcusa, S. L., \& Iacono, W. G. (2007). Risk for recurrence in depression. Clinical Psychology Review, 27(8), 959-985.

Carvalho, J. P., \& Hopko, D. R. (2011). Behavioral theory of depression: Reinforcement as a mediating variable between avoidance and depression. Journal of Behavior Therapy and Experimental Psychiatry, 42(2), 154-162.

Castonguay, L. G., Goldfried, M. R., Wiser, S., Raue, P. J., \& Hayes, A. M. (1996). Predicting the effect of cognitive therapy for depression: A study of unique and common factors. Journal of Consulting and Clinical Psychology, 64(3), 497-504. doi: 10.1037/0022-006x.64.3.497

Chapman, D. P., Whitfield, C. L., Felitti, V. J., Dube, S. R., Edwards, V. J., \& Anda, R. F. (2004). Adverse childhood experiences and the risk of depressive disorders in adulthood. Journal of Affective Disorders, 82(2), 217-225. doi: 10.1016/j.jad.2003.12.013

Clark, D. M., \& Beck, A. T. (1999). Scientific foundations of cognitive theory and therapy for depression. New York: Wiley.

Cuijpers, P., van Straten, A., Schuurmans, J., van Oppen, P., Hollon, S. D., \& Andersson, G. (2010). Psychotherapy for chronic major depression and dysthymia: A meta-analysis. Clinical Psychology Review, 30(1), 51-62. 
Cukor, D., \& McGinn, L. K. (2006). History of Child Abuse and Severity of Adult Depression: The Mediating Role of Cognitive Schema. Journal of Child Sexual Abuse, 15(3), 19 - 34.

Dent, J., \& Teasdale, J. D. (1988). Negative cognition and the persistence of depression. Journal of Abnormal Psychology, 97(1), 29-34.

Ellison, J. A., Greenberg, L. S., Goldman, R. N., \& Angus, L. (2009). Maintenance of gains following experiential therapies for depression. Journal of Consulting and Clinical Psychology, 77(1), 103-112. doi: $10.1037 / \mathrm{a} 0014653$

Farmer, R., \& Nelson-Gray, R. O. (1990). Personality disorders and depression: Hypothetical relations, empirical findings, and methodological considerations. Clinical Psychology Review, 10(4), 453-476.

Farrell, J. M., Shaw, I. A., \& Webber, M. A. (2009). A schema-focused approach to group psychotherapy for outpatients with borderline personality disorder: A randomized controlled trial. Journal of Behavior Therapy and Experimental Psychiatry, 40(2), 317-328.

Fava, M., Alpert, J., Borus, J., Nierenberg, A., Pava, J., \& Rosenbaum, J. (1996). Patterns of personality disorder comorbidity in early-onset versus late- onset major depression. American Journal of Psychiatry, 153(10), 1308-1312.

Ferster, C. B. (1973). A functional analysis of depression. American Psychologist, 28(10), 857-870.

Gelenberg, A. J., Trivedi, M. H., Rush, A. J., Thase, M. E., Howland, R., Klein, D. N., . . Keller, M. B. (2003). Randomized, Placebo-Controlled Trial of Nefazodone Maintenance Treatment in Preventing Recurrence in Chronic Depression. Biological Psychiatry, 54(8), 806-817.

Giesen-Bloo, J., van Dyck, R., Spinhoven, P., van Tilburg, W., Dirksen, C., van Asselt, T., ... Arntz, A. (2006). Outpatient Psychotherapy for Borderline Personality Disorder: Randomized Trial of SchemaFocused Therapy vs Transference-Focused Psychotherapy. Archives of General Psychiatry, 63(6), 649-658.

Greenberg, L. S., \& Watson, J. C. (2006). Emotion-Focused Therapy for Depression. Washington: American Psychological Association.

Harris, A. E., \& Curtin, L. (2002). Parental perceptions, early maladaptive schemas, and depressive symptoms in young adults. Cognitive Therapy and Research, 26(3), 405-416.

Hayden, E. P., \& Klein, D. N. (2001). Outcome of Dysthymic Disorder at 5-Year Follow-Up: The Effect of Familial Psychopathology, Early Adversity, Personality, Comorbidity, and Chronic Stress. American Journal of Psychiatry, 158(11), 1864-1870. doi: 10.1176/appi.ajp.158.11.1864

Holmstrand, C., Engström, G., \& Träskman-Bendz, L. (2008). Disentangling dysthymia from major depressive disorder in suicide attempters' suicidality, comorbidity and symptomatology. Nordic Journal of Psychiatry, 62(1), 25-31. doi: doi:10.1080/08039480801960164

Hölzel, L., Härter, M., Reese, C., \& Kriston, L. (2011). Risk factors for chronic depression -- A systematic review. Journal of Affective Disorders, 129(1-3), 1-13.

Howland, R. H. (1993). Chronic depression. Hospital \& Community Psychiatry, 44(7), 633-639.

Iacoviello, B. M., Alloy, L. B., Abramson, L. Y., Whitehouse, W. G., \& Hogan, M. E. (2006). The course of depression in individuals at high and low cognitive risk for depression: A prospective study. Journal of Affective Disorders, 93(1-3), 61-69.

Ilardi, S. S., Craighead, W. E., \& Evans, D. D. (1997). Modeling relapse in unipolar depression: The effects of dysfunctional cognitions and personality disorders. Journal of Consulting and Clinical Psychology, 65(3), 381-391.

Ingram, R. E. (2003). Origins of Cognitive Vulnerability to Depression. Cognitive Therapy and Research, 27(1), 77-88. doi: 10.1023/a:1022590730752

James, I. A., Southam, L., \& Blackburn, I. M. (2004). Schemas Revisited. Clinical Psychology \& Psychotherapy, 11(6), 369-377.

Joiner, T. E. (2000). Depression's Vicious Scree: Self-Propagating and Erosive Processes in Depression Chronicity. Clinical Psychology: Science and Practice, 7(2), 203-218.

Kaplan, M. J., \& Klinetob, N. A. (2000). Childhood Emotional Trauma and Chronic Posttraumatic Stress Disorder in Adult Outpatients with Treatment-Resistant Depression. The Journal of nervous and mental disease, 188(9), 596-601. 
Keller, M. B., Hanks, D. L., Kocsis, J. H., \& Klein, D. N. (1995). Course and natural history of chronic depression Diagnosis and treatment of chronic depression. (pp. 58-72). New York, NY US: Guilford Press.

Keller, M. B., McCullough, J. P., Klein, D. N., Arnow, B., Dunner, D. L., Gelenberg, A. J., . . Zajecka, J. (2000). A comparison of nefazodone, the cognitive behavioral-analysis system of psychotherapy, and their combination for the treatment of chronic depression. The New England Journal of Medicine, 342(20), 1462-1470.

Klein, D. N. (2010). Chronic Depression. Current Directions in Psychological Science, 19(2), 96-100. doi: $10.1177 / 0963721410366007$

Klein, D. N., Arnow, B. A., Barkin, J. L., Dowling, F., Kocsis, J. H., Leon, A. C., . . Wisniewski, S. R. (2009). Early adversity in chronic depression: clinical correlates and response to pharmacotherapy. Depression \& Anxiety (1091-4269), 26(8), 701-710.

Klein, D. N., \& Santiago, N. (2003). Dysthymia and chronic depression: Introduction, classification, risk factors, and course. Journal of Clinical Psychology, 59(8), 807-816.

Klerman, G., Weissman, M., Rounsaville, B., \& Chevron, E. (1984). Interpersonal psychotherapy of depression. New York: Basic Books.

Kocsis, J. H. (2003). Pharmacotherapy for chronic depression. Journal of Clinical Psychology, 59(8), 885892.

Kocsis, J. H., Gelenberg, A. J., Rothbaum, B. O., Klein, D. N., Trivedi, M. H., Manber, R., . . for the REVAMP Investigators. (2009). Cognitive Behavioral Analysis System of Psychotherapy and Brief Supportive Psychotherapy for Augmentation of Antidepressant Nonresponse in Chronic Depression: The REVAMP Trial. Archives of General Psychiatry, 66(11), 1178-1188. doi: 10.1001/archgenpsychiatry.2009.144

Kuyken, W., Kurzer, N., DeRubeis, R. J., Beck, A. T., \& Brown, G. K. (2001). Response to cognitive therapy in depression: The role of maladaptive beliefs and personality disorders. Journal of Consulting and Clinical Psychology, 69(3), 560-566. doi: 10.1037/0022-006x.69.3.560

Lewinsohn, P. M., Joiner, T. E., Jr., \& Rohde, P. (2001). Evaluation of cognitive diathesis-stress models in predicting major depressive disorder in adolescents. Journal of Abnormal Psychology, 110(2), 203215. doi: 10.1037/0021-843x.110.2.203

Lewinsohn, P. M., Steinmetz, J. L., Larson, D. W., \& Franklin, J. (1981). Depression-related cognitions: Antecedent or consequence? Journal of Abnormal Psychology, 90(3), 213-219.

Ley, P., Helbig-Lang, S., Czilwik, S., Lang, T., Worlitz, A., BrÃ¹/4cher, K., \& Petermann, F. (2011). Phenomenological differences between acute and chronic forms of major depression in inpatients. Nordic Journal of Psychiatry, O(0), 1-8. doi: doi:10.3109/08039488.2011.552121

Lizardi, H., \& Klein, D. N. (2000). Parental psychopathology and reports of the childhood home environment in adults with early-onset dysthymic disorder. The Journal of nervous and mental disease, 188(2), 63-70.

Lumley, M. N., \& Harkness, K. L. (2007). Specificity in the relations among childhood adversity, early maladaptive schemas, and symptom profiles in adolescent depression. Cognitive Therapy and Research, 31(5), 639-657.

Lumley, M. N., \& Harkness, K. L. (2009). Childhood maltreatment and depressotypic cognitive organization. Cognitive Therapy and Research, 33(5), 511-522. doi: 10.1007/s10608-009-9257-7

Maddux, R. E., Riso, L. P., Klein, D. N., Markowitz, J. C., Rothbaum, B. O., Arnow, B. A., ... Thase, M. E. (2009). Select comorbid personality disorders and the treatment of chronic depression with nefazodone, targeted psychotherapy, or their combination. Journal of Affective Disorders, 117(3), 174-179.

Manber, R., Kraemer, H. C., Arnow, B. A., Trivedi, M. H., Rush, A. J., Thase, M. E., . . Keller, M. E. (2008). Faster remission of chronic depression with combined psychotherapy and medication than with each therapy alone. Journal of Consulting and Clinical Psychology, 76(3), 459-467.

McCullough, J. P., Jr. (2003). Treatment for chronic depression using cognitive behavioral analysis system of psychotherapy (CBASP). Journal of Clinical Psychology, 59(8), 833-846. 
McCullough, J. P., Jr., Klein, D. N., Borian, F. E., Howland, R. H., Riso, L. P., Keller, M. B., \& Banks, P. L. C. (2003). Group Comparisons of DSM-IV Subtypes of Chronic Depression: Validity of the Distinctions, Part 2. Journal of Abnormal Psychology, 112(4), 614-622.

McGinn, L. K., Cukor, D., \& Sanderson, W. C. (2005). The Relationship Between Parenting Style, Cognitive Style, and Anxiety and Depression: Does Increased Early Adversity Influence Symptom Severity Through the Mediating Role of Cognitive Style? Cognitive Therapy and Research, 29(2), 219-242.

Mongrain, M., \& Blackburn, S. (2005). Cognitive Vulnerability, Lifetime Risk, and the Recurrence of Major Depression in Graduate Students. Cognitive Therapy and Research, 29(6), 747-768. doi: 10.1007/s10608-005-4290-7

Nadort, M., Arntz, A., Smit, J. H., Giesen-Bloo, J., Eikelenboom, M., Spinhoven, P., . . van Dyck, R. (2009). Implementation of outpatient schema therapy for borderline personality disorder with versus without crisis support by the therapist outside office hours: A randomized trial. Behaviour Research and Therapy, 47(11), 961-973.

Nemeroff, C. B., Heim, C. M., Thase, M. E., Klein, D. N., Rush, A. J., Schatzberg, A. F., . . Keller, M. B. (2003). Differential responses to psychotherapy versus pharmacotherapy in patients with chronic forms of major depression and childhood trauma. Proceedings of the National Academy of Sciences of the United States of America, 100(24), 14293-14296. doi: 10.1073/pnas.2336126100

Newton-Howes, G., Tyrer, P., \& Johnson, T. (2006). Personality disorder and the outcome of depression: Meta-analysis of published studies. British Journal of Psychiatry, 188(1), 13-20.

Nordahl, H. M., Holthe, H., \& Haugum, J. A. (2005). Early Maladaptive Schemas in Patients with or without Personality Disorders: Does Schema Modification Predict Symptomatic Relief? Clinical Psychology \& Psychotherapy, 12(2), 142-149.

Pampallona, S., Bollini, P., Tibaldi, G., Kupelnick, B., \& Munizza, C. (2004). Combined Pharmacotherapy and Psychological Treatment for Depression: A Systematic Review. Archives of General Psychiatry, 61(7), 714-719. doi: 10.1001/archpsyc.61.7.714

Pepper, C., Klein, D., Anderson, R., Riso, L., Ouimette, P., \& Lizardi, H. (1995). DSM-III-R axis II comorbidity in dysthymia and major depression. American Journal of Psychiatry, 152(2), 239-247.

Renner, F., Jarrett, R. B., Vittengl, J. R., Barrett, M. S., Clark, L. A., \& Thase, M. E. (2012). Interpersonal problems as predictors of therapeutic alliance and symptom improvement in cognitive therapy for depression. Journal of Affective Disorders, 138(3), 458-467.

Renner, F., Lobbestael, J., Peeters, F., Arntz, A., \& Huibers, M. (2012). Early maladaptive schemas in depressed patients: Stability and relation with depressive symptoms over the course of treatment. Journal of Affective Disorders, 136(3), 581-590.

Riso, L. P., du Toit, P. L., Blandino, J. A., Penna, S., Dacey, S., Duin, J. S., . . Ulmer, C. S. (2003). Cognitive aspects of chronic depression. Journal of Abnormal Psychology, 112(1), 72-80.

Riso, L. P., \& Newman, C. F. (2003). Cognitive therapy for chronic depression. Journal of Clinical Psychology, 59(8), 817-831.

Rothschild, L., \& Zimmerman, M. (2002). Personality Disorders and the Duration of Depressive Episode: A Retrospective Study. Journal of Personality Disorders, 16(4), 293-303. doi: 10.1521/pedi.16.4.293.24129

Russell, J. M., Kornstein, S. G., Shea, M. T., McCullough, J. P., Harrison, W. M., Hirschfeld, R. M. A., \& Keller, M. B. (2003). Chronic depression and comorbid personality disorders: Response to sertraline versus imipramine. Journal of Clinical Psychiatry, 64(5), 554-561.

Schramm, E., Zobel, I., Dykierek, P., Kech, S., Brakemeier, E.-L., Külz, A., \& Berger, M. (2011). Cognitive behavioral analysis system of psychotherapy versus interpersonal psychotherapy for early-onset chronic depression: A randomized pilot study. Journal of Affective Disorders, 129(1-3), 109-116.

Segal, Z. V. (1988). Appraisal of the self-schema construct in cognitive models of depression. Psychological Bulletin, 103(2), 147-162.

Skodol, A. E., Grilo, C. M., Keyes, K. M., Geier, T., Grant, B. F., \& Hasin, D. S. (2011). Relationship of Personality Disorders to the Course of Major Depressive Disorder in a Nationally Representative Sample. American Journal of Psychiatry, 168(3), 257-264. doi: 10.1176/appi.ajp.2010.10050695 
Smit, F., Cuijpers, P., Oostenbrink, J., Batelaan, N., de Graaf, R., \& Beekman, A. (2006). Costs of Nine Common Mental Disorders: Implications for Curative and Preventive Psychiatry. Journal of Mental Health Policy and Economics, 9(4), 193-200.

Stulz, N., Thase, M. E., Klein, D. N., Manber, R., \& Crits-Christoph, P. (2010). Differential effects of treatments for chronic depression: A latent growth model reanalysis. Journal of Consulting and Clinical Psychology, 78(3), 409-419. doi: 10.1037/a0019267

Teasdale, J. D. (1999). Emotional processing, three modes of mind and the prevention of relapse in depression. Behaviour Research and Therapy, 37(Suppl 1), S53-S77. doi: 10.1016/s00057967(99)00050-9

Thase, M. E. (1999). When are Psychotherapy and Pharmacotherapy Combinations the Treatment of Choice for Major Depressive Disorder? Psychiatric Quarterly, 70(4), 333-346. doi: 10.1023/a:1022042316895

Thase, M. E., Greenhouse, J. B., Frank, E., Reynolds, C. F., III, Pilkonis, P. A., Hurley, K., .. Kupfer, D. J. (1997). Treatment of Major Depression With Psychotherapy or Psychotherapy-Pharmacotherapy Combinations. Archives of General Psychiatry, 54(11), 1009-1015. doi: 10.1001/archpsyc.1997.01830230043006

Torpey, D. C., \& Klein, D. N. (2008). Chronic depression: Update on classification and treatment. Current Psychiatry Reports, 10, 458-464.

Watson, J. C., \& Bedard, D. L. (2006). Clients' emotional processing in psychotherapy: A comparison between cognitive-behavioral and process-experiential therapies. Journal of Consulting and Clinical Psychology, 74(1), 152-159. doi: 10.1037/0022-006x.74.1.152

Watson, J. C., Gordon, L. B., Stermac, L., Kalogerakos, F., \& Steckley, P. (2003). Comparing the effectiveness of process-experiential with cognitive-behavioral psychotherapy in the treatment of depression. Journal of Consulting and Clinical Psychology, 71(4), 773-781.

Wells, K. B., Burnam, M. A., Rogers, W., \& Hays, R. (1992). The course of depression in adult outpatients: Results from the Medical Outcomes Study. Archives of General Psychiatry, 49(10), 788-794.

Young, J. E., Klosko, J., \& Weishaar, M. E. (2003). Schema therapy: A practitioner's guide. New York: Guilford Press. 


\section{CHAPTER 9 \\ Effects of schema therapy for chronic depression}

Submitted as:

Renner, F., Arntz, A., Peeters, F., Lobbestael, J., \& Huibers, M. (2014). Schema therapy for chronic depression: Results of a multiple single case series study. 


\begin{abstract}
The aim of this study was to test the effects of individual schema therapy (ST) on depressive symptom severity, recovery from depression, global symptomatic distress and improvements in overall functioning in patients with a DSM-IV diagnosis of chronic major depressive disorder (cMDD). Using a multiple-baseline single case series design, patients with cMDD $(N=25)$ first completed weekly assessments during a 6 to 24 weeks non-treatment baseline phase. After this baseline phase, patients entered a 12 week exploration phase during which symptoms and underlying schemas were explored and treatment goals were set; this phase also functioned as an attention control condition. Next, patients received up to 65 sessions of individual ST at a specialized secondary mental health care facility. During all three phases depressed symptoms and overall functioning was assessed every week, resulting in an average of 100 repeated weekly assessments per participant, whereas global symptom severity was assessed every four weeks. Of the overall sample five patients dropped out during the baseline period, none dropped out during exploration or treatment. Of the remaining 20 patients who started treatment, $40 \%$ responded and 35\% remitted. Diagnostic assessments at post-treatment were available from 12 patients. Of these 12 patients, 58\% did not meet the diagnostic criteria for a depressive disorder anymore and were therefore considered recovered. Mixed model regression analyses yielded significant time within intervention effects for all continuous outcomes: When compared to the baseline period, the intervention phase had a significant, large effect on depressive symptom severity (Cohen's $d$ between 0.91 and 1.30), moderate to large effects on global symptomatic distress (Cohen's $d$ between 0.64 and 0.86 ), and large effects on improvements in overall functioning (Cohen's $d$ between 0.93 and 0.96 ). These findings provide preliminary evidence that ST is an effective treatment for patients with cMDD.
\end{abstract}

Keywords: schema therapy; chronic depression; single case series; long-term psychotherapy 


\section{Introduction}

About one in five patients with depression develops a chronic course defined as the presence of depressive symptoms for two years or longer (Keller, Hanks, Kocsis, \& Klein, 1995). Compared to non-chronic depression, chronic forms of depression are associated with a lower quality of life (Wells, Burnam, Rogers, \& Hays, 1992), increased suicide attempts and hospitalizations (Arnow \& Constantino, 2003; Torpey \& Klein, 2008), greater service use and higher economic costs (Howland, 1993; Smit et al., 2006). Pharmacological and psychotherapeutic interventions for chronic depression are effective but meta analyses have shown that the effect sizes of psychotherapy are generally low ( $d=0.23$; Cuijpers, van Straten, et al., 2010) and about half of the patients with chronic forms of depression do not remit following treatment with antidepressant medication (for a review see: Kocsis, 2003). The most positive treatment results are obtained when psychotherapy and pharmacotherapy are combined, with effect sizes exceeding those of either treatment alone (Cuijpers, van Straten, et al., 2010; Spijker, van Straten, Bockting, Meeuwissen, \& van Balkom, 2013). Given the negative consequences of chronic depression and the relatively low effects of current treatments, there is a need to further explore new treatment options. The aim of the present study was to test the effects of up to 75 individual sessions of schema therapy (ST) for patients with chronic major depressive disorder (cMDD).

Compared to patients with non-chronic depression, patients with chronic depression more frequently report a history of childhood adversity (Brown, Craig, Harris, Handley, \& Harvey, 2007; Brown \& Moran, 1994; Hayden \& Klein, 2001; Lizardi \& Klein, 2000) and increased rates of personality disorders (Fava et al., 1996; Hayden \& Klein, 2001; Holmstrand, Engström, \& Träskman-Bendz, 2008; Pepper et al., 1995). Such developmental factors and personality (disorders) can be conceptualized as underlying vulnerability to cMDD, meaning that they might increase the risk for developing depression as well as contributing to the maintenance of depression and thereby to depression chronicity. We have argued previously, that current treatments for (chronic) depression might not adequately take these factors into account, thereby leaving the underlying vulnerability to chronic depression untouched (Renner, Arntz, Leeuw, \& Huibers, 2013). Given that chronic depression is often rooted in negative childhood events and personality pathology, current short-term, symptom focused treatments for chronic depression might fall short in addressing these factors sufficiently. Moreover, a recent meta-analysis has shown that the effects of psychotherapy for chronic depression have a dose-effect relationship with the number of treatment sessions (Cuijpers, van Straten, et al., 2010). Thus patients with chronic depression likely benefit more from long-term psychotherapy that focusses on present symptoms as well as on the underlying vulnerability.

ST is an integrative long-term psychotherapy for chronic and difficult to treat axis-I and axis-II disorders (Young, Klosko, \& Weishaar, 2003). In ST special emphasis is placed on early childhood experiences and the relation of these experiences with current problems. Empirical evidence suggests that ST is effective in treating adults with 
borderline personality disorder (Giesen-Bloo et al., 2006; Nadort et al., 2009; Nordahl \& Nysaeter, 2005), and Cluster-C, paranoid, histrionic and narcissistic personality disorders (Bamelis, Evers, Spinhoven, \& Arntz, 2013). We have previously argued that ST is also a potential effective treatment for patients with chronic depression (Renner et al., 2013). Moreover, we have shown that patients with depression are characterized by a distinct set of early maladaptive schemas and have argued that depressed patients might benefit from addressing these schemas in ST (Renner, Lobbestael, Peeters, Arntz, \& Huibers, 2012).

Most studies to date have focused on the effects of ST for personality disorders. In 2009 our group started a single-case series of ST for cMDD as a first attempt to test whether ST is a potentially effective treatment for patients with cMDD too (ClinicalTrials.gov Identifier: NCT01153867). Since then, a number of studies have emerged supporting the effects of ST (techniques) for (chronic) depression. Brewin et al. (2009) reported positive and large effects of imagery rescripting, a key-technique in ST, on depressive symptom severity as a stand-alone intervention for treating patients with chronic depression. Carter et al. (2013) compared ST with cognitive therapy (CT) in a randomized controlled trial (RCT) focusing on patients with MDD. On average, participants received 22.3 sessions of ST (Carter et al., 2013), a less intense treatment protocol than we have previously suggested for patients with chronic depression (Renner et al., 2013). At the end of the study no differences emerged between the two conditions on the main study outcomes (Carter et al., 2013). In the ST condition $50 \%$ of the patients remitted ( $40 \%$ in CT) and $56 \%$ of those randomized to ST recovered (50\% in CT) (Carter et al., 2013). Results did not differ for a subgroup of patients with chronic depression (67\%), suggesting that this relatively short ST protocol for depression was as effective as CT for patients with and without chronic depression (Carter et al., 2013). Another study tested the effects of ST in women with chronic depression (dysthymic disorder, double depression or cMDD), using a single-case series design (Malogiannis et al., 2014). Following an eight week baseline period, 12 patients with chronic depression received up to 60 sessions of individual ST. The authors found large effects of ST on depressive symptom improvements and approximately $60 \%$ of patients remitted during treatment (Malogiannis et al., 2014).

Although findings from these studies are promising, there is a need to further test the effects of ST for patients with cMDD with an appropriate amount of sessions. The study by Carter et al. (2013) primarily focused on patients with non-chronic depression and had a limited number of treatment sessions which might not be sufficient for patients with cMDD. The study by Malogiannis et al. (2014) did not include multiple baselines in the case series making it more difficult to attribute effects to treatment rather than passing of time. Moreover, Malogiannis et al. (2014) did only include women with chronic depression thereby limiting the generalizability of findings. The current study advances previous studies by testing the effects of up to 75 individual sessions of ST in patients with cMDD using a multiple-baseline single-case series design. We compared the effects of ST relative to a (quasi-random) baseline (i.e. waiting list) period in 25 patients with cMDD. The strength of this design above an open-trial design or a 
single-case series design without multiple baselines is that, just like in an RCT, symptomatic improvements during treatment can be attributed to the intervention rather than to the passing of time (Kazdin, 1982). In this respect, the baseline period in the current single-case series serves the purpose of a waiting-list control group in an RCT. An extra asset of the current study is that, in addition to the baseline period, we included an exploration phase during which no interventions aimed to change symptoms were used. The exploration phase thus served as an extra control condition enabling us to test if symptomatic improvements occur due to the intervention or due to non-specific factors such as receiving empathy or attention for one's problems (see Arntz, Sofi, \& van Breukelen, 2013, for a similar design).

\section{Methods}

\section{Participants}

Twenty-five patients with cMDD participated in this study. Patients were recruited from a specialized secondary mental health facility in the Netherlands (RIAGG Maastricht, The Netherlands). Inclusion criteria were a primary diagnosis of cMDD, defined as meeting the DSM-IV criteria for MDD for at least two years as assessed by the Structured Clinical Interview for DSM-IV Axis-I Disorders (SCID-I; First, Spitzer, Gibbon, \& Williams, 1997); a score of at least 20 on the Beck Depression Inventory second edition (BDI-II; Beck, Steer, \& Brown, 1996); and age between 18-65 years. Patients were excluded if they had a medical condition that might be correlated with depression either directly or through medication intake; had a DSM-IV current or past diagnosis of major depression with psychotic features; current or past bipolar disorder; current or past psychotic disorder; alcohol or drug dependence or a autism spectrum disorder, as assessed with the SCID-I. Patients with cluster-A or cluster-B personality disorders, as assessed with the Structured Clinical Interview for DSM-IV Axis-II Disorders (SCID-II), were also excluded. Additional exclusion criteria were acute suicide risk and inability to speak and read the Dutch language. Patients taking antidepressant medication were excluded, unless they were stable on medication for at least three months prior to the screening procedure.

16 The study was approved by the medical ethical committee of the University Hospital Maastricht, The Netherlands and the local ethics committee of the specialized secondary mental health facility where treatment was provided (RIAGG Maastricht). All patients enrolled in the study signed written informed consent. Prior to patient recruitment, this study has been registered at clinicaltrials.gov (identifier: NCT01153867).

The study took place between December 2010 and March 201417. Table 1 provides an overview of the demographic and clinical characteristics of the sample.

\footnotetext{
${ }^{16}$ Medication dosage of one participant taking venlafaxine was increased from $75 \mathrm{mg}$. to $150 \mathrm{mg}$. two month prior to start of the study.

${ }^{17}$ At the end of the funding period for this study 7 patients out of the 25 patients who originally started with the study were still in treatment, receiving low-frequency ST (about 1 session per month). For the
} 
Table 1. Overview of the demographic and clinical characteristics of the sample

\begin{tabular}{|c|c|}
\hline Variable & Mean (SD) / Frequency (\%) \\
\hline Age & $41.36(16.45)$ \\
\hline $\begin{array}{l}\text { Gender } \\
\text { Female } \\
\text { Male }\end{array}$ & $\begin{array}{l}20(80 \%) \\
5(20 \%)\end{array}$ \\
\hline $\begin{array}{l}\text { Country of origin } \\
\text { Netherlands } \\
\text { Other }\end{array}$ & $\begin{array}{l}23(92 \%) \\
2(8 \%)\end{array}$ \\
\hline $\begin{array}{l}\text { Marital status } \\
\text { Single } \\
\text { Partner } \\
\text { Married } \\
\text { Divorced }\end{array}$ & $\begin{array}{l}10(40 \%) \\
6(24 \%) \\
7(28 \%) \\
2(8 \%)\end{array}$ \\
\hline $\begin{array}{l}\text { Education level } \\
\text { Low } \\
\text { Medium } \\
\text { High }\end{array}$ & $\begin{array}{l}1(4 \%) \\
8(32 \%) \\
16(64 \%)\end{array}$ \\
\hline $\begin{array}{l}\text { Work Situation } \\
\text { Paid work or student } \\
\text { Unemployed } \\
\text { Retired } \\
\text { Other }\end{array}$ & $\begin{array}{l}10(40 \%) \\
3(36 \%) \\
9(36 \%) \\
3(12 \%)\end{array}$ \\
\hline $\begin{array}{l}\text { BDI-II score at screening } \\
\text { \# previous episodes }\end{array}$ & $\begin{array}{l}32.96(9.37) \\
2.80(2.14)\end{array}$ \\
\hline $\begin{array}{l}\text { Secondary Axis-I diagnosis } \\
\text { None } \\
\text { Anxiety Disorder } \\
\text { Other }\end{array}$ & $\begin{array}{l}14(56 \%) \\
6(24 \%) \\
5(20 \%)\end{array}$ \\
\hline $\begin{array}{l}\text { Secondary Axis-II diagnosis } \\
\text { None } \\
\text { Depressive PD } \\
\text { Avoidant PD } \\
\text { Obsessive Compulsive PD } \\
\text { Dependent PD }\end{array}$ & $\begin{array}{l}9(32 \%) \\
9(36 \%) \\
7(28 \%) \\
6(24 \%) \\
1(4 \%)\end{array}$ \\
\hline Antidepressant use at baseline & $11(44 \%)$ \\
\hline
\end{tabular}

Note. BDI-II = Beck Depression Inventory - second edition.

\section{Design}

We used a single case series design with multiple quasi random baselines across participants and 12 months follow-up (follow-up data not reported here). The study consisted of three phases: Phase 1: The baseline phase was a $6-24$ weeks waiting period that served as a no-treatment control phase. The duration of the baseline phase was quasi-random (dependent on the waiting time at the clinic). Phase 2: The 
exploration phase lasted 12 weeks and consisted of 8 to 10 sessions that served as an extra control for receiving attention and empathy. The number of sessions in the exploration phase varied depending on individual progress in identifying and exploring underlying schemas. Phase 3: During the intervention phase, participants received up to 65 individual sessions ST as described below. Therapists were instructed to not exceed a total of 75 sessions (exploration + intervention phase). During all study phases all primary and secondary outcomes were repeatedly assessed.

\section{Assessments}

\section{Primary outcome measures}

\section{Response, Remission and Recovery from depression}

Treatment response was defined as a 50\% drop in depressive symptom severity, as assessed with the BDI-II, from the first baseline assessment to the last intervention assessment. Remission was defined as a mean score of six or lower on the QIDS-SR at the last intervention phase assessment, in line with a previous study in patients with chronic depression that compared QIDS-SR cutoffs of remission with established cutoffs on the Hamilton Rating Scale of Depression (Rush et al., 2003). We defined recovery from depression as the absence of a DSM-IV diagnosis of depression at post-treatment as assessed with the SCID-I interview. SCID-I post intervention interviews of 12 patients who completed treatment were available.

\section{Continuous primary outcome measures}

BDI-II - The Beck Depression Inventory, second edition, was used to measure depressive symptom severity (Beck et al., 1996). Each of the 21-items of the BDI-II consists of four statements with increasing severity ranging from 0 to 3 , resulting in a total score of 0 to 63 with higher scores representing more depressive symptom severity. The instructions of the original BDI-II were adapted in the current study, asking participants to evaluate depressive symptoms during the past week instead of the past two weeks because the BDI-II was administered once a week. The Dutch version of the BDI-II that was used in the present study has been shown to have good psychometric properties (van der Does, 2002). In the current study, the internal reliability coefficient alpha of the BDI-II at the initial baseline assessment was excellent $(\alpha=.91)$.

QIDS-SR - The Quick Inventory of Depressive Symptomatology self-report version is a 16-item self-report instrument measuring depressive symptom severity over the past seven days (Rush et al., 2003). This shortened version of the original 30-item version (Rush, Gullion, Basco, Jarrett, \& Trivedi, 1996) has been shown to highly correlate with clinical rating scales, such as the Hamilton Rating Scale for Depression, in patients with chronic depression (Rush et al., 2003). In the current study, the internal reliability coefficient alpha of the QIDS-SR at the initial baseline assessment was satisfactory $(\alpha=.75)$. 


\section{Continuous secondary outcome measures}

$B S I$ - The Brief Symptom Inventory is a 53-items self-report instrument measuring symptomatic distress along nine subscales (Derogatis, 1992). In the current study, a global severity index was calculated by averaging all scores. In the original BSI, participants rate each item along a 5-point scale to describe whether an item applies to them or not. In the present study the original scale was adapted to a $100 \mathrm{~mm}$ Visual Analogue Scale (VAS) for two reasons: First, VAS scales might be more sensitive to subtle changes because respondents are not forced into a response category (Breivik, Bjornsson, \& Skovlund, 2000). Second, we were concerned that due to the large number of repeated assessments participants might remember response categories which might introduce a socially desirable answering bias. Good psychometric properties have been reported for the BSI (de Beurs, 2004). In the current study, the internal reliability coefficient alpha of the BSI at the initial baseline assessment was excellent ( $\alpha=.94)$.

ORS - The outcome rating scale (ORS) is a short, 4-item, self-report instrument that was designed to assess client functioning along three areas: individual functioning, interpersonal functioning, and social functioning (Miller, Duncan, Brown, Sparks, \& Claud, 2003). In addition to these areas the questionnaire includes an item assessing overall wellbeing. In the current study the overall wellbeing scale was used in all analyses. The ORS has been shown to possess adequate psychometric properties (Miller et al., 2003).

\section{Procedure}

\section{Schema Therapy for chronic depression}

Figure 1 provides an overview of the assessments during the different study phases. Patients completed the BDI-II and the ORS weekly during the baseline phase, the exploration phase and the intervention phase. The QIDS-SR and the BSI were completed on a monthly basis. These questionnaires were completed less frequent to decrease the burden of repeatedly completing lengthy questionnaires. The initial baseline assessment was conducted at the university. All remaining questionnaires were completed via the internet: Participants received an email once a week, on a fixed date, with personal login information for a secured website. On the website participants completed all questionnaires. The SCID-I and SCID-II was completed during intake and at the end of treatment in order to determine the diagnostic status of participants. The SCID interviews at intake were conducted by experienced clinicians at the mood disorders unit of the clinical site where treatment was provided. SCID interviews at the end of treatment were conducted by the therapists. 


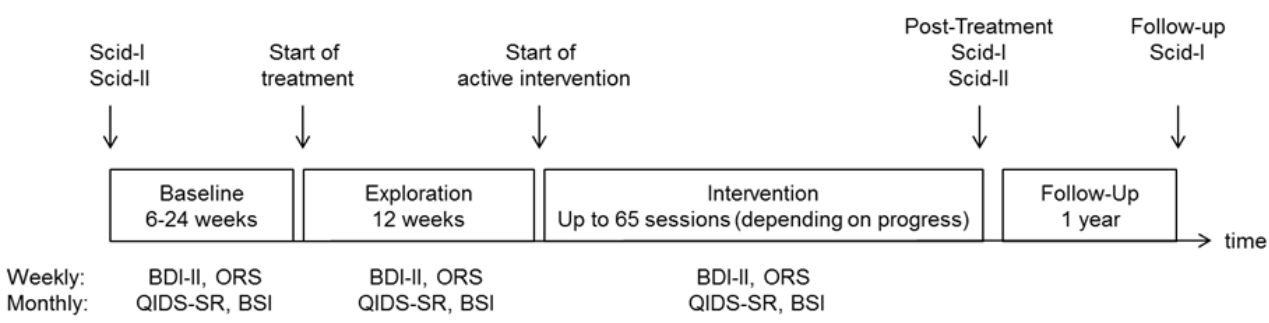

Figure 1. Overview of the assessments in the different study phases. SCID-I/II = Structured Clinical Interview for DSM-IV Axis-I/II Disorders; BDI-II = Beck Depression Inventory - second edition; BSI = Brief Symptom Inventory; ORS = Outcome Rating Scale; QIDS-SR = Quick Inventory of Depressive Symptomatology - Self Report.

The ST intervention consisted of 8 to 10 sessions exploration and up to 65 sessions active intervention with a combined maximum of 75 sessions lasting 50 minutes each. The definite number of sessions was dependent on the individual progress in therapy, in agreement between the patient and therapist. Therapy was provided in weekly sessions initially with lower frequency sessions in later stages of treatment. Therapy was conducted by experienced therapists (experience with schema therapy varying between 5 and 12 years) according to the methods described by Young et al. (2003). Treatment in this study was divided into two phases, the exploration phase and the intervention phase. During the exploration phase the therapist helped the patient to understand the schema concept and to establish relations between the patients' predominant schemas, current problems and his/her developmental history. The therapist also helped the patient to experience the feelings that are associated with the activation of these schemas. Thus during the exploration phase schemas and current problems are merely explored and no attempts are made to actively change schemas or resolve current problems. The intervention phase consisted of a maximum of 65 individual sessions of ST to work on change in underlying early maladaptive schemas, emotional experiences and dysfunctional behaviors. During this phase the underlying schemas are targeted by the use of cognitive techniques, experiential techniques, the therapeutic alliance (limited reparenting), and behavioral techniques (Young et al., 2003). Towards the end of the intervention phase a relapse prevention plan is established which includes an analysis of situations and events that could potentially trigger future depressive relapses. Coping strategies to deal with these situations are also included in the plan. A more detailed description of the treatment manual of ST for chronic depression can be found elsewhere (Renner et al., 2013) and a session-by-session protocol (in Dutch) is available from the first author.

\section{Statistical analyses}

Change in continuous primary and secondary outcome measures during the different treatment phases was modelled using mixed regression analyses in SPSS version 22. All available data was used in the analyses including data of participants who only provided baseline data for intent-to-treat principles and to increase the statistical power. 
Statistical analyses of baseline and treatment effects were based on the methods described in (Arntz et al., 2013; Malogiannis et al., 2014).

First, we analyzed change in depressive symptoms during the baseline phase to determine whether symptom severity remained stable in the absence of active treatment. To determine the optimal model we compared the model fit for different autoregressive covariance structures (AR1 and ARMA1,1) and different representations of change over time (linear, quadratic, logarithmic). The optimal model for the main continuous outcome measure (BDI-II) had an ARMA1,1 covariance structure for the repeated part with a logarithmic representation of time in the fixed effects part of the model and included random intercepts and slopes in the random part. Change over time was best represented by log-transformed time, thus the fixed effect of log-transformed time was used to test time development of depressive symptoms during baseline.

Next, we tested treatment effects of the exploration and intervention phase versus the baseline phase in terms of average scores within the phases and linear change within the phases. The analytic approach is based on the following fixed effects: a general time effect, additional dummy coded variables for the exploration and treatment phases as a test of differences in average symptom levels within phases relative to baseline, and linear time variables, individually centered within each phase as a test of change in depressive symptoms (in addition to the general time effect) during the exploration phase and intervention phase. Again logarithmic transformed time represented the general time effect best, so the general time effect was based on logarithmic transformation of time effect coded 0 when the first assessment took place for an individual. In these models, random intercepts were added but random slopes were left out due to convergence problems. When the general time effect was non-significant in the full model it was deleted and the model was re-run without the general time effect.

To determine the strength of treatment effects we calculated two version of Cohen's $d$. One based on the estimates of the mixed regression analyses by subtracting the last estimated treatment value from the last estimated baseline value and by dividing the result by the SD based on the variance of the random part of the model. The other version of Cohen's $d$ was obtained by subtracting the last observed value of the last treatment assessment from the last observed baseline value and by dividing the result by the SD of the last observed baseline assessment. The same approach was used to calculate effect sizes based on the last assessment in the exploration phase.

\section{Results}

\section{Description of the data}

Overall, there were 2022 weekly assessment moments across the study phases. Participants completed 1557 weekly assessments (77\% completion rate). On average, each participant received 10 weekly assessments during baseline (completion rate 93.1\%), 12 weekly assessments during the exploration phase (completion rate 89.2\%), and 78 weekly assessments during the intervention phase (completion rate $72.5 \%$ ). 
Thus, during the course of the entire study (excluding the follow-up phase), on average, participants received 100 weekly assessment batteries.

\section{Number of treatment sessions}

On average, patients in this study received 52.6 sessions ST (combined exploration and intervention phase; $\mathrm{SD}=14.01 ; \min =24 \mathrm{max}=74$ ). Patients who completed treatment on average completed 54.6 sessions ( $S D=14.56)$ whereas patients who were still in treatment at the end of the study period completed on average 48.71 sessions (SD = 13.09).

\section{Dropout}

Of the 25 patients recruited for this study 5 (20\%) dropped out during the baseline period. Three patients stopped with the study because they decided to start with a symptom focused treatment approach during the waiting time (two CBT; one antidepressants), one stopped because of a new job and lack of time and one stopped due to unspecified personal reasons. None of the 20 patients who started with treatment dropped out during the exploration or intervention phase.

\section{Primary outcomes}

\section{Response, Remission and Recovery}

At the last intervention phase assessment, 8 out of 20 (40\%) patients responded to treatment, defined as a $50 \%$ reduction in depressive symptom severity on the BDI-II. Of the 20 patients who started treatment, 7 (35\%) reached remission, defined as a score of 6 or lower on the QIDS-SR (Rush et al., 2003). Recovery from depression was defined as an absence of a DSM-IV diagnosis of depression at post-treatment. DSM-IV diagnoses as assessed with the SCID-I interview at post-treatment were available from 12 patients. The remaining 8 patients were still in treatment and therefore had no post treatment SCID data. Of the 12 patients with available DSM-IV diagnoses, seven (58\%) recovered.

\section{Change in depression severity during baseline}

For all analyses of baseline effects we first determined the optimal representation of the time variable by comparing model fit for a linear, quadratic and log-linear representation of time. The log-linear time variable provided the best fit to the data for both the BDI-II total score as well as for the QIDS-SR total score. There was a statistically significant logarithmic decrease in the BDI-II total score during baseline, B $=-2.03$, $t(25.83)=-2.56, p=.02$, suggesting that depressive symptom severity decreased during the baseline phase. Figure 2 is a graphical representation of the change in symptoms during baseline based on the mixed regression estimates. As can be seen in Figure 2, there was a relatively steep decrease in symptoms during the first weeks of baseline which then leveled out during the course of time, such that at the end of the baseline 
phase the average BDI-II score was still well above the cut-off at which patients were included. The effect size of change in BDI-II within the baseline phase was $d=0.39$ for the observed values and $d=0.51$ for the MLM derived estimates. The effect of logtransformed time for the QIDS-SR was also significant during baseline, $\mathrm{B}=-1.68, t(27.87)$ $=-2.81, p=.01$, showing that participants' QIDS-SR total score decreased initially during baseline before stabilizing again. The effect size of change in QIDS-SR within the baseline phase was $d=0.26$ for the observed values and $d=0.27$ for the MLM derived estimates.

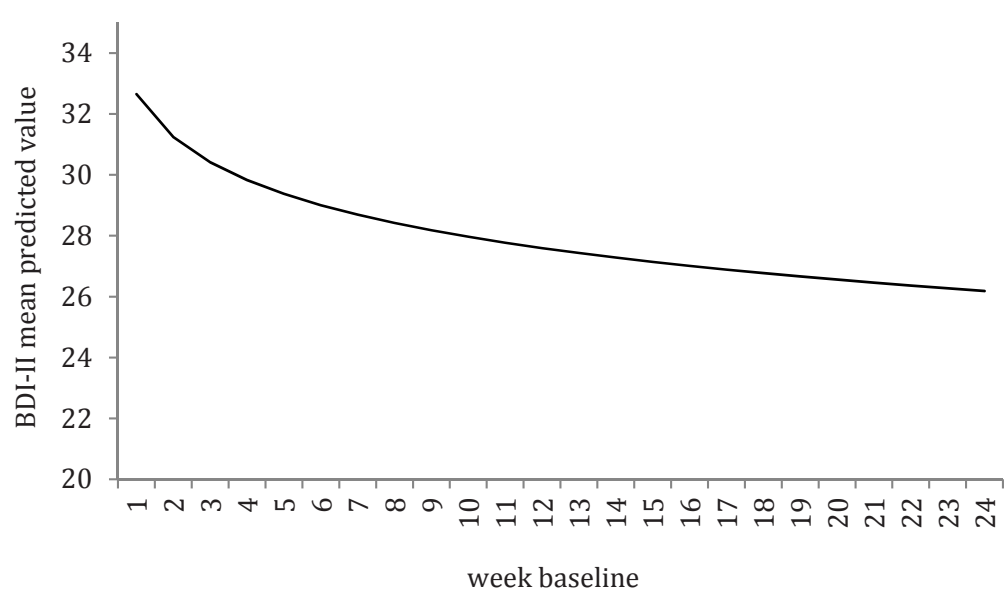

Figure 2. Graphical representation of decrease in depressive symptoms during baseline.

\section{Treatment effects on depressive symptom severity}

First, we tested a model with a general log time effect across phases as a single predictor using an ARMA1,1 covariance structure for the repeated part and random intercepts with the BDI-II total score as dependent variable. There was a significant effect of time (log-transformed), $\mathrm{B}=-2.63, t(112.08)=-6.38, p<.001$, suggesting that depressive symptoms decreased over time across phases. Next, we added dummy variables for condition, and linear time-within-phase (exploration and intervention) ${ }^{18}$ centered covariates to test for treatment effects (i.e. the slope within these phases relative to the slope of the general time effect). The results of these analyses are summarized in Table 2. In these analyses, the overall effect of log-transformed time became weaker, but remained significant, $\mathrm{B}=-2.31, t(567.46)=-3.67, p<.001$. The time-intervention effect was significant, $\mathrm{B}=-0.07, t(68.95)=-2.75, p=.008$, reflecting that the intervention had a significant impact on change in depressive symptoms that exceeded the overall decrease

\footnotetext{
${ }^{18}$ Due to the inclusion of the overall time effect in the model it is usually redundant to also include a timewithin baseline covariate in these analyses. However, when the time-within baseline covariate was included in the model that also included the general time effect, the effect of time within intervention remained significant $(p=.005)$. The addition of this linear time-within baseline effect was statistically possible because the general time effect was based on log-transformed time.
} 
in symptoms over time. The time-exploration phase effect was not significant, $B=0.14$, $t(385.99)=0.81, p=.42$, suggesting that depressive symptoms did not change during the exploration phase. Figure 3 plots the MLM predicted means for the BDI-II across the different study phases.

Effect size estimates (Cohen's $d$ ) for treatment effects were calculated by subtracting the last intervention phase assessment from the last baseline assessment and by dividing the result by the standard deviation. Based on the MLM estimates the effect size was $d=1.30$ and based on the observed mean and SD at the last baseline assessment the effect size was $d=0.91$. Based on the last exploration phase assessment effect size estimates for treatment effects were $d=1.42$ and $d=0.95$ for the MLM based and observed means and SDs, respectively.

We also tested a general time effect across phases for the QIDS-SR, using an ARMA1,1 covariance structure. Before adding dummy variables for condition, and timewithin-phase centered covariates, there was a significant effect of time, $\mathrm{B}=-1.54$, $t(95.83)=-4.28, p<.001$, reflecting that depressive symptoms on the QIDS-SR decreased over time (not reported in Table). After adding dummy variables for condition, and time-within-phase centered covariates, to test for treatment effects, the general time effect became non-significant, indicating that the general time effect was actually caused by the intervention, as the effect of linear change within the intervention phase was significant, $\mathrm{B}=-0.18, t(47.40)=-2.30, p=.03$, reflecting a decrease in QIDS-SR during intervention. All other effects were non-significant (Table 2). After removing the nonsignificant general time effect from the model the intervention time effect remained significant, $B=-.25, t(44.28)=-4.04, p<.001$, (Table 2$)$. The MLM predicted means of the QIDS-SR across the different treatment phases is shown in Figure 3.

Based on the MLM estimates of the model including the general time effect, the effect size for the QIDS-SR was $d=1.22$ and based on the observed mean and SD at the last baseline assessment the effect size was $d=1.20$. Based on the last exploration phase assessment effect size estimates for treatment effects were $d=1.23$ and $d=0.93$ for the MLM based estimates and observed means and SDs, respectively.

\section{Secondary outcomes}

As secondary continuous outcomes, we tested the effects of ST on general symptomatic distress and overall wellbeing. As in the primary analyses for continuous outcomes we first tested change in these variables during the baseline phase before testing for intervention effects. 
Table 2. Results of mixed regression analyses testing the effects of schema therapy on primary outcome measures.

\begin{tabular}{llllll}
\hline & Beta & $S E$ & $D F$ & $t$ & $p$ \\
\hline BDI-II & & & & & \\
$\quad$ Intercept & 32.92 & 2.32 & 41.47 & 14.19 & $<.001$ \\
Time $^{1}$ & -2.31 & 0.63 & 567.46 & -3.67 & $<.001$ \\
Exploration & 2.31 & 1.20 & 505.93 & 1.93 & .06 \\
$\quad$ Intervention & -0.88 & 2.03 & 344.49 & -0.44 & .66 \\
Time Exploration & 0.14 & 0.17 & 385.99 & 0.81 & .42 \\
Time Intervention & -0.07 & 0.02 & 68.95 & -2.75 & .008 \\
QIDS-SR including general time effect & & & & & \\
Intercept & 14.94 & 1.11 & 39.31 & 13.51 & $<.001$ \\
Time ${ }^{1}$ & -1.22 & 0.71 & 293.91 & -1.72 & .09 \\
Exploration & 0.81 & 0.89 & 234.95 & 0.92 & .36 \\
Intervention & -1.29 & 1.61 & 244.13 & -0.80 & .43 \\
Time Exploration & 0.81 & 0.97 & 290.52 & 0.84 & .40 \\
Time Intervention & -0.18 & 0.08 & 47.40 & -2.30 & .03 \\
QIDS-SR after deleting ns. general time effect & & & & & \\
Intercept & 14.27 & 1.03 & 31.69 & 13.79 & $<.001$ \\
Exploration & -0.35 & 0.58 & 234.32 & -0.60 & .55 \\
Intervention & -3.69 & 0.77 & 55.69 & -4.78 & $<.001$ \\
Time Exploration & 0.26 & 0.91 & 308.50 & 0.28 & .78 \\
Time Intervention & -0.25 & 0.06 & 44.28 & -4.04 & $<.001$ \\
\hline
\end{tabular}

Note. 1 = Log transformed

Change in global symptom severity (BSI)

First we tested change in global symptom severity during baseline as assessed with the BSI. Change in global symptom severity during baseline was best described by a logtransformed-time model. There was a significant decrease in global symptom severity during baseline, $\mathrm{B}=-3.76, t(37.82)=-2.04, p=.048$. Next, we entered the dummy coded variables for the exploration and intervention phase as well as the time-within-phase centered variables as a test of linear change in global symptom severity within the different phases, relative to the baseline phase. In this model, the general effect of time was not significant, $B=-2.96, t(307.20)=-1.52, p=.13$ and neither was the time-within intervention phase fixed effect, $\mathrm{B}=-0.36, t(82.38)=-1.73, p=.09$. However, after removing the non-significant general time effect from the model, there was a significant effect of time-within intervention phase, $\mathrm{B}=-0.54, t(32.53)=-3.24, p=.003$, indicating that general symptom severity decrease within the intervention phase was responsible for the overall decrease. Change in the general symptom severity score across the different study phases is displayed in Figure 3. 
Based on the MLM estimates of the model excluding the non-significant general time effect, the effect size for the BSI was $d=0.86$ and based on the observed mean and SD at the last baseline assessment the effect size was $d=0.64$. Based on the last exploration phase assessment effect size estimates for treatment effects were $d=0.76$ and $d=0.44$ for the MLM based estimates and observed means and SDs, respectively.

\section{Change in overall wellbeing (ORS)}

First, we investigated change in overall wellbeing during baseline. As for depressive symptom severity, change in overall wellbeing during baseline was best described by log-transformed time. There was a significant effect of time during baseline on overall wellbeing, $\mathrm{B}=4.28, t(58.13)=2.15, p=.04$, indicating that overall wellbeing improved during the initial baseline assessments and stabilized subsequently. To test the effects of the intervention phase on overall wellbeing, we entered log-transformed time, the dummy coded variables for the exploration and intervention phase as well as the timewithin-treatment phase centered variables as a test of linear change in overall wellbeing within the different phases. In this model, the general effect of time was not significant, B $=3.00, t(560.50)=1.69, p=.09$. The results of these analyses are summarized in Table 3 . There was a significant effect of the intervention phase on improvements in overall wellbeing, $\mathrm{B}=0.10, t(219.37)=2.17, p=.03$, indicating that the intervention had a significant impact on improved overall wellbeing that exceeded the overall decrease in symptoms over time. After deleting the non-significant general time effect, the time effect of the intervention phase remained significant, $B=0.15, t(129.37)=4.20, p<.001$, (Table 3). Change in overall wellbeing across the different study phases is shown in Figure 3.

Based on the MLM estimates of the model excluding the non-significant general time effect, the effect size for improvements in overall wellbeing was $d=0.93$ and based on the observed mean and SD at the last baseline assessment the effect size was $d=0.96$. Based on the last exploration phase assessment effect size estimates for treatment effects were $d=0.98$ and $d=1.12$ for the MLM based estimates and observed means and SDs, respectively. 
Table 3. Results of mixed regression analyses testing the effects of schema therapy on secondary outcome measures.

\begin{tabular}{|c|c|c|c|c|c|}
\hline & Estimate & $S E$ & $D F$ & $t$ & $P$ \\
\hline \multicolumn{6}{|l|}{$B S I$} \\
\hline Intercept & 48.64 & 3.25 & 36.76 & 14.97 & $<.001$ \\
\hline Time $^{1}$ & -2.96 & 1.94 & 313.67 & -1.52 & .13 \\
\hline Exploration & 2.32 & 2.41 & 328.83 & 0.96 & .34 \\
\hline Intervention & -2.07 & 4.39 & 277.91 & -0.47 & .64 \\
\hline Time Exploration & -1.00 & 2.56 & 326.30 & -0.39 & .70 \\
\hline Time Intervention & -0.36 & 0.21 & 86.02 & -1.73 & .09 \\
\hline \multicolumn{6}{|c|}{ BSI after deleting ns. general time effect } \\
\hline Intercept & 47.11 & 3.07 & 29.97 & 15.36 & $<.001$ \\
\hline Exploration & -0.47 & 1.58 & 233.61 & -0.30 & .77 \\
\hline Intervention & -7.95 & 2.09 & 30.85 & -3.81 & .001 \\
\hline Time Exploration & -2.37 & 2.41 & 318.75 & -1.00 & .33 \\
\hline Time Intervention & -0.54 & 0.17 & 32.53 & -3.24 & .003 \\
\hline \multicolumn{6}{|l|}{ ORS } \\
\hline Intercept & 36.00 & 3.91 & 130.40 & 9.20 & $<.001$ \\
\hline Time $^{1}$ & 3.00 & 1.78 & 560.50 & 1.69 & .09 \\
\hline Exploration & -2.03 & 3.27 & 509.18 & -0.62 & .53 \\
\hline Intervention & 5.44 & 5.05 & 403.39 & 1.08 & .28 \\
\hline Time Exploration & -0.3 & 0.47 & 568.61 & -0.66 & .51 \\
\hline Time Intervention & 0.10 & 0.05 & 219.37 & 2.17 & .03 \\
\hline \multicolumn{6}{|c|}{ ORS after deleting ns. general time effect } \\
\hline Intercept & 40.32 & 2.96 & 46.84 & 13.63 & $<.001$ \\
\hline Exploration & 1.59 & 2.47 & 397.28 & 0.65 & .52 \\
\hline Intervention & 13.10 & 2.21 & 177.91 & 5.91 & $<.001$ \\
\hline Time Exploration & 0.01 & 0.43 & 774.10 & 0.03 & .98 \\
\hline Time Intervention & 0.15 & 0.04 & 129.37 & 4.20 & $<.001$ \\
\hline
\end{tabular}

Note. ${ }^{1}$ Log transformed 


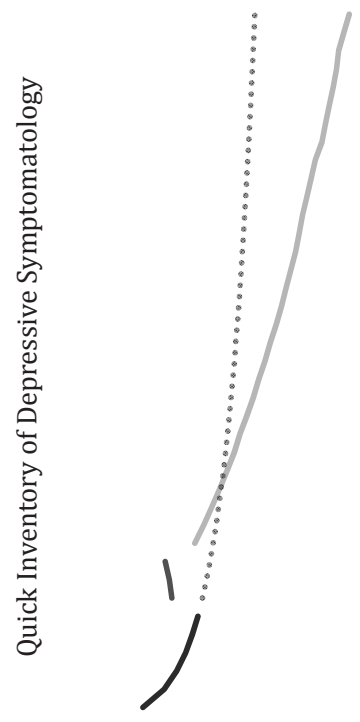

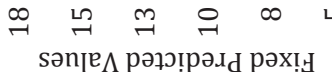

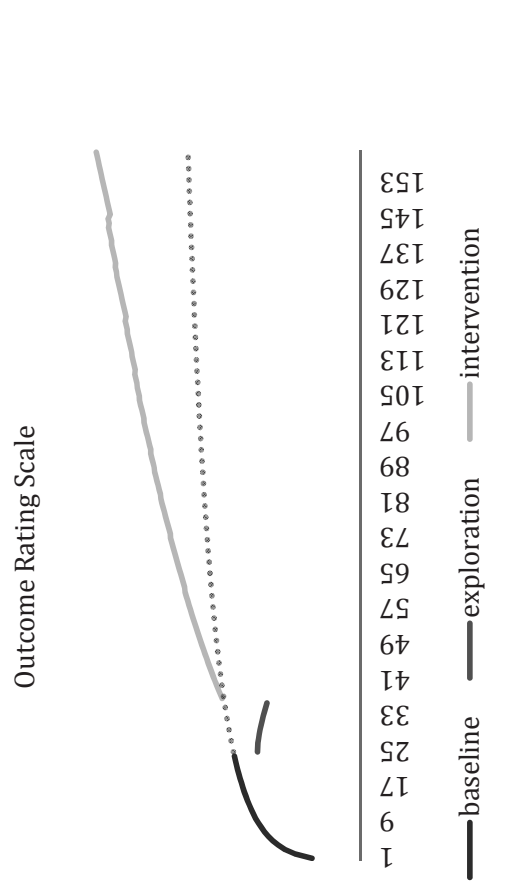

능 으는 은 낭워 눙

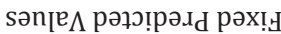

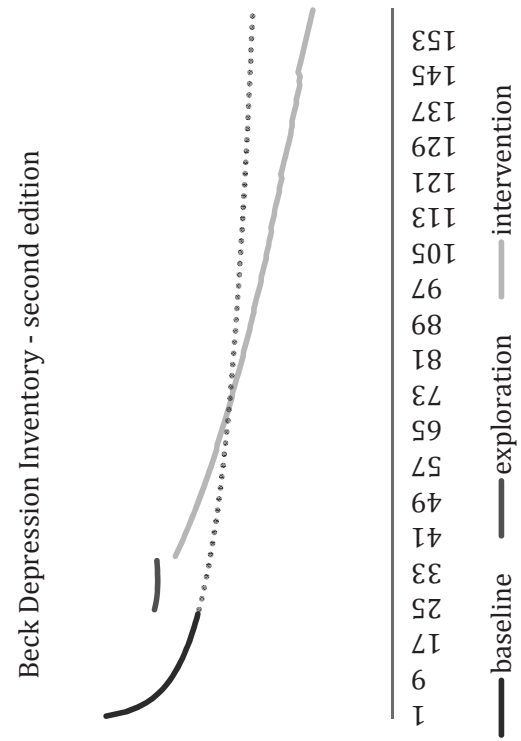

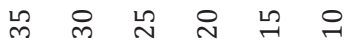

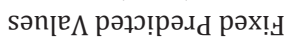

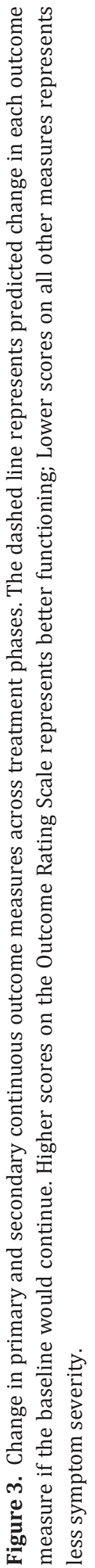




\section{Discussion}

The aim of this study was to test the effects of individual ST for cMDD on depressive symptoms, recovery from depression and improvements in overall wellbeing. We found that $40 \%$ of the patients responded to treatment whereas $35 \%$ remitted. Of the subsample of 12 patients who were reassessed with the SCID after treatment completion, 58\% did not meet the DSM-IV criteria for a depressive disorder at posttreatment anymore and were therefore considered to have recovered from depression. Relative to the overall decrease in symptoms over time, there was a significant effect of the ST intervention phase on depressive symptoms. After accounting for decreased symptom severity during the baseline phase, by considering the last baseline phase assessments, the effect sizes for decreased depressive symptoms during the intervention phase were large. The exploration phase had no effects on depressive symptom severity.

Although five patients dropped out during the baseline phase of the study, none of the patients in the current study dropped out from treatment. Previous studies of ST for personality disorder have also reported relatively lower dropout rates in ST compared to treatment as usual (Bamelis et al., 2013) or psychotherapy control conditions (Giesen-Bloo et al., 2006). ST places special emphasis on building a strong therapeutic relationship during the exploration phase (Young et al., 2003). Although speculative, it is possible that this protects from dropout during treatment because patients might feel more attached and committed to the therapy. In line with this, it has been shown that negative ratings of the therapeutic alliance predict dropout, and that the high treatment retention of ST probably relates to early strong therapeutic alliance (Spinhoven, GiesenBloo, van Dyck, Kooiman, \& Arntz, 2007).

Overall, our findings add to the accumulating evidence that ST is a promising new treatment for patients with (chronic) depression (Carter et al., 2013; Malogiannis et al., 2014). Malogiannis et al. (2014) conducted a single case series for chronically depressed women and found comparable remission (41.6\%) and recovery (60\%) rates. In their study, relatively larger effect sizes for ST were reported when contrasted with baseline, using a clinical rating scale (Hamilton Rating Scale for Depression) to assess depressive symptom severity. A recent meta-analysis has shown that clinical rating scales of depressive symptoms usually result in larger effect sizes (Cuijpers, Li, Hofmann, \& Andersson, 2010) and therefore differences in effect sizes in the current study can at least in part be attributed to differences in the outcome assessment format. Moreover, in the current study a more stringent control for change in symptoms over time was used, as we implemented multiple quasi-random baselines in our experimental design. Finally, in the study by Malogiannis et al. (2014) only 5 out of 12 patients fulfilled the criteria of cMDD, whereas the remaining patients had either a less severe form of chronic depression (dysthymic disorder) or an non-chronic depressive episode superimposed on a dysthymic disorder (i.e. double depression). Thus, differences in diagnostic groups might also account for differences in effect sizes obtained. 
Slightly higher remission rates were reported by (Carter et al., 2013) comparing the effects of a shorter ST protocol to cognitive therapy in a RCT. Half of the patients in the ST arm in the study by Carter and colleagues remitted. Carter et al. (2013) also included non-chronically depressed patients and although no differences were found for overall treatment effects between chronically depressed and non-chronically depressed patients, it is possible that the non-chronically depressed patients contributed to the slightly higher remission rates.

When benchmarking the findings of the current study against the effect size found in a recent meta-analysis of psychotherapy for chronic depression $(d=0.23$; Cuijpers, van Straten, et al., 2010), ST in the current study seems to perform clearly above average. Although the results of the current study cannot directly be compared to meta-analytic findings based on RCTs, the stringent control phases in the current study allowed us to control for a general decrease in symptoms over time (baseline phase) and for possible effects of receiving attention and empathy (exploration phase). In this sense, the control phases in the current study are comparable to waiting-list control conditions (baseline phase) and non-active control treatment (exploration phase) conditions in an RCT. Interestingly, in the current study the exploration phase had no effects on changes in depressive symptoms or secondary outcomes which indicates that non-specific therapy factors such as empathy, receiving attention and the development of a therapeutic relationship had no direct effect on symptomatic improvements. Symptom reports even suggest worsening during this phase, an effect previously observed by Weertman and Arntz (2007) in a study in patients with personality disorders. Note however that although the exploration phase had no direct effects on symptoms in the current study it is possible that the received attention and empathy and the therapeutic bond that is built during this phase might still have had an effect on symptom improvements in later stages of treatment.

We also tested for improvements in global symptomatic distress and overall wellbeing as relevant secondary outcomes. Participants improved with moderate to large effect sizes on global symptomatic distress, indicating that the positive effects of ST on depressive symptoms extended to broader aspects of distress. Improvements in overall wellbeing during the intervention phase were also large, indicating that the positive effects of ST on depressive symptoms transferred to improvements in overall wellbeing. Relatively smaller effect sizes for social functioning, an aspect of overall wellbeing, were reported in a recent meta-analysis testing the effects of psychotherapy for depression on psychosocial functioning, even when treatment was contrasted with primarily waiting-list control conditions (Renner, Cuijpers, \& Huibers, 2014), most closely resembling the baseline control phase in the current study.

The current study included a 6-24 weeks quasi-random baseline period. The purpose of this baseline period was to control for change in symptoms over time in the absence of active treatment. Unexpectedly, we found a significant, moderate, decrease in depressive symptom severity and in secondary continuous outcomes during baseline. This decrease could best be described by a log-linear curve, with a relatively strong initial decrease in symptoms which then leveled out during the course of the baseline 
period. At least two explanations might account for the decrease during baseline. 1) The decrease in symptoms during baseline represents a 'true' change in depressive symptom severity or 2) the decrease in symptoms during baseline represents a measurement artifact. In favor of the first explanation, it is possible, for example, that patients experienced a strong motivation to start with a novel, promising, treatment in an academic context. This might have led to an initial alleviation in depressive symptoms during the first weeks of the study (i.e. an expectancy effect) which then leveled out in the course of the baseline phase. In favor of the second explanation, it is possible that patients initially overestimated their symptom severity and that due to the repeated completion of and reflection on the questionnaires established a new, 'true', stable baseline. In line with the second explanation, it has been shown previously, that change in the BDI in the absence of treatment is a function of the frequency of test administration (Longwell \& Truax, 2005) with weekly administration resulting in decreases in the overall score whereas BDI scores remain stable during bi-weekly or monthly administration. Moreover, based on data drawn from the National Institute of Mental Health Treatment of Depression Collaborative Research Program (Elkin, Parloff, Hadley, \& Autry, 1985), it has been shown than patients with depression become more accurate in assessing their self-reported depressive symptom severity on the BDI over time (Fokkema, Smits, Kelderman, \& Cuijpers, 2013). Given that patients in the current study, by definition, suffered from depression for at least two years, it is unlikely that they experienced a 'true' decrease in symptoms during baseline. This interpretation is speculative and based on the current study design it is not possible to empirically support either explanation. To determine if the frequency of assessments of depressive symptom severity introduces a measurement error, patients should be randomized to different frequencies of repeated assessments in the absence of treatment.

Overall, the finding that depressive symptoms decreased during baseline has important implications for treatment outcome studies. Depressed patients who are included in treatment outcome studies should be re-assessed several times until symptom severity is stable. If studies fail to do so it is possible that part of the initial decrease in symptoms seen in the first weeks of treatment is due to a measurement artifact.

\section{Limitations}

A number of important limitations should be considered when interpreting these results. First, we conducted a single case series study which by design does not include a control group which can be perceived as a limitation. However, by including a non-treatment multiple baseline phase and an exploration phase, we were able to attribute decreased symptoms over time to the ST intervention. Second, the rather strong decrease in depressive symptoms during the baseline phase complicated the detection and estimation of treatment effect sizes substantially. For a case-series, symptom levels are ideally stable during the baseline phase. Due to the unstable baseline in the current study we have to (implicitly) rely on extrapolation of change assessed during the 
baseline to the exploration and intervention periods. Third, post-treatment SCID assessments were conducted by the therapists, who were not blind to the treatment and might therefore have been biased in their judgment.

In conclusion, the findings of this study add to the accumulating evidence that ST is an effective treatment for patients with cMDD. The fact that the two previous studies on ST for (chronic) depression and the current study were all conducted by independent research groups speaks to the generalizability of findings. The low dropout rates in previous ST studies and the absence of dropout in the current study highlight the acceptability of ST by patients. Although there is now emerging evidence that ST is an effective treatment for (chronic) depression, future RCT based studies in larger samples are needed to determine the efficacy of ST for chronic depression and the comparative efficacy of ST relative to other interventions.

\section{Acknowledgements}

The authors would like to thank the participating patients, therapists, research coordinators and research assistants. 


\section{References}

Arnow, B., \& Constantino, M. (2003). Effectiveness of Psychotherapy and Combination Treatment for Chronic Depression. Journal of Clinical Psychology, 59, 893 - 905.

Arntz, A., Sofi, D., \& van Breukelen, G. (2013). Imagery Rescripting as treatment for complicated PTSD in refugees: a multiple baseline case series study. Behaviour Research and Therapy, 51(6), 274-283. doi: 10.1016/j.brat.2013.02.009

Bamelis, L. L. M., Evers, S. M. A. A., Spinhoven, P., \& Arntz, A. (2013). Results of a multicentered randomized controlled trial on the clinical effectiveness of schema therapy for personality disorders. American Journal of Psychiatry. doi: doi:10.1176/appi.ajp.2013.12040518

Beck, A. T., Steer, R. A., \& Brown, G. K. (1996). Manual for the Beck Depression Inventory-II. San Antonio, TX.

Breivik, E. K., Bjornsson, G. A., \& Skovlund, E. (2000). A comparison of pain rating scales by sampling from clinical trial data. Clinical Journal of Pain, 16(1), 22-28.

Brewin, C. R., Wheatley, J., Patel, T., Fearon, P., Hackmann, A., Wells, A., . . Myers, S. (2009). Imagery rescripting as a brief stand-alone treatment for depressed patients with intrusive memories. Behaviour Research and Therapy, 47(7), 569-576. doi: 10.1016/j.brat.2009.03.008

Brown, G. W., Craig, T. K. J., Harris, T. O., Handley, R. V., \& Harvey, A. L. (2007). Development of a retrospective interview measure of parental maltreatment using the Childhood Experience of Care and Abuse (CECA) instrument -- A life-course study of adult chronic depression -- 1. Journal of Affective Disorders, 103(1-3), 205-215. doi: 10.1016/j.jad.2007.05.022

Brown, G. W., \& Moran, P. (1994). Clinical and psychosocial origins of chronic depressive episodes: I. A community survey. British Journal of Psychiatry, 165(4), 447-456. doi: 10.1192/bjp.165.4.447

Carter, J. D., McIntosh, V. V., Jordan, J., Porter, R. J., Frampton, C. M., \& Joyce, P. R. (2013). Psychotherapy for depression: a randomized clinical trial comparing schema therapy and cognitive behavior therapy. Journal of Affective Disorders, 151(2), 500-505. doi: 10.1016/j.jad.2013.06.034

Cuijpers, P., Li, J., Hofmann, S. G., \& Andersson, G. (2010). Self-reported versus clinician-rated symptoms of depression as outcome measures in psychotherapy research on depression: a meta-analysis. Clinical Psychology Review, 30(6), 768-778. doi: 10.1016/j.cpr.2010.06.001

Cuijpers, P., van Straten, A., Schuurmans, J., van Oppen, P., Hollon, S. D., \& Andersson, G. (2010). Psychotherapy for chronic major depression and dysthymia: A meta-analysis. Clinical Psychology Review, 30(1), 51-62. doi: 10.1016/j.cpr.2009.09.003

de Beurs, E. (2004). De Brief Symptom Inventroy: Handleiding [The Brief Symptom Inventroy: Manual]. Leiden: Pits Publishers.

Derogatis, L. R. (1992). The Brief Symptom Inventory (BSI); Administration, scoring and procedures manualII.: Clinical Psychometric Research Inc.

Elkin, I., Parloff, M. B., Hadley, S. W., \& Autry, J. H. (1985). NIMH Treatment of Depression Collaborative Research Program. Background and research plan. Archives of General Psychiatry, 42(3), 305-316.

Fava, M., Alpert, J., Borus, J., Nierenberg, A., Pava, J., \& Rosenbaum, J. (1996). Patterns of personality disorder comorbidity in early-onset versus late- onset major depression. American Journal of Psychiatry, 153(10), 1308-1312.

First, M. B., Spitzer, R. L., Gibbon, M., \& Williams, J. B. (1997). Structured Clinical Interview for DSM-IV Axis I Disorders (SCID-I). New York: Biometrics Research Department.

Fokkema, M., Smits, N., Kelderman, H., \& Cuijpers, P. (2013). Response shifts in mental health interventions: an illustration of longitudinal measurement invariance. Psychological Assessment, 25(2), 520-531. doi: 10.1037/a0031669

Giesen-Bloo, J., van Dyck, R., Spinhoven, P., van Tilburg, W., Dirksen, C., van Asselt, T., ... Arntz, A. (2006). Outpatient Psychotherapy for Borderline Personality Disorder: Randomized Trial of SchemaFocused Therapy vs Transference-Focused Psychotherapy. Archives of General Psychiatry, 63(6), 649-658. doi: 10.1001/archpsyc.63.6.649 
Hayden, E. P., \& Klein, D. N. (2001). Outcome of Dysthymic Disorder at 5-Year Follow-Up: The Effect of Familial Psychopathology, Early Adversity, Personality, Comorbidity, and Chronic Stress. American Journal of Psychiatry, 158(11), 1864-1870. doi: 10.1176/appi.ajp.158.11.1864

Holmstrand, C., Engström, G., \& Träskman-Bendz, L. (2008). Disentangling dysthymia from major depressive disorder in suicide attempters' suicidality, comorbidity and symptomatology. Nordic Journal of Psychiatry, 62(1), 25-31. doi: doi:10.1080/08039480801960164

Howland, R. H. (1993). Chronic depression. Hospital and Community Psychiatry, 44(7), 633-639.

Kazdin, A. E. (1982). Single-case research designs: Methods for clinical and applied settings. New York: Oxford University Press.

Keller, M. B., Hanks, D. L., Kocsis, J. H., \& Klein, D. N. (1995). Course and natural history of chronic depression Diagnosis and treatment of chronic depression. (pp. 58-72). New York, NY US: Guilford Press.

Kocsis, J. H. (2003). Pharmacotherapy for chronic depression. Journal of Clinical Psychology, 59(8), 885892. doi: $10.1002 / j \operatorname{clp} .10180$

Lizardi, H., \& Klein, D. N. (2000). Parental psychopathology and reports of the childhood home environment in adults with early-onset dysthymic disorder. The Journal of nervous and mental disease, 188(2), 63-70. doi: 10.1097/00005053-200002000-00001

Longwell, B. T., \& Truax, P. (2005). The differential effects of weekly, monthly, and bimonthly administrations of the beck Depression Inventory-II: Psychometric properties and clinical implications. Behavior Therapy, 36(3), 265-275. doi: http://dx.doi.org/10.1016/S00057894(05)80075-9

Malogiannis, I. A., Arntz, A., Spiropoulou, A., Tsartsara, E., Aggeli, A., Karveli, S., ... Zervas, I. (2014). Schema Therapy for patients with chronic depression: A single case series study. Journal of Behavior Therapy and Experimental Psychiatry, 45(3), 319-329. doi: http://dx.doi.org/10.1016/j.jbtep.2014.02.003

Miller, S. D., Duncan, B. L., Brown, J., Sparks, J. A., \& Claud, D. A. (2003). The Outcome Rating Scale: A Preliminary Study of the Reliability, Validity, and Feasibility of a Brief Visual Analog Measure. Journal of Brief Therapy, 2(2), 91 - 100.

Nadort, M., Arntz, A., Smit, J. H., Giesen-Bloo, J., Eikelenboom, M., Spinhoven, P., . . van Dyck, R. (2009). Implementation of outpatient schema therapy for borderline personality disorder with versus without crisis support by the therapist outside office hours: A randomized trial. Behaviour Research and Therapy, 47(11), 961-973. doi: 10.1016/j.brat.2009.07.013

Nordahl, H. M., \& Nysaeter, T. E. (2005). Schema therapy for patients with borderline personality disorder: a single case series. Journal of Behavior Therapy and Experimental Psychiatry, 36(3), 254-264.

Pepper, C., Klein, D., Anderson, R., Riso, L., Ouimette, P., \& Lizardi, H. (1995). DSM-III-R axis II comorbidity in dysthymia and major depression. American Journal of Psychiatry, 152(2), 239-247.

Renner, F., Arntz, A., Leeuw, I., \& Huibers, M. (2013). Treatment for chronic depression using schema therapy. Clinical Psychology Science and Practice, 20, 166-180.

Renner, F., Cuijpers, P., \& Huibers, M. J. H. (2014). The effect of psychotherapy for depression on improvements in social functioning: a meta-analysis. Psychological Medicine, FirstView, 1-14. doi: doi:10.1017/S0033291713003152

Renner, F., Lobbestael, J., Peeters, F., Arntz, A., \& Huibers, M. (2012). Early maladaptive schemas in depressed patients: Stability and relation with depressive symptoms over the course of treatment. Journal of Affective Disorders, 136(3), 581-590. doi: 10.1016/j.jad.2011.10.027

Rush, A. J., Gullion, C. M., Basco, M. R., Jarrett, R. B., \& Trivedi, M. H. (1996). The Inventory of Depressive Symptomatology (IDS): psychometric properties. Psychological Medicine, 26(03), 477-486. doi: doi:10.1017/S0033291700035558

Rush, A. J., Trivedi, M. H., Ibrahim, H. M., Carmody, T. J., Arnow, B., Klein, D. N., ... Keller, M. B. (2003). The 16-item Quick Inventory of Depressive Symptomatology (QIDS), clinician rating (QIDS-C), and selfreport (QIDS-SR): A psychometric evaluation in patients with chronic major depression. Biological Psychiatry, 54(5), 573-583. 
Smit, F., Cuijpers, P., Oostenbrink, J., Batelaan, N., de Graaf, R., \& Beekman, A. (2006). Costs of Nine Common Mental Disorders: Implications for Curative and Preventive Psychiatry. Journal of Mental Health Policy and Economics, 9(4), 193-200.

Spijker, J., van Straten, A., Bockting, C. L. H., Meeuwissen, J. A. C., \& van Balkom, A. J. L. M. (2013). Psychotherapy, antidepressants, and their combination for chronic major depressive disorder: A systematic review. The Canadian Journal of Psychiatry / La Revue canadienne de psychiatrie, 58(7), 386-392.

Spinhoven, P., Giesen-Bloo, J., van Dyck, R., Kooiman, K., \& Arntz, A. (2007). The therapeutic alliance in schema-focused therapy and transference-focused psychotherapy for borderline personality disorder. Journal of Consulting and Clinical Psychology, 75(1), 104-115. doi: 10.1037/0022006x.75.1.104

Torpey, D. C., \& Klein, D. N. (2008). Chronic depression: Update on classification and treatment. Current Psychiatry Reports, 10, 458-464.

van der Does, A. J. W. (2002). Handleiding: De Nederlandse versie van de Beck Depression Inventory, 2de Editie. [The Dutch version of the Beck Depression Inventory, 2nd ed.]. Lisse: Swets and Zeitlinger b.v.

Weertman, A., \& Arntz, A. (2007). Effectiveness of treatment of childhood memories in cognitive therapy for personality disorders: A controlled study contrasting methods focusing on the present and methods focusing on childhood memories. Behaviour Research and Therapy, 45(9), 2133-2143. doi: http://dx.doi.org/10.1016/j.brat.2007.02.013

Wells, K. B., Burnam, M. A., Rogers, W., \& Hays, R. (1992). The course of depression in adult outpatients: Results from the Medical Outcomes Study. Archives of General Psychiatry, 49(10), 788-794. doi: 10.1001/archpsyc.1992.01820100032007

Young, J. E., Klosko, J., \& Weishaar, M. E. (2003). Schema therapy: A practitioner's guide. New York: Guilford Press 


\section{CHAPTER 10 Exploring mechanisms of change in schema therapy for chronic depression}

Renner, F., DeRubeis, R., Arntz, A., Peeters, F., Lobbestael, J., \& Huibers, M. (2014). Exploring mechanisms of change in schema therapy for chronic depression: The role of negative core-beliefs and the therapeutic alliance. Manuscript in preparation. 


\begin{abstract}
Background: The underlying mechanisms of symptom change in schema therapy (ST) for chronic major depressive disorder (cMDD) have not been studied. Two potential key mechanisms underlying symptom change in ST are changes in schemas and the therapeutic alliance. One challenge in studying mechanisms of change is demonstrating that putative mechanisms precede change in symptoms.
\end{abstract}

Method: To disentangle the temporal relations between schemas (proxied by negative idiosyncratic core-beliefs), the therapeutic alliance and depressive symptoms we drew data from a single-case series of ST for cMDD. Patients with cMDD $(\mathrm{N}=20)$ received on average 78 repeated weekly assessments over a course of up to 65 individual sessions ST. Focusing on repeated assessments within-individuals, we tested whether change in core-beliefs and therapeutic alliance precedes, follows from or occurs concurrently with change in depressive symptoms. Weighted correlation coefficients and mixed regression were used to test each model.

Results: In general, change in core-beliefs was not temporarily but concurrently related to changes in symptoms. Overall alliance ratings were not temporarily related to symptom change. Repeated goal and task agreement ratings (specific aspects of alliance) of the same session, completed on separate days, were at least in part predicted by concurrent changes in symptoms.

Conclusion: Contrary to what would be expected based on theory, our findings suggest that change in core-beliefs does not precede change in symptoms. Instead, change in these variables occurs concurrently. Moreover, alliance ratings seem to be at least in part colored by changes in current mood state.

Keywords: schema therapy; chronic depression; negative core-beliefs; therapeutic alliance; mechanisms of change 


\section{Introduction}

Major depressive disorder (MDD) is a common mood disorder accounting for the greatest disease burden among mental disorders worldwide (Whiteford et al., 2013). Although effective treatments for MDD are available, it is estimated that about $20 \%$ of depressed patients do not recover within two years (e.g. Spijker et al., 2002). When symptoms of MDD are present for two years or longer depression is considered to be chronic (cMDD). Current treatments for cMDD include psychotherapy, antidepressants or their combination (for a review see: Spijker, van Straten, Bockting, Meeuwissen, \& van Balkom, 2013). Although psychotherapy is effective in treating cMDD, the effect sizes are rather small (Cuijpers et al., 2010) indicating that there is ample room for improvements. It has been argued that a better understanding of the underlying mechanisms that contribute to symptom change is the key to improving current treatments (Kazdin, 2007). The overall aim of the current study was to explore the impact of two potentially important mechanisms of symptom change, maladaptive schemas and the therapeutic alliance, during psychotherapy for cMDD

We have previously argued that current treatments for cMDD are too time-limited and might not adequately take the underlying vulnerability of cMDD into account (Renner, Arntz, Leeuw, \& Huibers, 2013). Schema therapy (ST) is a relatively new, longterm, integrative, treatment for chronic axis-I and axis-II disorders (Young, Klosko, \& Weishaar, 2003), with an established effectiveness for personality disorders (for a review see: Bamelis, Bloo, Bernstein, \& Arntz, 2012). An emerging body of evidence suggests that ST is also effective for cMDD (Carter et al., 2013; Malogiannis et al., 2014; Renner, Arntz, Peeters, Lobbestael, \& Huibers, 2014). We studied the effects of ST for cMDD in a single case-series and found that compared to a no-treatment control phase, as well as to an exploration phase, ST resulted in significant, large improvements in depressive symptom severity (Renner et al., 2014).

Although the evidence of ST for cMDD is emerging, the underlying mechanisms of ST for cMDD have not been investigated. The theoretical model of ST assumes that patients with psychological problems are characterized by a distinct set of early maladaptive schemas (Young et al., 2003). These schemas determine the way in which people perceive the world, themselves and others and can have a powerful impact on sensations, emotions and behavior (Young et al., 2003). The ultimate goal of ST is to decrease the impact of dysfunctional schemas and to replace them with more functional schemas. Theoretically, schemas might be related to depressive symptoms in at least three different ways: i) according to theory, change in schemas would be expected to drive subsequent change in symptoms during ST (Young et al., 2003), ii) contrary to theory, change in depressive symptoms drives subsequent change in schemas, iii) schemas change concurrently (i.e. at the same time) with depressive symptoms, suggesting that an unknown factor is driving change in both process and outcome.

Findings from previous studies that tested associations between schemas and change in symptoms during ST were mixed. Nordahl, Holthe, and Haugum (2005), for example, found that change in schemas from pre-treatment to post-treatment predicted 
improvements in global symptom severity in a mixed outpatient sample. In contrast, Renner, van Goor, et al. (2013) found that, in a sample of young adults with personality disorders (features) receiving a shortened group ST intervention, changes in global symptom severity accounted for changes in schemas, rather than the other way around. Importantly, these studies did not take the temporal relation between changes in schemas and symptoms into account and it therefore remains unclear whether change in schemas precedes change in symptoms or the other way around. Thus, one aim of the current study was to explore temporal associations between changes in schemas and changes in depressive symptoms during ST for cMDD.

Another potential key mechanism underlying symptom change in ST is the therapeutic alliance. The concept of the therapeutic alliance can be conceptualized as the degree to which client and therapist agree on the goals of treatment, the methods used to achieve these goals and the personal bond between client and therapist (Bordin, 1979). Research syntheses on the alliance - outcome relation have reported small to moderate correlations between the alliance and treatment outcome (e.g. Horvath, Del Re, Fluckiger, \& Symonds, 2011). Although the therapeutic alliance is usually considered a common factor in psychotherapy research, it might be an especially important contributor to outcome in ST (Spinhoven, Giesen-Bloo, van Dyck, Kooiman, \& Arntz, 2007). In ST the therapeutic alliance is used as a therapeutic technique, for example in limited reparenting in which the therapist takes the position as it were of a good parent to meet the unmet childhood needs of the client (Young et al., 2003). Similarly, imagery rescripting of traumatic experiences, a key technique in ST, likely requires a strong trust relationship between therapist and client (Hoffart, Oktedalen, Langkaas, \& Wampold, 2013). Theoretically, the therapeutic alliance might be related to change in symptoms in at least three different ways: i) the alliance rating of a session predicts change in symptoms from pre-session to post-session, ii) changes in symptoms prior to a session predict subsequent alliance ratings, or iii) two repeated alliance evaluations of the same session, completed at different times, might change concurrently (i.e. at the same time) with changes in symptoms, for example because the level of depression colors the evaluation. These possible alliance outcome relations have not been addressed sufficiently in the psychotherapy outcome literature. Thus, another aim of the current study was to explore temporal associations between the alliance and changes in symptoms during ST for cMDD.

It has been argued that previous interpretations of the alliance-outcome relationship are problematic due to correlational research designs that did not disentangle the temporal relation between change in symptoms and alliance assessments (DeRubeis, Brotman, \& Gibbons, 2005). Demonstrating temporal precedence between potential mechanisms and outcomes has been described as "the Achilles heel of treatment studies" ( Kazdin, 2007, p5). In order to demonstrate that a potential mechanism of symptom change precedes the observed effect in time it is necessary to establish a timeline (Kazdin, 2007). To establish a timeline it is necessary to assess symptoms and potential mechanisms of symptom change at multiple time points during treatment. We conducted a single case-series of ST for cMDD in which patients 
received on average 78 repeated weekly assessments during the intervention phase of the study, allowing us to map out the temporal relationships between changes in symptoms and potential mechanisms of symptom change. Assessments were completed weekly from home (online) and sessions were initially scheduled weekly, yet with lower frequencies later in treatment. Due to this large number of repeated assessments and the independence of assessments and sessions (assessments occurred independent of treatment sessions) we were able to establish a timeline and examine the underlying temporal relations between potential mechanisms (schemas and therapeutic alliance) and symptom change.

Repeated assessment of schemas is challenging because schemas are a highly theoretical and complex construct and usually assessed with an extensive questionnaire in ST (Young, 2006). In this study we used ratings of the strength of a set of idiosyncratic core-beliefs about five domains (self, other, world, future, and interpersonal relationships) as a proxy of underlying schemas. Acknowledging that in this way we assessed only a part of what a schema can entail, we therefore refer to this variable as "core-beliefs" instead of "schema".

The overall aim of the current study was to test potential mechanisms responsible for symptom change in ST for cMDD. Specifically, we tested temporal relations between change in core-beliefs and depressive symptoms and between (change in) the therapeutic alliance and depressive symptoms during ST for cMDD. We hypothesized that change in core-beliefs would predict subsequent symptom change rather than the other way around (hypothesis 1). Moreover, we hypothesized that change in symptoms from pre-session to post-session would be predicted by the alliance rating of that session (hypothesis 2). Finally, we hypothesized that change in depressive symptoms and change in core-beliefs would be concurrently related (hypothesis 3) as would be change in depressive symptoms and change in the therapeutic alliance (hypothesis 4). Note that the latter hypothesizes are not contradictory to the first two hypotheses as temporal and concurrent relations among these variables are not mutually exclusive. Formal mediation analyses (e.g., Kraemer, Wilson, Fairburn, \& Agras, 2002) are not of part of this study, as there was no control treatment to compare with.

\section{Method}

\section{Participants}

Twenty-five patients with a DSM-IV diagnosis of cMDD were included. Five patients stopped during the baseline period, before starting with the intervention. The current report is based on data from the remaining 20 patients who started with the intervention. Recruitment took place at a specialized secondary care facility in the Netherlands. Patients in the age range between 18-65 years, with a primary diagnosis of cMDD, defined as meeting the DSM-IV criteria for MDD for at least two years as assessed with the Structured Clinical Interview for DSM-IV Axis-I disorders (SCID-I; First, Spitzer, Gibbon, \& Williams, 1997) and a score of 20 or higher on the Beck Depression Inventory 
second edition (Beck, Steer, \& Brown, 1996) were included. Exclusion criteria were a current or past diagnosis of MDD with psychotic features; current or past bipolar disorder; current or past psychotic disorder; alcohol or drug dependence or autism spectrum disorder, as assessed with the SCID-I. Moreover, patients with a cluster-A or cluster-B personality disorder as assessed with the Structured Clinical Interview for DSM-IV Axis-II Disorders (SCID-II; First, Spitzer, Gibbon, \& Williams, 1994) were excluded. Additional exclusion criteria were acute suicide risk and inability to speak and read the Dutch language. Patients taking antidepressant medication were excluded, unless they were stable on medication for at least three months prior to the screening procedure. ${ }^{19}$

The study protocol was approved by the medical ethical committee of the University Hospital Maastricht, The Netherlands and the local ethics committee of the specialized secondary mental health facility where treatment was provided. All patients enrolled in the study signed written informed consent. Prior to patient recruitment, this study was registered (clinicaltrials.gov identifier: MASKED).

\section{Measures}

Beck Depression Inventory - second edition (BDI-II) - Depressive symptom severity was assessed with the BDI-II (Beck et al., 1996). The BDI-II assesses depressive symptom severity along 21-items with increasing severity ranging from 0 to 3 with higher scores representing more symptom severity. The Dutch version of the BDI-II was used in this study which had been shown to possess good psychometric properties (van der Does, 2002). The instructions of the original BDI-II were adapted in the current study, asking participants to evaluate depressive symptoms during the past week instead of the past two weeks.

Session Rating Scale (SRS) - The quality of the therapeutic alliance was assessed with the SRS (Duncan et al., 2003). The SRS is a brief, 4-item, instrument based on the definition of the therapeutic alliance by Bordin (1979) and measures the relational bond between client and therapist, goal agreement, and task agreement. An overall scale assesses whether the client feels that "there was something missing in the session" vs "overall the session was good for me" along a 100 mm. Visual Analogue Scale (VAS; Duncan et al., 2003). We used the overall scale in all analyses and additional analyses for the bond, goal and task subscales are reported. Participants completed the SRS weekly and the instructions were to rate the last treatment session. Satisfactory psychometric properties have been reported for the SRS (Duncan et al., 2003). To validate the SRS in the current study, we correlated the SRS subscales with the respective subscales from the Working Alliance Inventory (WAI) - Client Version short form (Tracey \& Kokotovic, 1989) that patients completed once a month. At the initial alliance assessment, the

\footnotetext{
${ }^{19}$ Medication dosage of one participant taking venlafaxine was increased from $75 \mathrm{mg}$ to $150 \mathrm{mg}$ two months prior to start of the study.
} 
subscales bond, goal, and task of the WAI correlated positively with the bond, goal, and task items of the SRS ( $r=.85 ; r=.62 ; r=.86$, respectively), demonstrating the construct validity of the SRS items.

Core-Belief Ratings (CBR) - After session 6 of the exploration phase, the therapist conducted a negative core-belief interview with the patient as described in McBride et al. (2007). Patients were asked questions about automatic thoughts, underlying assumptions, expectations, attitudes and early life experiences during a structured interview (McBride et al., 2007). The identified idiosyncratic core-beliefs for the five domains self, other, world, future, and interpersonal relationships were then phrased as a statement about the self, others, etc. For example, a possible core-belief about the self would be: "I am a failure". Based on the identified core-beliefs, 5 idiosyncratic VASs were constructed for each patient and included in the weekly assessment battery. Patients were asked to indicate the degree to which they agree with each statement on a $100 \mathrm{~mm}$ VAS. In particular, patients read the following instructions: "Next you will be presented with a number of statements about how people can think about themselves, others, the world, the future and interpersonal functioning. Please indicate for each statement in how far you agree with the statement by setting a mark on the line". Thus participants were not explicitly instructed to rate how much they agree with the statement right now ("state") and neither how much they agree with the statement in general ("trait"). The average of the 5 domains that were assessed was used in all analyses.

\section{Procedure}

Depressive symptoms, core-beliefs and the therapeutic alliance were assessed once a week from home, by logging on to a secured website. If patients did not complete the questionnaires within two days, a reminder email was sent. Completion rate of weekly questionnaires during the intervention phase was $72.5 \%$. Treatment sessions were scheduled independent of the weekly assessments. The ST intervention was divided into an exploration phase and an intervention phase. During the exploration phase schemas and symptoms were merely explored and no attempts were made to actively change schemas or resolve current problems. This phase served as an extra control phase to determine treatment effects. The intervention phase consisted of up to 65 individual sessions ST lasting $50 \mathrm{~min}$ each. In this phase, underlying schemas, emotional experiences and dysfunctional behaviors were targeted by the use of cognitive and experiential techniques, the therapeutic alliance (limited reparenting), and behavioral techniques (Young et al., 2003). Sessions were scheduled weekly initially and with lower frequency later in treatment. A more detailed description of the treatment manual of ST for cMDD is provided elsewhere (Renner, Arntz, et al., 2013). All analyses reported here are based on the intervention phase. 


\section{Analytical approach}

Due to the design of the study, different sequences of assessment - session combinations emerged between, as well as within, participants. In most instances weekly repeated assessments of depressive symptoms, core-beliefs, and the alliance of the previous session were separated by a treatment session. Thus, in these instances the participant completed an assessment some time before and some time after a therapy session. However, in other instances, for example when sessions were not scheduled weekly (e.g., due to illness or holidays), a treatment session was followed by a number of weekly assessments, resulting in repeated assessments of the therapeutic alliance of the same therapy session. Thus, in these instances the participant had a therapy session and completed at least two assessments on different days following the session. Given this data structure, we were able to test the three models of how change in core-beliefs could be related to change in depressive symptoms (illustrated and described in Figure 1) and the three models of how change in depressive symptoms could be related to the therapeutic alliance (illustrated and described in Figure 2).

To establish a timeline, we first computed the exact day of each session for each participant starting with day $=0$ for the first session in the intervention phase and the exact day of each assessment moment for each individual participant. For the assessment moments, the date on which a participant actually completed the assessment was used rather than the date on which the assessment was sent to the participant. When the assessment completion and the treatment session fell on the same date the exact time of the session and the exact time of the assessment were compared to determine whether the alliance rating referred to the same day session or to the previous session.

Next, each of the models described in Figure 1 and Figure 2 was computed per participant. Two distinct approaches were used to consolidate data from individual cases. The first approach involved computation of average weighted correlation coefficients. Individual correlation coefficients were first calculated per participant and transformed into Fisher's z-scores ${ }^{20}$. Next, individual z-scores were multiplied by the number of observations per participant, summed across participants and divided by the total number of observations in order to derive a weighted average. The weighted average z-score of each model was then back transformed to a correlation coefficient $r^{21}$. This approach gives a descriptive overview of the (temporal) correlation between the variables under study. By computing the correlation coefficients per individual it is assumed that each row in the correlation matrix represents independent observations. Yet, the repeated observation within individuals are not independent and likely autocorrelated. Moreover, it is unclear how a confidence interval for such an averaged coefficient should be determined.

\footnotetext{
${ }^{20}$ Fisher's $\mathrm{z}=0.5 * \operatorname{LN}((1+\mathrm{r}) /(1-\mathrm{r}))$

${ }^{21} \mathrm{r}=(\operatorname{EXP}(2 * \mathrm{z})-1) /(\operatorname{EXP}(2 * \mathrm{z})+1)$
} 


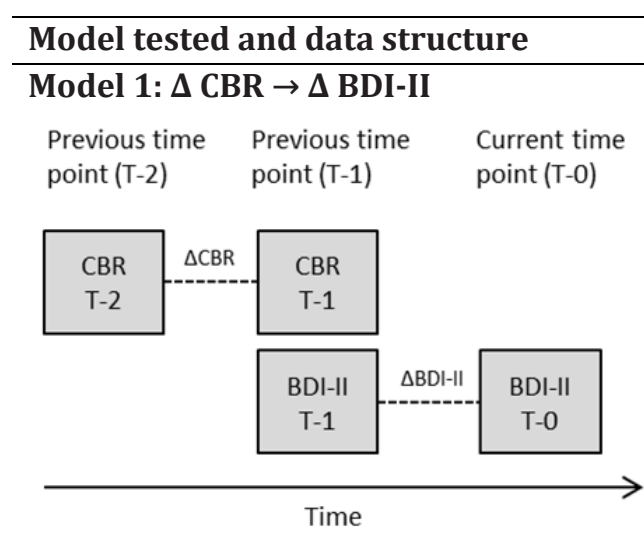

\section{Model 2: $\Delta$ BDI-II $\rightarrow \Delta \mathrm{CBR}$}

$\begin{array}{ll}\text { Previous time } & \text { Previous time } \\ \text { point }(\mathrm{T}-2) & \text { point }(\mathrm{T}-1)\end{array}$

Current time point (T-0)

\section{Description of model tested}

Change in core-beliefs predicts subsequent change in symptoms: Preceding change in core-beliefs $\left(\Delta \mathrm{CBR}_{\mathrm{T}}\right.$ 2,T-1) predicts subsequent change in depressive symptoms $\left(\Delta \mathrm{BDI}-\mathrm{II}_{\mathrm{T}-1, \mathrm{~T}-0}\right)$.

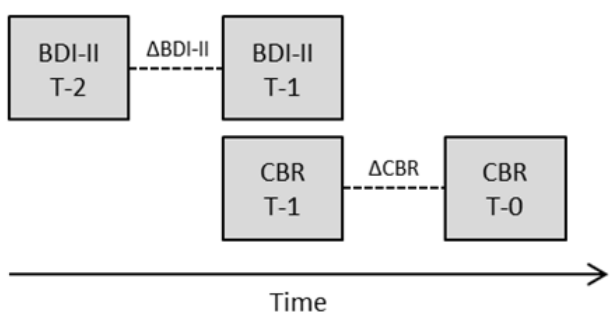

\section{Model 3: $\Delta$ BDI-II $\leftrightarrow \Delta$ CBR} Previous time
point (T-1)

Current time point (T-0)

BDI-II

$\mathrm{T}-0$

CBR

T-0
Change in symptoms predicts subsequent change in core-beliefs: Preceding change in depressive symptoms $\left(\Delta \mathrm{BDI}-\mathrm{II}_{\mathrm{T}-2,}, \mathrm{~T}-1\right)$ predicts subsequent change in core-beliefs $\left(\Delta \mathrm{CBR}_{\mathrm{T}-1, \mathrm{~T}-0}\right)$.

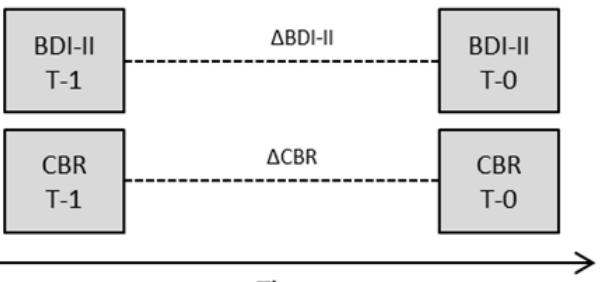

When symptoms change, core-beliefs change concurrently: Change in symptoms $\left(\Delta \mathrm{BDI}-\mathrm{II}_{\mathrm{T}-1, \mathrm{~T}-0)}\right.$ predicts concurrent change in core-beliefs $\left(\Delta C B R_{T}\right.$ $1, \mathrm{~T}-0)$.

Time

Figure 1. Three models testing the relation between negative core-beliefs and depressive symptoms. BDIII = Beck Depression Inventory second edition; CBR = Core-beliefs Ratings; $\mathrm{T}=$ Time. For model 1 and model 2 four different versions are possible depending on whether there was 1) a session between the core-belief assessments and between the BDI-II assessments; 2) no session between the core-beliefs assessment and a session between the BDI-II assessments; 3) a session between the core-belief assessments and no session between the BDI-II assessments; 4) no session between the core-belief assessments and no session between the BDI-II assessments. For model 3 two different version are possible, depending on whether there was 1) a session between the repeated assessments or 2) no session between the repeated assessments. 


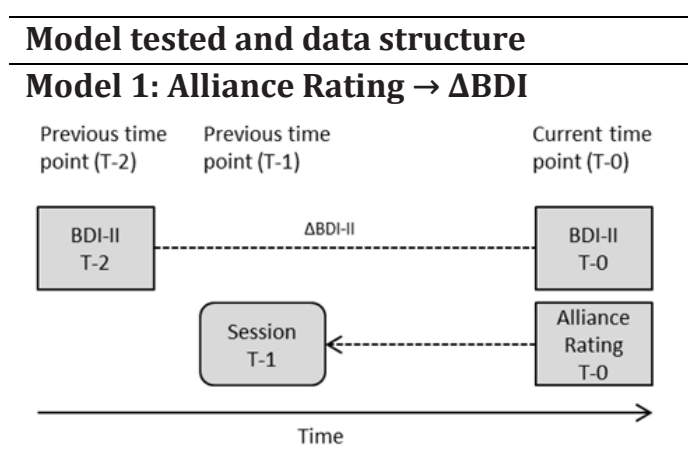

Note. The alliance rating at T- 0 reflects the rating of the last treatment session (T-1).

\section{Model 2: $\Delta \mathrm{BDI} \rightarrow$ Alliance Rating}

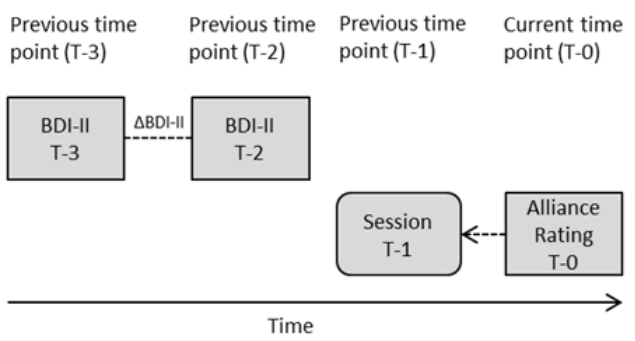

Note. The alliance rating at $\mathrm{T}-0$ reflects the rating of the last treatment session ( $\mathrm{T}-1)$.

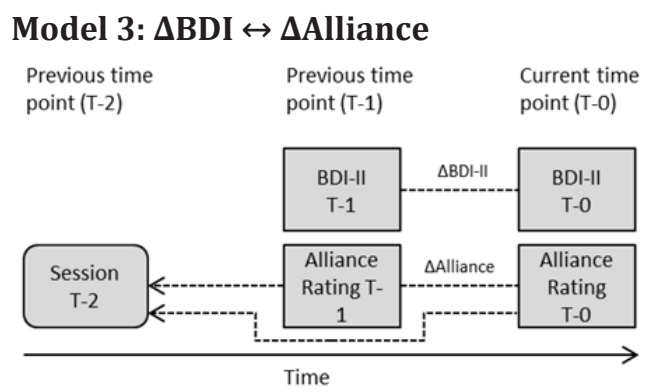

\section{Description of model tested}

Therapeutic alliance predicts symptom change: Change in depressive symptoms from presession (BDI-II T-2) to post-session (BDI-II T-0) is predicted by the therapeutic alliance rating (T- 0 ) of that session (T-1).
Symptom change predicts therapeutic alliance: The alliance rating (T-0) of the last session (T1 ) is predicted by change in depressive symptoms prior to the session ( $\triangle \mathrm{BDI} \mathrm{T}-3, \mathrm{~T}-2)$.
Symptoms and alliance change ratings change concurrently: Change in two subsequent alliance ratings $(\Delta$ Alliance Rating $T-1, T-0)$ of the same session (T-2) is concurrently related to change in depressive symptoms $(\Delta \mathrm{BDI}-\mathrm{II} \mathrm{T}-1$, $\mathrm{T}-0)$.

Note. Alliance ratings at T- 1 and T- 0 reflect ratings of the last treatment session (T-2). Therefore the arrows from the alliance ratings point backwards to the same session.

Figure 2. Three models testing the relation between the therapeutic alliance and depressive symptoms. BDI-II = Beck Depression Inventory second edition; $\mathrm{T}=$ Time . 
To derive an aggregated coefficient of the strength of the (temporal) relations between study variables as well as a test of statistical significance, the second approach involved a multi-level analysis (MLA). In the MLA, the participant was entered as subject variable, and an index variable, starting at 0 at the first assessment for each individual and then incrementing by 1 for each subsequent assessment, was entered as repeated variable. For each model an autoregressive (AR1) covariance structure was specified. The fixed part of each model included a main effect for the predictor variable, a main effect of time as well as time $\times$ predictor interactions. Additional fixed effects for time and time $\times$ predictor interactions were specified since the strength of the core-beliefs BDI-II relationship and the strength of the alliance - BDI-II relationship might change during treatment. The optimal representation of time, represented by the session number or by the day of assessment, was derived by comparing the model fit for linear and logarithmic representations of time. Backward deletion of non-significant predictors was used, starting with the interaction, to come to a final model. The dependent variable and fixed effects were specified per model as outlined below.

\section{Core-belief models}

Change scores for the BDI-II/CBR were calculated in a way that positive change scores reflected an increase in symptoms/core-beliefs. Average weighted correlation coefficients for the core-belief models were determined by entering scores for $\triangle \mathrm{CBR}$ and $\triangle \mathrm{BDI}-\mathrm{II}$ in a correlation matrix and by performing the computations outlined earlier. The strength of $\triangle \mathrm{CBR}$ and $\triangle \mathrm{BDI}$-II associations might differ in instances in which change in these variables occurs in the context of a therapy session (likely representing change due to the session) vs instances in which the change occurs in the absence of a therapy session (likely representing natural fluctuations). Therefore, separate analyses were performed for instances in which there was 1) a session between the CBR and between the BDI-II assessments; 2) no session between the CBR assessments and a session between the BDI-II assessments; 3) a session between the CBR assessments and no session between the BDI-II assessments; 4) no session between the CBR and the BDI-II assessments.

To compute core-belief model 1 , each instance in which two successive CBR assessments preceded two successive BDI-II assessments was considered (811 instances). In the MLA, $\triangle \mathrm{BDI}$-II was entered as dependent variable and $\triangle \mathrm{CBR}$, time and the time $\times \Delta$ CBR interaction were entered as fixed effects. The time $\times \Delta$ CBR interaction in these analyses reflects the degree to which the strength of the relation between change in the core-belief ratings and subsequent change in depressive symptoms differs during the course of treatment. The beta value of the main effect of $\Delta$ CBR reflects the estimated change in depressive symptoms with each preceding 1 point change in corebeliefs.

To compute core-belief model 2, each instance in which two successive BDI-II assessments preceded two successive CBR assessments was considered (813 instances). In the MLA, $\triangle \mathrm{CBR}$ was entered as dependent variable and $\triangle \mathrm{BDI}-\mathrm{II}$, time and the time $\times$ $\Delta \mathrm{BDI}-\mathrm{II}$ interaction were entered as fixed effect. The time $\times \Delta \mathrm{BDI}-\mathrm{II}$ interaction in these 
analyses reflects the degree to which the strength of the relation between change in the depressive symptoms and subsequent change in core-belief ratings differs during the course of treatment. The beta value of the main effect of $\Delta$ BDI-II reflects the estimated change in core-beliefs with each preceding 1 point change in depressive symptoms.

To compute core-belief model 3, each instance in which two BDI-II and two CBR assessments occurred concurrently was considered (885 instances). In the MLA, $\triangle \mathrm{CBR}$ was entered as dependent variable and $\Delta \mathrm{BDI}-\mathrm{II}$, time and time $\times \Delta \mathrm{BDI}-\mathrm{II}$ were entered as fixed effects. The time $\times$ BDI-II change score interaction in these analyses reflects the degree to which the strength of the relation between change in the depressive symptoms and concurrent change in core-belief ratings differs during the course of treatment. The beta value of the main effect of $\triangle B D I-I I$ reflects the estimated change in core-beliefs with each concurrent 1 point change in depressive symptoms. The choice to enter $\triangle \mathrm{CBR}$ as dependent variable in these analyses was purely conceptual and entering $\triangle \mathrm{BDI}$-II as dependent and $\triangle \mathrm{CBR}$ as independent variable in these analyses yields the same results.

\section{Alliance models}

Correlation coefficients for the alliance models were determined by constructing separate correlation matrices of: $\triangle B D I-I I$ (post-session - pre-session) and the alliance rating of the session (alliance model 1); $\Delta \mathrm{BDI}$-II prior to a session and the alliance rating of the subsequent session (alliance model 2); $\Delta \mathrm{BDI}$-II and concurrent change in the alliance rating of the same session assessed on separate days (alliance model 3). Correlations were then weighted and aggregated as outlined above. Change scores were calculated in a way that positive change scores in the BDI-II reflected an increase in symptoms and positive change scores of the alliance reflected more positive alliance ratings.

To compute alliance model 1, each instance in which a pre-session BDI-II and a post-session BDI-II as well as an alliance rating of the session between the two BDI-II assessments was available was considered (497 instances). In the MLA, $\triangle B D I-I I$ was entered as dependent variable and the alliance rating, time and the time $\times$ alliance interaction were specified as fixed effects. The time $\times$ alliance interaction reflects the degree to which the relation between the alliance and pre-session to post-session $\Delta \mathrm{BDI}$ II differs during the course of treatment. The beta value of the main effect of the alliance reflects the estimated $\triangle \mathrm{BDI}-\mathrm{II}$ score from one session to the next for each additional increase by 1 in alliance ratings of the session.

To compute alliance model 2, each instance in which two successive BDI-II assessments, with no session in between, occurred prior to a session was considered (222 instances). In the MLA, the general alliance rating was entered as dependent variable and $\triangle \mathrm{BDI}-\mathrm{II}$, time and the time $\times \Delta \mathrm{BDI}-\mathrm{II}$ interaction were specified as fixed effects. The time $\times \Delta \mathrm{BDI}-\mathrm{II}$ interaction reflects the degree to which the relation between $\triangle \mathrm{BDI}-\mathrm{II}$ before the session and the alliance rating of the session differs during the course of treatment. The beta value of the main effect of $\Delta$ BDI-II reflects the estimated alliance rating for each 1 point improvement on the BDI-II prior to the session. 
To compute alliance model 3, all instances in which two successive BDI-II assessments with concurrent alliance assessments and with no session in between, occurred following a session were considered (432 instances). In these instances the repeated therapeutic alliance rating reflected an alliance rating of the same session. In the MLA, $\Delta$ alliance was entered as dependent variable and $\Delta B D I-I I$, time and the time $\times$ $\Delta \mathrm{BDI}$-II interaction were entered as fixed effects. The time $\times \Delta \mathrm{BDI}-\mathrm{II}$ interaction reflects the degree to which the relation between $\Delta B D I-I I$ and $\Delta$ alliance rating of the same session differs during the course of treatment. The beta value of the main effect of $\triangle \mathrm{BDI}-$ II reflects the estimated concurrent $\Delta$ alliance ratings of the same session when the $\Delta \mathrm{BDI}-$ II increases by 1 point.

\section{Results}

Relation between change in core-beliefs and change in depressive symptoms

The average weighted and unweighted correlation coefficients for the three core-belief models are shown in Table 1. Results of the MLA are summarized below and in Table 2. For all MLA log-linear time provided the best fit and was used as representation of time.

Table 1. Average weighted and unweighted correlation coefficient for the core-belief models and for different assessment - session sequences

\begin{tabular}{llllll}
\hline Core-Belief Models & Overall & Sequence 1 & Sequence 2 & Sequence 3 & Sequence 4 \\
\hline $\begin{array}{l}\text { Model 1: weighted } r_{\Delta \mathrm{CBR} \rightarrow \triangle \mathrm{BDI}-\mathrm{II}} \\
\text { (unweighted } r \text { ) }\end{array}$ & $-.10(-.10)$ & $-.08(-.03)$ & $-.28(-.80)$ & $-.10(.69)$ & $.12(.43)$ \\
$\begin{array}{l}\text { Model 2: weighted } r_{\Delta \mathrm{BDI}-\mathrm{II} \rightarrow \triangle \mathrm{CBR}} \\
\text { (unweighted } r \text { ) }\end{array}$ & $.10(.19)$ & $-.03(-.73)$ & $-.09(-.41)$ & $-.05(-.54)$ & $.02(.32)$ \\
$\begin{array}{l}\text { Model 3: weighted } r_{\Delta \mathrm{BDI}-\mathrm{II}<->\Delta \mathrm{CBR}} \\
\text { (unweighted } r \text { ) }\end{array}$ & $.35(.34)$ & $.35(.35)$ & - & - & $.33(.26)$ \\
\hline
\end{tabular}

Note. Sequence 1-4 refers to the four possible assessment - session sequences: Sequence $1=$ only instances with a session between the CBR and BDI-II assessments were considered; Sequence $2=$ only instances with a session between the BDI-II assessments but not between the CBR assessments were considered; Sequence $3=$ only instances with a session between the CBR assessments but not between the BDI-II assessments were considered; Sequence $4=$ only instances in with no session between the CBR assessments and no session between the BDI-II assessments were considered.

Core-Belief Model 1 - Change in core-beliefs predicts subsequent change in symptoms The time $\times \triangle C B R$ interaction was not significant $(B=0.02, t(722.21)=1.41, p=.160)$ and therefore removed from the model. In the final model, the main effect of $\Delta C B R$ was not significant, $\mathrm{B}=0.01, t(800.81)=0.53, p=.600$. When only instances in which the BDI-II assessments but not the CBR assessments were separated by a session were considered, there was a significant main effect of $\triangle \mathrm{CBR}$ (after removing ns time interaction), $\mathrm{B}=$ $0.09, t(196.61)=-2.14, p=.034$, showing that improvements in core-beliefs prior to a session were associated with a worsening of depressive symptoms following the session. All other versions of assessment - session sequences revealed non-significant effects. 
Core-Belief Model 2 - Change in symptoms predicts subsequent change in core-beliefs The time $\times \Delta \mathrm{BDI}-\mathrm{II}$ interaction was not-significant $(\mathrm{B}=0.05, t(808.99)=1.32, p=.188)$, and therefore removed from the model. In the final model, the main effect of $\triangle B D I-I I$ was not significant, $\mathrm{B}=0.04, t(805.64)=0.70, p=.485$, showing that improvements in depressive symptoms did not predict subsequent improvements in core-beliefs. When only instances in which a session occurred between the BDI-II assessments but not between the CBR assessments were considered, the time $\times \triangle \mathrm{BDI}-\mathrm{II}$ interaction became significant, $\mathrm{B}=0.17, t(194.81)=2.31, p=.022$. To interpret the meaning of this interaction we divided the overall time variable into four equal phases and then plotted the fixed predicted $\triangle$ CBR scores, derived from the MLA, against $\triangle B D I-I I$ with separate lines for the four time phases, representing different stages of treatment (Figure 3). All other versions of assessment - session sequences revealed non-significant effects.

\section{Core-Belief Model 3 - Core-beliefs and depressive symptoms change concurrently}

The time $\times \Delta$ BDI-II interaction was not-significant $(\mathrm{B}=0.01, t(874.05)=0.15, p=.877)$, and therefore removed from the model. In the final model, the main effect of change in BDI-II was significant, $\mathrm{B}=0.66, t(873.65)=13.23, p<.001$, showing that improvements in depressive symptoms were concurrently associated with improvements in corebeliefs. When only instances in which a session occurred between the two assessments were considered, the main effect of change in BDI-II remained significant (after deletion of ns. interaction), $\mathrm{B}=0.60, t(478.10)=8.74, p=<.001$. When only instances in which no session occurred between the repeated assessments were considered, the time $x$ $\Delta \mathrm{BDI}-\mathrm{II}$ interaction became significant, $\mathrm{B}=-0.31, t(391.00)=-4.00, p=<.001$. To interpret the meaning of this interaction we plotted the fixed predicted $\triangle \mathrm{CBR}$ scores, derived from the MLA, against $\triangle$ BDI-II with separate lines for different phases of treatment (Figure 4). 
Table 2. Results of mixed regression analyses testing the relation between change in core-beliefs and change in depressive symptoms.

\begin{tabular}{|c|c|c|c|c|c|}
\hline & Beta & $S E$ & $D F$ & $t$ & $p$ \\
\hline \multicolumn{6}{|l|}{ Model 1} \\
\hline Intercept & 0.05 & 0.54 & 454.99 & 0.09 & .926 \\
\hline Time & -0.03 & 0.10 & 452.39 & -0.33 & .743 \\
\hline$\triangle \mathrm{CBR}$ & -0.09 & 0.08 & 746.10 & -1.18 & .238 \\
\hline Time $x \Delta C B R$ & 0.02 & 0.01 & 772.21 & 1.41 & .160 \\
\hline \multicolumn{6}{|c|}{ Model 1 after removing ns. interaction } \\
\hline Intercept & 0.09 & 0.54 & 450.92 & 0.17 & .862 \\
\hline Time & -0.04 & 0.10 & 448.08 & -0.51 & .676 \\
\hline$\Delta \mathrm{CBR}$ & 0.01 & 0.02 & 799.79 & 0.51 & .608 \\
\hline \multicolumn{6}{|l|}{ Model 1 final } \\
\hline Intercept & -0.13 & 0.13 & 377.74 & -1.00 & .318 \\
\hline$\triangle \mathrm{CBR}$ & 0.01 & 0.22 & 800.81 & 0.53 & .600 \\
\hline \multicolumn{6}{|l|}{ Model 2} \\
\hline Intercept & 0.14 & 0.84 & 489.93 & 0.17 & .867 \\
\hline Time & -0.06 & 0.16 & 486.93 & -0.36 & .721 \\
\hline$\Delta \mathrm{BDI}$ & -0.24 & 0.22 & 808.93 & -1.09 & .276 \\
\hline Time $\mathrm{x} \Delta \mathrm{BDI}$ & 0.05 & 0.04 & 808.99 & 1.32 & .188 \\
\hline \multicolumn{6}{|c|}{ Model 2 after removing ns. interaction } \\
\hline Intercept & 0.26 & 0.84 & 486.99 & 0.31 & .759 \\
\hline Time & -0.08 & 0.16 & 484.10 & -0.50 & 621 \\
\hline$\Delta$ BDI-II & 0.04 & 0.06 & 804.71 & 0.71 & .481 \\
\hline \multicolumn{6}{|l|}{ Model 2 final } \\
\hline Intercept & -0.15 & 0.19 & 417.49 & -0.75 & .451 \\
\hline$\Delta$ BDI-II & 0.04 & 0.06 & 805.64 & 0.70 & .485 \\
\hline \multicolumn{6}{|l|}{ Model 3} \\
\hline Intercept & 0.43 & 0.76 & 535.54 & 0.57 & .572 \\
\hline Time & -0.09 & 0.14 & 533.31 & -0.62 & .535 \\
\hline$\Delta \mathrm{BDI}$ & 0.63 & 0.21 & 871.10 & 3.01 & .003 \\
\hline Time $\mathrm{x} \Delta \mathrm{BDI}$ & 0.01 & 0.04 & 874.05 & 0.15 & .877 \\
\hline \multicolumn{6}{|c|}{ Model 3 after removing ns. interaction } \\
\hline Intercept & 0.44 & 0.75 & 537.28 & 0.58 & .561 \\
\hline Time & -0.09 & 0.14 & 534.92 & -0.64 & .524 \\
\hline$\Delta \mathrm{BDI}-\mathrm{II}$ & 0.66 & 0.05 & 872.48 & 13.23 & $<.001$ \\
\hline \multicolumn{6}{|l|}{ Model 3 final } \\
\hline Intercept & -0.03 & 0.17 & 468.04 & -0.17 & .863 \\
\hline$\Delta$ BDI-II & 0.66 & 0.05 & 873.65 & 13.23 & $<.001$ \\
\hline
\end{tabular}

Note. All time variables are log transformed 
15

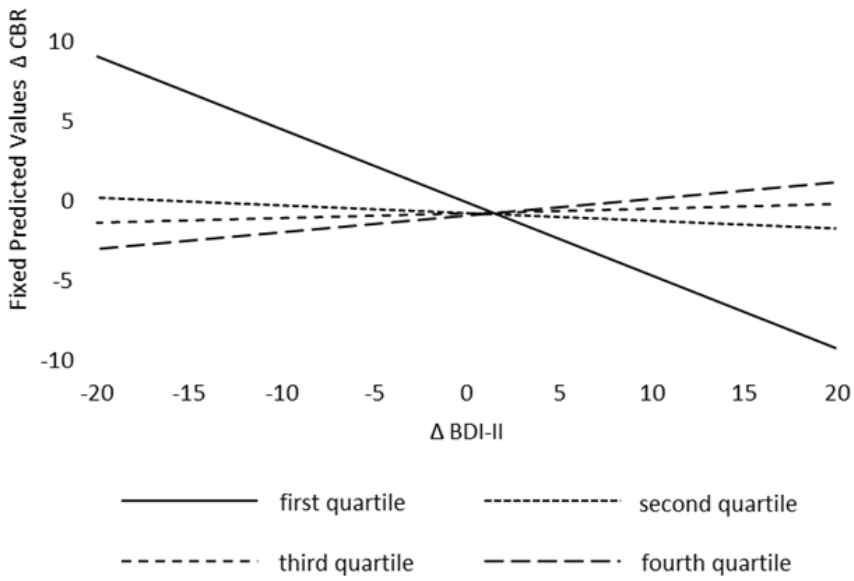

Figure 3. Illustration of the significant time $\times \Delta B D I-I I$ interaction predicting subsequent change in core beliefs in instances in which a session occurred between the BDI-II assessments but not between the CBR assessments. Positive change scores for the BDI-II reflect increased symptoms; Positive change scores for the CBR reflect an increase in negative core-beliefs. An increase in depressive symptoms from pre-session to post-session was associated with subsequent decrease in core-belief ratings in the absence of a session only in the early phase of treatment (first quartile). In later phases of treatment change in depressive symptoms was unrelated to subsequent change in core-beliefs.

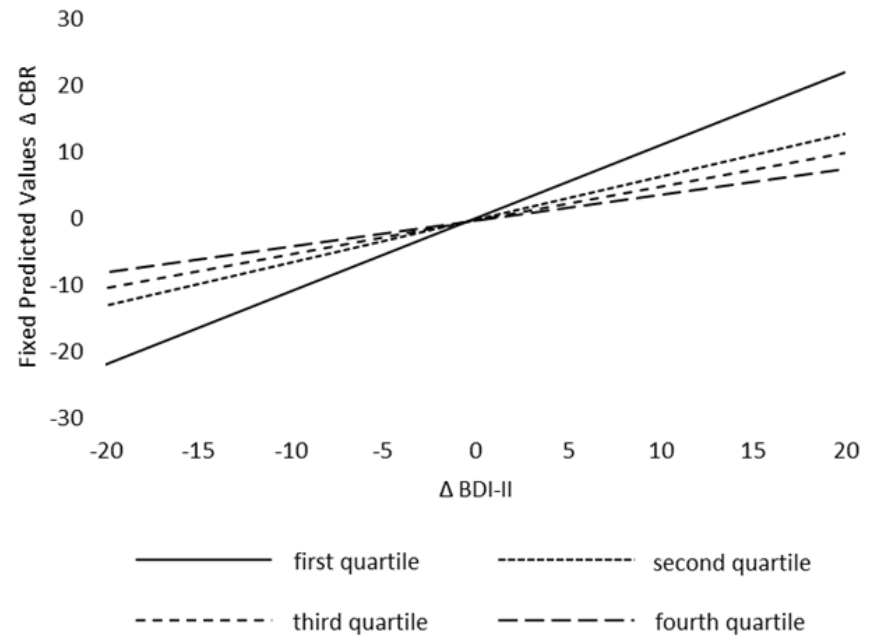

Figure 4. Illustration of the significant time $\times \Delta$ BDI-II interaction predicting concurrent change in core beliefs in instances in which no session occurred between the concurrent BDI-II and CBR assessments. Positive change scores for the BDI-II reflect increased symptoms; Positive change scores for the CBR reflect an increase in negative core-beliefs. An increase in depressive symptoms was associated with an increase in negative core-beliefs and the strength of this association became gradually weaker during the course of treatment. 


\section{Relation between therapeutic alliance and depressive symptoms}

The average weighted and unweighted correlation coefficient for the three alliance models are summarized in Table 3. For all MLA the log-linear time variable provided the best fit to the data and was therefore used as representation of time. Model 1 and 2 were conducted with the log transformed session number as representation of time and with the log transformed day of assessment as additional sensitivity analyses. Model 3 was conducted with the log transformed day of assessment as representation of time because assessments for this model could also occur in the absence of a session. The results of the MLA of the alliance models are summarized below and in Table 4. All analyses were repeated separately for the subscales bond, goal and task (not reported in Table 4).

Table 3. Average weighted and unweighted correlation coefficient for the alliance models

\begin{tabular}{lllll}
\hline Alliance Models & Overall & Bond & Goal & Task \\
\hline Model 1: weighted $r_{\text {Alliance }->\Delta \text { BDI-II }}($ unweighted $r)$ & $.02(-.15)$ & $.05(-.07)$ & $.12(.11$ & $.07(-.01)$ \\
Model 2: weighted $r_{\Delta \mathrm{BDI}-\mathrm{III} \rightarrow \text { Alliance }}$ (unweighted $\left.r\right)$ & $-.05(-.06)$ & $-.05(-.30)$ & $.01(-.05)$ & $.01(.18)$ \\
Model 3: weighted $r_{\Delta \mathrm{BDI}-\mathrm{II}<>\text { Alliance }}$ (unweighted $\left.r\right)$ & $-.02(.22)$ & $-.08(-.46)$ & $-.15(-.58)$ & $-.08(-.04)$ \\
\hline
\end{tabular}

\section{Alliance Model 1 - Change in depressive symptoms is predicted by alliance}

The time $\times$ overall alliance interaction was not significant $(\mathrm{B}=0.01, t(303.70)=1.04, p$ $=.30$ ) and therefore removed from the model. In the final model, overall alliance was not significant, $\mathrm{B}=0.01, t(261.59)=0.64, p=.524$, showing that change in depressive symptoms from pre-session to post-session was not predicted by the therapeutic alliance of that session. Similarly, separate analyses with the bond, goal, and task subscales revealed non-significant effects. Additional sensitivity analyses included MLAs with the log-transformed day of assessment as time variable and analyses in which only pre-session assessments were considered that occurred two days or less before the session because it could be argued that it is more likely that the session causes change in symptom severity from pre-session to post-session if the session occurs relatively shortly after the initial pre-session BDI-II assessment. These sensitivity analyses resulted in comparable results.

\section{Alliance Model 2 - Change in depressive symptoms predict alliance ratings}

The time $\times \Delta \mathrm{BDI}-\mathrm{II}$ interaction was not significant $(\mathrm{B}=0.15, t(185.32)=0.91, p=.364)$ and therefore removed from the model. In the final model, $\triangle B D I-I I$ was not significant, $B$ $=0.05, t(162.55)=0.41, p=.684$, showing that changes in depressive symptoms prior to a session did not affect alliance ratings of the session. Similarly, separate analyses with the bond, goal, and task subscales revealed non-significant effects as did sensitivity analyses with the log-transformed day of assessment as time variable. 
Alliance Model 3 - Two subsequent alliance ratings of the same session change with changes in mood state

The time $\times \Delta \mathrm{BDI}-\mathrm{II}$ interaction was not significant $(\mathrm{B}=-0.08, t(424.78)=-0.95, p=.343)$ and therefore removed from the model. After removing the ns interaction there was a significant main effect of time, $\mathrm{B}=0.67, t(262.95)=2.05, p=.04$, showing that in situations in which the therapeutic alliance of the same session was evaluated twice, on separate days, the second evaluation was more negative and that this effect was more profound in early phases of treatment but leveled out throughout treatment. In the final model, $\Delta$ BDI-II was not significant, $\mathrm{B}=0.02, t(418.45)=0.20, p=.844$, showing that changes in the overall alliance rating of the same session did not vary with concurrent changes in depressive symptoms.

In the model including the bond subscale change score as dependent variable, the time $\times \Delta \mathrm{BDI}-\mathrm{II}$ interaction was not significant, $\mathrm{B}=-0.11, t(425.82)=-1.27, p=.21$, and therefore removed from the model. After removing the ns interaction there was a significant main effect of time $B=0.75, t(268.31)=2.22, p=.027$, showing that bond ratings increased over time. In the final model, the main effect of bond was not significant, $B=-0.14, t(421.68)=-1.31, p=.190$. In the model including the goal subscale change score as dependent variable, the time $\times \Delta \mathrm{BDI}-\mathrm{II}$ interaction was significant, $\mathrm{B}=-$ $0.30, t(424.64)=-3.00, p=.003$. Similarly, in the model including the task subscale change score as dependent variable, the time $\times \Delta$ BDI-II interaction was significant, $\mathrm{B}=-$ $024, t(427.60)=-2.73, p=.007$. To interpret the meaning of these interaction terms, we plotted the predicted change in the goal and task subscales, derived from the MLA, against change in BDI-II scores with separate lines representing different phases of treatment (Figure 5). To test the robustness of these findings, we conducted sensitivity analyses in which only instances were included in which two assessments moments occurred after a session because these alliance ratings might be more accurate. In these sensitivity analyses weighted concurrent correlations and results of MLA were comparable to those of the initial analyses. 
Table 4. Results of mixed regression analyses testing the relation between the therapeutic alliance and changes in depressive symptoms.

\begin{tabular}{|c|c|c|c|c|c|}
\hline & Beta & $S E$ & DF & $\boldsymbol{t}$ & $p$ \\
\hline \multicolumn{6}{|l|}{ Model 1} \\
\hline Intercept & 1.81 & 3.02 & 285.06 & 0.60 & .548 \\
\hline Time & -0.02 & 0.03 & 283.74 & -0.66 & .512 \\
\hline Overall alliance & -1.36 & 1.16 & 305.11 & -1.17 & .243 \\
\hline Time $\mathrm{x}$ Overall alliance & 0.01 & 0.01 & 303.70 & 1.04 & .301 \\
\hline \multicolumn{6}{|c|}{ Model 1 after removing ns. interaction } \\
\hline Intercept & -1.05 & 1.21 & 258.34 & -0.87 & .385 \\
\hline Time & 0.01 & 0.01 & 262.29 & 0.78 & .436 \\
\hline Overall alliance & -0.17 & 0.19 & 260.30 & -0.91 & .362 \\
\hline \multicolumn{6}{|l|}{ Model 1 final } \\
\hline Intercept & -1.35 & 1.16 & 261.33 & -1.16 & .246 \\
\hline Overall alliance & 0.01 & 0.01 & 261.59 & 0.64 & .524 \\
\hline \multicolumn{6}{|l|}{ Model 2} \\
\hline Intercept & 80.32 & 4.57 & 107.74 & 17.56 & $<.001$ \\
\hline Time & 2.19 & 1.45 & 108.65 & 1.51 & .134 \\
\hline$\Delta$ BDI-II & -0.43 & 0.55 & 188.90 & -0.79 & .430 \\
\hline Time x $\Delta$ BDI-II & 0.15 & 0.17 & 185.32 & 0.91 & .364 \\
\hline \multicolumn{6}{|c|}{ Model 2 after removing ns. interaction } \\
\hline Intercept & 79.91 & 4.55 & 106.08 & 17.55 & $<.001$ \\
\hline Time & 2.31 & 1.44 & 107.23 & 1.60 & .113 \\
\hline$\Delta \mathrm{BDI}-\mathrm{II}$ & 0.05 & 0.13 & 161.61 & 0.40 & .687 \\
\hline \multicolumn{6}{|l|}{ Model 2 final } \\
\hline Intercept & 86.60 & 1.83 & 33.50 & 47.25 & $<.001$ \\
\hline$\Delta$ BDI-II & 0.05 & 0.13 & 162.55 & 0.41 & .684 \\
\hline \multicolumn{6}{|l|}{ Model 3} \\
\hline Intercept & -5.51 & 1.80 & 265.90 & -3.06 & .002 \\
\hline Time & 0.68 & 0.33 & 260.54 & 2.07 & .039 \\
\hline$\Delta \mathrm{BDI}-\mathrm{II}$ & 0.48 & 0.48 & 425.07 & 0.99 & .322 \\
\hline Time $x \Delta$ BDI-II & -0.08 & 0.09 & 424.78 & -0.95 & .343 \\
\hline \multicolumn{6}{|c|}{ Model 3 after removing ns. interaction } \\
\hline Intercept & -5.48 & 1.81 & 267.97 & -3.03 & .003 \\
\hline Time & 0.67 & 0.33 & 262.95 & 2.05 & .041 \\
\hline$\Delta \mathrm{BDI}-\mathrm{II}$ & 0.03 & 0.10 & 418.85 & 0.25 & .799 \\
\hline \multicolumn{6}{|l|}{ Model 3 final } \\
\hline Intercept & -1.89 & 0.46 & 162.82 & -4.09 & $<.001$ \\
\hline$\Delta$ BDI-II & 0.02 & 0.10 & 418.45 & 0.20 & .844 \\
\hline
\end{tabular}

Note. All time variables are log transformed 


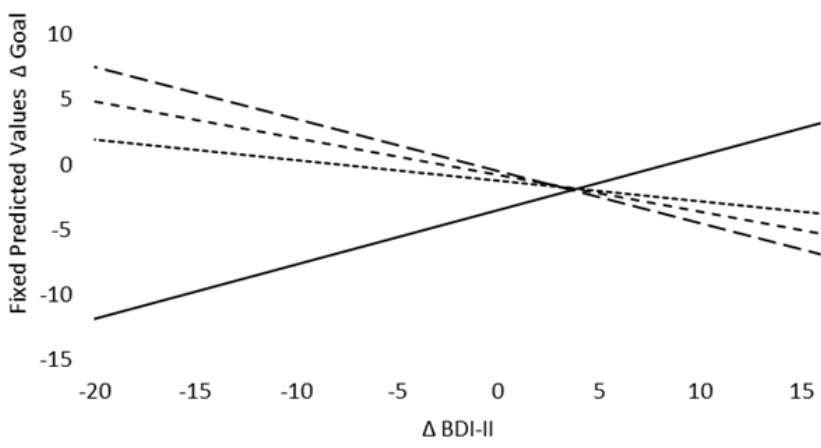

10

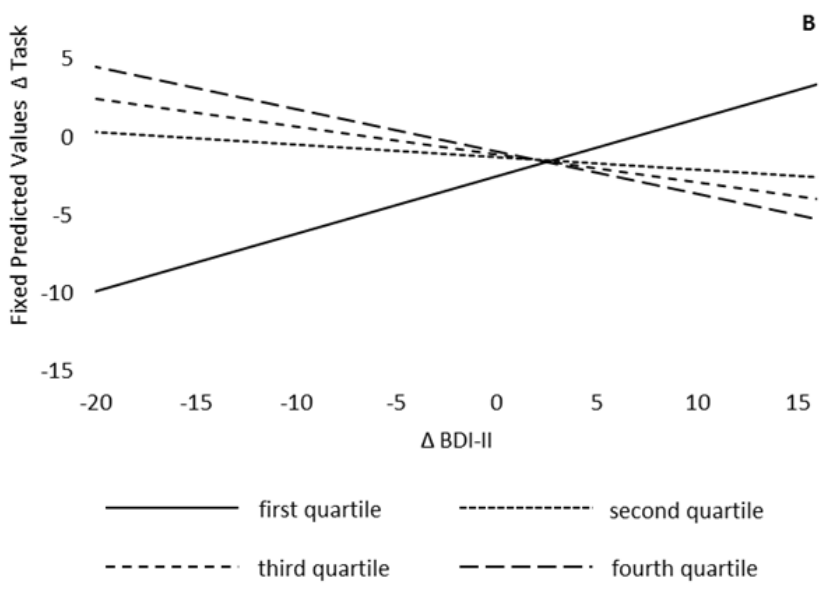

Figure 5. Illustration of the significant time $\times \Delta$ BDI-II interactions predicting concurrent change in goal agreement (panel A) and task agreement (panel B). In general, repeated goal and task agreement ratings of the same session became more positive in situations in which depressive symptoms decreased concurrently, whereas goal and task agreement ratings of the same therapy session became more negative in situations in which depressive symptoms increased concurrently (dashed lines). In the first quartile of treatment this general pattern was reversed, such that repeated goal and task agreement ratings of the same session became more negative in situations in which depressive symptoms decreased concurrently and more positive in situations in which depressive symptoms increased concurrently (solid lines). 


\section{Discussion}

The aim of this study was to test potential mechanisms of symptom change in schema therapy (ST) for chronic major depressive disorder (cMDD). In particular, we focused on two potential key mechanisms of symptom change in ST, schemas (as proxied by negative idiosyncratic core-beliefs) and the therapeutic alliance. An innovative approach to study the temporal relation among these variables was applied, focusing on the within-person variance in these variables, i.e. differences in changes in these variables over time within participants. We proposed and tested six models of how negative corebeliefs and the therapeutic alliance might be related to changes in depressive symptom severity during ST for cMDD (Figure 1 and Figure 2).

Changes in negative core-beliefs did not predict subsequent changes in depressive symptoms (core-belief model 1) as theory would suggest (Young et al., 2003). The only exception was found in instances in which changes in core-beliefs occurred in the absence of a therapy session and subsequent changes in depressive symptoms occurred following a therapy session. In these instances, improvements in core-beliefs prior to a session were associated with a worsening of symptoms following the session. It should be noted that this effect was rather small. Each improvement in core-beliefs by 1 point (on a scale from 0-100) was associated with an estimated subsequent increase in depressive symptoms, as assessed by the BDI-II, by 0.09 points.

Changes in depressive symptoms did not predict subsequent changes in negative core beliefs (core-belief model 2). However, when only instances in which a session occurred between the assessments of depressive symptoms but not between the assessments of core-beliefs were considered, there was a significant interaction of changes in depressive symptoms with time. In these instances, an increase in depressive symptoms was associated with a decrease in negative core-beliefs only early on in treatment. Later on in treatment changes in depressive symptoms were unrelated to changes in negative core beliefs.

Analyses of the concurrent core-belief model (core-belief model 3) suggest that changes in negative core-beliefs go together with concurrent changes in depressive symptoms. In other words, when symptoms improve core-beliefs improve concurrently. In general, this association remains stable throughout treatment, however in instances in which concurrent changes in these variables are evaluated in the absence of therapy sessions the association became weaker throughout treatment.

In general our findings regarding the core-belief models suggest that changes in negative core-beliefs do not precede (core-belief model 1) or follow (core-belief model 2) from changes in depressive symptom severity. Only in instances in which change in depressive symptoms occurred in the context of a therapy session and change in negative core-beliefs occurred in absence of a therapy session, change in these two variables was temporarily associated. One explanation for these findings could be that changes in core-beliefs in the absence of a therapy session do not represent structural changes in the underlying core-beliefs but rather naturalistic mood-fluctuations. In instances in which depressive symptoms improved prior to a session it might be more 
likely that mood partly regresses back at a subsequent assessment, contributing to the negative association. Similarly, in instances in which core-belief ratings become more positive in the absence of a therapy session, likely reflecting mood fluctuations rather than structural changes, the mood state might swing back in subsequent ratings, which would explain the negative association with changes in depressive symptom severity. In situations in which core-beliefs ratings occurred in the context of a therapy session, change in core-beliefs more likely reflects structural changes and in these instances no temporal association with changes in depressive symptoms was found. This explanation is highly speculative and other explanations for the observed findings are possible. The most robust evidence was found for a concurrent relation between changes in corebeliefs and changes in depressive symptoms. One explanation for this finding could be that both measures might partly assess the same overall symptomatic distress factor. Another possibility is that improvement in both variables is driven by another, unknown process. Yet another explanation could be that the timing of assessments did not capture the timing in which changes in these variables took place and that an even more fine grained temporal analyses of processes is necessary to disentangle the temporal relations between these variables. For example, it is possible that change in negativecore beliefs drives subsequent change in symptoms on the following day. With the current frequency of weekly assessments this would be erroneously reflected in a concurrent relation rather than a temporal relation between these variables. More fine grained analyses of temporality with repeated assessments every day (e.g. experience sampling) might shed more light on these process outcome relations, however practically it might be difficult to use such methodology over a very long treatment duration such as in ST for cMDD.

Another potential mechanism that might explain symptom change in ST for cMDD that was tested in the current study is the therapeutic alliance. In general, we found no evidence that the therapeutic alliance of a session was related to change in depressive symptoms from pre-session to post-session (alliance model 1). Thus our findings suggest that the alliance does not predict symptom change, when the temporal relation between alliance ratings and assessments of depressive symptoms is taken into account. Research synthesis on the alliance - outcome relation have reported small to moderate positive associations between alliance ratings and treatment outcome (Horvath et al., 2011). However, these previous studies primarily relied on cross-sectional research designs, focusing on differences in alliance ratings between subjects when predicting treatment outcome rather than changes in alliance ratings within-subjects over time. Typically, in these studies between subject differences in alliance ratings assessed at the beginning of treatment are related to between subject differences in treatment outcomes. In the current analyses, repeated alliance ratings within-individuals were related to session-to-session changes. If the alliance would be related to better outcomes than this should be reflected in positive associations between session-to-session changes and alliance ratings of the session (alliance model 1) but this was not supported in the current study. Moreover, we found no evidence for the second alliance model, 
stating that changes in depressive symptoms prior to a session would predict the subsequent alliance rating of the session.

The most robust evidence in the current study was found for the model predicting a concurrent association of changes in alliance ratings of the same therapy sessions and concurrent changes in depressive symptoms (alliance model 3). Findings of the current study suggest that changes in depressive symptoms are concurrently related to changes in the goal and task subscales of the alliance and that the strength of this association varies over the course of treatment. In particular, we found that repeated goal and task agreement assessments of the same session became more negative in situations in which depressive symptoms increased concurrently, indicating that alliance assessments are partly colored by the current level of depressive symptoms. Moreover, in an early treatment phase, this pattern was reversed showing that improvements in depressive symptoms were associated with more negative ratings of the goal and task agreement. One explanation for this finding might be that early on in treatment patients do not attribute improvements in symptoms to the specific techniques used in treatment whereas later on in treatment improved symptoms might be more readily attributed to the specific techniques (task agreement) used in treatment, resulting in the positive associations found in this study.

The findings of the current study should be interpreted in the light of several limitations. First, due to the design of this study the sample size was relatively small. It should be noted, however, that due to the frequent repeated assessments and the long duration of treatment a relatively large amount of data was available to test the proposed models. Second, in the current study we tested relatively straightforward models of how core-beliefs and the therapeutic alliance could be related to change in depressive symptoms. However, it is possible that the interrelationships between these variables are more complex but due to the relatively small sample size we were not able to test more complex multivariate models. Third, by design the current study did not include a control group and we were therefore not able to conduct formal mediation analyses. Fourth, we relied entirely on self-report measures to assess the variables under study, however, by assessing idiosyncratic core-beliefs we were able to assess only those core-beliefs that were particularly relevant for each individual client. Fifth, an important limitation is the way we assessed schemas. Ratings of explicit negative corebeliefs might not tap the schema change processes that underlie ST. It is possible, for example, that changes in schemas first occur on more implicit levels, or in emotions before they are expressed in cognitions. It is possible, that our core-belief proxyassessment of schemas tapped cognitive, rather than emotional aspects of schemas. Similarly, it is possible, that initially a growth in positive beliefs occurs before a reduction in negative beliefs takes part. Yet another possibility would be that the change process starts with doubt in core-beliefs instead of a change in core-belief. The appropriate assessment during treatment of cognitive constructs hypothesized to underlie changes in symptoms remains a challenging issue.

In conclusion, the current study advances previous process outcome studies by introducing an innovative approach to study process - outcome relations. Focusing on 
temporal relations between changes in negative core-beliefs and changes in depressive symptoms, we found mixed evidence for temporal relations. Based on the current analyses we can only conclude that changes in negative core-beliefs improve when depressive symptoms improve. Regarding the temporal precedence between the therapeutic alliance and symptom improvements, we found no evidence that change in symptoms was related to alliance ratings. However, alliance ratings seem to be at least in part colored by changes in current mood state. Disentangling the temporal relations between change in symptom levels and potential common factors of change (e.g. therapeutic alliance) and treatment specific factors of symptom change (e.g. schemas) remains an important challenge for future research that is far from resolved. 


\section{References}

Bamelis, L. L. M., Bloo, J., Bernstein, D., \& Arntz, A. (2012). Effectivness studies. In M. F. Van Vreeswijk, J. Broersen \& M. Nadort (Eds.), The Wiley-Blackwell handbook of schema therapy: theory, research and practice (pp. 495-510). Chichester: Wiley-Blackwell.

Beck, A. T., Steer, R. A., \& Brown, G. K. (1996). Manual for the Beck Depression Inventory-II. San Antonio, TX.

Bordin, E. (1979). The generalizability of the psychoanalytic concept of the working alliance. Psychotherapy(16), 252-260.

Carter, J. D., McIntosh, V. V., Jordan, J., Porter, R. J., Frampton, C. M., \& Joyce, P. R. (2013). Psychotherapy for depression: a randomized clinical trial comparing schema therapy and cognitive behavior therapy. $J$ Affect Disord, 151(2), 500-505.

Cuijpers, P., van Straten, A., Schuurmans, J., van Oppen, P., Hollon, S. D., \& Andersson, G. (2010). Psychotherapy for chronic major depression and dysthymia: A meta-analysis. Clinical Psychology Review, 30(1), 51-62.

DeRubeis, R. J., Brotman, M. A., \& Gibbons, C. J. (2005). A Conceptual and Methodological Analysis of the Nonspecifics Argument. Clinical Psychology: Science and Practice, 12(2), 174-183.

Duncan, B. L., Miller, S. D., Sparks, J. A., Reynolds, L. R., Brown, J., \& Johnson, L. D. (2003). The Session Rating Scale: Preliminary Psychometric Properties of a "Working" Alliance Measure. Journal of Brief Therapy, 3(1), 3-12.

First, M. B., Spitzer, R. L., Gibbon, M., \& Williams, J. B. (1997). Structured Clinical Interview for DSM-IV Axis I Disorders (SCID-I). New York: Biometrics Research Department.

First, M. B., Spitzer, R. L., Gibbon, M., \& Williams, J. B. W. (1994). Structured Clinical Interview for DSM-IV Axis II personality disorders (SCID-II). New York: New York State Psychiatric Institute, Biometrics Research Department.

Hoffart, A., Oktedalen, T., Langkaas, T. F., \& Wampold, B. E. (2013). Alliance and outcome in varying imagery procedures for PTSD: a study of within-person processes.J Couns Psychol, 60(4), 471-482.

Horvath, A. O., Del Re, A. C., Fluckiger, C., \& Symonds, D. (2011). Alliance in individual psychotherapy. Psychotherapy (Chic), 48(1), 9-16.

Kazdin, A. E. (2007). Mediators and mechanisms of change in psychotherapy research. Annual Review of Clinical Psychology, 3, 1-27.

Kraemer, H., Wilson, G., Fairburn, C. G., \& Agras, W. (2002). Mediators and moderators of treatment effects in randomized clinical trials. Archives of General Psychiatry, 59(10), 877-883.

Malogiannis, I. A., Arntz, A., Spiropoulou, A., Tsartsara, E., Aggeli, A., Karveli, S., et al. (2014). Schema Therapy for patients with chronic depression: A single case series study. Journal of Behavior Therapy and Experimental Psychiatry, 45(3), 319-329.

McBride, C., Farvolden, P., \& Swallow, S. R. (2007). Major Depressive Disorder and Cognitive Schemas. In L. P. Riso, P. L. Du Toit, D. J. Stein \& J. E. Young (Eds.), Cognitive Schemas and Core Beliefs in Psychological Problems. A Scientist-Practitioner Guide. Washington: American Psychological Association.

Nordahl, H. M., Holthe, H., \& Haugum, J. A. (2005). Early Maladaptive Schemas in Patients with or without Personality Disorders: Does Schema Modification Predict Symptomatic Relief? Clinical Psychology \& Psychotherapy, 12(2), 142-149.

Renner, F., Arntz, A., Leeuw, I., \& Huibers, M. (2013). Treatment for chronic depression using schema therapy. Clinical Psychology Science and Practice, 20, 166-180.

Renner, F., Arntz, A., Peeters, F., Lobbestael, J., \& Huibers, M. (2014). Schema therapy for chronic depression: Results of a multiple single case series study. Submitted manuscript.

Renner, F., van Goor, M., Huibers, M., Arntz, A., Butz, B., \& Bernstein, D. (2013). Short-term group schema cognitive-behavioral therapy for young adults with personality disorders and personality disorder features: Associations with changes in symptomatic distress, schemas, schema modes and coping styles. Behaviour Research and Therapy, 51(8), 487-492. 
Spijker, J., de Graaf, R., Bijl, R. V., Beekman, A. T., Ormel, J., \& Nolen, W. A. (2002). Duration of major depressive episodes in the general population: results from The Netherlands Mental Health Survey and Incidence Study (NEMESIS). Br J Psychiatry, 181, 208-213.

Spijker, J., van Straten, A., Bockting, C. L. H., Meeuwissen, J. A. C., \& van Balkom, A. J. L. M. (2013). Psychotherapy, antidepressants, and their combination for chronic major depressive disorder: A systematic review. The Canadian Journal of Psychiatry / La Revue canadienne de psychiatrie, 58(7), 386-392.

Spinhoven, P., Giesen-Bloo, J., van Dyck, R., Kooiman, K., \& Arntz, A. (2007). The therapeutic alliance in schema-focused therapy and transference-focused psychotherapy for borderline personality disorder. J Consult Clin Psychol, 75(1), 104-115.

Tracey, T. J., \& Kokotovic, A. M. (1989). Factor structure of the Working Alliance Inventory. Psychological Assessment: A Journal of Consulting and Clinical Psychology, 1(3), 207-210.

van der Does, A. J. W. (2002). Handleiding: De Nederlandse versie van de Beck Depression Inventory, 2 de Editie. [The Dutch version of the Beck Depression Inventory, 2nd ed.]. Lisse: Swets and Zeitlinger b.v.

Whiteford, H. A., Degenhardt, L., Rehm, J., Baxter, A. J., Ferrari, A. J., Erskine, H. E., et al. (2013). Global burden of disease attributable to mental and substance use disorders: findings from the Global Burden of Disease Study 2010. The Lancet, 382(9904), 1575-1586.

Young, J. E. (2006). Young Schema Questionnaire-3. New York: Cognitive Therapy Center.

Young, J. E., Klosko, J., \& Weishaar, M. E. (2003). Schema therapy: A practitioner's guide. New York: Guilford Press. 


\section{CHAPTER 11 \\ General Discussion}


CHAPTER 11 
The primary aim of this dissertation was to test the effects of schema therapy for chronic depression in a single case-series and to explore underlying mechanisms of change in schema therapy for chronic depression. The secondary aim of this dissertation was to examine underlying neurological and psychological vulnerability factors to (chronic) depression, as well as the stability of psychological vulnerability factors throughout treatment. To address these aims, part I of this dissertation describes the results of two functional magnetic resonance imaging (fMRI) studies that were conducted in the same sample of patients with chronic depression and non-patient controls. In the first study the neural correlates of self-referential processing in patients with chronic depression and in healthy matched controls were determined (chapter 2). In the second study, the default mode network functional connectivity during rest was investigated in patients with chronic depression and healthy matched controls, before and after a sad mood induction procedure (chapter 3). Part II describes the results of four individual studies: one study on the stability of early maladaptive schemas during outpatient treatment for depression (chapter 4), one study on the two-year stability of the personality traits neuroticism and extraversion in treated and untreated persons with depression (chapter 5), one study testing the stability of interpersonal problems during cognitive therapy for depression (chapter 6) and a meta-analysis quantifying the effects of psychotherapy for depression on psychosocial functioning (chapter 7). In the third part of this dissertation the theoretical application of schema therapy to treat patients with chronic depression is discussed (chapter 8) and the results of a single case-series testing the effects of schema therapy for chronic depression are presented (chapter 9). The temporal relation of change in depressive symptoms and two potential underlying mechanisms of change, the therapeutic alliance and negative core-beliefs, in schema therapy for chronic depression are explored in chapter 10.

In the current chapter a summary and critical discussion of the studies described in the previous chapters of this dissertation is presented. Methodological considerations relevant to this dissertation are also addressed in this chapter. Finally, implications of the results of this dissertation for clinical practice and future research are presented and the chapter ends with an overall conclusion.

\section{Summary of main findings}

\section{Part I - Neuroimaging studies in chronic depression}

In chapter 2 the neural correlates of self-referential processing in chronic depression and associations between neural activity in brain areas associated with self-referential processing and explicit and implicit measures of self-associations were investigated in order to shed light on the neural underpinnings of processing of negative self-relevant information in chronic depression. Patients with chronic depression and non-patient controls were presented with words that were either positive, neutral, general negative, depression related negative or non-words during functional Magnetic Resonance 
Imaging (fMRI). Participants were instructed to press a button whenever they detect a non-word. Compared to non-patient controls, patients with chronic depression had relatively decreased activity in the medial Prefrontal Cortex during processing of negative depression related vs. neutral words. Non-patient controls had relatively increased dorsolateral Prefrontal Cortex activity during processing of positive vs. neutral words whereas patients with chronic depression had relatively decreased activity. Explicit but not implicit measures of self-associations were correlated to brain activity in these areas. Based on these findings we concluded that, patients with chronic depression show, on the neural level, an emotional blunting response towards emotional stimuli.

In chapter 3 the effects of a sad mood induction procedure on the default mode network resting state connectivity in the same sample of patients with chronic depression and matched non-patient controls was investigated using fMRI. Patients with chronic depression and non-patient controls underwent a 6-min fMRI scan while resting quietly, before and after a sad mood induction procedure. The sad mood induction procedure consisted of mood suggestive music in combination with autobiographical recall of a sad personal event. Based on a posterior cingulate cortex (PCC) seed, a functional connectivity analysis of the two resting-state measurements was conducted. PCC resting state connectivity with the left parahippocampal gyrus, the left superior temporal gyrus and the left anterior inferior temporal cortex increased in patients with chronic depression and decreased in non-patient controls following the sad mood induction. PCC connectivity with the left anterior prefrontal cortex decreased in patients with chronic depression and increased in non-patient controls, following the sad mood induction. PCC connectivity with the right precuneus decreased slightly in patients with chronic depression but not in non-patient controls, following the sad mood induction. We interpreted these findings as evidence of general increased default mode network functional connectivity in patients with chronic depression following a sad mood induction and argue that this increased connectivity might reflect the activation of negative latent schemas and/or decreased control over ruminative thoughts about sad experiences, triggered by the mood induction in patients with chronic depression. The finding of increased PCC - left anterior prefrontal cortex connectivity following the mood induction in non-patient controls was interpreted as evidence for a cognitive control mechanism preventing negative memory recall in non-patient controls, following the sad mood induction.

\section{Part II - Stability of underlying risk-factors for depression}

In part II of this dissertation it was tested if underlying psychological vulnerability factors to depression improve during treatment for depression. In chapter 4, we sought to study the relation between early maladaptive schemas and depressive symptom severity, as well as the stability of early maladaptive schemas during a course of outpatient treatment for depression. The sample consisted of 132 depressed outpatients 
treated at a specialized mood disorder mental health care facility. Depressive symptom severity and early maladaptive schemas were assessed before and 16 weeks after starting with treatment. In multivariate linear regression analyses, the early maladaptive schemas emotional deprivation, abandonment and instability and failure were concurrently related to depressive symptom severity. Overall, patients with depression improved on most schemas from pre-treatment to post-treatment, but the effect sizes were rather small, whereas the effect size for improvements in depressive symptoms during treatment were moderate to large. Moreover, relative stability (regression) coefficients of schemas suggested that scores on the schema questionnaire remained relatively stable during the course of treatment, even after controlling for depressive symptom severity. Based on these findings we concluded that the underlying vulnerability in patients with depression, in terms of early maladaptive schemas, at least in part remains intact following outpatient treatment for depression.

The personality traits neuroticism and extraversion (more precisely the opposite, introversion) might also represent part of the underlying vulnerability to depression. In chapter 5, the two-year stability of neuroticism and extraversion in 709 treated and untreated persons with depression, participating in the Netherlands Study of Depression and Anxiety was investigated. The personality trait neuroticism decreased over a period of two years in persons with depression who sought treatment and in persons with depression who did not seek treatment during the study period. Depressive symptoms also improved in all participants and these improvements were highly associated with improvements in neuroticism. Extraversion did not change during the study period. These findings show that changes in neuroticism during treatment likely represent mood-state effects, rather than structural changes attributable to treatment.

The overall aim of chapter $\mathbf{6}$ was to study the stability of interpersonal problems over a course of 16-20 session individual cognitive therapy for depression, in order to determine if cognitive therapy has positive effects on interpersonal functioning. Moreover, we sought to explore associations between a patient's interpersonal style, the therapeutic alliance and improvements in depressive symptoms. The sample consisted of 523 outpatients with recurrent major depressive disorder, participating in a multistage two center study (Jarrett \& Thase, 2010). Using a principal components analysis, we identified three orthogonal factors of the inventory of interpersonal problems that were labeled general distress, agency and communion. The general distress factor decreased over a course of cognitive therapy whereas the agency and communion factors remained stable. These findings show that interpersonal functioning can be conceptualized in terms of more stable, trait aspects (interpersonal style) and less stable state aspects (interpersonal distress). Whereas the state aspects improve during cognitive therapy for depression the interpersonal style of patients does not change during treatment with cognitive therapy. For example, patients with a predominantly submissive interpersonal style before treatment report to tend to remain predominantly 
submissive in interpersonal situations after treatment but generally experience less distress.

Interpersonal functioning can be seen as a specific aspect of the broader concept of social functioning. In this sense, chapter 7 extended the scope of chapter $\mathbf{6}$ by quantifying the effects of various forms of psychotherapy on the broader concept of social functioning in a meta-analysis. We included 31 published randomized controlled trials with 2956 patients that were either assigned to a psychotherapy condition or a control group. Relative to control groups, psychotherapy had small to moderate positive effects on the social functioning of patients. Moreover, the treatment format and the number of sessions significantly predicted the effect size of psychotherapy for social functioning, with studies using individual treatment formats and studies with more treatment sessions resulting in higher effect sizes. These positive effects were partly independent from the effects of psychotherapy on depressive symptom severity. The results of this study show that the positive effects of psychotherapy for depression on depressive symptom severity extend to improvements in social functioning.

\section{Part III -Schema therapy for chronic depression}

In chapter 8, the theoretical application of schema therapy as a novel psychological treatment for chronic depression was described. We summarize the role of four underlying vulnerability factors to chronic depression, namely early adversity, cognitive factors, personality pathology and interpersonal factors. My co-authors and I describe how these factors can be addressed in schema therapy for chronic depression and a global treatment protocol of schema therapy for chronic depression is provided.

Chapter 9 describes the results of a single case-series testing the effects of schema therapy for chronic depression. The study consisted of three phases, a $6-24$ weeks baseline phase during which no treatment took place, a 12 week exploration phase, during which the underlying schemas were explored and related to current problems, and an intervention phase during which participants received up to 65 sessions of individual schema therapy for chronic depression. The primary outcome measure was depressive symptom severity assessed with the Beck Depression Inventory - second edition and the Quick Inventory of Depressive Symptomatology. Secondary outcome measures were global symptomatic distress, assessed with the Brief Symptom Inventory and overall functioning, assessed with the Outcome Rating Scale. Categorical outcomes included response, defined as a $50 \%$ reduction in depressive symptoms, remission, defined as a score of 6 or smaller on the Quick Inventory of Depressive Symptomatology, and recovery, defined as the absence of a depression diagnosis at post treatment according to the DSM-IV criteria, assessed with the SCID-I interview. At the end of the study period, eight patients were still in treatment and of these patients no posttreatment SCID interviews were available. Relative to the baseline phase and exploration phase of the study, there was a significant decrease in depressive symptoms 
and general symptomatic distress whereas overall functioning improved during the intervention phase. Depressive symptoms and global symptomatic distress initially also improved during the baseline phase but this effect leveled out in later weeks of the baseline phase. Effect sizes for improvements in depressive symptom severity, global symptomatic distress and overall functioning during the intervention phase were moderate to large. Following schema therapy, $40 \%$ of the patients responded to treatment, $35 \%$ remitted and 58\% recovered. Recovery rates are based on a subsample of 12 patients for whom diagnostic status at post-treatment was assessed. The findings from this study suggest that schema therapy is a potentially effective treatment for patients with chronic depression.

In chapter 10 the temporal relation between change in depressive symptoms and two potential mechanisms of change (negative core-beliefs and therapeutic alliance) in schema therapy for chronic depression were explored. In particular, six models of how change in depressive symptoms might be related to changes in negative core-beliefs and the therapeutic alliance were tested. The first three models tested whether 1) change in core-beliefs drives subsequent change in symptoms; 2) change in symptoms drives subsequent change in core-beliefs; or 3) whether core-beliefs and symptoms change concurrently (i.e. at the same time). The remaining three models tested whether 4) change in symptoms from pre-session to post-session is predicted by the alliance rating of that session; 5) change in depressive symptoms prior to a session predicts the alliance rating of that session; and 6) whether repeated assessments of the therapeutic alliance of the same session concurrently changes with changes in symptoms. Change in negative core-beliefs was concurrently related to changes in depressive symptoms, showing that core-beliefs improved together with improvement in symptoms. Change in core-beliefs and change in depressive symptoms were not temporarily related, showing that change in core-beliefs did not drive subsequent change in depressive symptoms and that change in depressive symptoms did not drive change in core-beliefs. Moreover, change in depressive symptoms was unrelated to the therapeutic alliance but alliance ratings were at least in part colored by changes in the current mood state: In general, in situations in which the goal and task agreement of the same session was evaluated twice, on separate days, the second evaluation was more positive in situations in which depressive symptoms decreased concurrently, whereas task and goal agreement ratings of the same therapy session became more negative in situations in which depressive symptoms increased concurrently. However, in the early phase of treatment (first quartile), task and goal agreement ratings of the same session became more negative in situations in which depressive symptoms decreased and more positive in situations in which depressive symptoms increased. From the findings of this study we concluded that, based on our current data, theoretical models of how change in core-beliefs is related to changes in symptoms are not supported. Moreover, the hypothesis that change in depressive symptoms is temporarily related to the therapeutic alliance was not supported. 


\section{Critical discussion of the main findings}

The results of chapter 2 suggest that patients with chronic depression show, on a neural level, an emotionally blunted reaction in brain areas associated with self-referential processing (medial prefrontal cortex) when presented with emotional stimuli, especially negative depression related words. Such blunted emotional responses have previously been associated with lower remission rates from depression (Peeters, Berkhof, Rottenberg, \& Nicolson, 2010). Clinically, these findings suggest that patients with chronic depression might benefit from treatment that specifically focuses on emotional processing. Findings from chapter 3 suggest that patients with chronic depression show increased resting-state functional connectivity following a sad mood induction in brain areas that have previously been suggested to be involved in schematic and ruminative processing (Disner, Beevers, Haigh, \& Beck, 2011; Marchetti, Koster, Sonuga-Barke, \& De Raedt, 2012). In contrast, non-patient controls show a relative decrease in connectivity within these brain areas. It is possible that the decreased connectivity following the sad mood-induction reflects an attempt of non-patient controls to not activate negative schemas that could be challenged by the mood induction and increased attempts to control ruminative tendencies that might have been activated during the moodinduction (note that thinking about a sad personal event was part of the mood induction instruction).

Chapters 4 to 7 showed that a range of underlying psychological vulnerability factors to depression improved during treatment for depression. On the one hand, this indicates that the effects of current treatments for depression extend to other areas of functioning and psychological wellbeing, such as psychosocial functioning (chapter 7). On the other hand, the improvements in these variables were usually smaller than the improvements in depressive symptoms, suggesting that part of the underlying vulnerability to depression remains after treatment. Since current treatments for depression have a strong focus on reduction in depressive symptom severity, rather than a focus on, for example, changing personality, this finding is not surprising. Thus, overall the findings from chapters 4 to 7 indicate that part of the underlying vulnerability to depression remains intact, following treatment, even if depressive symptoms improve. Although speculative this might explain, at least in part, the high relapse and recurrences found in depressed patients.

Early maladaptive schemas are one of the potential vulnerability factors to (chronic) depression that have been studied in this dissertation. The relation between specific sets of early maladaptive schemas and depressive symptoms that were found in chapter $\mathbf{4}$ are largely in line with the previous literature investigating such associations (Calvete, Estévez, López de Arroyabe, \& Ruiz, 2005; Harris \& Curtin, 2002). When the stability of early maladaptive schemas was assessed over the course of 16-weeks of outpatient treatment, we found that early maladaptive schemas remained relatively stable and that changes in the mean level from pre- to post-treatment were rather small. This finding is in line with two previous studies showing that early maladaptive schemas remain relatively stable over time (Riso et al., 2006; Wang, Halvorsen, Eisemann, \& 
Waterloo, 2010). However, this finding extends previous findings by showing that early maladaptive schemas remain stable even in the context of treatment for depression, indicating that the underlying vulnerability, in terms of early maladaptive schemas, remains stable during treatment for depression. Given that some early maladaptive schemas in this study were specifically related to depressive symptom severity, it is possible that patients with depression might benefit from treatment that aims to decrease the impact of these early maladaptive schemas, such as schema therapy.

The personality factors neuroticisms and extraversion have also often been discussed as two other potential vulnerability factors to (chronic) depression. Chapter $\mathbf{5}$ investigated the stability of neuroticism and extraversion, over a period of two years. We found that changes in these personality traits were largely driven by changes in the underlying mood state, rather than by treatment per se. This finding highlights an important methodological issue namely that, depending on the measurement instruments used, the assessment of personality variables can be strongly dependent on the current mood state (Karsten et al., 2012). While our study suggest that neuroticism changes with the current mood state a more fine grained analyses of the temporal relation between these personality traits and depressive symptoms might lead to different conclusions. In our study extraversion and neuroticism was only assessed at two time points and it therefore was impossible to disentangle temporal relations between changes in these variables and changes in depressive symptoms.

In chapter 6 we showed that interpersonal problems, as assessed with the inventory of interpersonal problems (Horowitz, Rosenberg, Baer, Ureno, \& Villasenor, 1988), can be represented in terms of three underlying factors that describe state aspects of interpersonal functioning (interpersonal distress) as well as trait aspects (interpersonal style). In line with a previous study on the stability of these three factors during cognitive therapy for depression (Vittengl, Clark, \& Jarrett, 2003), we found that general interpersonal distress decreased during treatment, whereas the interpersonal style of patients remained stable. Thus, patients who started treatment with a general socially avoidant interpersonal style, for example, tended to retain this general style of behavior in interpersonal situations following treatment with cognitive therapy. However, the distress that was experienced in interpersonal situations was decreased after cognitive therapy for depression. In terms of targeting the underlying vulnerability to depression it might be important to also achieve change in the interpersonal style during treatment for depression. Although speculative, the non-assertive and socially avoidant interpersonal style of depressed patients might contribute to relapse and recurrences if no alternative interpersonal behaviors (e.g. assertiveness) are learned in treatment.

In chapter 7 we tested the impact of psychotherapy for depression on psychosocial functioning in a meta-analysis. Compared to a control group, psychotherapy resulted in significant improvements in psychosocial functioning with moderate effect sizes. The effects of psychotherapy on social functioning remained significant after controlling for the effects on depressive symptom severity. This indicates that improvements in psychosocial functioning were not fully accounted for by 
improved depressive symptoms alone but that treatment also had positive effects on psychosocial functioning in addition to depressive symptoms. In multivariate metaregression analyses we found that the number of treatment sessions was related to the effect size of psychotherapy on social functioning, with studies that had included more treatment sessions resulting in higher effects. This suggests that more treatment sessions might be needed to repair the impaired psychosocial functioning in patients with depression. A previous meta-analysis has shown that patients with chronic depression also benefit from more treatment sessions in terms of symptomatic improvements (Cuijpers et al., 2010). Together, these findings highlight the importance of longer treatment durations in (chronic) depression in order to reach higher improvements on a symptomatic level and in terms of improved psychosocial functioning.

In general, the findings from part I and part II of this dissertation suggest that patients with (chronic) depression might benefit from a more integrative treatment approach that takes the underlying psychological vulnerability factors to depression into account, such as schema therapy. In schema therapy a specific focus is given to break through the blunted emotional processing found in chronic depression (chapter 2) and the ultimate goal of schema therapy is to decrease the impact of negative latent schemas that might more readily be triggered by sad-mood in patients with chronic depression (chapter 3 ). Moreover, schema therapy is an integrative treatment approach that makes use of several techniques from different psychotherapeutic orientations to treat the various underlying risk-factors to depression, such as early maladaptive schemas (chapter 4), personality (chapter 5), and interpersonal and social functioning (chapters 6 and 7).

Chapter 8 provides a theoretical model of how the underlying psychological vulnerability factors to chronic depression can be addressed in schema therapy. Based on a review of the underlying vulnerability factors, it is suggested that schema therapy could be an effective treatment for patients with chronic depression. To test this central claim of this chapter, we conducted a single case-series study of schema therapy for chronic depression (chapter 9). Findings from this study suggest that schema therapy has positive effects on depressive symptom severity, global symptomatic distress, and overall functioning, when compared to a baseline or exploration phase period. Although the findings from this study cannot be directly compared to findings from randomized controlled trials or meta-analyses, it is informative to benchmark the effect sizes and response and remission rates found in the study against those of previous studies. Table 1 provides an overview of the main outcomes from chapter 9 and compares these findings with effect sizes and response and remission rates found in previous studies quantifying or testing the effects of psychotherapy for chronic depression. 
Table 1. Benchmarking the effects of the study described in chapter 9 with effects sizes and response and remission rates previously reported for psychotherapy for chronic depression.

\begin{tabular}{llll}
\hline Study & Type of Study & Psychotherapy & Main outcomes of interest for benchmarking \\
\hline Chapter 9 & Single case series & Schema therapy & Remission: $35 \%$ \\
& & Response: $40 \%$ \\
& & & Effect size (for improvement from last baseline to last \\
& & & intervention phase assessment): $d$ between 0.91 and \\
& & 1.30 \\
Cuijpers & Meta-analysis & Various & Mean effect size for contrast psychotherapy vs. control \\
et al. 2010 & & & group: $d=0.23$
\end{tabular}

Spijker et al. Systematic review Various 2013

$\begin{array}{lll}\begin{array}{l}\text { Keller et al. } \\ 2000\end{array} & \text { RCT } & \text { CBASP } \\ \begin{array}{l}\text { Wiersma et } \\ \text { al. in press }\end{array} & \text { RCT } & \text { CBASP }\end{array}$

al. in press

Malogiannis Single case series et al. 2014
Mean effect size for improvement from baseline to post-test: $d=1.16$

Of three studies comparing psychotherapy for chronic major depressive disorder to a placebo condition, one resulted in an effect size of 0 , one in a negative effect size and one in a moderate positive effect size. None of the contrasts between psychotherapy and placebo were significant.

Within the CBASP group, completers (ITT):

Remission: 24\% (33\%)

Overall response (response or remission): 52\% (48\%)

Within the CBASP group, completers (ITT):

Remission: 26\% (19.4\%)

Response: $41.2 \%$ (31.3\%)

Effect size (within CBASP group): $d=1.37$

Schema therapy Remission: $41.6 \%$

Overall response (response or remission): $58.2 \%$

Effect size (for improvements from baseline to posttest): $d$ between 2.01 and 2.84

The overview of previous studies in Table 1 is not exhaustive. Studies in Table 1 were selected so that the comparison includes a meta-analysis/systematic review (Cuijpers et al., 2010; Spijker, van Straten, Bockting, Meeuwissen, \& van Balkom, 2013), two major randomized clinical trials (Keller et al., 2000; Wiersma et al., in press) and a comparable single case-series testing the effects of schema therapy for chronic depression (Malogiannis et al., 2014). When benchmarking the effect sizes of the study described in chapter 9 with those reported in the meta-analysis by Cuijpers et al. (2010), schema therapy seems to perform above average. Response and remission rates of the study described in chapter 9 are roughly in line with those found for the cognitive behavioral analysis system of psychotherapy (CBASP; Keller et al., 2000; Wiersma et al., in press), a form of psychotherapy specifically developed for those with chronic depression. Moreover, response and remission rates of the current study are comparable with those of a recent single-case-series of schema therapy in a smaller sample of chronically depressed women (Malogiannis et al., 2014). It should be noted that the results of a single case-series cannot be directly compared to those of a randomized clinical trial or 
findings from meta-analyses. However, in the study described in chapter 9, stringent control phases were used to control for a general decrease in symptoms over time (baseline phase) and to control for possible effects of receiving empathy and attention from the therapist (exploration phase). These phases resemble those of a waiting-list control condition (baseline phase) and of a non-active control treatment (exploration phase) in a RCT. Overall, our case-series study adds to the emerging body of evidence suggesting that schema therapy might be an effective treatment for chronic depression, at least as effective as current treatment options for chronic depression and potentially more effective, but more controlled and comparative treatment outcome studies, comparing schema therapy to other active treatments (e.g. treatment as usual) are needed to further build the evidence base of schema therapy for chronic depression.

In chapter 10 the temporal relation between change in depressive symptoms during schema therapy and two potential mechanisms of change (negative core beliefs and the therapeutic alliance) in schema therapy for chronic depression were explored. We used an innovative approach to study the temporal relation between these variables, focusing on the within-person variance (i.e. changes in these variables within persons over time) rather than focusing on the between-person variance (i.e. differences in these variables between participants). By mapping out the temporal relations between these variables we were able to test six models on the temporal and concurrent relations between change in depressive symptoms, negative core-beliefs and the therapeutic alliance. We found that an increase in depressive symptoms from pre-session to postsession was associated with a subsequent decrease in negative core-beliefs but only when change in symptoms but not change in core-beliefs occurred in the context of as session and only in the first quartile of treatment. One explanation for this finding could be that change in core-beliefs in the absence of a therapy session does not reflect structural changes in underlying beliefs but rather mood-state fluctuations and that it is more likely that mood-state returns to a baseline after a previous improvement. However, this explanation remains speculative and cannot be tested within this study. In line with the idea that fluctuations in core-beliefs in the absence of therapy session represent mood-state fluctuations, rather than structural changes, it was found that change in depressive symptoms predicted concurrent change (i.e. change at the same time) in core-beliefs but only in instances in which change in both variables occurred in the absence of therapy sessions. The finding that, in general (i.e. when ignoring assessment-session sequences), change in core-beliefs was not temporarily related to change in depressive symptoms was contrary to what would be expected based on theory. There are at least two possible explanations for this finding: 1) the theory is wrong and other mechanisms are responsible for changes in symptoms; 2) our approach to study temporal relations did not capture the critical change moments during treatment. While it would be too premature to accept the first explanation based on the current data, it is probably most fruitful to further explore innovative methods to study potential mechanisms of symptom change in the future.

The second potential mechanism of symptom change in schema therapy for chronic depression that was explored in this study was the therapeutic alliance. When 
alliance ratings of the same therapy sessions were assessed repeatedly, specific aspects of the alliance (goal and task agreement) became more positive when depressive symptoms improved. However, taking different treatment phases into account revealed that in the first quartile of treatment these ratings became more negative when symptoms improved. One potential explanation for this finding could be that early on in treatment patients do not attribute improvements in symptoms to the specific techniques (task agreement) that were used. Again based on the current study this explanation remains speculative and alternative explanations cannot be ruled out. The finding that the overall therapeutic alliance ratings are not temporarily related to symptom changes was in contrast to previous findings that have reported moderate alliance outcome relations (Horvath, Del Re, Fluckiger, \& Symonds, 2011). However, most previous studies were based on cross-sectional associations whereas the current study took the temporal relations between change in symptoms and alliance ratings from session to session into account. Based on our analyses, again at least two possible conclusions regarding the alliance - symptom change association can be drawn. 1) the therapeutic alliance in schema therapy for chronic depression is not temporarily related to changes in depressive symptoms and other mechanisms are responsible for changes in symptoms; 2) our approach to study alliance - symptom change relations did not capture the critical change moments during treatment. The finding that some aspects of the therapeutic alliance (goal and task agreement) were concurrently related to changes in depressive symptoms suggest that alliance assessments are in part colored by the current mood state of participants. In this context a fruitful avenue for future research might be the development of alternative alliance measures that are less affected by the current mood state. It might be interesting, for example, to explore possibilities of developing implicit measures of the therapeutic alliance.

Overall, based on the studies conducted for this dissertation two important critical aspects should be mentioned. First, (chronic) depression entails more than just depressive symptoms and additional outcomes should be considered when investigating treatment effects. For example, from a patient perspective, social functioning might be an important treatment outcome that is currently not included in definitions of treatment response or remission (chapter 7). Second, instead of evaluating treatment outcomes, a stronger focus on treatment processes that lead to these outcomes is needed. To do so, it is necessary to assess treatment outcomes and potential mechanisms that lead to these outcomes repeatedly throughout treatment and to evaluate the temporal relations between these variables (chapter 10). However, even when a large number of repeated assessments is available, such that the temporal relations between potential mechanisms and symptoms can be established, other issues, such as the exact timing of assessments or the measurement and operationalization of concepts, complicate the study of treatment processes that lead to outcomes. 


\section{Methodological considerations}

\section{Methodological shortcomings}

A number of important methodological shortcomings that apply to the studies in this dissertation should be mentioned. Since we relied on a variety of research designs in the studies presented in this dissertation (experimental neuroimaging, observational designs, cohort study, meta-analysis, single case-series) there is no single, overarching, methodological issue that applies to all the studies described in this dissertation. In general, it can be stated that each study in the current dissertation had its own methodological strengths and weaknesses that had mainly to do with the research design of each individual study.

In the neuroimaging studies described in part I of this dissertation (chapters 2 and 3) we did not include a clinical control group. It therefore remains unclear how specific the results are with respect to chronic depression. It is possible that comparable results would have been obtained in patients with non-chronic forms of depression or related mental disorders. The issue of lack of specificity is a limitation that applies to most previous neuroimaging studies in depression on which the studies described in part I of this dissertation were based. Thus one general advice would be that neuroimaging studies in psychiatric patients with a given mental disorder should move beyond demonstrating differences between patients and healthy participants. By including patients with comparable psychiatric and treatment history background, yet distinct mental disorder the specificity of the underlying neuropathology of a given mental disorder could be established. In the context of depression, depending on the specific research questions, appropriate clinical control conditions might be patients with primary anxiety disorders or patients with cluster-c personality disorders because of overlap in some aspects of symptomatology.

The studies described in part II of this dissertation each have their specific methodological shortcomings that have to do with the different research designs that were used in the respective studies. Chapter 4 describes the findings from a naturalistic treatment study. In such study designs a number of factors that might impact the results of the study are not controlled for, such as the quality and amount of treatment, as well as patient characteristics such as co-morbidity. On the other hand, naturalistic treatment studies are less affected by problems that have to do with selection-bias and findings from these studies might therefore more readily generalize to everyday practice.

Chapter 5 describes the findings from a cohort study in which patients with depression were followed over a period of two years. In this study the stability of personality traits in treated and untreated patients were explored. One major methodological issue in this study was that patients were not-randomized to treatments and the group of patients that received treatment might therefore not be comparable to the group of patients that did not receive treatment. The results of this study should therefore not be interpreted in terms of effects of treatment on personality traits but 
rather in terms of the stability of personality traits in people with depression who seek treatment compared to people with depression who do not seek treatment.

The study in chapter $\mathbf{6}$ evaluated the effects of cognitive therapy for depression on interpersonal problems. The conclusions that can be drawn from this study are limited by the fact that the acute-phase of this multi-phase study only included a single treatment condition (cognitive therapy) and no control condition. It therefore remains unclear how specific the findings are with respect to cognitive therapy. It is possible, for example, that psychotherapy with a stronger focus on interpersonal functioning (e.g. interpersonal psychotherapy) has stronger effects on interpersonal problems in depression.

Chapter 7 describes the results of a meta-analysis that quantifies the effect of psychotherapy for depression on social functioning. Meta-analyses in general are a rather crude way of exploring treatment effects and are not always well suited to answer specific questions about predictors, mediators or moderators of treatment effects. Although we were able to include various potential predictors in multivariate meta-regression analyses it is difficult to disentangle the relation between study characteristics and the effect-size of interest. For example, we found no differences between different types of psychotherapy on the effect size of social functioning. However this finding is based on different sets of comparisons rather than direct comparison between different types of psychotherapy within the same study, which limits the conclusions that can be drawn from such findings. Methodologically, metaanalyses could be improved by obtaining study-level data and basing the analyses on the original study-level data rather than group statistics reported in publications.

In chapter 8 the theoretical application of schema therapy to chronic depression is described. The aim of this chapter was to describe the rationale of applying schema therapy to chronic depression. In doing so we did not conduct a formal systematic literature review and therefore only describe a selected number of studies to support our central claims. Moreover, the model that we describe in chapter 8 should be regarded as a working model with a limited scope on psychological factors. Chronic depression is a complex disorder and other psychological factors (e.g. attachment) and biological factors (e.g. genes, neurotransmitters, hormones) are also important to consider when describing underlying vulnerability to chronic depression. However, in our model we aimed to focus on specific psychological factors that can be addressed in schema therapy for chronic depression.

In chapter 9 we describe the results of a single case-series testing the effects of schema therapy for chronic depression. The single case-series design does by definition not include a control group which limits the conclusions that can be drawn from the study. Although we found positive effects of schema therapy on depressive symptom severity, it remains unclear how schema therapy would have performed relative to another active treatment condition. We relied on comparing treatment effects with symptom changes during a baseline period, however, symptoms also changed during the baseline period which complicated the interpretation of the data in this study substantially. More specifically, it is unclear what have caused the change in symptoms 
during the baseline period. One possibility would be that patients with chronic depression indeed become less symptomatic when repeatedly completing questionnaires, possibly because they gain insights into their symptoms. However, given that symptoms in chronic depression are by definition chronic and very difficult to change, even with extensive treatments, it is unlikely that change in symptoms during the baseline represented real symptomatic improvements. A more likely explanation would be that repeated assessments of symptoms with the same questionnaires introduces a measurement artifact, as has previously been shown in healthy individuals (Longwell \& Truax, 2005), an issue that my co-authors and I are currently investigating in a separate study.

In chapter 10 we investigated temporal relations between changes in depressive symptom severity during schema therapy for chronic depression and two potential mechanisms of change, the therapeutic alliance and core-beliefs. Results from these analyses are limited because we focused on only two potential mechanisms of change in schema therapy. Other specific and non-specific factors might also account for change in depressive symptoms during schema therapy for chronic depression and should be investigated in future studies. Moreover, due to the limited sample size and the lack of a control group, we were not able to test more complex models of how potential mechanisms might be related to changes in symptoms and we were not able to conduct formal mediation analyses to test, for example if the effects of schema therapy on depressive symptoms are mediated by change in core-beliefs. Moreover, we assessed explicit ratings of core-beliefs and this might not adequately tap the schema change processes underlying schema therapy. The appropriate timing of assessments and specific types of assessments (implicit vs explicit) of schemas therefore remains a challenge for future research.

\section{Clinical and research implications}

A number of relevant clinical and research implications can be drawn from the findings of the studies presented in this dissertation. When confronted with personal relevant emotional information, on a neural level, patients with chronic depression show blunted emotional responses (chapter 2). Clinically, this suggests that patients with chronic depression might benefit from treatments for depression that focus on increasing emotional processing such as emotion focused therapy (Greenberg \& Watson, 2006) or schema therapy (Young, Klosko, \& Weishaar, 2003). Future neuroimaging studies should test the effects of these treatments on emotionally blunted neural responses in patients with chronic depression. Findings from chapter $\mathbf{3}$ suggest that following a sad moodinduction, patients with chronic major depressive disorder show increased functional connectivity of resting state networks associated with schematic processing and rumination. Clinically, this suggests that patients with chronic depression might benefit from treatments that aim to decrease activation of negative schemas that might be triggered by negative mood. The degree to which connectivity within these networks 
following a sad mood induction remains stable following treatment remains to be investigated.

Our findings in part II of this dissertation point towards potentially important underlying vulnerability factors to depression and the stability of these factors following treatment. Patients with depression in chapter $\mathbf{4}$ were characterized by a distinct set of early maladaptive schemas. In chapter $\mathbf{9}$ we were able to shown that treatment for chronic depression focusing on these schemas (schema therapy) has positive effect on depressive symptoms. Future research should aim to further determine which early maladaptive schemas are specific for patients with chronic depression and whether addressing these schemas in treatment results in positive effects. The focus in schema therapy in recent years has shifted from a focus on early maladaptive schemas towards a focus on schema modes. Schema modes, defined as the moment to moment emotional states of patients have not been studied substantially in depression. As we have argued in chapter 8 of this dissertation, future research should identify which specific schema modes are predominantly activated in patients with (chronic) depression. Our results suggest that patients with depression might benefit from a stronger focus on early maladaptive schemas (chapter 4), interpersonal problems (chapter 6) or psychosocial functioning (chapter 7). Although current treatments for depression seem to have positive effects on each of these underlying vulnerability factors, findings of this dissertation indicate that these effects were weaker than the effects on depressive symptoms (e.g. chapter 4, chapter 6), suggesting that the underlying vulnerability to depression, in terms of these factors, remains at least partly intact following current treatments for depression. Addressing these vulnerability factors using an integrative treatment approaches such as schema therapy for chronic depression might be fruitful.

Our findings from chapter 7 suggest that more treatment sessions might be necessary to repair impaired psychosocial functioning in depression. These findings are in line with findings of a meta-analysis suggesting that patients with chronic depression might benefit from additional treatment sessions (Cuijpers et al., 2010). Clinically, such findings suggest that it might be necessary to provide long term psychotherapeutic treatments to patients with chronic depression. Future research should further aim to determine the optimal dose and frequency of psychotherapy for chronic depression. Our findings from chapter 9 suggest that schema therapy might be an effective treatment for patients with chronic depression. These findings add to the accumulating evidence that schema therapy is an effective treatment for patients with (chronic) depression (Carter et al., 2013; Malogiannis et al., 2014). Future research needs to determine the effects of schema therapy for chronic depression in larger samples and more controlled settings. Moreover, comparative efficacy studies in which schema therapy is compared to other treatment options for chronic depression are needed. 


\section{Implications for current definitions of treatment effects}

Studies in part II of this dissertation have investigated the effect of different treatments for depression on various presumably underlying vulnerability factors to depression. Currently treatment effects in treatment outcome studies of depression are usually defined in terms of relative decrease in depressive symptom severity (response) absolute decrease in depressive symptom severity (remission) or absence of diagnostic criteria (recovery). Thus current definitions on treatment effects exclusively rely on treatment effects on depressive symptoms. According to the world health organization, health is defined as "...a state of complete physical, mental and social well-being and not merely the absence of disease or infirmity" (World Health Organization, 1948). Thus, in order to come to a more complete definition of psychological health, it might be valuable to broaden current definitions, for example, by including improvements in psychosocial functioning rather than purely relying on symptomatic improvements when evaluating treatment effects.

Chronic depression, like other chronic mental disorders, is complex and multifaceted. Treatment effects might therefore not be adequately covered by only assessing symptomatic improvement. It might be valuable to reconsider symptomatic improvements as main treatment outcome in chronic depression and rather focus on the effects of treatments in terms of how patients learned to cope with depressed mood following treatment. For example, it might be more important from a patient and economical/societal perspective that chronically depressed patients are able to engage in recreational or work related activities despite depressed mood rather than focus on the mood itself. As an analogy, in the field of chronic pain research the relevant outcome is often how much patients engage in physical activities, despite their pain, rather than the pain intensity itself. A similar approach might prove useful in the field of chronic depression.

\section{Future directions}

The studies described in part I of this dissertation provide the first evidence of the underlying neuropathology related to self-referential processing and modulation of default mode network connectivity by sad mood in patient with chronic depression. In addition to the general implications of these studies for future research, our personal research agenda includes a follow-up study of the same participants described in part I, to assess the effects of schema therapy on the emotional blunted neural responses (chapter 2) and increased default mode network connectivity following the sad mood challenge (chapter 3). Due to time constrains this follow-up study could not be included in this dissertation. The single case series study described in chapter 9 of this dissertation is among the pioneering studies investigating the effects of schema therapy for chronic depression and chapter $\mathbf{1 0}$ is the first extensive analyses of potential mechanisms of change in schema therapy for chronic depression. In addition to the general research implications of these studies, our personal research agenda includes a 
follow-up evaluation to test the long term effects of schema therapy for chronic depression and the investigation of other pathways of change within that context, which could also not be included in the current dissertation due to time constrains. Finally, with regard to the unexpected decrease in symptoms during the baseline period of the single case series, my co-authors and I have started a study in patients with depression who are on a waitlist to start with treatment. In this study patients are randomly assigned to a condition in which they are asked to complete questionnaires of depressive symptom severity once a month or once a week in order to test whether assessment frequency introduces a measurement artifact. The results of this study will shed more light onto the "baseline effect" observed in chapter 9.

\section{Overall conclusion}

Depression is a common mental disorder that often takes a chronic course. Chronic depression has substantial negative consequences for the individual and the society. In addition to depressive symptoms, patients with depression are characterized by distinct maladaptive schemas, high levels of dysfunctional personality traits, interpersonal problems and impairments in psychosocial functioning. Although treatment for depression seems to have positive effects on these underlying vulnerability factors, the improvements are often not as strong as improvements in depressive symptom severity. This suggests that the underlying vulnerability to depression, in terms of these factors, remains at least in part intact after treatment, which might explain relapse, recurrences and chronicity to some extent. Treatment for depression might benefit from an additional focus on these underlying vulnerability factors, especially in patients who report stronger levels of these vulnerability factors, such as patients with chronic depression. In this regard, it might be particularly valuable to take an integrative treatment approach, such as schema therapy, to target these distinct vulnerability factors. In the current dissertation it was shown that schema therapy had positive effects on relevant outcomes in patients with chronic depression. Future studies should replicate these findings in larger samples, comparing schema therapy to other treatment options for chronic depression. 


\section{References}

Calvete, E., Estévez, A., López de Arroyabe, E., \& Ruiz, P. (2005). The Schema Questionnaire-Short Form: Structure and Relationship with Automatic Thoughts and Symptoms of Affective Disorders. European Journal of Psychological Assessment, 21(2), 90-99.

Carter, J. D., McIntosh, V. V., Jordan, J., Porter, R. J., Frampton, C. M., \& Joyce, P. R. (2013). Psychotherapy for depression: a randomized clinical trial comparing schema therapy and cognitive behavior therapy. Journal of Affective Disorders, 151(2), 500-505. doi: 10.1016/j.jad.2013.06.034

Cuijpers, P., van Straten, A., Schuurmans, J., van Oppen, P., Hollon, S. D., \& Andersson, G. (2010). Psychotherapy for chronic major depression and dysthymia: A meta-analysis. Clinical Psychology Review, 30(1), 51-62. doi: 10.1016/j.cpr.2009.09.003

Disner, S. G., Beevers, C. G., Haigh, E. A. P., \& Beck, A. T. (2011). Neural mechanisms of the cognitive model of depression. Nature Reviews. Neuroscience, advance online publication.

Greenberg, L. S., \& Watson, J. C. (2006). Emotion-Focused Therapy for Depression. Washington: American Psychological Association.

Harris, A. E., \& Curtin, L. (2002). Parental perceptions, early maladaptive schemas, and depressive symptoms in young adults. Cognitive Therapy and Research, 26(3), 405-416. doi: 10.1023/a:1016085112981

Horowitz, L. M., Rosenberg, S. E., Baer, B. A., Ureno, G., \& Villasenor, V. S. (1988). Inventory of interpersonal problems: Psychometric properties and clinical applications. Journal of Consulting and Clinical Psychology, 56(6), 885-892.

Horvath, A. O., Del Re, A. C., Fluckiger, C., \& Symonds, D. (2011). Alliance in individual psychotherapy. Psychotherapy (Chic), 48(1), 9-16. doi: 10.1037/a0022186

Jarrett, R. B., \& Thase, M. E. (2010). Comparative efficacy and durability of continuation phase cognitive therapy for preventing recurrent depression: Design of a double-blinded, fluoxetine- and pill placebo-controlled, randomized trial with 2-year follow-up. Contemporary Clinical Trials, 31(4), 355-377. doi: 10.1016/j.cct.2010.04.004-

Karsten, J., Penninx, B. W. J. H., Riese, H., Ormel, J., Nolen, W. A., \& Hartman, C. A. (2012). The state effect of depressive and anxiety disorders on big five personality traits. Journal of Psychiatric Research, 46(5), 644-650. doi: http://dx.doi.org/10.1016/j.jpsychires.2012.01.024

Keller, M. B., McCullough, J. P., Klein, D. N., Arnow, B., Dunner, D. L., Gelenberg, A. J., . . Zajecka, J. (2000). A comparison of nefazodone, the cognitive behavioral-analysis system of psychotherapy, and their combination for the treatment of chronic depression. The New England Journal of Medicine, 342(20), 1462-1470. doi: 10.1056/NEJM200005183422001

Longwell, B. T., \& Truax, P. (2005). The differential effects of weekly, monthly, and bimonthly administrations of the beck Depression Inventory-II: Psychometric properties and clinical implications. Behavior Therapy, 36(3), 265-275. doi: http://dx.doi.org/10.1016/S00057894(05)80075-9

Malogiannis, I. A., Arntz, A., Spiropoulou, A., Tsartsara, E., Aggeli, A., Karveli, S., . . Zervas, I. (2014). Schema Therapy for patients with chronic depression: A single case series study. Journal of Behavior Therapy and Experimental Psychiatry, 45(3), 319-329. doi: http://dx.doi.org/10.1016/j.jbtep.2014.02.003

Marchetti, I., Koster, E. H., Sonuga-Barke, E. J., \& De Raedt, R. (2012). The default mode network and recurrent depression: a neurobiological model of cognitive risk factors. Neuropsychology Review, 22(3), 229-251. doi: 10.1007/s11065-012-9199-9

Peeters, F., Berkhof, J., Rottenberg, J., \& Nicolson, N. A. (2010). Ambulatory emotional reactivity to negative daily life events predicts remission from major depressive disorder. Behaviour Research and Therapy, 48(8), 754-760. doi: 10.1016/j.brat.2010.04.008

Riso, L. P., Froman, S. E., Raouf, M., Gable, P., Maddux, R. E., Turini-Santorelli, N., . . Cherry, M. (2006). The Long-Term Stability of Early Maladaptive Schemas. Cognitive Therapy and Research, 30(4), 515-529. 
Spijker, J., van Straten, A., Bockting, C. L. H., Meeuwissen, J. A. C., \& van Balkom, A. J. L. M. (2013). Psychotherapy, antidepressants, and their combination for chronic major depressive disorder: A systematic review. The Canadian Journal of Psychiatry / La Revue canadienne de psychiatrie, 58(7), 386-392.

Vittengl, J. R., Clark, L. A., \& Jarrett, R. B. (2003). Interpersonal problems, personality pathology, and social adjustment after cognitive therapy for depression. Psychological Assessment, 15(1), 29-40.

Wang, C. E. A., Halvorsen, M., Eisemann, M., \& Waterloo, K. (2010). Stability of dysfunctional attitudes and early maladaptive schemas: A 9-year follow-up study of clinically depressed subjects. Journal of Behavior Therapy and Experimental Psychiatry, 41(4), 389-396.

Wiersma, J. E., van Schaik, D. J. F., Hoogendorn, A. W., Dekker, J. J., Van, H. L., Schoevers, R. A., Blom, M. B. J., Maas, K., Smit, J. H., McCullough Jr, J. P., Beekman, A. T. F., van Oppen, P. (in press). The effectiveness of the cognitive behavioral analysis system of psychotherapy for chronic depression: A randomized controlled trial. Psychotherapy and Psychosomatics. doi:10.1159/000360795

World Health Organization. (1948). Preamble to the Constitution of the World Health Organization as adopted by the International Health Conference, New York, 19-22 June, 1946.

Young, J. E., Klosko, J., \& Weishaar, M. E. (2003). Schema therapy: A practitioner's guide. New York: Guilford Press. 

Summary 
SUMMARY 
Major depressive disorder is a common and highly disabling mental disorder that often takes a chronic course. A depressive disorder is assumed to be chronic when the symptoms of depression persist for two years or longer. Previous research has shown that compared to non-chronic depression, chronic depression is associated with more negative health and economic outcomes, highlighting the importance of effective treatments for chronic depression. Current treatments for chronic depression are available but are often less effective than treatment for episodic forms of depression. Possible reasons for the lack of effectiveness of current treatment approaches might be that current treatment options are too time-limited and too symptom focused. The central question of the current dissertation was if better treatment outcomes could be achieved in long-term psychotherapy for chronic depression that focusses on the underlying vulnerability to chronic depression.

The primary aim of the current dissertation was to test the effects and explore the underlying mechanisms of a long-term integrative treatment approach (schema therapy) for patients with chronic depression. The secondary aim was to further examine underlying neurological and psychological vulnerability factors to (chronic) depression and to determine the stability of psychological vulnerability factors during treatment for depression.

Chapter 1 of this dissertation provides an overview of the diagnostic criteria of a major depressive disorder, the conceptualization of different forms of chronic depression, the prevalence and consequences of chronic depression and a definition and overview of treatment effects of current common treatment approaches to chronic depression. Developmental, psychological and neurological vulnerability factors to (chronic) depression are described. The chapter ends with an outline of the goals of the current dissertation.

In chapter 2 , the neural correlates of self-referential processing in patients with chronic major depressive disorder were examined. We used functional magnetic resonance imaging (fMRI) during a passive self-referential processing paradigm in patients with chronic major depressive disorder $(n=17)$ and matched healthy controls $(n=18)$. We found that patients with chronic depression had decreased BOLD signal during processing of negative depression related personality traits vs. neutral words in the medial prefrontal cortex. Compared to patients, controls had increased BOLD signal in the dorsolateral prefrontal cortex during processing of positive vs. neutral personality trait words. These findings suggest that chronic depression is characterized by distinct neural processing of emotional self-relevant stimuli in these brain areas. We interpreted these findings in terms of a blunted emotional response in patients with chronic depression.

In chapter 3, the default mode network functional connectivity during rest in patients with chronic depression and matched controls before and after a sad mood-induction 
was investigated. The mood induction procedure resulted in decreased mood ratings in patients with chronic depression and in non-patient controls. Posterior cingulate cortex (PCC) connectivity with the left parahippocampal gyrus, the left superior temporal gyrus and the left anterior inferior temporal cortex increased in patients with chronic depression, relative to controls, following the sad mood induction. In contrast, PCC connectivity with the left anterior prefrontal cortex and the right precuneus decreased in patients with chronic depression and increased in controls following the sad mood induction. We interpreted these findings in terms of activation of negative latent schemas or rumination of negative thoughts in patients with chronic depression, triggered by the mood induction. Connectivity in non-patient controls following the mood induction was interpreted as indicative of a cognitive control (coping) mechanism possibly preventing non-patient controls from retrieving negative memories and engaging in ruminative thinking.

Next to neurological disturbances underlying chronic depression, we also tested the stability of psychological vulnerability factors during treatment for depression. The study described in chapter 4 tested the stability of early maladaptive schemas over a course of outpatient treatment for depression as well as the relation of schemas with depressive symptoms in 132 patients with a major depressive disorder. Specific schemas (failure, emotional deprivation, abandonment/instability) were found to be related to depressive symptom severity. These schemas, as well as other schemas that were unrelated to depressive symptoms, remained relatively stable over the course of treatment. Our interpretation of these findings was that the underlying vulnerability to depression, in terms of early maladaptive schemas, remains intact following outpatient treatment for depression, highlighting the potential value of addressing these schemas in schema therapy.

Chapter $\mathbf{5}$ is based on data of a longitudinal cohort study, the Netherlands Study for Depression and Anxiety. Based on the data of this study, we investigated the two-year stability of the personality traits neuroticism and extraversion in treated and untreated patients with depression. We found that neuroticism and depressive symptoms decreased in treated and untreated people with depression whereas extraversion did not change significantly. We discuss these findings in terms of the mood-state dependency of neuroticism and concluded that assessment of personality traits in depression is clouded by the current mood state.

The relation between interpersonal problems, the therapeutic alliance and depressive symptoms during cognitive therapy for depression was examined in chapter 6 . Following a 16 to 20 session course of cognitive therapy, interpersonal distress improved whereas the interpersonal style of depressed patients remained largely stable. Patients who scored high on agency/dominance prior to treatment reported lower quality therapeutic alliance whereas patients who scored high on communion/affection prior to treatment reported higher quality alliance. Patients experiencing higher 
interpersonal distress prior to treatment reported higher symptom severity throughout treatment. These findings suggest that interpersonal problems of patients with depression have some predictive validity with respect to the therapeutic alliance and treatment outcomes.

Chapter 7 describes the findings of a meta-analysis quantifying the effects of psychotherapy for depression on improvements in psychosocial functioning. We identified 31 randomized controlled trials comparing the effects of psychotherapy to a control group that also included a measure of psychosocial functioning at post treatment. Psychotherapy resulted in significant improvements in psychosocial functioning at posttreatment with moderate effect sizes. In multivariate meta-regression analyses, the effect size of depressive symptoms, the number of treatment sessions and the treatment format (individual versus group) remained as significant predictors of the effect size for psychosocial functioning. We concluded that psychotherapy has positive effects on psychosocial functioning in depression and that these effects are highly related to, but partly independent from the effects of psychotherapy on depressive symptom severity.

In chapter 8 the theoretical application of schema therapy for chronic depression is described. Based on our own research and similar studies we argue that chronic depression is characterized by a number of psychological and developmental vulnerability factors (adverse childhood events, personality pathology, cognitive factors, interpersonal factors) and that current treatments for depression might not adequately address these factors. We provide examples of how these factors might be addressed in schema therapy for chronic depression and provide a treatment manual of schema therapy for chronic depression.

The results of a single case-series study of schema therapy for chronic depression are described in chapter 9. In this study 25 patients with a chronic major depressive disorder were first assessed during a 6 to 24 weeks baseline period. Following this, patients entered a 12 weeks exploration phase of the study during which schemas were explored, the case-conceptualization was done and treatment goals were set. After the exploration phase, patients received up to 65 sessions individual schema therapy. Of the 25 patients who started with the study, five dropped out during the baseline phase. Of the remaining 20 patients who started with treatment, no one dropped out, $40 \%$ responded and 35\% remitted. Of the 12 patients who were followed until treatment completion, 58\% recovered at the end of the intervention. Mixed model regression analyses contrasting exploration phase and intervention phase effects with the baseline phase yielded significant effects of the intervention on depressive symptom severity, global symptomatic distress and overall wellbeing. These findings provide preliminary evidence for the effectiveness of schema therapy for chronic depression.

In chapter 10 the underlying mechanisms of change in schema therapy for chronic depression were explored. In particular, we investigated temporal relations between 
change in depressive symptoms during schema therapy for chronic depression and change in negative core-beliefs as well as the relation between change in symptoms and the therapeutic alliance. Our findings have shown that there are no temporal relations between changes in negative core-beliefs and change in depressive symptoms during schema therapy for chronic depression. Instead, changes in negative core-beliefs are concurrently associated with changes in depressive symptoms. Moreover, change in depressive symptoms was unrelated to ratings of the therapeutic alliance but alliance ratings were at least to some degree colored by the current mood state.

In chapter 11, the findings of this dissertation are discussed. A number of methodological weaknesses as well as clinical and research implications are discussed. It is argued that depression is characterized by a number of underlying vulnerability factors and that patients with depression might benefit from a focus on these factors. Our findings indicate that schema therapy might be a valuable treatment option for patients with chronic depression and shed some light on the underlying mechanisms of change. The overall conclusion that can be drawn at this point is that schema therapy is a promising treatment for chronic depression but that randomized clinical trials are needed to demonstrate the effectiveness. 


\section{Valorization addendum}


VALORIZATION 
This dissertation describes the results of a number of studies on the underlying vulnerability to (chronic) depression and on the psychological treatment of chronic depression with schema therapy. In describing the knowledge valorization of the results of the current dissertation, the focus is on the societal and economic relevance of the findings, potential target groups, possible activities and/or products and on the innovative aspects of the studies conducted for this dissertation. Examples of how knowledge valorization has been achieved and ideas for how knowledge valorization can further be realized are described at the end of the addendum.

\section{Societal and Economic Relevance}

Depression is a common mental disorder that is associated with negative consequences from a clinical, societal and economic point of view (World Health Organization, 2004). The economic burden of depression is due to the direct costs of the disorder (i.e. costs of treatment) as well as indirect costs (e.g. work absence). According to the Dutch National Institute for Public Health and the Environment (Rijksinstitut voor Volksgezondheid en Milieu; RIVM), depression is one of the most expensive illnesses with annual costs of approximately 1.6 billion euro in the Netherlands (RIVM, 2013). The UK office for national statistics (ONS) has estimated that 13.3 million work days were lost in 2011 due to depression (ONS, 2012). Especially chronic forms of depression seem to be associated with these negative consequences. The costs of depression can be explained by the duration of symptoms, thus by the chronicity of the disorder (Fostick, Silberman, Beckman, Spivak, \& Amital, 2010). This situation calls for effective treatments for patients with (chronic) depression. Although effective treatments for depression exist, about half of the patients with depression do not respond to treatment (Cuijpers et al., 2014), an unacceptably low rate of response.

Given these numbers, research into the underlying vulnerability factors of (chronic) depression and research exploring new treatment options for patients with chronic depression is of great relevance on a clinical, societal as well as economic level. Although the findings of the studies described in this dissertation do not directly contribute to decreased health care costs, they point towards new treatment targets that might ultimately decrease treatment costs. The cost-effectiveness of schema therapy has been demonstrated previously, in patients with personality disorders (e.g. Bamelis et al, in prep; van Asselt et al., 2008). Although in the current dissertation, my co-authors and I did not formally assess the cost-effectiveness of schema therapy, the studies described in the current dissertation set the stage for future studies that might inform on the costeffectiveness of schema therapy for chronic depression which, when implemented might have direct relevance on economic costs.

\section{Target groups}

The findings of this dissertation are relevant for psychotherapy researchers, for professionals working in clinical settings, patients suffering from chronic depression 
and stakeholders from the industry and technology sector. The results of the studies of this dissertation show that current treatments for depression do not adequately touch the underlying vulnerability to depression and that a new psychological treatment approach, schema therapy, has positive effects on depressive symptoms in patients with chronic depression. Although it is too premature to implement schema therapy for chronic depression in the mental health care system, professionals, working in mental health care might use specific therapeutic techniques that are described in this dissertation. The studies described in this dissertation provide the underlying rationale of using these techniques in the psychological treatment of patients with chronic depression. The industry and technology sector has started to translate psychosocial interventions for mental disorders into (online) computerized mental health interventions and/or smartphone applications. As the evidence of schema therapy for patients with chronic mental health problems is increasing, the interest of third parties in such applications might grow and similar applications might be developed based on the principles and techniques used in schema therapy. Thereby, the findings from the studies described in this dissertation are indirectly contributing to the knowledge utilization outside the academic sector. Specifically, the results of the study described in chapter 9 of this dissertation contribute to the emerging evidence of schema therapy for chronic depression and thereby promotes interest in schema therapy by stakeholders within and outside the academic community.

\section{Activities and Products}

The findings of the studies described in the current dissertation point towards possibilities for activities and/or products. While it is too premature to implement schema therapy for chronic depression as a standard treatment in the mental health care sector, workshops and training programs can be organized by schema therapists to teach health care professionals specific schema therapy techniques. Findings from the studies of the current dissertation provide the rationale for using these techniques in the treatment of patients with chronic depression. For example, health care professionals might organize workshops in the context of scientific conferences during which schema therapy techniques that can be used in the treatment of patients with chronic depression are taught to other health care professionals. Moreover, specific therapeutic techniques used in schema therapy might be integrated into formal psychotherapy training modules. Schema therapists might also produce formal training videos or other educational material to promote training in schema therapy. The findings from chapter 8 and chapter 9 provide a rationale and preliminary evidence of applying schema therapy to chronic depression and thus for these activities/products.

\section{Innovation}

The studies conducted in this dissertation are innovative as they adress novel research questions, many of which have not previously been explored. The study described in 
chapter 3 of this dissertation, for example, adapted procedures used in experimental psychology (sad mood induction) during neuroimaging of patients with a current chronic depression. This allowed for studying neural reactions to a sad mood challenge in currently depressed individuals, which has not been studied before.

One major innovation of the single case series study described in chapter 9 of this dissertation is the large number of repeated assessments throughout the study period. This allowed for a more dynamic modeling of change in depressive symptoms throughout the different study phases. Based on this innovative study design we could show, for example, that durign a period in which no treatment was provided, depressive symptoms improved initally, before returning to a baseline again.

The findings described in chapter 10 of this dissertation are based on a novel, innovative approach of studying underlying mechanisms of change in psychotherapy research. Whereas traditional treatment studies have focussed on between-person differences, the study described in chapter 10 has focussed on changes withinindividuals over time. This allowed for studying potential mechanisms of change in schema therapy for chronic depresison in a realtively small sample and to disentagle the temporal relations between study variables, an often neglected prerequisite in processoutcome research.

\section{Schedule and Implementation}

Some of the studies described in this dissertation have already evoked interest outside of the scientific community which has resulted in traditional and social media apperances. Due to these media apperances, some of the resutls of the studies of this dissertation have been communicated to a broader audience, fostering public engagement. For example, the study described in chapter 7 of this dissertation has been featured on "The Mental Elf" website $(04 / 2014)$, a mental health blog reaching more thant 30.000 followers/subscribers at various professional levels. Public engagement in study results was also promoted by presenting the rationale of chapter 9 during a newspaper and during a TV live interview. In addition to this, studies from this dissertation have been translated into Dutch and German and published in clinical journals that are more likely to reach health care professionals who are not working in a research setting. Moreover, therapists who were involved in studies described in this dissertation have organized workshops of schema therapy for chronic depression for health care professionals thereby uitilizing knowlwedge derived from studies of this dissertation. Knowledge valorization will further be stimulated by communicating findings of this dissertation not only within the scientific community (journal publications, conference presentations) but also outside the scientific community (for example via press releases). 


\section{References}

Bamelis, LLM, Arntz, A, Wetzelaer, P, Verdoorn, R, Evers, SMAA. Economic evaluation for personality disorders: a multicenterd randomized trial. Submitted manuscript.

Cuijpers P, Karyotaki E, Weitz E, Andersson G, Hollon SD, van Straten A. (2014) The effects of psychotherapies for major depression in adults on remission, recovery and improvement: A metaanalysis. J Affect Disord, 159, 118-126.

Fostick, L., Silberman, A., Beckman, M., Spivak, B., \& Amital, D. (2010). The economic impact of depression: Resistance or severity? European Neuropsychopharmacology, 20(10), 671-675.

Office of National Statistics (2012). Sickness Absence in the Labour Market, April 2012.

RIVM. Kosten van Ziekten in Nederland. Bilthoven: RIVM, http://www.kostenvanziekten.nl;, versie 1.3, 26 november 2013.

van Asselt, A. D., Dirksen, C. D., Arntz, A., Giesen-Bloo, J. H., van Dyck, R., Spinhoven, P., . . Severens, J. L. (2008). Out-patient psychotherapy for borderline personality disorder: cost-effectiveness of schema-focused therapy v. transference-focused psychotherapy. British Journal of Psychiatry, 192(6), 450-457.

World Health Organization. The global burden of disease: 2004 update. 2008, Geneva, Switzerland: World Health Organization. 
Acknowledgements 
ACKNOWLEDGEMENTS 
I would like to thank everybody who has supported me during the last five years and who has contributed to this dissertation. You all made my PhD years a great experience.

First of all I would like to thank my promotors, Prof. Marcus Huibers and Prof. Arnoud Arntz and my co-promotor, Prof. Frenk Peeters.

Marcus, you have been a fantastic supervisor who always supported me. We met during the research master program and when you told me that you have a job opening for a $\mathrm{PhD}$ position I immediately knew that this position would be the perfect match. I want to thank you for your trust and confidence in me that I would make this PhD project a success and for pushing me during these years. In the first week of my work as a PhD student you told me that "the goal is to become world famous" and that "the sky is the limit". I'm afraid that I did not succeed in this but you always took good care of me and provided me with all the opportunities to develop and grow as a researcher and I'm very thankful for that. You have taught me that the key fundament for a successful research project is a good method and I hope that this dissertation can be regarded as proof that we succeeded in implementing this. You also have been very generous in offering an extension, when things were going slower than we had hoped for and in supporting extended work visits abroad (Leuven, Philadelphia). I have learned a lot from you, not only research-wise but also life-wise. We also had great fun at conferences (Boston, New York, Reykjavík, Marrakesh, Den Haag) and I learned another important lesson from you during these trips: "work hard play hard". Marcus, thank you for everything and I really hope we will continue to work together.

Arnoud, you have been a great supervisor and a great mentor for me. You have supported me already during the research master when you involved me in a study that resulted in my first co-authored publication and I am very grateful for this. You always had an open door when I had more or less urgent questions about multi-level analyses, the correct covariance structures and so on. The time and efforts you took for this was not only very helpful for working towards getting the studies published but also a great learning experience for me. I could always count on your support and it was a great privilege to work with you and I hope that this will be continued.

Frenk, thank you for all your support before and during my PhD project. During the research master program you helped me to arrange my research internship at the Psychiatry Department at the University of Pennsylvania. This research experience made me realize that I wanted to pursue a career in research. You also always supported me at all stages of the PhD project, for example when we struggled with a slow intake of new participants. Many thanks for everything.

Although not officially co-promotor, I would also like to thank Jill Lobbestael for being a great mentor. Jill, thank you for all your input in the schema therapy project from the 
beginning to the end and for giving me the opportunity to extent my contract and working together with you on one of your Veni studies.

I would like to thank all patients who participated in the schema therapy study for all their efforts and for bringing up the motivation and energy of completing all the questionnaires every week. Obviously there would be no dissertation without your commitment. Thank you.

I want to thank the therapists at the Riagg Maastricht who have provided schema therapy in the case series study: Ina Leeuw, Christel Achterberg, Claudia Mordang and Annemiek Dobbelaar. Thank you for your commitment to the study and for taking on the extra workload associated with the study. Ina, I want to thank you in particular for your special commitment in supporting the study from the beginning to the end and for all the efforts that you took to help making the schema therapy for chronic depression project a success.

Thanks are also due to all intakers of team 1 at the Riagg Maastricht for screening patients for the study and for taking on the extra work load that was associated with this: Claudia Mordang, Josien Schneiders, Danielle Ummels, Elisa Wensink, Marionne Wolfis, Andrea Smits, Iris Tan, Eline Mollema.

I would also like to thank the former team-manager of the mood disorder treatment section at the Riagg, Anja Kaarst, for her support and commitment of conducting this study at the Riagg Maastricht. Anja, thank you very much for supporting me when setting up the study and for your help with keeping the study running.

Thanks are also due to Hannie van Genderen for giving me the opportunity to work as a statistical analyst for the Riagg Maastricht.

I want to thank the management of the Riagg Maastricht for the financial support of my position and the clinical study.

I would like to thank everybody who has supported me in setting up and conducting the fMRI studies of my dissertation. Nicolette, thank you so much for all the efforts and for your patience when teaching me how to use the MRI scanner and in explaining and helping with the data-analysis. Obviously without your help I would not have succeeded in getting the two fMRI papers in my dissertation. Conny, thank you so much for spending all the hours with me on the resting state analyses. Linda, thank you for your many backup hours when scanning the last participants on the good old Allegra.

Thanks are due to all collaborators and co-authors from the studies for my dissertation: Lotte Bamelis, thank you for letting me use your data set for one of our studies. Michael Thase, thank you for hosting me in Philadelphia and for giving me the opportunity to 
publish my master thesis research together with you. Robin Jarrett, Lee Anna Clark and Jeffrey Vittengl, the conference calls and informal discussions were a great learning experience for me. Robert DeRubeis, dear Rob thank you for your hospitality when hosting me in Philadelphia. I very much appreciated our discussions and exchange of ideas on the mechanisms paper and the welcoming atmosphere at the DeRubeis lab in Philadelphia.

Thanks are due to the research coordinators, research assistants and support staff at Maastricht University who supported the studies described in my dissertation: Annie Hendriks and Annie Raven you were always very helpful with all the practical and organizational aspects of the studies and this contributed to the fact that the studies were running smoothly: As I learned, a phenomenon known as the "Annie effect". Annie $\mathrm{H}$, thank you so much for all your support and help in recruiting participants for my studies and for helping with keeping participants in the study. Annie R, finding matched control participants for the fMRI study was a big challenge. Thank you for your help with this. Rosanne, I would like to thank you for providing the infrastructure for online data collection and helping with the logistics of the studies. Over the years I have become an EMIUM expert and I would like to thank Rosanne and Sita for their time and patience in explaining everything and helping out quickly when necessary. I would also like to thank Michelle Houben and Elly Niesten for helping out with the SCID interviews. Thanks are also due to Michiel Vestjens for programming an online flash version of the implicit measures used in my studies and for Anne Roefs who developed this implicit measure and generously shared it for use in our studies.

I also want to thank all local research coordinators at the Riagg Maastricht for their support in patient recruitment and their help with the logistics of the study: Brigit Lok, Nicole van Loo, Christel Harisma and Marga Schnitzler.

I would like to thank Angela Verweij and Lindy Dullens for all their organizational help throughout the years.

Thanks are due to all students who have supported recruitment of participants and collected data, Patrick Schwarz, Danielle Jongen, Michiel van den Goor, Nora Krott, Lana Pulmanns.

I would like to thank the members of my evaluation committee: Prof. Peter Muris, Prof. David Bernstein, Prof. Claudi Bockting, Prof. Jan Spijker and Dr Jeffrey Roelofs for taking the time to read and to evaluate my dissertation manuscript.

Peter, I would also like to thank you for supporting the depression symposium that Lotte and I have organized. 
Lotte and Thomas, dear paranimfen: thank you for your support in the preparation of my defense. Dear Lotte you were my research sister and roomie from the beginning of the $\mathrm{PhD}$ and we went through all the phases of the PhD together: From the happy moments (last participant included party) to the "stupid-ass" moments (rejected papers). Thank you for the good time and keep up the "Lemmens \& Renner depression lab" here in Maastricht. Thomas, we know each other since we started studying in 2004. I always enjoyed chatting with you when you walked by the office with your coffee mug and I have good memories of EPP symposia, biertjes and billiard matches.

I also would like to thank all other PhD students at CPS for making me feel at home and all the fun we had at conferences, dinners, EPP symposia, retreats and previous defense parties.

Last but not least I would like to thank my family and friends from outside the UM for always being there for me and for supporting me when needed during these busy last five years. Elisabeth, vielen Dank für deine großzügige Unterstützung mein Leben lang. Du hast mich nie mit irgendwelchen Erwartungen unter Druck gesetzt und hast mich auf meinen Weg immer bedingungslos unterstützt. Dafür bin ich dir sehr dankbar. Klaus, auch dir danke ich für deine Unterstützung und Verständnis dafür, dass in den letzten Jahren oft die Zeit fehlte, um einen intensiveren Kontakt zu haben. Auch dir bin ich sehr dankbar für deine bedingungslose Unterstützung während meines Studiums und in der Zeit danach. Liebe Oma Rosa, auch dir möchte ich recht herzlich danken für deine Unterstützung während meines Studiums. Außerdem möchte ich mich beim Rest der Familie bedanken für euer Interesse an meiner Arbeit und euer Verständnis, wenn die Arbeit Vorrang hatte. Mein Dank gilt auch Jessicas Familie, insbesondere Annette und Dietrich, für die tolle Unterstützung in den letzten Jahren. Wir konnten uns immer auf euch verlassen in stressigen Zeiten und dafür bin ich euch sehr dankbar.

Dank geht auch an meine Freunde in Köln, dafür, dass ihr zu mir gehalten habt, auch wenn ich in den letzten Monaten abgetaucht bin und alles abgesagt habe, weil die Arbeit vorging.

Jessi, du warst die wichtigste Person für mich in all den Jahren. Du hast mir den Raum gegeben um meine Arbeit abzurunden und mich bedingungslos unterstützt. Du hast mich in den stressigen Wochen aufgemuntert und mir gezeigt, dass die Arbeit nicht alles ist und dafür liebe ich Dich. Die letzten Jahre waren eine spannende und aufregende Zeit. Auf ins nächste Abenteuer! 


\section{Curriculum Vitae}


CURRICULUM VITAE 
Fritz Renner was born in Cologne (Germany) on July 30th 1982 . He graduated from high school and obtained the German Abitur degree in Cologne in 2002. After this he conducted alternative Civilian Service ("Zivildienst") for 10 months at an urban geriatric psychiatric nursing home in Cologne. In 2004 he started to study Psychology, with a specialization in Cognitive Psychology, at Maastricht University (The Netherlands). During the bachelor program he spent six months at Lund University (Sweden). After obtaining his Bachelor of Science degree in 2004, he joined the Research Master program in Cognitive Neuroscience, Neuropsychology and Psychopathology with a specialization in Psychopathology at Maastricht University. In the research master he received the prestigious Huygens fellowship by the Dutch Ministry of Education, Culture and Science. During the research master program he conducted a three months clinical internship at the Cologne Early Recognition and Intervention Centre for Mental Crises, University Hospital Cologne (Germany) and a six months research internship at the Mood and Anxiety Disorders Research Unit, Department of Psychiatry, University of Pennsylvania (USA). During the research master he worked as a student assistant at the Department of Psychiatry at Maastricht University. In September 2009 he graduated cum-laude from the research master program and started his $\mathrm{PhD}$ project at the Department of Clinical Psychological Science at Maastricht University. During the PhD project he worked at the community mental health center (RIAGG Maastricht) as a clinical psychologist one day per week for five months. During the PhD he received two micro grants for the development and evaluation of an online peer-review system for students. As part of his $\mathrm{PhD}$ project he visited the Methodology and Statistics of Educational Sciences Research Group at the University of Leuven (Belgium) for one month and the Department of Psychology, University of Pennsylvania (USA) for one month. 
CURRICULUM VITAE 


\section{Publications}

Journal Articles

Renner, F., Bamelis, L. L. M., Huibers, M. J. H., Speckens, A., \& Arntz, A. (in press). The impact of comorbid depression on treatment outcomes in personality disorders: Results of a randomized controlled trial. Behavioral Research and Therapy.

Dinger, U., Barrett, M. S., Zimmermann, J., Zilcha-Mano, S., Schauenburg, H., Wright, A. G. C., Renner, F., \& Barber, J. P. (in press). Depressive Experiences: Dependency, SelfCriticism and Interpersonal Problems in Major Depression. Journal of Clinical Psychology.

Renner, F., Cuijpers, P., \& Huibers, M. J. H. (2014). The effect of psychotherapy for depression on improvements in social functioning: a meta-analysis. Psychological Medicine, 44 (14), 2913-2926.

Werthmann, J., Renner, F., Roefs, A., Huibers, M. J. H., Plumanns, L., Krott, N., \& Jansen, A. (2014). Looking at food in sad mood: Do attention biases lead emotional eaters into overeating after a negative mood induction? Eating Behaviors, 15 (2), 230-236.

Renner, F., Arntz, A., Leeuw, I., \& Huibers, M. J. H. (2014). Schematherapie für chronische Depressionen [Treatment for chronic depression using schema therapy]. Verhaltenstherapie, 24, 169-181.

Renner, F., Arntz, A., Leeuw, I. \& Huibers, M. J. H. (2014). Behandeling van chronische depressie met schema therapie [Treatment for chronic depression using schema therapy]. Tijdschrift voor Psychotherapie, 40 (4), 256-272.

Renner, F., Schwarz, P., Peters, M. L., \& Huibers, M. J. H. (2014). Effects of a best-possibleself mental imagery exercise on mood and dysfunctional attitudes. Psychiatry Research, 215 (1), 105-110.

Moradveisi, L., Huibers, M. J. H., Renner, F. \& Arntz, A. (2014). The influence of patients' preference/attitude towards psychotherapy and antidepressant medication on the treatment of major depressive disorder. Journal of Behavior Therapy and Experimental Psychiatry, 45, 170-177.

Renner, F., Van Goor, M., Huibers, M. J. H., Arntz, A., Butz, B., \& Bernstein, D. P. (2014). Effecten van schemagroepstherapie bij jongvolwassenen [Effects of group schematherapy for adolescents]. Gedragstherapie, 47 (1), 20-36. 
Renner, F., Van Goor, M., Huibers, M. J. H., Arntz, A., Butz, B., \& Bernstein, D. P. (2013). Short-term group schema cognitive-behavioral therapy for young adults with personality disorders and personality disorder features: Associations with changes in symptomatic distress, schemas, schema modes and coping styles. Behavioral Research and Therapy, 51, 487-492.

Schultze-Lutter, F., Renner, F., Paruch, J., Julkowski, D., Klosterkotter, J., \& Ruhrmann, S. (2013). Self-Reported Psychotic-Like Experiences Are a Poor Estimate of Clinician-Rated Attenuated and Frank Delusions and Hallucinations. Psychopathology, 47 (3), 194-201.

Renner, F., Arntz, A., Leeuw, I. \& Huibers, M. J. H. (2013). Treatment for chronic depression using schema therapy. Clinical Psychology Science and Practice, 20, 166-180.

Moradveisi, L., Huibers, M., Renner, F., Arasteh, M., \& Arntz, A. (2013). The influence of comorbid personality disorder on the effects of behavioural activation vs. antidepressant medication for major depressive disorder: Results from a randomized trial in Iran. Behaviour Research and Therapy, 51, 499-506.

Renner, F., Penninx, B. W. J. H., Peeters, F., Cuijpers, P., \& Huibers, M. J. H. (2013). Twoyear stability and change of neuroticism and extraversion in treated and untreated persons with depression: Findings from the Netherlands Study of Depression and Anxiety (NESDA). Journal of Affective Disorders, 150 (2), 201-208.

Moradveisi, L., Huibers, M. J. H., Renner, F., Arasteh, M., \& Arntz, A. (2013). 'Results for behavioural activation are overstated': Reply. The British Journal of Psychiatry, 202 (6), 466-467.

Moradveisi, L., Huibers, M. J. H., Renner, F., Arasteh, M., \& Arntz, A. (2013). Behavioural activation v. antidepressant medication for treating depression in Iran: randomised trial. The British Journal of Psychiatry, 202, 204-211.

Renner, F., Jarrett, R. B., Vittengl, J. R., Barrett, M. S., Clark, L. A., \& Thase, M. E. (2012). Interpersonal problems as predictors of therapeutic alliance and symptom improvement in cognitive therapy for depression. Journal of Affective Disorders, 138 (3), 458-467.

Renner, F., Lobbestael, J., Peeters, F., Arntz, A., \& Huibers, M. J. H. (2012). Early maladaptive schemas in depressed patients: Stability and relation with depressive symptoms over the course of treatment. Journal of Affective Disorders, 136 (3), 581-590.

Bamelis, L. L. M., Renner, F., Heidkamp, D., \& Arntz, A. (2011). Extended schema mode conceptualizations for specific personality disorders: an empirical study. Journal of Personality Disorders, 25 (1), 41-58. 


\section{Submitted manuscripts}

Renner, F., Siep, N., Lobbestael, J., Arntz, A., Peeters, F., \& Huibers, M. J. H. (2014). Neural correlates of self-referential processing and implicit self-associations in chronic depression. Submitted for publication.

Renner, F., Arntz, A., Peeters, F., Lobbestael, J., \& Huibers, M. J. H. (2014). Schema therapy for chronic depression: Results of a multiple single case series study. Submitted for publication.

Renner, F., Siep, N., Arntz, A., van de Ven, V., Peeters, F., Quaedflieg, C., \& Huibers, M. J. H. (2014). Sad mood induction modulates default mode network resting-state functional connectivity in chronic depression. Submitted for publication.

Lemmens, L. H. J. M., Renner, F., \& Peeters, F. (2014). Persisterende Depressieve Stoornis [Persistent Depressive Disorder]. In I. Franken, P. Muris \& D. Denys (Eds.), Basisboek Psychopathologie. Utrecht: de Tijdstroom. Book chapter submitted for publication.

\section{Conference Presentations}

Schema therapy for chronic depression: Testing potential mechanisms of change. Symposium presentation at the Congress of the European Association of Behavioral and Cognitive Therapies (EABCT) 2014. The Hague, the Netherlands.

Schema therapy for (chronic) depression. Symposium presentation at the world congress of the International Society of Schema Therapy (ISST) 2014. Istanbul, Turkey.

Schema therapy for chronic depression. Symposium presentation the Congress of the European Association of Behavioral and Cognitive Therapies (EABCT) 2013. Marrakesh, Morocco.

Self-referential processing in depressed patients and matched non-clinical controls: An fMRI study. Open paper presentation at the "Tagung experimentell arbeitender Psychologen" (TeaP) [Conference of Experimental Psychologists] 2013. Vienna, Austria.

Schemagerichte therapie voor chronische depressie [Schema therapy for chronic depression]. Invited talk at the "Psychiaterconferentie GGZ Noord-Holland-Noord" 2013. Heiloo, the Netherlands.

Single case series of schema-focused therapy for chronic depression: Preliminary findings. Symposium presentation at the world congress of the International Society of Schema Therapy (ISST) 2012. New York City, USA. 
Schemagerichte therapie voor chronische depressie [Schema therapy for chronic depression]. Symposium presentation at the Dutch Association for Cognitive Behavioral Treatments (VGCT) 2012. Veldhoven, the Netherlands.

Early maladaptive schemas in depressed patients: Structure, stability and relation with depressive symptoms. Open paper presentation at the Congress of the European Association of Behavioral and Cognitive Therapies (EABCT) 2011. Reykjavik, Iceland.

Interpersonal problems as predictors of therapeutic alliance and symptom improvement in cognitive therapy for depression. Open paper presentation at the Congress of the European Association of Behavioral and Cognitive Therapies (EABCT) 2011. Reykjavik, Iceland.

Early maladaptive schemas in depressed patients: Structure, stability and relation with depressive symptoms. Poster presented at the World Congress of Cognitive Behavioral Therapies (WCBCT) 2010. Boston, USA.

Interpersonal problems as predictors of therapeutic alliance and symptom improvement in cognitive therapy for depression. Poster presented at the World Congress of Cognitive Behavioral Therapies (WCBCT) 2010. Boston, USA.

Early maladaptive schemas in depressed patients: Structure, stability and relation with depressive symptoms. Open paper presentation at the International Society of Schema Therapy (ISST) Conference, 2010. Berlin, Germany. 


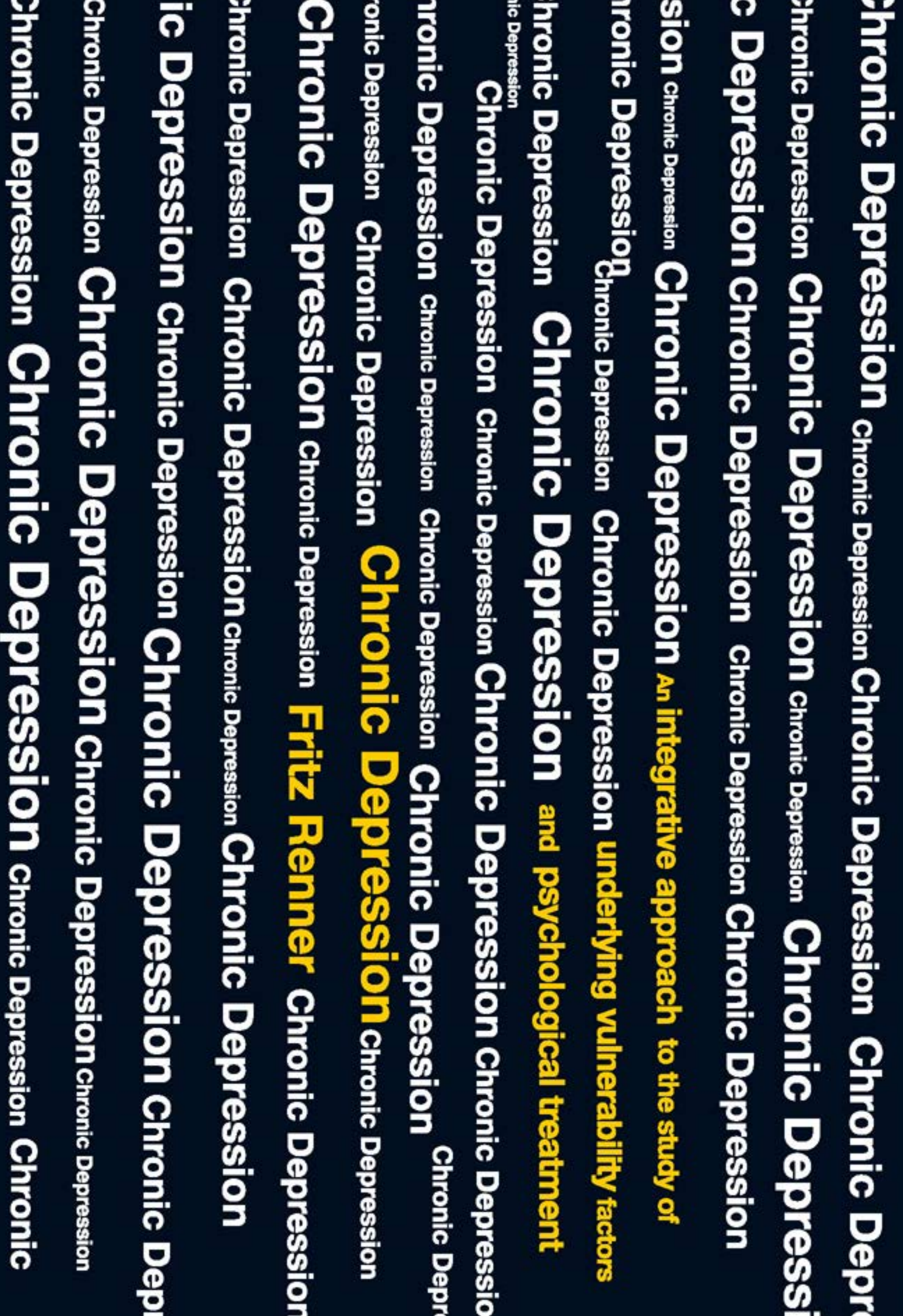

